IS AN ALIBI A PAPER SHIELD?

\title{
AN INVESTIGATION OF THE FACTORS THAT INFLUENCE ALIBI CREDIBILITY JUDGMENTS
}

\author{
By \\ Sara Cowan \\ Master of Arts, Ryerson University, Toronto, ON, 2012 \\ Bachelor of Arts (Honours), University of Victoria, Victoria, BC, 2009
}

\author{
A dissertation \\ Presented to Ryerson University \\ in partial fulfillment of the \\ requirements for the degree of \\ Doctor of Philosophy \\ in the Program of \\ Psychology
}

Toronto, Ontario, Canada, 2016

CSara Cowan 2016 


\section{AUTHOR'S DECLARATION FOR ELECTRONIC SUBMISSION OF A DISSERTATION}

I hereby declare that I am the sole author of this dissertation. This is a true copy of the dissertation, including any required final revisions, as accepted by my examiners.

I authorize Ryerson University to lend this dissertation to other institutions or individuals for the purpose of scholarly research

I further authorize Ryerson University to reproduce this dissertation by photocopying or by other means, in total or in part, at the request of other institutions or individuals for the purpose of scholarly research.

I understand that my dissertation may be made electronically available to the public. 


\title{
IS AN ALIBI A PAPER SHIELD?
}

\section{AN INVESTIGATION OF THE FACTORS THAT INFLUENCE ALIBI CREDIBILITY JUDGMENTS}

Sara Cowan

Doctor of Philosophy, 2016

Psychology

Ryerson University

\begin{abstract}
Alibis are a potentially powerful piece of evidence for innocence, but examination of criminal cases suggests that honestly offered alibis may fail to prevent wrongful convictions. Currently, little is known regarding how evaluators judge the credibility of alibis. Three studies investigated the effect of alibi moral desirability, suspect race (White/Indigenous Canadian), alibi evidence strength, and Authoritarianism on participants' legal judgments. Participants read a fictitious police file (Experiment 1: $N=300$; Experiment 2: $N=286$ ) or newspaper article (Experiment 3: $N=235$ ) and rated a male suspect's/defendant's statement honesty, alibi accuracy, and the likelihood of his guilt, among other dependent measures, then completed the AuthoritarianismConservatism-Traditionalism scale (ACT; Duckitt et al., 2010) and, in Experiment 3, the Revised Religious Life Inventory (Hills et al., 2005). In Experiments 1 and 2, participants were asked to sign a petition supporting the suspect. Results indicated that providing an alibi can be beneficial or detrimental to the suspect, depending on contextual factors and the narrative itself. In Experiments 1 and 2, alibi moral desirability affected participants' responses, though different patterns emerged at Ryerson and at Iowa State, and moral desirability influenced judgments primarily for the Indigenous suspect. Consistent with Olson and Wells' (2004) taxonomy,
\end{abstract}


Experiment 1 showed that the strength of the physical evidence supporting an alibi is a primary determinant of judgments of its credibility. In Experiment 3, participants provided less favourable ratings for the Indigenous defendant than the White defendant, particularly when they already had more negative general feelings about Indigenous people, though this was not found in Experiment 2. More participants signed the petition when the alibi was morally desirable at Iowa State, and for the Indigenous suspect. Across all studies, higher scores on the ACT's Authoritarianism subscale were associated with responses that were less favourable for the suspect/defendant, and many participants did not accurately define the term "alibi." Understanding the complexities of decision-making in this context will help us better understand why some (honest) alibis are rejected, and how stereotypes and assumptions regarding the alibi provider may lead to bias in the investigation and adjudication of criminal cases. 


\section{Acknowledgements}

Probably the best description of how it feels to write a dissertation can be found in George RR Martin's acknowledgements of his book, A Dance with Dragons, when he wrote "this one was three bitches and a bastard" (Martin, 2011, p. 1017). This process has been the most intellectually, physically, and emotionally demanding experience of my life (so far). There have been sweat and tears. Not too much blood, fortunately. I owe an enormous debt of gratitude to the many people who supported me throughout. Without them, none of this would have been possible.

I have been fortunate in so many ways to have Dr. Tara Burke as my supervisor and mentor. She has pushed me to grow by asking the tough questions and has given me the freedom to chart my own path through the PhD. She has always had my best interests at heart. Her patience, insight, support, and humour were invaluable to the process. I also could not have had a better supervisory committee. Dr. Becky Choma managed to push me as hard as possible to achieve my full potential and was incredibly supportive and kind while doing it. Not even typos escaped her watchful gaze. Dr. Ben Dyson brought his expertise in cognitive psychology to a social psychology dissertation — from another continent no less! — and, in doing so, made the work much stronger than it otherwise would have been. I am also thankful that Dr. Joanna Pozzulo and Dr. Graham Hudson served on my examining committee and made the defence itself a positive learning experience.

There are many people without whom the research presented here would never have been completed. My sincerest thanks to Carson Pun for creating three fake petitions and never making me feel stupid for asking stupid questions. Thanks to Emily Lockhart for making the petition logo, and offering to edit it when we realized that the original version was inadvertently hilarious. Thanks as well to Patryce Bowling, who let me bribe her with food to do untold hours of coding on all three studies. Thanks to Lisa Vuong for answering a million questions, keeping me from forgetting to submit various forms, and being generally awesome. Thanks to Dr. Chris Meissner for allowing me to collect data at Iowa State (even if the between-sample differences did end up being the bane of my existence for several months). Thanks to my dear friends Jen Rouse and Jessica Lockhart for the weekend work dates that did not allow me to procrastinate. If it wasn't for you both, I probably wouldn't have finished on time. Seriously. And finally, thanks to the baristas who kept me plied with sweet, sweet caffeine throughout.

My colleagues and friends in the Psychology and Law lab provided immeasurable support throughout my graduate years. Thanks for giving advice, letting me talk out loud even when I wasn't making sense, keeping me from getting too hangry (seriously, though, sometimes I was grumpy because things were legitimately annoying!), being ridiculously fun conference travel buddies, and giving amazing feedback. Love and gratitude to: Stéphanie Marion, Sami ElSibaey, Lesley Zannella, and Stuart Freedman.

Thanks as well to friends who let me complain, celebrated the victories, commiserated with me on the setbacks, forced me to actually leave the house and have fun occasionally, kept me moderately sane, helped with any number of catastrophes, and put up with me being a hermit for most of the past year. There are too many of you to count, but special recognition is due to 
Ash Ward, Nicole Sugden, Jen Rouse, Amy Brown-Bowers, Amy Usher, Jessica Lockhart, Julia Boyle, Patryce Bowling, Nicole Cormier, Bonnie Armstrong, Adam Bailey, Sydney Fletcher, Ben Alexander, and the members of the Corktown Chamber Orchestra. You all kept me from quitting on account of how embarrassing it would have been to have to tell everyone.

I also owe a weird debt of gratitude to Barenaked Ladies (yes, the band). It's a long story.

Last, but never least, thanks so much to my family. To my parents, Sherry Lepage and John Cowan, who always believed in me. Thank you for raising me in a home filled with books, music, and intellectual dinner table conversation. This whole $\mathrm{PhD}$ thing is largely your fault. See what happens when you encourage people! Thanks to my brother, Devin Cowan, for the jokes and the laughs; my aunt, Yvette Lepage, for being almost irritatingly wise; my grandmère, Cecile Lepage, for always being interested in what I was up to; to my grandparents, Roy and Irish (Isabella) Cowan, for being a loving force throughout my life, even though neither of them are here to celebrate with me today; and to my cat, Violet, who gives me all the snuggles. I love you all. 


\section{Dedication}

This dissertation is dedicated to anyone brave or stupid enough to take on a $\mathrm{PhD}$.

But especially to my dear friend Chad Yacobucci, whose PhD plans have been disrupted by the cruel, merciless havoc of cancer.

I hope you win both battles. 


\section{Table of Contents}

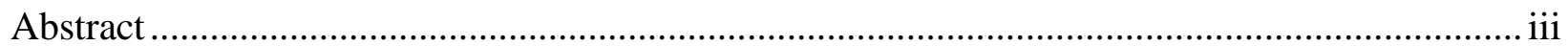

List of Tables ........................................................................................................... xiv

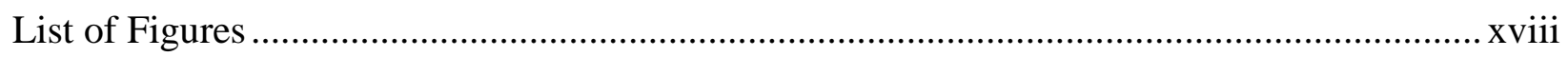

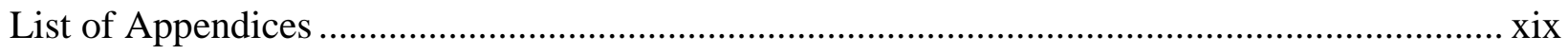

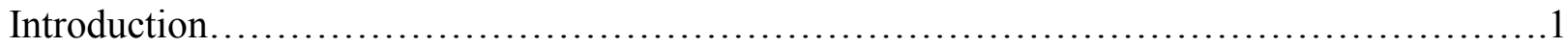

The Problem of Wrongful Conviction (or 11,600 Years of Solitude) .................................... 3

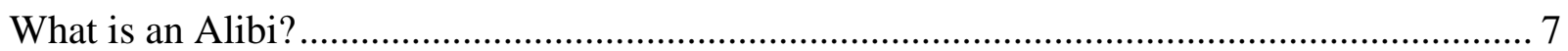

The Practical Utility of Alibis for Protecting Innocent Suspects and Defendants ................... 9

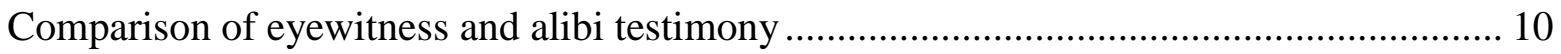

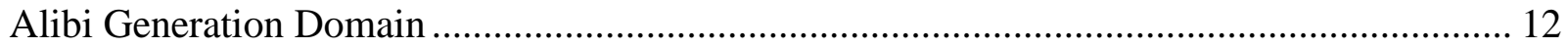

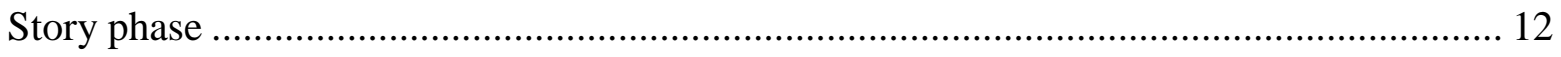

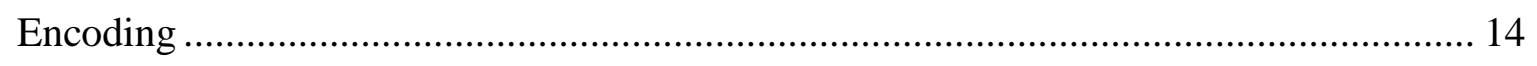

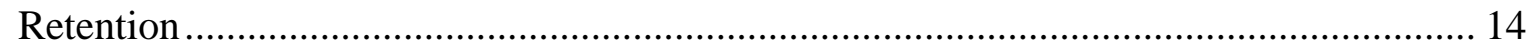

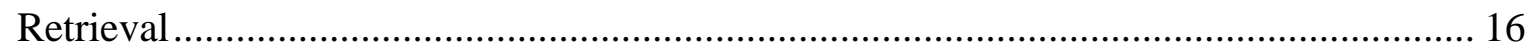

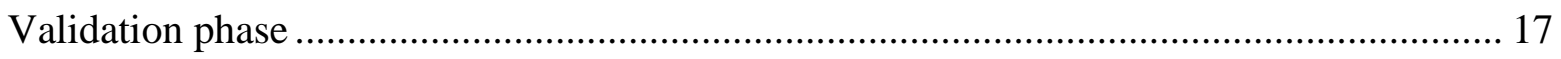

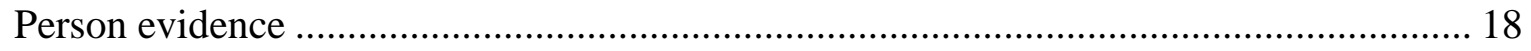

What evidence is typically available to defendants? ............................................... 20

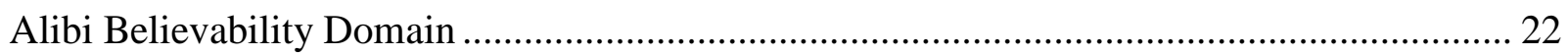

General skepticism and the alibi generation effect................................................. 22

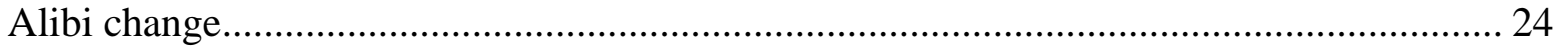

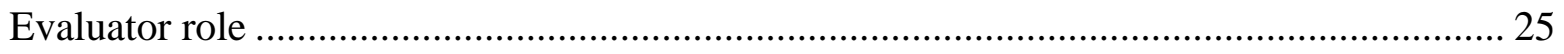

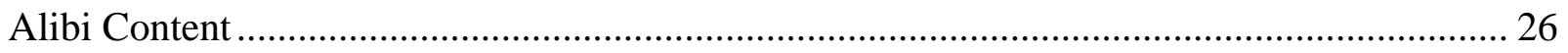

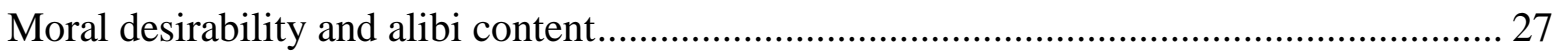

Attributions, implicit personality theories, alibis, and legal outcomes ........................... 28

Morally undesirable alibis and the assumption of honesty ......................................... 32

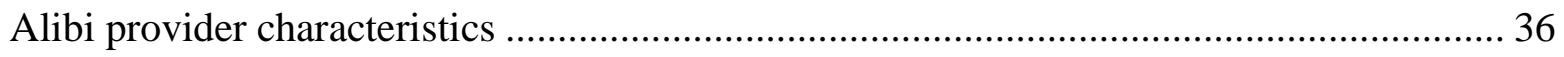




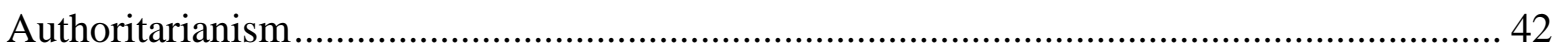

Summary of Literature Review …………………………............................................. 43

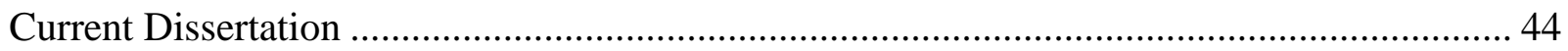

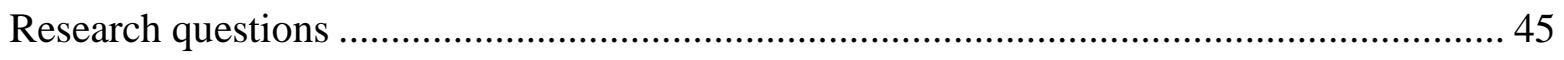

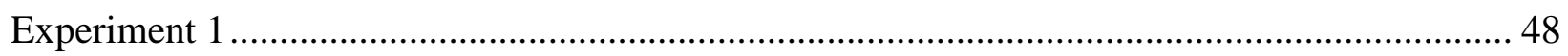

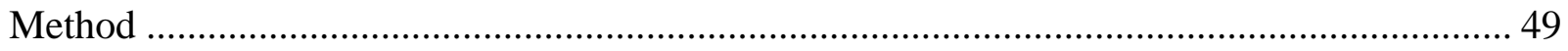

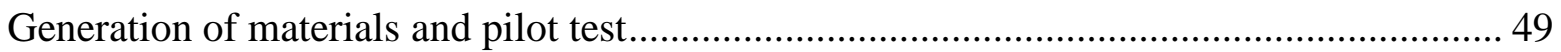

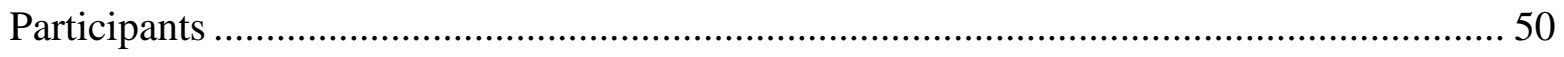

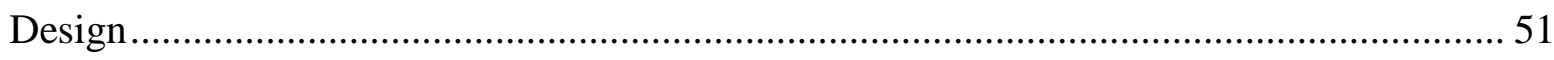

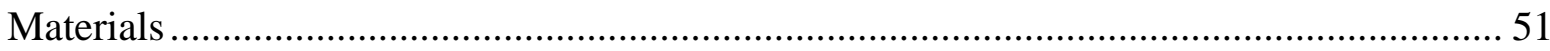

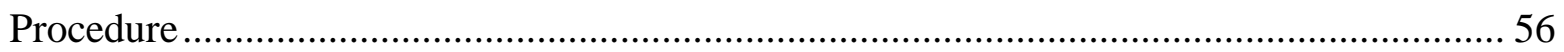

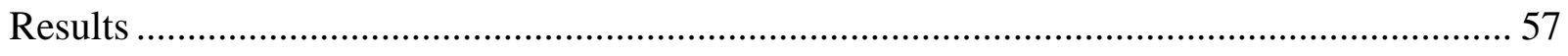

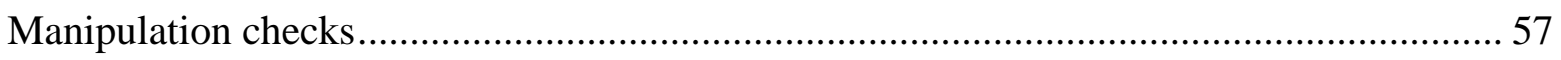

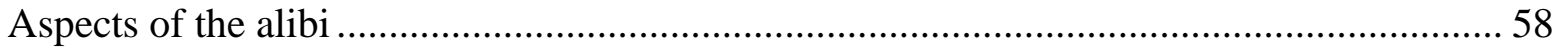

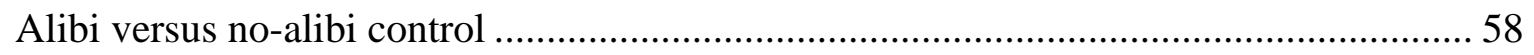

Comparisons of ratings of the different alibis and the no-alibi control ............................. 61

The effect of alibi content, corroborative evidence, and sample location .......................... 64

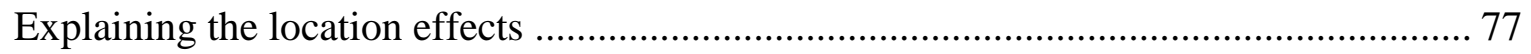

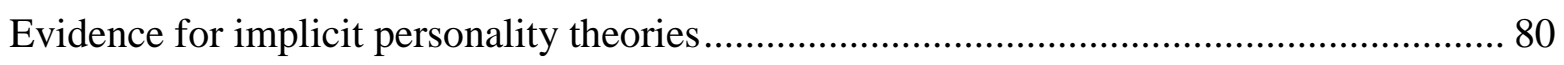

Do perceptions of alibi moral desirability affect legal judgments through liking of the

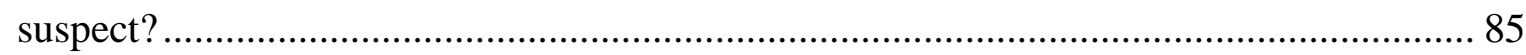

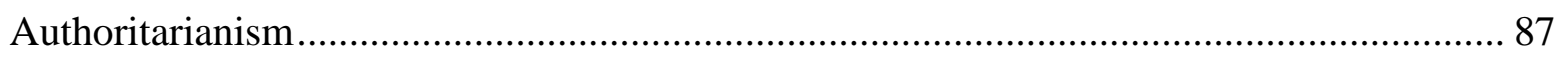

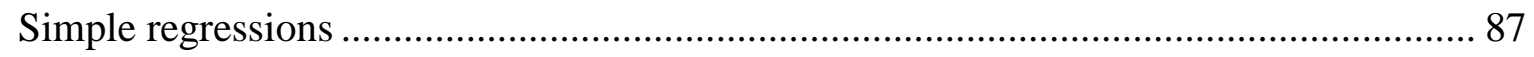

Mediation effects of alibi moral desirability............................................................... 89

Qualitative analysis of participants' definitions of an "alibi." .............................................. 93

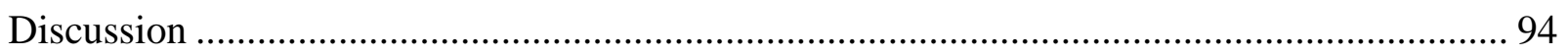

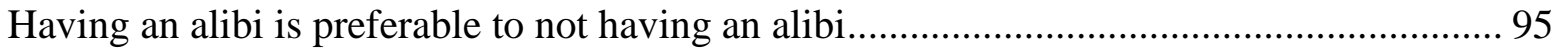

The effect of moral desirability on legal judgments and its interaction with evidence strength 
The effect of alibi narratives on ratings of similarity, liking, and the overall evaluation of the suspect's character, and mediational effects of the former ........................................ 98

Differences between the Ryerson University and Iowa State University samples .............. 98

Authoritarianism and the mediational effects of perceived alibi moral desirability .......... 102

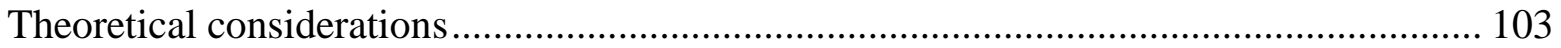

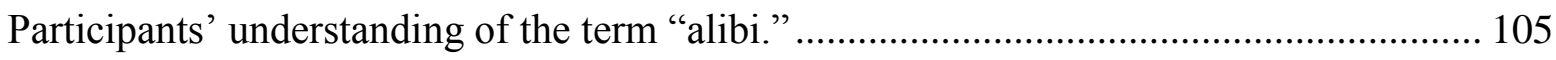

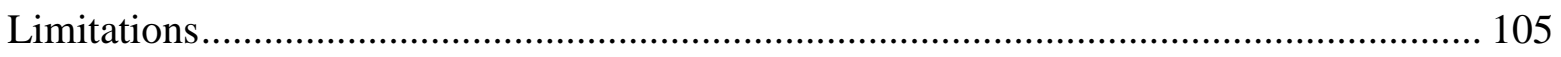

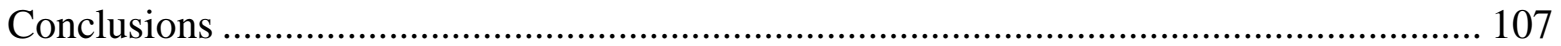

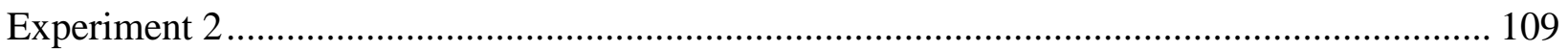

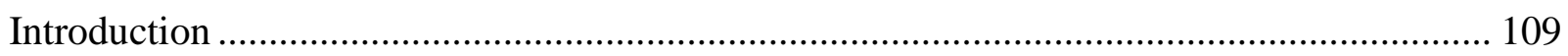

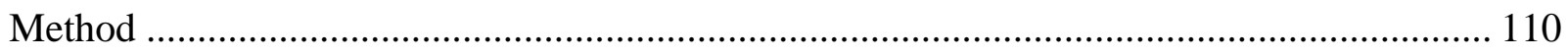

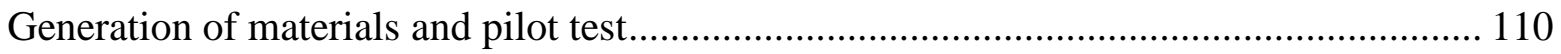

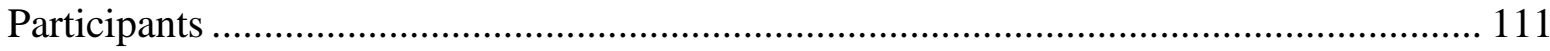

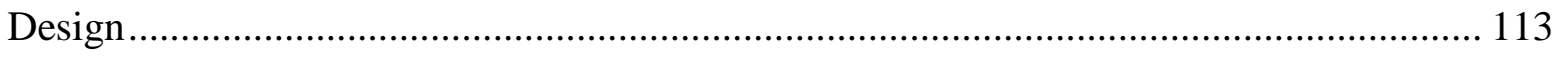

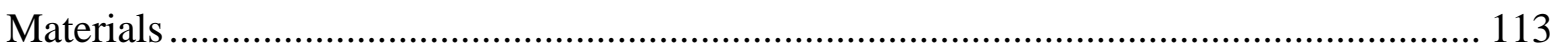

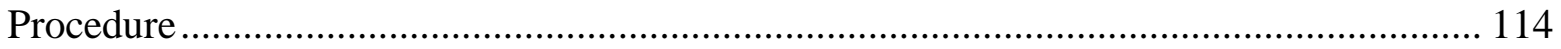

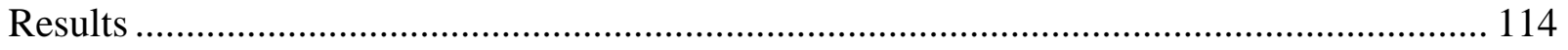

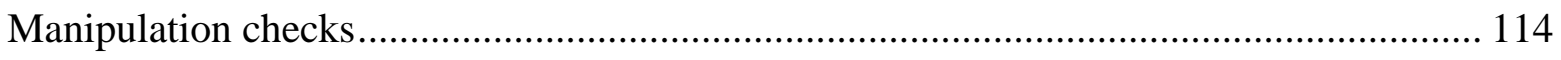

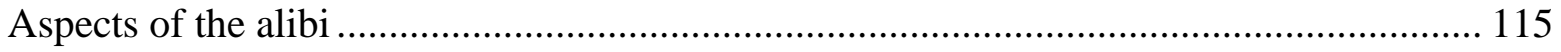

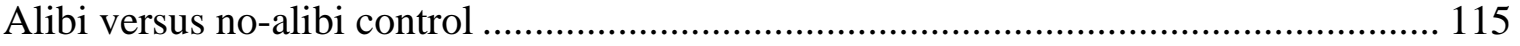

The effect of alibi content, alibi provider race, and crime location ............................. 117

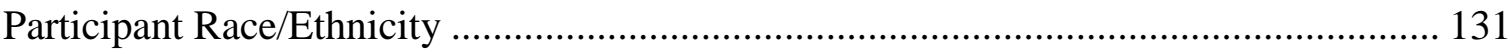

The influence of participants' general feelings about First Nations and White people on

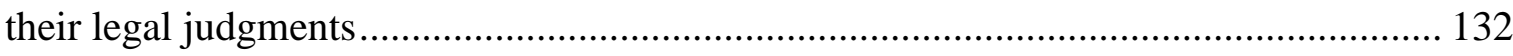

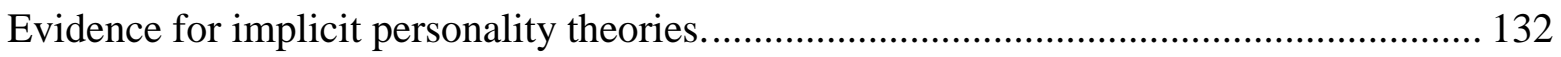

Do perceptions of alibi moral desirability affect legal judgments through liking of the

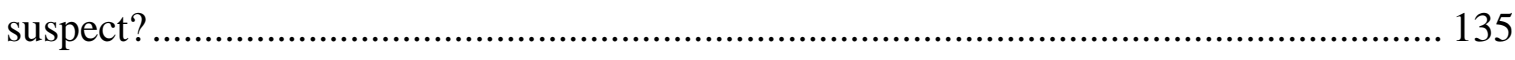

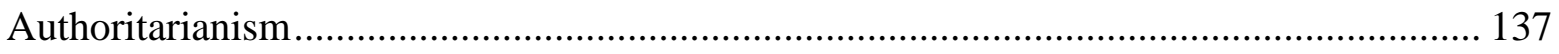

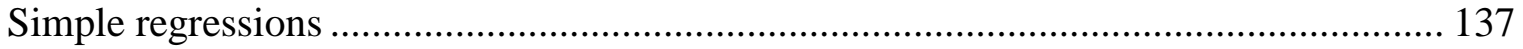

Mediation effects of alibi moral desirability .......................................................... 137 
Qualitative analysis of participants' definitions of an "alibi" 140

Discussion

Failing to generate an alibi may be preferable to providing one.....

The effect of moral desirability on legal judgments and its interaction with suspect race . 146

Crime location and in-group bias

Participant race/ethnicity is unrelated to legal judgments

Participants' general feelings about First Nations and White people are unrelated to their

legal judgments

Judgments of similarity, liking, and the overall evaluation of the suspect's character ....... 152

Authoritarianism and the mediational effects of perceived alibi moral desirability .......... 152

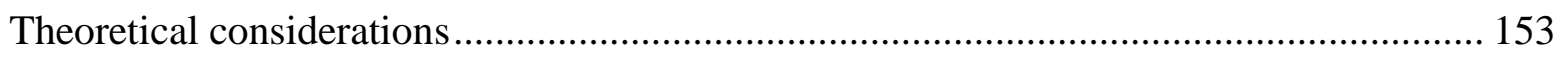

Participants' understanding of the term "alibi." ........................................................... 154

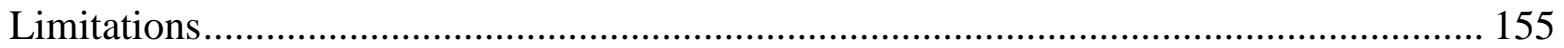

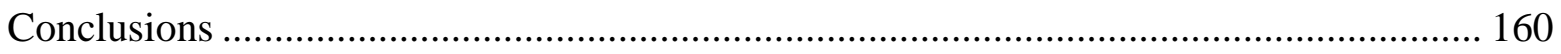

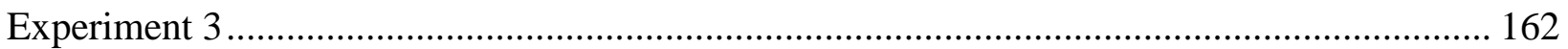

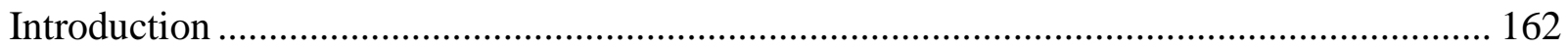

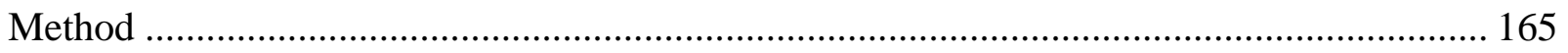

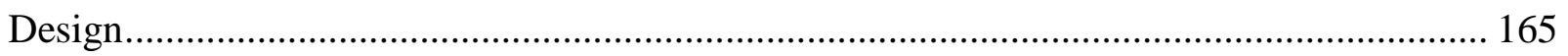

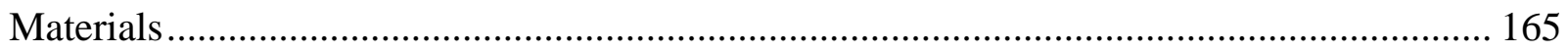

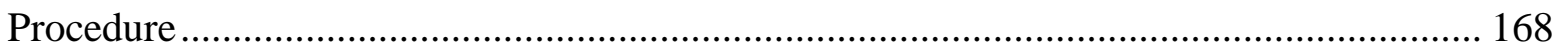

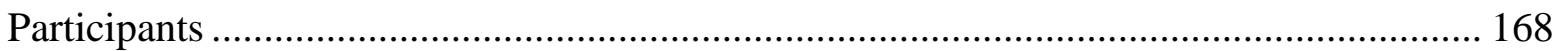

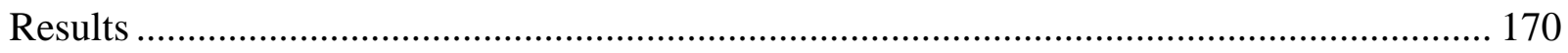

The effect of defendant race, crime location, and sample location on legal judgments..... 170

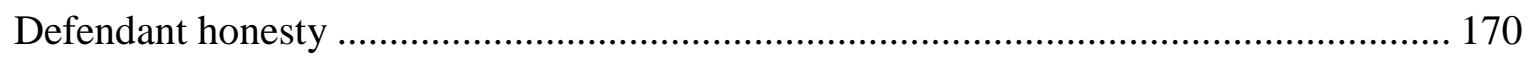

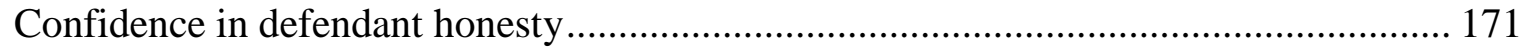

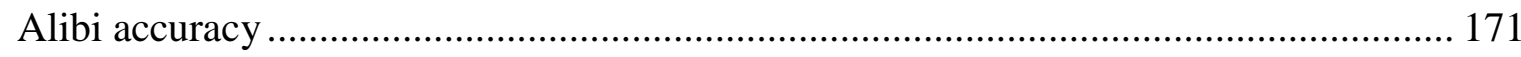

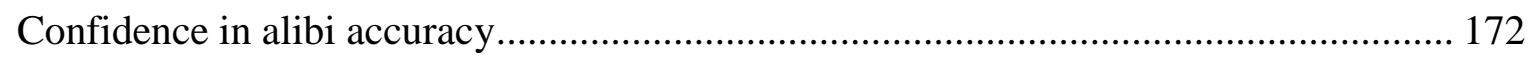

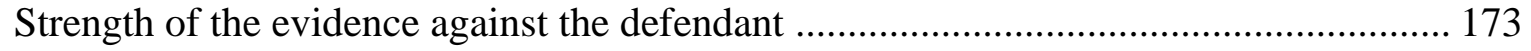

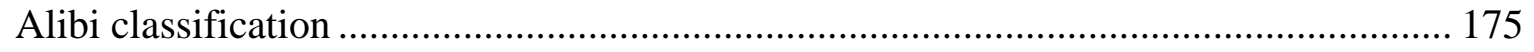




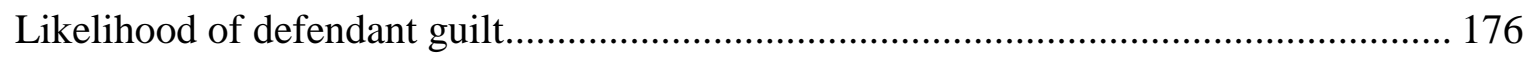

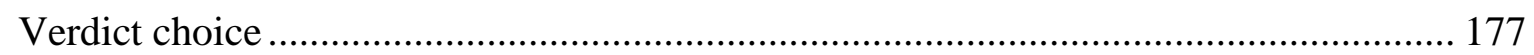

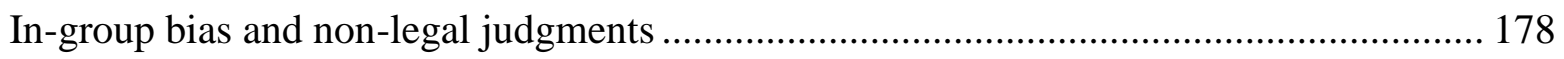

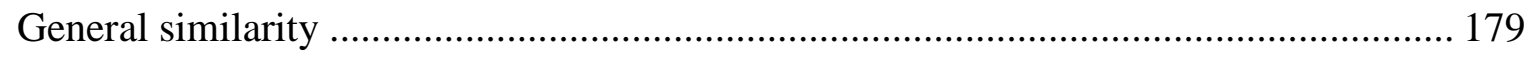

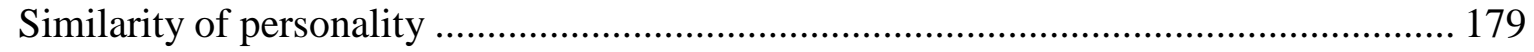

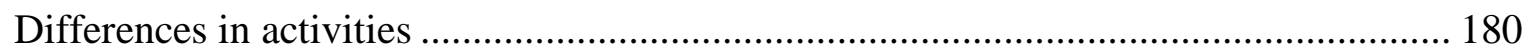

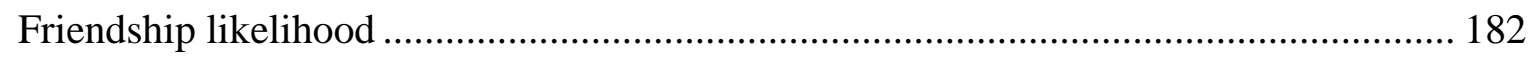

Is the defendant a good or bad person?................................................................... 182

Summary of effects for non-legal dependent variables ................................................ 183

The influence of participants' general feelings about First Nations and White people on their

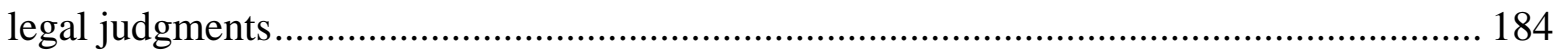

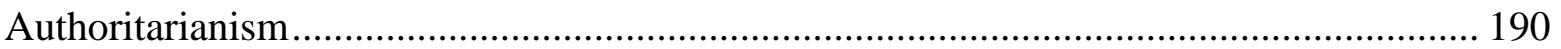

Religious orientation .............................................................................................. 192

Does level of endorsement of particular religious orientations predict legal judgments? 193

Do high-responders and low-responders on the RLI-R differ in their legal judgments? 195

Qualitative analysis of participants' definitions of an "alibi." .............................................. 196

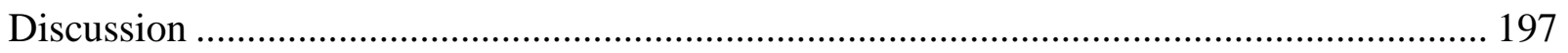

The effects of defendant race, crime location, and sample location on legal judgments .... 197

Participants' general feelings about White and First Nations people.................................. 198

The effect of defendant race, crime location, and sample location on non-legal judgments

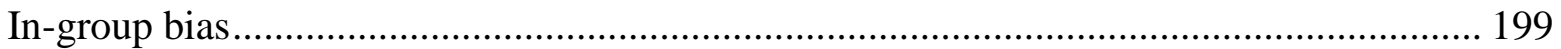

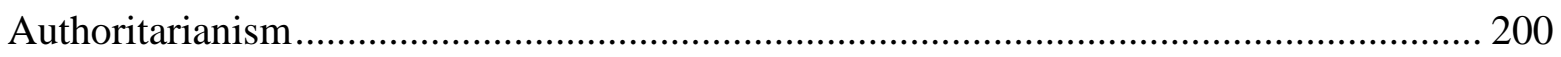

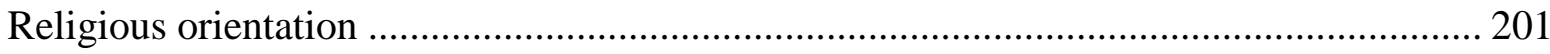

Participants' definition of the term "alibi." .................................................................. 202

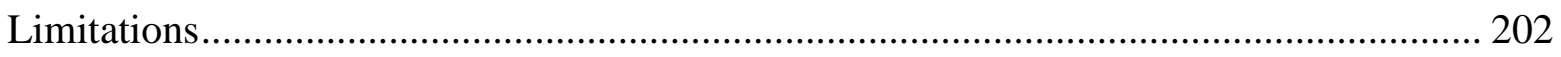

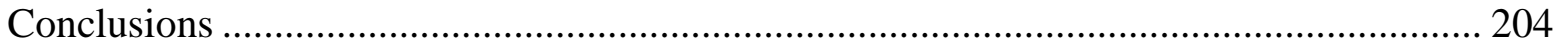

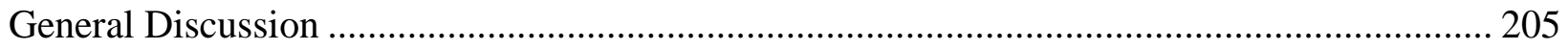

Providing an Alibi Can Be Beneficial or Detrimental for Criminal Suspects......................... 206

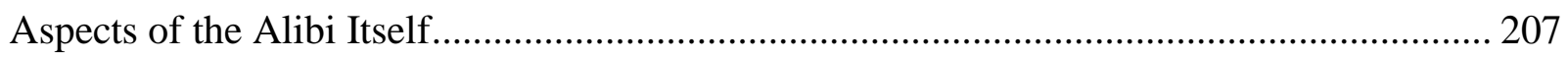




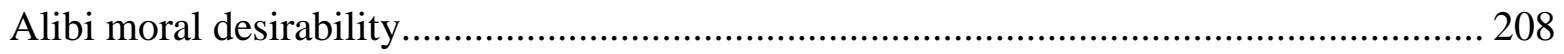

The best defense is strong physical evidence ............................................................... 212

Alibi Provider Characteristics - Suspect/Defendant Race ............................................. 214

Alibi Evaluator Characteristics .............................................................................. 216

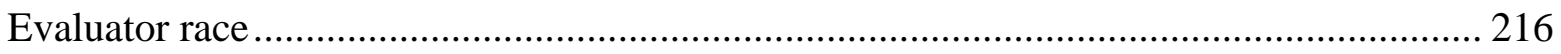

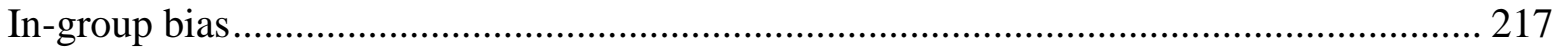

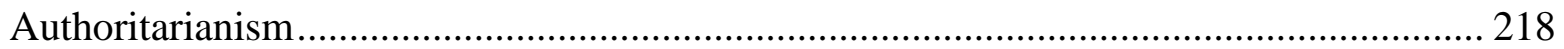

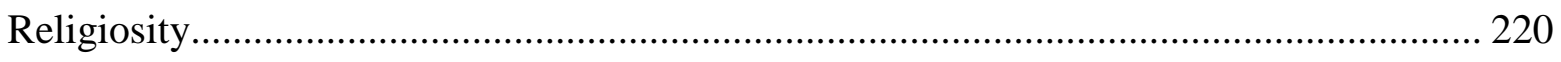

Misunderstandings About the Term “Alibi” Abound ....................................................... 221

Implications for Research and Practice ................................................................ 222

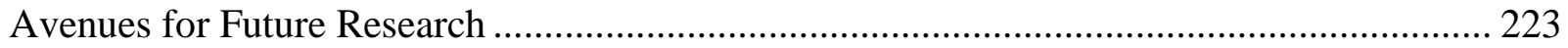

General Conclusions ....................................................................................... 226

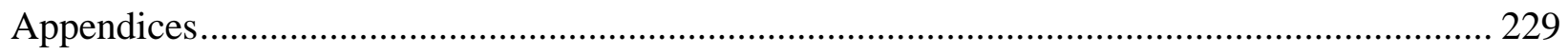

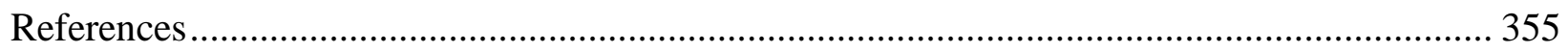




\section{List of Tables}

Table 1: Participant demographic information.......................................... 52

Table 2: Descriptive statistics for comparison of alibi vs. no-alibi control conditions by

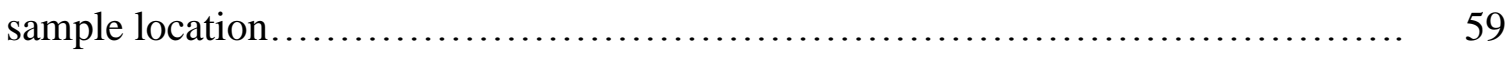

Table 3: Rates of petition-signing at Iowa State and Ryerson $\ldots \ldots \ldots \ldots \ldots \ldots \ldots \ldots \ldots \ldots \ldots$

Table 4: Means and standard deviations for alibi and control conditions $\ldots \ldots \ldots \ldots \ldots \ldots \ldots .62$

Table 5: ANOVA results for participants' ratings of suspect honesty $\ldots \ldots \ldots \ldots \ldots \ldots \ldots \ldots .65$

Table 6: ANOVA results for participants' confidence in their ratings of suspect honesty.... 66

Table 7: ANOVA results for participants' ratings of alibi accuracy $\ldots \ldots \ldots \ldots \ldots \ldots \ldots \ldots \ldots .66$

Table 8: ANOVA results for participants' confidence in their ratings of alibi accuracy...... 67

Table 9: ANOVA results for participants' ratings of the strength of the evidence against

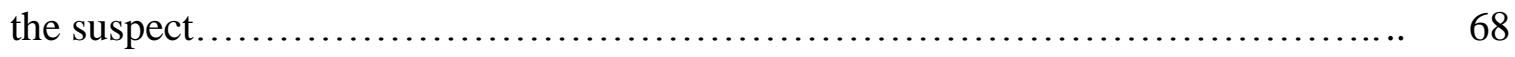

Table 10: Participants' classifications of the suspect's alibi as percentages............... 69

Table 11: ANOVA results for participants' ratings of the likelihood of suspect guilt....... 71

Table 12: ANOVA results for participants' verdict choice.......................... 74

Table 13: ANOVA results for participants' willingness to sign the petition............... 77

Table 14: Linear regression models using ratings of liking and similarity to predict legal judgments..........................................................

Table 15: Binary logistic regression models using ratings of liking and similarity to predict verdict choice and petition signing.....

Table 16: Descriptive statistics for similarity, liking, and suspect's overall character by

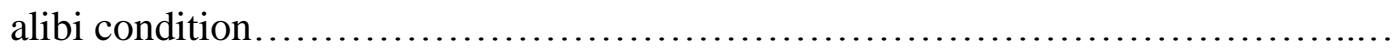

Table 17: Pairwise comparison of different levels of alibi moral desirability on similarity

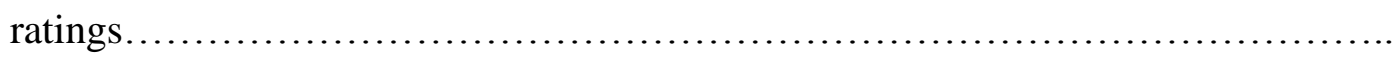

Table 18: Results of mediation analyses 
Table 19: Statistical results for linear regression models using ACT total and subscale scores to predict participants' responses on dependent measures....................

Table 20: Statistical results for binary logistic regression models using ACT total and subscale scores to predict participants' verdict choice and petition signing decision.

Table 21: Results of mediation analyses........................................ 91

Table 22: Participant race/ethnicity

Table 23: t-test results comparing ratings of participants who were shown an alibi and participants who were not shown an alibi.

Table 24: ANOVA results for participants' ratings of suspect honesty....

Table 25: Pairwise comparisons of participants' ratings of statement honesty under different alibi conditions, when the suspect is First Nations.

Table 26: ANOVA results for participants' confidence in their ratings of suspect honesty... 120

Table 27: ANOVA results for participants' ratings of alibi accuracy....

Table 28: ANOVA results for participants' confidence in their ratings of alibi accuracy......

Table 29: ANOVA results for participants' ratings of the strength of the evidence against the suspect.

Table 30: Pairwise comparisons of participants' ratings of the strength of the evidence against the suspect under different alibi conditions

Table 31: ANOVA results for participants' ratings of the likelihood that the suspect is guilty.

Table 32: Pairwise comparison of guilty verdict rates for different alibi conditions ....

Table 33: Descriptive statistics for participants' ratings of similarity, liking, and whether the suspect is a good or bad person, separated by Alibi Content group.

Table 34: Pairwise comparison of different levels of alibi moral desirability on similarity ratings.

Table 35: Results of mediation analyses. 
Table 36: Statistical results for linear regression models using ACT total and subscale scores to predict participants' responses on dependent measures.................. 138

Table 37: Statistical results for binary logistic regression models using ACT total and subscale scores to predict participants' verdict choice and petition signing decision. 139

Table 38: Results of mediation analyses........................................... 141

Table 39: Participant demographic information...................................... 169

Table 40: ANOVA results for participants' ratings of the defendant's honesty............. 171

Table 41: ANOVA results for participants' confidence in their ratings of the defendant's

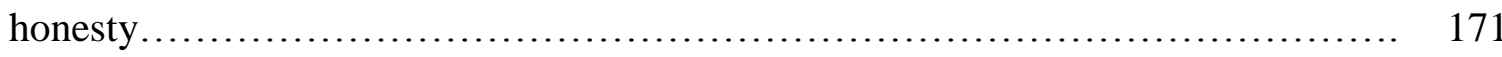

Table 42: ANOVA results for participants' ratings of alibi accuracy.................... 172

Table 43: ANOVA results for participants' confidence in their ratings of alibi accuracy..... 173

Table 44: ANOVA results for participants' ratings of the strength of the evidence against

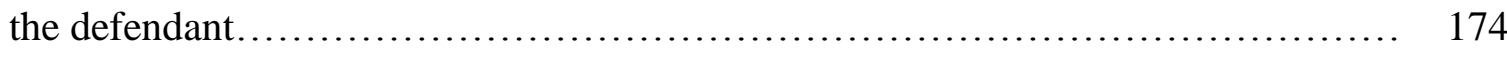

Table 45: Participants' classifications of the defendant's alibi as percentages.............. 175

Table 46: ANOVA results for participants' ratings of the likelihood that the defendant is

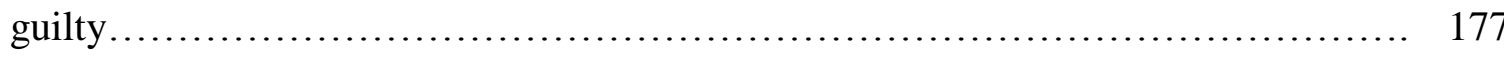

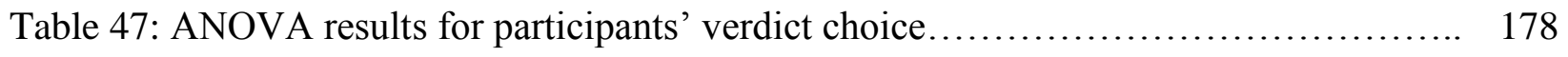

Table 48: ANOVA results for participants' ratings of how similar they are in general to the

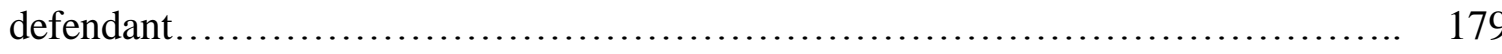

Table 49: ANOVA results for participants' ratings of how similar their personalities are to the defendant............................................................. 180

Table 50: ANOVA results for participants' ratings of how different the activities they

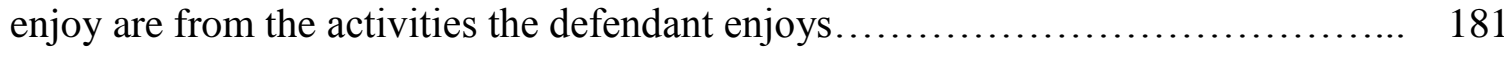

Table 51: ANOVA results for participants' ratings of the likelihood of them becoming

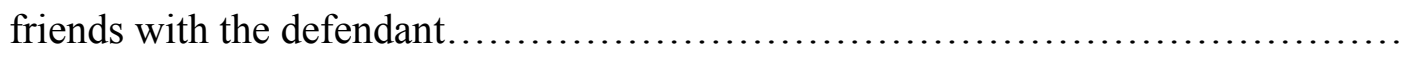

Table 52: ANOVA results for participants' ratings of whether the defendant is a good or 
bad person overall.

Table 53: Simple and multiple linear regression models using participants' general feelings about White and/or First Nations people to predict their judgments about White or First Nations suspects

Table 54: Simple and multiple binary logistic regression models using participants' general feelings about White and/or First Nations people to predict their judgments about White or First Nations suspects

Table 55: Statistical results for linear regression models using ACT total and subscale scores to predict participants' responses on dependent measures

Table 56: Statistical results for binary logistic regression models using ACT total and subscale scores to predict participants' verdict choice and petition signing decision.

Table 57: Statistical results for linear regression models using RLI-R subscale scores to predict participants' responses on dependent measures

Table 58: Statistical results for binary logistic regression model using RLI-R subscale

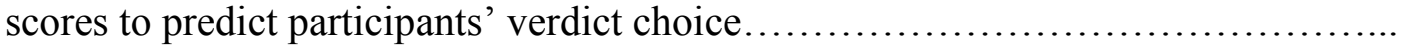

Table 59: Comparison of legal judgments between Religious and Non-Religious participants 


\section{List of Figures}

Figure 1: Mean ratings of statement honesty for different levels of alibi corroboration at Ryerson and Iowa State.

Figure 2: Mean ratings of the strength of the evidence against the suspect for different alibis, at Ryerson and Iowa State

Figure 3: Mean ratings of the likelihood of suspect guilt for different alibi narratives and different levels of corroboration, at Iowa State and at Ryerson.

Figure 4: Percent of participants who signed the petition for different alibi narratives and different levels of corroboration, at Iowa State and at Ryerson

Figure 5: Mean ratings of suspect honesty for White and First Nations suspects for different alibi narratives

Figure 6: Mean ratings of suspect honesty for White and First Nations suspects for different alibi narratives

Figure 7: Mean self-reported confidence of participants' ratings of suspect honesty for White and First Nations suspects for different alibi narratives....

Figure 8: Participants' ratings of the strength of the evidence against the suspect for White and First Nations suspects who offered different alibis.

Figure 9: Participants' mean self-reported confidence in their ratings of the accuracy of the defendant's alibi

Figure 10: Participants' mean ratings of the strength of the evidence against the suspect.....

Figure 11: Participants' mean ratings of the likelihood that the defendant is guilty

Figure 12: Participants' ratings of how similar they are in terms of personality to the defendant as a function of Crime Location and Defendant Race.

Figure 13: Comparison of Canadian and American participants' ratings of the dissimilarity of the activities that they and the First Nations or White defendant enjoy

Figure 14: Correlation between participants' general feelings about First Nations and White people. 


\section{List of Appendices}

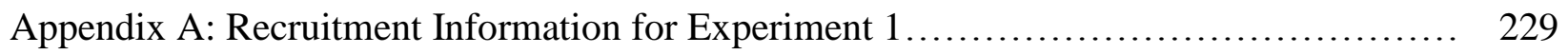

Appendix B: Consent Forms for Experiment 1 ................................... 232

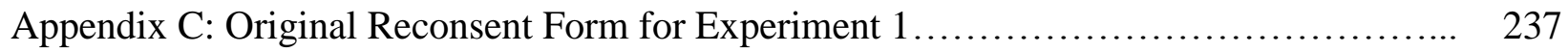

Appendix D: Revised Reconsent Form for Experiment 1 (September, 2015)............... 238

Appendix E: Experiment 1 Materials.............................................. 239

Appendix F: Experiment 1 Questionnaire ........................................ 250

Appendix G: Authoritarianism-Conservatism-Traditionalism Scale (Duckitt et al., 2010)_.. 254

Appendix H: Petition Website for Experiment 1................................... 255

Appendix I: Experiment 1 Manipulation Checks..................................... 256

Appendix J: Exploring Location Effects in Experiment 1............................ 259

Appendix K: Psychometric Analysis of the ACT for Experiment 1..................... 266

Appendix L: Codebook for Qualitative Data; All Experiments.......................... 269

Appendix M: Inferential Tests Based on Qualitative Responses for Experiment 1......... 270

Appendix N: Experiment 1 Debrief Forms........................................ 271

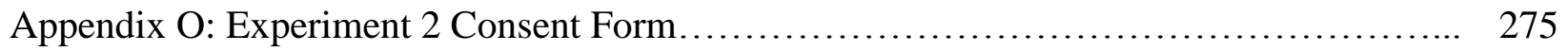

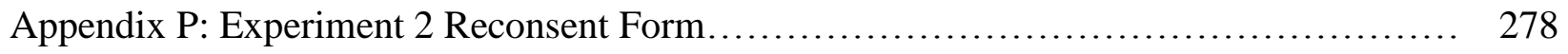

Appendix Q: Experiment 2 Sona Recruitment Information............................. 279

Appendix R: Experiment 2 Case Materials .......................................... 280

Appendix S: Experiment 2 Questionnaire ........................................ 304

Appendix T: Experiment 2 Petition Websites....................................... 309

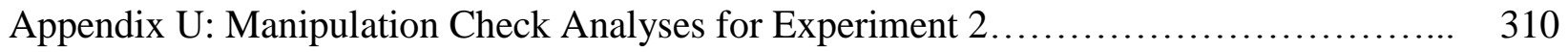

Appendix V: Analyses for Participant Race/Ethnicity in Experiment 2................. 313 
Appendix W: Analyses of Participants' General Feelings about White and First Nations People in Experiment 2 ........................................... 316

Appendix X: Psychometric Analyses of the ACT for Experiment 2................... 323

Appendix Y: Inferential Tests Based on Qualitative Responses for Experiment 2........ 326

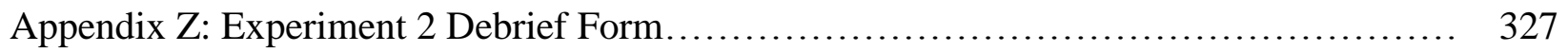

Appendix AA: Experiment 3 Recruitment Information............................. 329

Appendix BB: Experiment 3 Consent Forms ................................. 330

Appendix CC: Experiment 3 Fictitious Newspaper Articles........................ 333

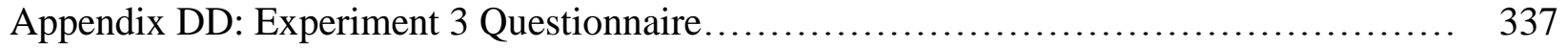

Appendix EE: Revised Religious Life Inventory (RLI-R; Hills et al., 2005)............. 344

Appendix FF: Experiment 3 Non-Significant Results for Non-Legal Judgments.......... 345

Appendix GG: Psychometric Analyses of the ACT and RLI-R for Experiment 3......... 348

Appendix HH: Inferential Tests Based on Qualitative Responses in Experiment 3 ........ 352

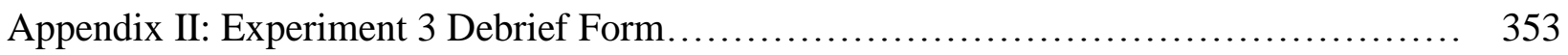


"Juries frequently convict defendants despite impressive alibi testimony or other persuasive evidence" (Saunders, 1984, p. 190)

On the afternoon of July 29, 1985, Penny Ann Beerntsen was jogging along the shore of Lake Michigan near Two Rivers, Wisconsin. At 3:50pm, a disheveled-looking man that she had seen earlier grabbed her from behind, dragged her into the bushes, and violently sexually assaulted her. He then choked her until she lost consciousness and then he ran off at approximately 4:05pm.

Until 3:30pm that same day, Steven Avery was pouring concrete at his parents' salvage yard, 12 miles (19.3km) from the scene of the assault. He, his wife, and five children left the salvage yard at 4:30pm and drove to Green Bay, where they obtained food at a Burger King drive-thru, got a car wash, and purchased paint. The time on the receipt for the paint purchase at Shopko, 36 miles $(57.9 \mathrm{~km})$ away from the crime scene, was $5: 13 \mathrm{pm}$. Despite the absence of any evidence linking him to the crime, police suspected that Avery had committed the assault. He had previous convictions in 1981 for burglary and animal cruelty. Additionally, earlier in 1985, he ran the wife of a police officer off the road and pointed a rifle at her; he was later convicted of endangering safety and felony gun possession related to that incident and sentenced to six years in prison. Avery was arrested only hours after the assault on Ms. Beerntsen occurred. Beerntsen identified Avery in a photo and a live lineup that are now considered to have employed extremely biased procedures. Avery was the only individual included in both photo lineups, and it is alleged that she was shown a mugshot of Avery as well as a sketch that may have been drawn from the mugshot prior to being shown the lineups. Both of these events are known to increase false identifications (e.g., Steblay, Tix, \& Benson, 2013). It is also alleged that police 
encouraged Beerntsen to identify Avery during the lineup procedures.

A second individual, Gregory Allen, was not thoroughly investigated by police, despite an extensive criminal record spanning four US States, being a suspect in a long list of other crimes, and having been convicted of an offense similar to the assault on Beerntsen two years previously. Manitowoc County police were aware of his record and had received authorization for surveillance on him after he became a suspect in an attempted rape two weeks before the assault on Beerntsen. On the date of Beerntsen's assault, they only confirmed his whereabouts once. Despite Allen being a viable suspect, police never showed Beerntsen a photo of him.

At Avery's trial in December, 1985, the defense presented sixteen witnesses to support the alibi, including a store clerk from the Green Bay Shopko who testified that Avery had purchased a gallon of paint from her approximately an hour after the assault occurred. Avery also provided a receipt for the paint. Despite this evidence, Stephen Avery was convicted of sexual assault, false imprisonment, and attempted murder on the basis of Beerntsen's identification, and was sentenced to 32 years in prison. On September 11, 2003, after spending 17.5 years in prison, Avery was exonerated based on the analysis of DNA evidence that showed that Gregory Allen was the true perpetrator. Gregory Allen had gone on to commit several more sex crimes after 1985, and was sentenced to a 60 -year prison term for a brutal rape committed in Green Bay in 1995. (Cruz, 2015; Findley, 2011; Kertscher, 2003; Literary Ramblings, 2016; National Registry of Exonerations, 2012; Northwestern Law, n.d.; Sherrer, 2012; France, 2016; The Forgiveness Project, 2010; The Innocence Project, n.d.a; True Crime XL, 2012).

A key question in this case is why Steven Avery's alibi failed to protect him from wrongful prosecution and conviction, particularly when the evidence supporting his statement was quite strong. The fact that he was almost immediately arrested and held in prison for 8 days 
without outside contact virtually eliminates the possibility that he fabricated the alibi witness evidence (Sherrer, 2012). Currently, the process by which evaluators assess the credibility of alibis is not well understood. The research literature indicates that the quality of evidence supporting an alibi (e.g., Olson \& Wells, 2004), characteristics of the provider (e.g., Sargent \& Bradfield, 2004), the context in which the judgment is made (Sommers \& Douglass, 2007), the consistency in a suspect's reporting of an alibi (Culhane \& Hosch, 2012), and the evaluator's experience of generating an alibi (Olson \& Wells, 2012) influence evaluators' willingness to accept an alibi. There is also research that suggests that the content of the alibi statement itself (e.g., setting, activities) may influence perceptions of an alibi (Allison, Michael, Mathews, \&

Overman, 2011; Cowan, 2012; Jung, Allison, \& Bohn, 2013). The research on alibi evaluation is at an early stage, so there is a need for replication and for integration of the various research findings. The question of alibi content and its connections to more well established effects is particularly under-researched in the literature, and, as a consequence, formed the central theme of the project as conceptualized. In particular, the current dissertation aimed to replicate the current findings regarding alibi content and to explore the potential interactive influences of alibi content, evidence strength, and alibi provider characteristics on willingness to believe an alibi.

\section{The Problem of Wrongful Conviction (or 11,600 Years of Solitude)}

As of July 19, 2016, The Innocence Project reports 342 exonerations in the United States based on DNA evidence. Their database has allowed researchers to examine the factors that likely led to erroneous convictions, including: eyewitness misidentification, false confessions, police or prosecutor misconduct, the use of un-validated forensic evidence, reliance on deceptive jailhouse informants, and inadequate or negligent defense counsel. It is worth noting that DNA testing is only available in a small percentage of criminal cases (Gross, Jacoby, Matheson, 
Montgomery, \& Patel, 2005; Scheck, Neufeld, \& Dwyer, 2000; Reiss, 2015), so it is unclear whether The Innocence Project's cases are representative of all wrongful conviction cases.

The National Registry of Exonerations lists 1,851 exonerations as of mid-July, 2016, a rather dramatic increase from November, 2013, when the number was $1,241^{1}$. The figures from 2013 show that exonerees spent more than 11,600 years in prison for crimes they did not commit and approximately half spent at least ten years in prison (National Registry of Exonerations, 2013). Unlike the cases from The Innocence Project—which are also included in this databasein the majority of these cases (70\%), DNA evidence did not contribute to the exoneration (National Registry of Exonerations, 2013). Although the proportion of cases that are related to previously identified precursors of false conviction (e.g., eyewitness misidentification) vary between the two databases, the same precipitating factors appear in both.

False convictions have also occurred in Canada. Innocence Canada (formerly The Association in Defense of the Wrongly Convicted), has secured 21 exonerations to date - plus four more in which they had a supporting role - many of which were due to improper testimony by the now-disgraced pathologist Dr. Charles Smith. In April, 2015, they reported that they were actively working on 16 further cases, with an additional 78 cases under review.

In 1972, Romeo Phillion was convicted of the 1967 murder of Leopold Roy, an Ottawa firefighter. Phillion confessed to the murder while being interviewed regarding an unrelated robbery, but immediately recanted his statement. During their investigation, police uncovered a police report that placed Phillion at a gas station in Trenton, Ontario, $288 \mathrm{~km}$ from Ottawa, only two hours before the time of Roy's murder. If the report was true, Phillion could not have committed the murder. Despite this, police pursued Phillion as their prime suspect, and did not

\footnotetext{
${ }^{1}$ The increase does not necessarily represent exonerations that occurred between 2013 and 2016; some of the exonerations were granted earlier, but the cases have only been added to the database recently.
} 
provide this evidence to defense counsel, ostensibly because the gas station report had been proven false in a subsequent police report that has since disappeared. Phillion served 31 years in prison and an additional five on parole after his conviction for Leopold Roy's murder. His case was re-opened in 2006, his conviction was overturned in 2009, and on April 29, 2010, Crown Prosecutors withdrew all the charges against him rather than re-try the case. He lived in this legal limbo - neither proven guilty nor declared innocent - until his death on November 2, 2015.

The data from these organizations have established that wrongful convictions $d o$ occur, and a number of precipitating factors have been identified. But how often do wrongful convictions occur? In the United States, the proportion of criminal convictions that are in error has been estimated to fall somewhere between $0.5 \%$ and $20 \%$ of all cases (Gross et al., 2005; Huff, Rattner, \& Sagarin, 1986; Liebman, Fagan, \& West, 2000; McCloskey, 1989; Poveda, 2001; Ramsey \& Frank, 2007). It is difficult to get a precise estimate (Ramsey \& Frank, 2007), though Reiss (2015) suggested 2.5-5\% is a reasonable approximation. As Ramsey and Frank (2007) noted, even a comparatively conservative estimate suggests a large number of wrongful convictions; a wrongful conviction rate of $1 \%$ in the United States translates to 20,000 innocent people in prison in that country alone.

However, the utility of existing databases for examining rates and causes of wrongful convictions overall is unclear, due to the overrepresentation of murder and sexual assault in exoneration cases (e.g., Gross et al., 2005). These crimes are more likely to yield DNA evidence than others, like robbery (Gross et al., 2005; Kassin \& Gudjonsson, 2004). Interestingly, in the United States, false convictions are more likely to be found in capital murder cases (i.e., a murder for which the perpetrator is eligible for the death penalty; examples include murdering an on-duty police officer or firefighter, or a murder committed while committing another felony 
offense) than for other homicides (Gross et al., 2005). False convictions may genuinely be more common for murder — and particularly capital murder — than other crimes, or this may be an artifact because errors are more likely to be discovered due to the extra attention that these convictions receive (Gross et al., 2005; Harmon, 2001). The intense media exposure in capital cases may result in increased pressure on police to solve the case, and therefore more pressure to use coercive strategies to secure confessions. There also may be a particularly strong incentive for perpetrators to implicate others (e.g., by informing on them to police) to avoid a possible death penalty (Gross, 1996; Gross et al., 2005). Finally, exoneration is a lengthy process, occurring on average ten years after conviction (Gross, 2006), meaning that individuals with long sentences for serious crimes are particularly likely to seek it out. There may be many costs and few benefits to exoneration if one has already served a full sentence and been released.

Interestingly, many actors within the legal system appear to believe that false conviction is not a pressing problem that requires systemic change. In Ramsey and Frank's (2007) survey, defense lawyers (1-3\%), judges (0.5-1\%), and police and prosecutors (less than $0.5 \%)$ estimated low false conviction rates in their jurisdictions. Estimates for national rates followed the same pattern as within-jurisdiction responses, but the numerical estimates were higher. Smith, Zalman, and Kiger (2011) extended this work, finding that defense attorneys expected the greatest number of specific errors (e.g., prosecutors knowingly using false testimony, deliberate or accidental eyewitness misidentification, defense counsel failing to challenge forensic evidence), followed by judges, and then police and prosecutors. As well, they found that police (90.4\%), prosecutors $(72.7 \%)$, and judges (69.9\%) reported that false conviction does not occur with sufficient frequency to merit recommending procedural changes to the justice system. The majority of defense lawyers (91.6\%) disagreed, opining that procedural changes are warranted 
due to the number of erroneous convictions.

It is clear from the investigations of The Innocence Project and The National Registry of Exonerations that wrongful convictions do occur. Social scientists have investigated many of the proposed causes of these errors, including mistaken eyewitnesses and false confessions, and have made recommendations for procedural changes to minimize the likelihood of erroneous convictions. One area that has received relatively little attention is judgments regarding the credibility of alibis. As Steven Avery's case illustrates, disbelieved true alibis may also contribute to erroneous convictions. It is highly probable that Avery was, in fact, at his parents' home and then shopping in Green Bay during the time of Penny Ann Beerntsen's assault. But, given that he was convicted of that crime, it is clear that neither the police nor the jury found his statement sufficiently credible. Due to cases like Avery's, researchers have begun to investigate what constitutes a 'good' or effective alibi.

\section{What is an Alibi?}

The Latin 'alibi' means “elsewhere” (Duhaime, n.d.). In the legal context, an "alibi” generally refers to a claim that an individual was somewhere else while the crime was being committed (Burke, Turtle, \& Olson, 2007), rendering it physically impossible for that individual to be the perpetrator. Burke et al. (2007) identified three types of alibis: true, fabricated, and mistaken. In a true alibi, the alibi provider gives an accurate account of his or her whereabouts. In contrast, fabricated and mistaken alibis are both factually incorrect (i.e., the provider was not where s/he claimed to be), but for different reasons. With a fabricated alibi, the provider deliberately provides misinformation regarding his/her whereabouts. This could be due to the provider's guilt, a desire to protect another person, or to avoid admitting to a misdeed of some kind (Burke et al., 2007). Unlike fabricated alibis, mistaken alibis are honestly offered. In this 
case, an inaccurate alibi has been provided due to a memory error rather than the deliberate intention to mislead. Research by Cowan (2012) showed that undergraduate participants generally do not demonstrate an accurate understanding of the term "alibi."

Case law in Canada has considered both the definition and use of alibis. In the Supreme Court of Nova Scotia decision in $R v$ Heynes in 1914, Justice Townshend wrote that "an alibi means proof of the absence of the accused at the time the crime is supposed to be committed, satisfactory proof that he is in some place else at the time." In 1983, again for the Supreme Court of Nova Scotia, Justice Macdonald wrote in $R v$ Gottschall that

A prisoner or accused person is said to set up an alibi when he alleges that at the time when the offence with which he is charged was committed he was elsewhere. That is, at a place so far distant from that at which it was committed that he could not have been guilty $^{2}$.

Given that it would be impossible for an alibi to be true and the suspect or defendant to be the culprit, an alibi has the potential to be a powerful piece of evidence for innocence. In their 2002 decision in $R v$ Hibbert, the Supreme Court of Canada accepted a set of requirements for jury instructions with regard to the use of alibi evidence in criminal cases. The case involved a real estate agent who was attacked during an open house. The evidence against the defendant, Kenneth R. Hibbert, was largely circumstantial, based on the victim's recollection of details about the perpetrator's life, and included a cap found hanging on a tree along the assailant's escape route that included DNA from Hibbert and the victim (Makin, 2002). Hibbert claimed to have been out with one of his stepdaughters at a cadet flag-raising ceremony that afternoon, before returning home. His alibi was corroborated by two stepdaughters and his wife. Thus, a central issue for the jury at the initial trial was determining whether the alibi was credible. Hibbert argued that the judge erred when informing the jury that they could infer guilt from a

\footnotetext{
${ }^{2}$ See also $R v$ Demers.
} 
disbelieved alibi, even in the absence of evidence that the alibi was deliberately fabricated. The Supreme Court found that the trial judge's instructions were in error, but Hibbert was not granted a new trial due to the curative proviso of s. 686(1) of the Criminal Code.

The Court's decision in $R v$ Hibbert [2002] included four important points regarding the use of alibis in criminal proceedings. First, if the jury determines that an alibi is true, they must return a verdict of not guilty. Second, if the jury does not accept the alibi, but has reasonable doubt about it, they must give a not guilty verdict. Third, if the jury rejects the alibi, they must render a verdict on the basis of all of the evidence provided during the trial. And finally, a rejected alibi can only be used to infer guilt if there is evidence of deliberate deception on the part of the accused; otherwise, the alibi is without evidentiary value. Evidence that alibi witnesses (i.e., individuals corroborating the alibi provided by the defendant) deliberately fabricated their accounts without the knowledge of the accused cannot be used to imply guilt ${ }^{3}$. All in all, an alibi has the potential to be an extremely powerful and influential piece of evidence for innocence. However, alibis may fail to protect innocent people from wrongful conviction.

\section{The Practical Utility of Alibis for Protecting Innocent Suspects and Defendants}

Approximately $25 \%$ of The Innocence Project's first 157 cases included a 'weak' or no alibi as a contributing cause of false conviction (Burke et al., 2007) ${ }^{4}$. Brandon Garrett (2011) conducted a thorough analysis of the first 250 Innocence Project cases, having obtained trial transcripts for 207 of them. Overall, in $67.6 \%$ of the cases that went to trial and there was sufficient information to identify defense strategy, an innocent defendant raised an alibi in court that failed to prevent a conviction, presumably because the alibi was not considered to be sufficiently credible. Given that these individuals were innocent, it is highly likely that the vast

\footnotetext{
${ }^{3}$ For this last point, see also B.C. Court of Appeal decision in $R v$ Tessier.

${ }^{4}$ Interestingly, The Innocence Project does not track alibi issues specifically (E. West, personal communication, May 16, 2011). Poorly investigated alibis may be classified as police misconduct (Burke et al., 2007).
} 
majority of the alibis were honestly offered. Even apparently iron clad alibis sometimes fail to protect innocent suspects; in unrelated cases, Jose Garcia and Richard Alexander were convicted of crimes that occurred while they were in prison (National Registry of Exonerations, n.d.; The Innocence Project, n.d.c.). So although an alibi is intended to prevent wrongful prosecution or conviction, it appears that, at least in some instances, the alibi is a paper shield and provides inadequate protection to innocent suspects. In these two cases, as in many others, alibis were discounted in favour of eyewitness identifications that we now know were incorrect, either due to an error or coerced false testimony.

Comparison of eyewitness and alibi testimony. There are many similarities between alibi and eyewitness testimony (e.g., Burke et al., 2007); both are evidence from an individual seeking to situate a specific person at a specific location at a specific time (e.g., El-Sibaey, 2009). For both types of testimony, there are questions regarding witness credibility and accuracy as well as whether the conditions are favourable for witness recall (Burke et al., 2007). However, alibis appear to be viewed with skepticism (e.g., Cowan, 2012), whereas eyewitness identification is one of the most compelling pieces of evidence that can be presented at trial (Overbeck, 2005). Eyewitnesses — particularly confident ones—are generally believed (e.g., Boyce, Beaudry, \& Lindsay, 2007; Kassin, Rigby, \& Castillo, 1991; Lindsay, Wells, \& Rumpel, 1981; Semmler, Brewer, \& Douglass, 2012), though eyewitness identification is fraught with error (e.g., Brigham, Maass, Snyder, \& Spaulding, 1982; Devenport, Penrod, \& Cutler, 1997; Krafka \& Penrod, 1985; Palmer, Brewer, \& Weber, 2010).

Burke et al. (2007) noted four important differences that likely make recall in the alibi context more difficult than recall in the eyewitness context. First, crime witnesses are likely aware at the time that an important event is occurring, and that later recollections may be 
important $^{5}$. Due to their non-involvement in the crime, innocent alibi providers (i.e., individuals generating an alibi) would be unaware at encoding that their recall of events will be important. Second, innocent alibi providers were likely involved in the mundane activities typical of an average person's day. This is consistent with Garrett's (2011) finding that most Innocence Project exonerees reported being at home with family. Additionally, $35.9 \%$ of participants who were asked to provide a truthful alibi to Culhane, Kehn, Horgan, Meissner, Hosch, and Wodahls (2013) indicated they were at home with friends or family. Third, the honesty of eyewitnesses is generally taken for granted by investigators and jurors, but the honesty of alibi providers and alibi witnesses is regularly questioned. Police or jurors likely assume that the majority of eyewitnesses would not have an obvious motive to provide a false report ${ }^{6}$, but may be more willing to consider that an alibi witness could conceivably offer a false statement to protect a friend or relative. Jurors apparently do not consider the possibility of an eyewitness being pressured by investigators to implicate a particular suspect. Finally, it is generally expected that an alibi should be corroborated with physical evidence or witness testimony, but an eyewitness account is usually treated as evidence in and of itself. In addition, differences in police interview strategies between suspects (i.e., individuals who need to provide an alibi) and witnesses may affect recall. Investigators are likely to use specific techniques to assist witnesses' recall. This is unlikely with a suspect, as interrogation in North America generally prioritizes securing a confession over information gathering (Leo, 2008).

The empirical study of alibis has used Olson's (2002) framework, as it is useful way of organizing the existing literature. In the Generation Domain of the framework, an alibi is

\footnotetext{
${ }^{5}$ In some types of crime, for example fraud, witnesses may often be unaware at the time that a crime is occurring. These eyewitnesses would be expected to suffer from many of the same memory challenges as alibi providers and alibi witnesses.

${ }^{6}$ It is entirely possible that a supposed eyewitness may have reason to implicate an innocent individual, for example to exact retribution for a previous misdeed, but it is unlikely that this is the case for the majority of eyewitnesses.
} 
provided (Story Phase), then evidence that may support it is collected (Validation Phase). In the Believability Domain, the alibi and corroborating evidence are evaluated in the context of all of the available evidence. First, the alibi and evidence are scrutinized by individuals who come into contact with it, including members of law enforcement, judges, journalists, and members of the public (Evaluation Phase). Second, if the case goes to trial, a more formal assessment of the alibi is conducted by the jurors or judge and a decision about the guilt or innocence of the accused is made (Ultimate Evaluation Phase). The sections below will discuss the framework itself in greater detail and demonstrate how general principles from cognitive and social psychology are relevant to the alibi context. The Believability Domain is particularly relevant to the three dissertation studies and will be emphasized.

\section{Alibi Generation Domain}

Story phase. Alibis are only useful in classifying guilty and innocent suspects if innocent individuals can produce accurate, detailed alibis that are supported by compelling evidence; if they cannot, alibis are of little value (Olson \& Charman, 2012). However, when undergraduate participants provide alibis, investigate them, and re-report their alibis at a second session, many alibis change substantially. Olson and Charman (2012) reported that participants were remarkably willing to provide alibis (i.e., give memory statements) and discuss corroborative evidence in the initial session (88\%) and again 48 hours later (92\%). Over a third (36.6\%) of participants' alibis changed between sessions, either in terms of narrative aspects of the alibi $(11.5 \%)$ or in terms of the evidence they could provide to support the alibi while the narrative remained the same (24.9\%). Narrative changes were more frequent when the alibi was for 6-14 weeks earlier (18\%) than when it was three days earlier (4\%), but changes to the corroborative evidence occurred equally at both time periods. These results suggest that a 
substantial number of the initial alibis were inaccurate. It is worth noting, however, that these findings may underestimate the proportion of alibi providers who made errors, as this paradigm does not establish the accuracy of 'accurate' alibis; some participants may have provided the same inaccurate alibi at both experimental sessions.

In a similar study, Strange, Dysart, and Loftus (2014) asked participants to provide a 6hour alibi for themselves for a time three weeks in the past and rate the phenomenological characteristics of their memory using the Memory Characteristics Questionnaire (i.e., confidence, vividness, perspective, and reliving) as well as identifying whether they 'remember' (i.e., have a specific recollection of the event or detail) or 'know' (i.e., believe that an event or detail is accurate, but without a specific recollection) the particular details. The alibi was in the form of responses to seven specific questions, such as: "What did you do?" "What time did each event occur?" and "Who was there?" After spending a week investigating their alibis, participants returned and completed the same measures. Fewer than half of the participants were completely consistent on any of the seven prompts. Inconsistencies were far more common for when things occurred (44\% of participants) than for what occurred (19\% of participants).

Alibi generation requires individuals to access their memories ${ }^{7}$. Autobiographical memory, the memory of events experienced by or related to the self (Matlin, 2005), is one component of long-term memory. Long-term memory comprises three processes: encoding, retention, and retrieval. For a true alibi, the relevant events must enter the long-term memory system during encoding, be stored for an indefinite period of time, and the alibi provider must reconstruct the memory when asked by police. Issues at any of these three processes may hamper an innocent individual's ability to generate an accurate, detailed alibi.

\footnotetext{
${ }^{7}$ This applies to all true and mistaken alibis. As well, in the case of fabricated alibis, providers may be accessing autobiographical memories for other events to report as an alibi.
} 
Encoding. During encoding, information is acquired through the sensory organs and enters memory (Buckner, 2000). Attention and the physical capacity of the sensory organs are a primary determinant of what information is encoded and what is not. Attention is a limited resource, but only information that is attended to and processed in working memory is likely to enter the explicit memory system (e.g., Baddeley \& Hitch, 1974). Attention depends on an event's "novelty, sensory characteristics, $[\ldots]$ personal relevance or salience, emotional meaning and valence, and features of the witness, such as his or her motivation to remember the event" (Read \& Connolly, 2007, p. 122). The mundane events that are likely to comprise an innocent individual's alibi are precisely the type of experiences that do not command attention, for example: a typical work or school day, exercising at the gym, watching television, and cooking. All of these events likely suffer from the effects of low novelty, salience, emotional content, and motivation to remember and are relatively less likely to be attended to and encoded. If the events are not encoded or are poorly encoded, an innocent person is unlikely to provide an accurate alibi that includes sufficient details to be judged as credible by investigators. Alibi witnesses' memories are equally likely to suffer the effects of poor encoding; even if a suspect presents an accurate alibi, witness corroboration might be unobtainable (Burke et al., 2007).

Retention. After information is encoded, it is retained for later retrieval. The Ebbinghaus Forgetting Curve (Ebbinghaus, 1885/1964) describes a rapid loss of encoded information, followed by a more gradual forgetting for an extended period of time (Wixted \& Ebbesen, 1991), due to lack of retrieval cues or interference from memories acquired before or after the relevant event. Innocent suspects may be required to remember events that occurred months or years earlier. Among individuals later exonerated by The Innocence Project, trials took place on average 15 months after the crime occurred (Garrett, 2011), though suspects may have provided 
alibis to police at an earlier time. As well, the details of a routine day are relatively unlikely to be rehearsed, elaborated upon, and discussed with others, which makes them less likely to be recalled later (Read \& Connelly, 2007). Schema-inconsistent details decay more rapidly than schema-consistent or schema inconsistent details in the eyewitness context (Tuckey \& Brewer, 2003). So any unusual features of a particular event may be more likely to be forgotten.

During retention, memories are also vulnerable to distortion from post-event suggestion. In their seminal study, Loftus and Palmer (1974) demonstrated that leading questions influence subsequent recall. Participants' speed estimates for cars involved in an accident were higher when the question used the verb smashed than when hit was used instead. A week later, participants in the smashed condition were more likely than those in the hit condition to erroneously report seeing broken glass in the video. Memories that have begun to decay during normal forgetting are particularly vulnerable to this type of distortion (Loftus, Miller, \& Burns, 1978).

Memory distortions have also been observed for naturalistic autobiographical memories, including recall of the events of the O.J. Simpson verdict. O.J. Simpson, a former professional football player, was tried for the murder of his ex-wife, Nicole Brown Simpson and her friend Ronald Goldman. Simpson was acquitted of all charges after his lawyers systematically discredited the majority of the prosecution's evidence. In USA Today, Price and Lovitt (1997) noted that this was "the most publicized murder case in history" (n.p.). Three days after jurors reached a verdict, Schmolck, Buffalo, and Squire (2000) administered questionnaires to a group of participants that asked an open-ended question regarding how they heard about the verdict and included several specific questions (e.g., what time they heard about the verdict, where they were, and who else was present). A subset of participants was tested again either 15 or 32 
months later. At 15 months, 50\% of the responses were highly accurate (i.e., consistent), compared to $28.6 \%$ at 32 months. Major memory distortions were much more common at 32 months (40\% of low accuracy scores) than at 15 months (10.7\%). Neisser and Harsch (1992) found similar results three years after the Challenger explosion; over $40 \%$ of participants provided inconsistent responses across the two questionnaires. Given the retrieval intervals in alibi generation, many innocent suspects may have forgotten their whereabouts, or may provide incorrect information due to memory distortions.

Retrieval. In retrieval, events are reconstructed from long-term memory. Explicit retrieval generally requires cues, though they may not be easily accessible (Burt, Kemp, \& Conway, 2004). Even if the details of the event were encoded and retained over time without modification, an innocent suspect may lack sufficient retrieval cues to produce an adequate alibi without warning under police questioning.

Schemas affect what details of an event are retrieved (e.g., Brewer \& Nakamura, 1984; Taylor \& Crocker, 1981). Greenberg, Westcott, and Bailey (1998) showed participants a set of slides of a convenience store robbery, based on an initial study identifying elements of individuals' scripts for robberies, that either omitted three central details (i.e., mentioned by at least $75 \%$ of the initial participants) or three peripheral details (i.e., infrequently mentioned by initial participants). Participants were significantly more likely to erroneously report having seen omitted central details than peripheral details, particularly for longer retention intervals. Participants used their robbery schemas to fill in their recollections and reported seeing actions that they did not actually witness. When innocent alibi providers who have weak memories for the relevant time, they may fill in gaps in their memories with plausible schema-consistent details or may combine details from several genuine events (Olson \& Charman, 2012). 
Additionally, individuals with regular schedules may rely on schemas and report what they typically do at a particular time of the week rather than what they actually did (Charman, Cahill, Leins, \& Carol, 2010).

Due to the challenges at encoding, retention, and retrieval, many innocent suspects will likely have difficulty generating an accurate, detailed alibi. These challenges are compounded by misperceptions regarding how memory works (e.g., the memory-as-videotape metaphor), and the influence of motivation. Individuals expect that motivation at encoding and at recall facilitates memory; however, only motivation at encoding enhances later recall (Kassam, Gilbert, Swencionis, \& Wilson, 2009). Describing their results, Kassam et al. (2009) noted that "judges mistakenly expected memorizers to remember information that became important as though it had always been important" (p. 552). For innocent alibi providers, this presents a problem, as motivation will be present at recall, but not at encoding.

Validation phase. The strength of an alibi is determined in the Validation Phase, where physical evidence and person evidence (i.e., witness testimony) that supports the alibi is collected (Burke et al., 2007). Alibi strength is operationalized as the ease with which evidence could be fabricated (Olson \& Wells, 2004). For example, a credit card receipt would be easier to fabricate than CCTV footage would be. Fabrication of person evidence involves recruiting someone to lie to support an alibi. It is generally expected that friends or family members are more likely to corroborate a false alibi than a stranger (e.g., Hosch, Culhane, Jolly, Chavez, \& Shaw, 2011) because friends and family members have a vested interest in keeping their loved ones out of prison, though this assumption has been recently questioned (e.g., Marion \& Burke, 2013).

Olson and Wells (2004) created a taxonomy of alibi strength based on ease of fabrication. 
Participants each rated the believability of alibis of three suspects in an armed robbery investigation. Four levels of person evidence (i.e., none, non-motivated stranger, non-motivated familiar other, motivated familiar other) were manipulated between participants and three levels of physical evidence (i.e., none, easy to fabricate, difficult to fabricate) were manipulated within participants. Results indicated that the quality of the physical evidence was the primary determinant of believability ratings. Stronger physical evidence led to higher believability ratings at all levels of person evidence except the non-motivated stranger. Even relatively weak physical evidence was sufficient to negate any influence of witness corroboration; the quality of the person evidence only affected believability when there was no physical evidence to support an alibi. In this condition, alibis supported by non-motivated familiar others (e.g., your regular barista) or strangers were rated as more believable than alibis that lacked person evidence. Alibis corroborated by strangers, but not non-motivated familiar others, were also more believable than alibis corroborated by motivated familiar others (e.g., your mother). Finally, the trend was for stranger-corroborated alibis to be more believable than non-motivated familiar other-supported alibis. Participants neglected the fact that strangers can be mistaken.

Person evidence. Other research on how alibi witness corroboration affects alibi believability has generally supported Olson and Wells' (2004) findings that corroboration by a non-motivated other is more powerful than corroboration by a motivated other. In Lindsay, Lim, Marando, and Cully's (1986) mock assault and robbery trial study, the number of guilty votes was significantly lower when the defendant presented an alibi corroborated by a stranger (27\%) than in a no-alibi control condition $(60 \%)$. However, corroboration of the alibi by the defendant's brother-in-law yielded a rate of guilty votes $(57 \%)$ that was not significantly different from the control condition. Similarly, in their armed robbery investigation scenario, Culhane and Hosch 
(2004) found significantly lower guilty verdicts when the alibi was corroborated by a neighbour who was certain compared to when the alibi was uncorroborated. However, corroboration by the defendant's girlfriend did not reduce the rate of guilty verdicts compared to the no-corroboration control. It is worth noting that both of these studies examined participants' reports of their behaviour in a hypothetical scenario and did not include a measure of actual behaviour. To address this limitation, the first two studies in this dissertation included measures of explicit behaviour.

Hosch et al. (2011) asked participants to estimate the probability that 15 different potential alibi witnesses would lie for a defendant. Participants reported that biological relatives were more likely to lie than those who were related by marriage, who were, in turn, more likely to lie than those with only social bonds to the defendant. Within the three categories (biological, marriage, social relationships), individuals with a closer relationship were anticipated to be more willing to lie than those with a more distant relationship (e.g., sibling vs. second cousin, best friend vs. acquaintance seen often at the store). These patterns were not influenced by participants' estimates of the degree of contact between the defendant and alibi witness. The same patterns were observed when the participants were asked to report their own willingness to knowingly corroborate a false alibi for different people. In general, it seems that when there is a close relationship between the provider and witness, particularly when they are biologically related, alibis generally do not reduce the rate of guilty verdicts compared to when a suspect or defendant offers no corroboration (Culhane \& Hosch, 2004; Hosch, Culhane, \& Howley, 2005; Olson \& Wells, 2004) or no alibi at all (Lindsay et al., 1986). Alibi witnesses with relatively more distant relationships to the provider appear to be the most—or only—effective corroborators. 
What evidence is typically available to defendants? The key determinant of alibi strength is physical evidence (Olson \& Wells, 2004). Unfortunately, such physical evidence may frequently be unobtainable. Many criminal defendants in Canada and the United States raise alibis at trial (up to $86 \%$ of court cases), but few (14\%) are corroborated with physical evidence (Burke \& Turtle, 2003). In the exoneration cases discussed by Garrett (2011), 86.4\% of the alibis (121 of 140 alibis) were supported by witnesses, primarily family members testifying that the defendant was at home with them at the time of the crime, and almost none provided physical evidence to support their alibis. Unless an alibi provider lived in an apartment or condo building with security cameras in the lobby and hallways, it is difficult to imagine what physical evidence an individual could provide to support an alibi that s/he was at home. Corroboration from a nonmotivated other is also unlikely in this scenario. As Olson and Charman (2012) pointed out, some alibi narratives are inherently impossible to corroborate.

These findings are consistent with the empirical literature. The vast majority (81\%) of the alibis presented in the Olson and Charman (2012) study discussed earlier were corroborated by no or very weak evidence. Participants expected to find strong physical evidence to support only $9 \%$ of their alibis, and witness corroboration from a non-motivated familiar other or stranger for only $6 \%$ of their alibis. These figures may overestimate the strength of the evidence that is actually available; participants reported the evidence they expected would be available, but were not required to actually collect it. Witnesses may have forgotten the event in question and physical evidence could be lost. Strange, Dysart, and Loftus (2014) reported similarly poor corroboration; $47 \%$ of their undergraduate participants were unable to provide any evidence whatsoever to corroborate their alibis, and none of the participants could find a non-motivated stranger as an alibi witness. In general, it appears that the majority of alibis are likely to be 
considered weak by evaluators (Burke \& Marion, 2012; Burke et al., 2007; Olson \& Charman, 2012), and these alibis are unlikely to be viewed as credible (e.g., Dahl, Brimacombe, \& Lindsay, 2009; Lindsay et al, 1986).

Certain demographic characteristics of the alibi provider may result in that person being more likely to provide a weak alibi (Burke et al., 2007). Someone who is married, employed, and has an active social life outside of the home is more likely to provide an alibi that can be strongly corroborated than someone who is single, unemployed, and not particularly sociable. Being at work provides many opportunities for corroboration—-for example security video, keycard access records, and statements from coworkers or clients - that would be unavailable to someone who is unemployed or works at home. This issue is apparent in The Innocence Project's case files; the majority of their first 250 exonerees were unemployed at the time of the relevant crime, which contributed to them having weak alibis (Garrett, 2011).

Overall, the current research on alibi generation suggests that it is difficult for innocent individuals to provide strong alibis for themselves. The relevant events may have been poorly encoded, may have decayed or been altered during retention, and may be difficult to retrieve. This is the case for both alibi providers and alibi witnesses (Burke et al., 2007). These findings are relevant to the issue alibi content, which is a central theme in the current work; difficulties with recall may result in alibis that are inaccurate, or are lacking in detail. In addition, those who are able to provide alibi narratives for themselves may be unable to produce strong evidence to support them (e.g., Burke et al., 2007; Olson \& Charman, 2012). Corroboration by a motivated other is more common than corroboration by a non-motivated other, but is unlikely to provide much tangible benefit to a suspect or defendant. Physical evidence is much more influential than person evidence, but is only rarely available. Unfortunately, this would likely result in alibis that 
are rejected by evaluators, a factor that is also investigated in the three studies in this work.

\section{Alibi Believability Domain}

Once an alibi has been generated and the corroborative evidence has been collected, the alibi is evaluated by those who encounter it in Olson's (2002) Believability Domain, which includes the Evaluation and Ultimate Evaluation phases. During the Evaluation Phase, the alibi is scrutinized by a number of people, including police officers investigating the case, Crown attorneys determining whether to prosecute the case, defense lawyers, journalists covering the investigation, and members of the public who are exposed to pre-trial publicity. If the case proceeds to trial, during the Ultimate Evaluation Phase, a final determination is made regarding the credibility of the alibi and the guilt or innocence of the accused. In both of these phases, judgments regarding alibi believability are made in the context of all of the available information

(e.g., witness identifications or non-identifications, forensic tests). There is a small, but growing, literature on the factors that influence alibi believability.

General skepticism and the alibi generation effect. In general, alibis appear to be viewed with skepticism. Steven Avery was convicted despite a strong alibi. The strongest alibi that Olson and Wells (2004) presented to participants, which included corroboration from a neutral person familiar with the suspect as well as security video with a time/date stamp, was only rated 7.4 out of 10 for believability. The authors wondered what type of corroboration would be required for a higher rating, or whether a higher rating was even possible. They suggested that use of the term 'alibi' might lead to evaluator skepticism. Alibis are only collected from criminal suspects; their statements may already be tainted by the suspicion of being a suspect (Burke et al., 2007). Cowan (2012) found that the same set of statements was judged as significantly less believable when they were described as potential alibis than when they were 
described as typical memory reports.

At least two other factors may contribute to this skepticism about alibis. First, evaluators may overestimate the quality of autobiographical memory (e.g., Burke et al., 2007; Olson \& Wells, 2012). Overbelief of one's own performance has also been found for eyewitness identification tasks (e.g., Hourihan, Benjamin, \& Liu, 2012; Read, 1995, Experiment 3) and other types of memory tasks, such as memory for words (e.g., Bjork, Dunlosky, \& Kornell, 2013; Zimmerman \& Kelley, 2010). When alibi providers generate alibis that do not meet the evaluators' unreasonably high expectations for autobiographical memory, this may seem suspicious.

Olson and Wells (2012) hypothesized that having individuals generate their own alibis before evaluating someone else's might sensitize them to the difficulty of providing an alibi supported by strong evidence and result in greater acceptance of moderately strong alibis. They conducted two experiments in which participants were asked to evaluate a "moderately" strong alibi (i.e., no physical evidence, corroboration by a friend), either after having generated their own alibi or before doing so. In both studies, participants who began by providing their own alibi rated the suspect's alibi as more believable than participants who generated their alibis later. Their second study also showed that merely reading about the difficulties of alibi generation led to ratings that were not significantly different from ratings of those who generated alibis before evaluating or who generated alibis later. The researchers suggested that the alibi generation effect is partly due to acquiring the knowledge that alibi generation is difficult, but that the alibi generation experience is necessary to produce significant changes in alibi believability ratings.

The second explanation for skepticism is that evaluators may have the perception that alibis, when provided as evidence in criminal cases, should be carefully scrutinized, unlike 
typical memory reports. According to Gilbert (1991), understanding and acceptance of information are the same cognitive process. When individuals encounter new information, it is always immediately accepted as true. If motivation and sufficient cognitive resources are present, the information is later subjected to a more labour-intensive evaluation process in which the information is unaccepted if it is deemed false. Alibis may be more likely to be subjected to this second phase than are memory reports in other contexts because there is a more obvious motivation to lie (i.e., avoiding punishment) than there is in other contexts. Due to this additional scrutiny, evaluators may be more likely to question aspects of the statement (e.g., why would s/he be jogging at that hour?) or attend to inconsistencies in the statement.

Alibi change. As discussed earlier, research participants' alibis often change after investigation (e.g., Olson \& Charman, 2012). The prevalence of alibi change varies across studies, from 30\% (Olson \& Wells, 2003) to over 50\% (Strange, Dysart, \& Loftus, 2010). Unfortunately, there is a general assumption that an innocent suspect's alibi should not change (Strange, Dysart, \& Loftus, 2014), and police often interpret inconsistencies between statements as indicating guilt or deception (Culhane, 2005; Dysart \& Strange, 2012). Although a minority of officers (19.1\%) indicated that alibi change could be due to an initial mistake, they attributed those errors to drug or alcohol use, rather than representing memory failures of average, sober adults (Dysart \& Strange, 2012). However, deception detection research has shown that truth tellers are more likely to correct their initial statements than liars (see meta-analysis by DePaulo, Lindsay, Malone, Muhlenbruck, Charlton, \& Cooper, 2003), so alibi change is more likely to be due to memory error than an attempt at deception.

Culhane and Hosch (2012) found that alibis that remained the same were rated as more believable than alibis that changed. This was the case even when the change resulted in the 
evidence supporting the alibi becoming stronger, though a change to a weaker alibi was particularly damaging in their first—but not second—study. The small difference in results is likely due to differences in methods; in their first study, participants imagined suspects providing alibis during interrogation, whereas in the second study, participants were shown interrogation transcripts. In contrast, Reynolds (2010) did not find significant differences in rates of guilty verdicts comparing a consistent alibi with alibis that changed for a variety of reasons. Given the limited literature and contradictory findings, any effect of alibi change on evaluator judgments remains unclear.

Evaluator role. The context in which an alibi is presented may also influence believability judgments. In their first study, Sommers and Douglass (2007) found that an alibi in a vandalism case was rated as stronger and more credible when it was presented in an investigation context (i.e., as a police report) than in a trial context. When the alibi was presented in a no-context control condition, it was judged as equally strong as the alibi in the investigation context and equally credible as the alibis in the investigation and trial contexts. Participants seem to have assumed that the control condition summary was of an investigation rather than a trial. In their second study, they manipulated the presence of corroboration as well as the context (investigation vs. trial). The alibi was rated as stronger and as more credible in the investigation context than in the trial context. As well, corroboration by the suspect's mother only bolstered perceptions of strength and credibility in the investigation context. They suggested that participants inferred that an alibi that was presented in a trial context had already been judged during an investigation as insufficiently credible. This inference would be less likely in an investigation context, where information gathering is ongoing. They acknowledged the alternate explanation that evaluators used more stringent criteria to assess the alibi in the trial context 
because of the relatively more serious consequences. The decision to investigate or exculpate a suspect in an investigation may be viewed as less critical than a verdict decision in a trial; investigation errors can be corrected at trial, but an error during a trial is not so easily remedied.

\section{Alibi Content}

Research has only begun to address the question of whether the content of an alibi (e.g., settings, activities, narrative features) affects an evaluator's willingness to believe it. Initially, Olson and Wells (2004) argued that while the "surface attributes" (p. 159), like activities or setting, would vary across alibis, these differences were largely irrelevant. They offered no clear theoretical rationale or empirical support for this claim. However, the number of pauses, verbal hedges (i.e., language indicating uncertainty), and amount of spatial detail are associated with judgments about an alibi and the alibi provider (Allison, Michael, Mathews, \& Overman, 2011). Also, when Maeder and Dempsey (2013) manipulated the gender of the defendant and whether s/he offered a stereotypically masculine alibi (shopping for tools) or stereotypically feminine alibi (shopping for skin-care products), they found that the gender of the defendant and the alibi type influenced participants' perceptions of defendant femininity; in particular, female defendants and defendants who provided stereotypically feminine alibis were rated as more feminine. In turn, higher perceptions of femininity led to more trust in the defendant's testimony, more liking of the defendant, and higher belief of the alibi. Finally, belief in the defendant's testimony and impressions of the defendant—but not belief in the alibi-affected verdict composite scores such that greater belief in the testimony and more positive impressions of the defendant were associated with lower guilt ratings. Essentially, the content of the alibi influenced guilt ratings through other intervening variables. Finally, research on schemas, expectations, and implicit personality theory suggest that the events described in an alibi may 
influence how it is perceived and judged. The studies in this dissertation contribute to our understanding of alibi content, particularly how the moral desirability of activities described in the alibi affects credibility judgments.

Moral desirability and alibi content. The online edition of the Oxford English Dictionary (2013) defines the adjective 'moral' as:

of or relating to human character or behaviour considered as good or bad; of or relating to the distinction between right and wrong, or good and evil, in relation to the actions, desires, or character of responsible human beings; ethical.

There is no clear consensus among philosophers regarding how to define terms like 'right' and 'wrong,' how moral decisions should be made, or even what issues are part of the moral sphere ${ }^{8}$. Differences across cultures are also to be expected. An action that is a serious moral transgression in one country may be morally irrelevant in another one (Sverdlik, Roccas, \& Sagiv (2012). Within-country differences are also common; American Republicans and Democrats have very different approaches to morality (Sverdlik et al., 2012). Within psychology, there are also disagreements with how morality and moral development should be conceptualized, for example the differences between Kohlberg (e.g., Kohlberg, 1973), who viewed morality in terms of justice, and Gilligan (e.g., Gilligan, 1977), who emphasized a morality founded on caring for others. As a result of these disagreements and tensions, operationally defining 'moral desirability' is a challenging endeavour. In experimental research about moral desirability, the key consideration is how participants evaluate the morality of activities in the relevant manipulations. Thus, it is participants' subjective impressions, rather than philosophical arguments, that are of primary importance in this context. A manipulation will only be successful inasmuch as participants perceive the activities as morally good or bad. For

\footnotetext{
${ }^{8}$ For example, Kant believed that only beings who were rational (i.e., adult men) were worthy of moral status, Mill included all people as morally relevant, and some more recent branches of philosophical thought now include nature and ecosystems within the moral realm.
} 
the purposes of the current work, a bottom-up approach for identifying morally desirable and undesirable behaviour was chosen. The morality of activities was defined in terms of popular opinion; an action was considered morally undesirable or desirable if the majority of individuals reported that they perceived it as such.

There are two competing theories that purport to explain the effect of alibi moral desirability on legal judgments. These theories predict an opposite pattern of effects, and there is some support in the literature for both. First, an alibi that describes morally questionable activities could lead an evaluator to form a negative implicit personality theory and infer that the alibi provider is morally bankrupt in general, view the provider's statement with added suspicion, and believe a morally undesirable alibi less than a neutral one (Allison, Mathews, \& Michael, 2012). Conversely, it is possible that evaluators take the admission of misdeeds in an alibi narrative as an indication of truthfulness, and rate morally undesirable alibis as more believable than neutral ones (Allison, Mathews, \& Michael, 2012).

Attributions, implicit personality theories, alibis, and legal outcomes. Individuals are constantly making attributions about the causes of others' behaviour to make sense of their social worlds and interact effectively with others (Brehm, Kassin, Fein, \& Burke, 2008; Heider, 1958). Behaviour can provide insight into the beliefs, attitudes, personality characteristics, motivations, and values of others. We may infer that concert attendees like the band's music, that someone who votes for Justin Trudeau possesses liberal economic and/or social values, and that the person who gives up a seat on public transit for an elderly individual is kind and courteous. Behaviour can be elicited by internal factors, as in the examples, or can be prompted by situational forces. For example, an individual may give up a seat on transit because he or she is a kind and courteous individual (dispositional attribution) or due to prompting or perceived judgment of 
other passengers (situational attribution). Dispositional attributions provide greater insight into the character of the actor and are likely anticipated to be most useful in predicting the actor's future behaviour than are situational attributions. Knowing that an individual gave up a seat on the bus out of kindness suggests that s/he will engage in that behaviour again in the future, and may engage in other acts of kindness; no such guarantees regarding kind acts are offered when the seat is relinquished due to perceived external pressures.

Correspondent Inference Theory (Jones \& Davis, 1965) proposes that dispositional attributions are more likely when behaviour is seen as freely chosen by the actor, is unexpected or unusual, and has a single clear outcome. In their second study, Jones and Harris (1967) found that choice and expectedness affected participants' attributions about the attitudes of an individual who wrote a speech about Cuba that was pro-Castro, anti-Castro, or ambivalent. As expected, participants rated the speech as being more consistent with the author's views when the position in the essay was described as freely chosen than when it was assigned. Participants still made attributions about the author's opinion when the perspective was assigned, even though this is illogical (Kelley, 1972). In response to an ambivalent speech, participants assumed that the author held a view that was inconsistent with the position taken in the speech. Participants appear to have inferred that the author disagreed so strongly with the position s/he was supposed to argue that his/her true views leaked into the argument. In terms of expectedness, choice was more influential when the speech content was unexpected (i.e., pro-Castro") than expected; the difference between the chosen and assigned conditions was greater for the proCastro speech than the anti-Castro speech. Similar results have been found with essays about segregation (Jones \& Harris, 1967, Experiment 3; Steiner \& Field, 1960). A dispositional

\footnotetext{
${ }^{9}$ In the United States in the 1960s, a pro-Castro speech would be unexpected, and the participants' own attitudes about Castro and Cuba were generally negative.
} 
attribution is likely for a morally undesirable alibi, as bad acts are generally unexpected and, in the absence of contradictory information, may be assumed to be chosen by the actor.

Humans are very willing to make global judgments about others based on limited information. For example, in the Halo Effect (e.g., Greenwald \& Gillmore, 1997; Guilford, 1954; Nisbett \& Wilson, 1977), knowledge that an individual has one positive characteristic generally leads others to assume that person has other positive characteristics. Physically attractive people are expected to possess many positive traits (Dion, Berescheid, \& Walster, 1972). The reverse is also true: individuals who are known to have one negative trait are expected to have other negative traits as well (Corsini, 1999). As a result, an alibi that describes morally undesirable behaviour would not only result in a single negative attribution about the alibi provider (e.g., the provider is selfish), it would be expected to manifest in a general negative personality theory about the provider (e.g., the provider is also deceitful, unkind, and lazy).

These general attributions influence legal decision-making. Physically attractive defendants receive more not guilty verdicts and shorter recommended sentence lengths than physically unattractive defendants (e.g., Bull \& Rumsey, 1988; Downs \& Lyons, 1991; Efran, 1974; Levelthal \& Krate, 1977; Solomon \& Schopler, 1978; Stewart, 1980). Facial characteristics also influence verdict choice. 'Baby-faced' (i.e., large eyes, round cheeks and chin) adults are more likely than 'mature-faced' adults to be found guilty of negligence, and the pattern is reversed for accusations of deliberate misdeeds (Berry \& Zebrowitz-McArthur, 1988; Zebrowitz \& McDonald, 1991). This is likely because ‘baby-faced' individuals are seen as being naïve and honest, whereas 'mature-faced' people are seen as particularly dominant and competent (Berry \& Zeibrowitz-McArthur, 1988; Brehm et al., 2008). Physical appearance may lead to general attributions regarding a defendant's character, which influence legal decisions. 
Legal decisions are also influenced by the social attractiveness of the defendant and the victim. In their first experiment, Landy and Aronson (1969) found a trend towards longer sentence lengths when the victim in a negligent homicide case was described in pro-social terms (i.e., good job, loving family, history of charitable work) than when the victim described in antisocial terms (i.e., a hoodlum under police investigation, prior convictions, body found with a gun). In their second study, the victim description manipulation was strengthened; the pro-social victim was now a noted architect and philanthropist and the anti-social victim became a notorious Mafioso suspected in a mass killing. A new manipulation was added such that the defendant was also described in pro-social (i.e., friendly and hard-working professional and widower with no criminal history), anti-social (i.e., multiply-divorced manual labourer with previous misdemeanor convictions), or neutral terms. Participants were convinced of the defendant's guilt in all conditions. Again, there was a trend for longer recommended sentences when the victim was socially attractive versus socially unattractive. The anti-social defendant received significantly longer sentence recommendations than the pro-social or neutral defendants, whose sentence recommendations did not differ.

The literature on physical and social attractiveness suggests that exposure to a morally undesirable alibi may lead evaluators to make a negative dispositional attribution about the alibi activities, form a general negative implicit personality theory about the provider (i.e., infer that the provider is generally a 'bad person'), and be more likely to judge the provider harshly compared to when a neutral alibi is offered. This would manifest in lower ratings of alibi believability and higher ratings of probability that the provider is guilty. A morally desirable alibi could lead to a positive implicit personality theory, and therefore higher ratings of alibi believability and lower expectations of provider guilt, though it should be noted that Landy and 
Aronson (1969) did not observe any differences between their pro-social and neutral defendants. Differences between morally desirable and neutral alibis are more likely if the alibi activities are viewed as unexpected and chosen by the actor.

Morally undesirable alibis and the assumption of honesty. Alternately, evaluators may interpret the admission of misdeeds in an alibi narrative as an indication of truthfulness (Allison, Mathews, \& Michael, 2012), due to an assumption that liars are unlikely to offer lies that cast them in a negative light. Speakers tell lies (i.e., intentional false statements, analogous to fabricated alibis) to achieve a specific purpose, including protecting privacy, pleasing an authority, or benefitting one's in-group (see Aune \& Waters, 1994). Individuals may lie to maintain someone else's positive impression of them or to avoid damaging this positive impression (e.g., Aune \& Waters, 1994). With this in mind, it seems unlikely that a liar would invent a story that could lead to embarrassment, loss of social status, and damage to interpersonal relationships.

In a series of three studies, Sternglanz (2004) found that ratings of a target's guilt were lower when the target denied involvement in the offence and also admitted to a lesser relevant offense than when the target merely denied involvement in the offense. In the first study, participants read vignettes describing an academic integrity offense. In the second study, participants watched videos of confederates denying involvement in a variety of transgressions, using one of six different strategies (no response, denial, denial with another explanation for the behaviour, denial and a counter-accusation about the accuser, denial and admission of a relevant lesser offense, and denial and admission of an irrelevant lesser offense). In the third study, participants were videotaped describing a time when they had been accused of a serious lie. In some cases, the accuser was correct (i.e., the participant had lied) and in other cases, the accuser 
was incorrect (i.e., the participant told the truth). These participants were instructed to demonstrate their innocence using one of the six strategies above. The videos were then rated by a second group of participants. In all studies, admitting to a lesser offense reduced likelihood of guilt judgments compared to at least some of the other approaches. Sternglanz (2004) argues that individuals may infer forthrightness from an admission about another misdeed.

The Allison Lab at Elon University has, to date, conducted three experiments investigating alibi "salaciousness" on evaluator judgments, comparing a salacious alibi to a neutral alibi. Salacious behaviour typically involves the violation of social norms, but does not necessarily constitute a moral or ethical violation (e.g., the definition from dictionary.com: "lustful or lecherous. [of writings, pictures, etc.] obscene; grossly indecent"). First, Allison, Mathews, and Michael (2012) provided participants with a transcript of a robbery-homicide case, adapted from Olson and Wells (2004), in which the suspect offered as an alibi either that he had been at home watching an adult film ('salacious') or a regular film ('non-salacious'). The provider either corroborated his alibi with a cable bill and a statement from a neighbour or provided no physical evidence and offered corroboration by his brother. The alibi and the corroborator were rated as more believable, and the suspect was rated as less likely to be guilty when the alibi was salacious compared to neutral, and when it was corroborated by relatively stronger evidence. The authors suggested that evaluators inferred that the suspect's primary motivation was to show his innocence, even if that required admitting to salacious activities.

However, there are some methodological issues that cast doubt on the results. First, the authors" "strong" and "weak" categories are not consistent with Olson and Wells' (2004) conceptualization of strong physical evidence. The cable bill is more accurately categorized as weak corroborative evidence because it does not establish that the suspect watched the film. 
Second, the neighbour could be an unmotivated familiar other or a motivated familiar other, depending on the nature of their relationship, which was not clarified. A neighbour with whom the suspect has a close, long-term relationship could be motivated to corroborate an alibi that s/he knows is false, whereas an acquaintance who happens to live next door would presumably be less likely to do so (e.g., Hosch et al., 2011; Marion, 2014; Marion, 2013). Consequently, the two conditions are more accurately called "stronger" and "weaker" corroborative evidence.

Allison, Jung, Sweeney, and Culhane (2013) found no significant effect for alibi content on verdicts when the alibis involved downloading adult films for illegal distribution, downloading Hollywood films for illegal distribution, or watching a Hollywood film. However, the corroborator was rated as more believable and participants were more certain of their verdicts for the alibi that involved copying Hollywood films than the other two alibi conditions. This result, then, is not particularly consistent with the results of their initial study.

And in their third study, Jung, Allison, and Bohn (2013) manipulated crime type (sexual assault vs. physical assault vs. theft), alibi salaciousness (having an affair vs. having a work dinner) and corroborative physical evidence (receipt vs. no receipt). The manipulation of alibi salaciousness could also constitute a manipulation of moral desirability; an affair is arguably immoral and a work dinner is arguably morally neutral or irrelevant. The alibi was rated as more believable, perceptions of the defendant and corroborator were more positive, and fewer guilty verdicts were obtained when there was physical evidence compared to when there was an absence of physical evidence. Few effects were observed for alibi salaciousness. The defendant and corroborator themselves were rated more positively when the alibi was non-salacious and there was physical evidence to support it than in the other conditions.

Overall, the results for alibi salaciousness have been inconsistent. This may be due, in 
part, to the nature of the 'salacious' activities themselves. Undergraduate participants may not perceive watching adult films or illegal file sharing as particularly salacious, but these are the activities used in the first two studies. No items were included to assess participants' perceptions directly. Although it is unclear whether the affair alibi used in the third study was considered salacious, it seems reasonable to expect that it was viewed negatively (e.g., Cowan, 2012). The current work addressed this limitation by pilot testing a set of possible alibis to identify narratives that were consistently viewed as morally desirable, undesirable, and neutral. Manipulation check items were also included to determine the success of the manipulation.

My Masters research (Cowan, 2012) addressed this issue. In the initial study, participants rated the moral desirability of a set of activities. From these ratings, ten activities were chosen that represented a range from highly morally undesirable to highly morally desirable, with two neutral activities. These activities were presented as alibis to participants in the second study. The moral desirability of the alibi statement did not affect ratings of alibi truthfulness, alibi accuracy, or strength of incriminating evidence. However, it did influence estimates of the likelihood of suspect guilt; the probability that the suspect was the culprit was rated as significantly lower when he reported having sex with a friend's romantic partner (very morally undesirable) than when he was stealing pop from a convenience store (somewhat morally undesirable) or receiving medical treatment for priapism (neutral). This pattern may be explained by considering the imagined state of the victim of an alibi narrative; the cuckolded spouse may be a clearer victim than the shopkeeper and there is no obvious victim with priapism (aside from the alibi provider himself, potentially). Harm to the victim of a crime affects attributions of blame (e.g., Austin, Walster, \& Utne, 1976; Wissler, Evans, Hart, Morry, \& Saks, 1997) and sentence-length recommendations (e.g., Kerr \& Kurtz, 1977; Myers, Lynn, \& Arbuthnot, 2002; 
Scroggs, 1976). Perhaps this same effect applies to the players in an alibi scenario. These findings for moral desirability, along with some of the results from the Allison Lab, lend some support to the theory that reporting engaging in undesirable behaviour as part of an alibi may result in an assumption that the provider is being honest; the suspect that offered one of the very negative alibis, which involved admitting to having harmed someone, was perceived as less likely to be guilty of the criminal offense than in some of the other conditions.

Two additional limitations to the Allison studies and to Cowan (2012) are the absence of a control group that is not provided alibi information and the lack of behavioural measures. All four studies compared only alibis of different types. As such, it is not clear under which conditions an alibi reduces probability of guilt judgments or the proportion of guilty verdicts compared to when a suspect is unable to recall his/her whereabouts and provide an alibi statement. Given the challenges inherent in alibi generation discussed earlier, and the likelihood that many innocent suspects are unable to generate alibis for themselves, this issue is of practical significance as well as being theoretically interesting. As well, it is unknown whether any alibi manipulations influence behaviour. Both limitations are addressed in this work.

Alibi provider characteristics. Stereotypes influence mock-juror decisions (Gordon, Bindrim, McNicholas, \& Walse, 1988; Jones \& Kaplan, 2003; Maeder \& Dempsey, 2013), for example the stereotypes about facial characteristics discussed earlier (e.g., Berry \& ZebrowitzMcArthur, 1988; Zebrowitz \& McDonald, 1991). In North America, stereotypes about racial minorities persist, including against African Americans (e.g., Dixon \& Rosenbaum, 2004; Madon, Guyll, Aboufadel, Montiel, Smith, \& Jussim, 2001), First Nations people in Canada (e.g., Donakowski \& Esses, 1996), and Hispanic people (e.g., Dixon \& Rosenbaum, 2004), cultural stereotypes regarding a variety of nationalities are widespread (e.g., Madon et al, 2001), 
and racial harassment persists (e.g., Bucchianeri, Eisenberg, \& Newmark-Sztainer, 2013). In Canada, the number of racist comments that are posted in response to news articles about Indigenous peoples led the Canadian Broadcasting Corporation - Canada's national public broadcaster - to eliminate the comments pages for all articles about Indigenous issues in late November, 2015. It is apparent that there is, at least, a significant minority of non-Indigenous Canadians who hold strongly negative views towards First Peoples and are willing to make these views publically known through online comments sections, albeit anonymously. It should be noted that in Canada, the term "Indigenous" refers to individuals who are First Nations (i.e., Aboriginal peoples who are not Inuit or Métis), Inuit (i.e., Aboriginal peoples generally from the far north), and Métis (i.e., individuals who are members of cultures that emerged from unions between Aboriginal and European people) (First Nations and Indigenous Studies, 2009).

The issue of racial stereotypes is of particular concern in the legal system. Members of minority groups are disproportionately involved in the criminal justice system. Approximately $60 \%$ (National Registry of Exonerations, 2013) to 70\% (The Innocence Project, n.d.d.) of exonerees in the United States are members of visible minority groups. In Canada, in the 2011 National Household Survey, approximately $4.3 \%$ of Canadians self-identified as Indigenous (First Nations: 60.8\%; Métis: 32.3\%; and Inuit: 4.2\%) (Statistics Canada, 2015). However, $23.2 \%$ of federal inmates are Indigenous people (71\% First Nations, 24\% Métis, and 5\% Inuit), and the incarceration rate for Indigenous individuals is approximately ten times the incarceration rate of non-Indigenous people in Canada (Office of the Correctional Investigator, 2013). The problem is even starker for Indigenous women, who make up 33.6\% of federal inmates (Office of the Correctional Investigator, 2013). Among other causes, the high rate of incarceration of Indigenous people in Canada has been linked to discrimination against and prejudice towards 
them (Office of the Correctional Investigator, 2013).

Race affects verdict choice (e.g., Hodson, Hooper, Dovidio, \& Gaertner, 2005) and the likelihood of receiving the death penalty (e.g., Eberhardt, Davies, Purdie-Vaughns, \& Johnson, 2006). Sommers and Ellsworth's (2001) review showed that, in the US, White jurors treated Black defendants more harshly than White defendants, but only when race is not a salient issue in the trial. They argued that race salience activates social norms to avoid appearing prejudiced among White jurors. When race is not salient, the norms are not triggered and White jurors' judgments are affected by lingering prejudicial attitudes. However, a few studies have shown opposite effects, with Black defendants being treated more leniently than White defendants (e.g., Poulson, 1990), and some archival analyses have shown no effect for defendant race (e.g., Pfeifer \& Ogloff, 1991; Williams \& Holcomb, 2001). In their meta-analysis, Mitchell, Haw, Pfeifer, and Meissner (2005) defined racial bias as different treatment of racial/ethnic out-group and in-group members and found a small, but significant tendency for a greater number of guilty verdicts to be assigned to out-group defendants, particularly among Black (versus White) participants and when jury instructions were not provided. However, few studies included data from Black participants, and the majority of these used continuous guilt measures — rather than dichotomous verdict choice — which were associated with stronger out-group bias. Caution is warranted when interpreting the moderating effect of participant racial/ethnic identity on juror decisions. Still, it appears that the defendant's racial/ethnic identity may affect juror decisions.

Little research has compared Indigenous and White defendants. Pfeifer and Ogloff (2003) had participants read a transcript of a sexual assault trial, in which, depending on the condition, the defendant and the victim were described as English Canadian, French Canadian, or Native Canadian, in a fully factorial design. Compared to English and French defendants, Native 
Canadian defendants were rated as more likely to be guilty when the victim was English or French. As well, the English defendant was less likely to be guilty when the victim was Native Canadian than for the other victims. Finally, ratings of the English Canadian defendant's personality traits were significantly more positive than ratings for the French Canadian or Native Canadian defendants. This likely represents in-group bias, as the sample was recruited from a mid-western university in Canada where the majority of the participants were likely English Canadians.

Particular crimes are perceived as stereotype-congruent with particular racial groups, for example, soliciting, assault-mugging, grand theft auto, and assaulting a police officer are associated with Black individuals, whereas embezzlement, child molestation, counterfeiting, fraud, and rape are associated more with White individuals (Sunnafrank \& Fontes, 1983). Gordon, Bindrim, McNicholas, and Walden (1988) found that longer sentences were recommended for Black and for White defendants who committed stereotype-consistent crimes than when they committed stereotype-inconsistent crimes. Jones and Kaplan (2003) extended this work by including a crime (vehicular manslaughter) that was not stereotypically associated with any particular racial group. Guilty verdicts were more likely than not guilty verdicts for stereotype-consistent crimes (e.g., White defendant charged with embezzlement), but guilty and not guilty verdicts were equally likely for non-stereotype-consistent crimes (e.g., Black defendant charged with embezzlement). No effects of race on verdict choice were observed for vehicular manslaughter. For punishment, Black defendants received harsher punishments than White defendants for grand theft auto, but no effects of race on punishment were observed for embezzlement or vehicular manslaughter.

For alibis specifically, Marion, Cowan, Bowling, El-Sibaey, and Burke (2011) assessed 
the effects of race (Black or White) and socio-economic status (SES; high or low) on judgments about an alibi, the strength of the evidence against the suspect, likelihood of suspect guilt, and dichotomous verdict. The materials were based on the real-life erroneous murder conviction of Danny Brown, who was exonerated by The Innocence Project (see National Registry of Exonerations, 2015 for more details). The alibi provided to participants through an audio recording and transcript were a modified version of Brown's actual alibi, edited to set the crime in Toronto rather than Toledo, $\mathrm{OH}$. The alibi described fairly mundane activities over the course of a full day. Details were changed to manipulate socio-economic status (SES) (e.g., the type of grocery store where Brown and his friend met). The same alibi was rated as more credible and the likelihood of suspect guilt was lower when the suspect was Black compared to when the suspect was White. The evidence against the suspect was rated as marginally weaker when he was a Black male than a White male. No significant effects were found for verdict choice, though this is likely because only five participants voted guilty across all four conditions (i.e., $10.6 \%$ of sample). They suggested that participants altered their judgments about the alibi to avoid appearing racist, similar to points raised in Sommers and Ellsworth's (2001) review.

Cowan, Zannella, Marion, and Burke (2015) followed up these results using a slight modification of the Marion et al. (2011) materials and found a similar pattern of results. The case against the suspect was strengthened by adding evidence to the autopsy report to elicit more guilty verdicts from participants. The alibi was rated as more truthful, the evidence against the suspect as weaker, and the probability of guilt as lower when the suspect was Black than when he was White. Suspect race did not affect ratings of alibi accuracy. The White suspect was also 2.95 times more likely to receive a guilty verdict than the Black suspect. Additionally, White participants rated the evidence against the suspect as stronger and the likelihood the suspect was 
guilty as marginally higher than did participants from other racial/ethnic groups. No significant effects were observed for SES. Again, it appears that participants are using the race information to make their judgments, and that they may be bending over backwards to avoid appearing racist. Alternately, the weaknesses in the case may have been noticed more when the suspect was Black due to differences in case information processing, as described below.

The defendant's racial or ethnic group may also influence what information mock-jurors process in a criminal trial. Sargent and Bradfield (2004) gave White community members a description of an armed robbery case where defendant race (White vs. Black), the strength of the alibi (security footage vs. no corroboration) and motivation to process the information to make a correct decision (high vs. low) were manipulated. Under high motivation, the strength of the alibi was the primary determinant of likelihood of guilt judgments; likelihood of guilt was higher when the alibi was uncorroborated than when it was corroborated by strong physical evidence. The Black defendant was rated as marginally less likely to be guilty than the White defendant, paralleling Marion et al.'s (2011) and Cowan et al.'s (2015) findings. Race and alibi strength did not interact, suggesting that the alibi information was processed similarly regardless of the defendant's racial group. In contrast, under low motivation, racial group and alibi strength interacted; the alibi strength influenced probability of guilt judgments for the Black defendant, but not for the White defendant. Although they do not report any post-hoc tests of this two-way interaction under low motivation, examination of the graph suggests that the defendant was judged significantly less likely to be guilty when he was Black and provided a strong alibi compared to any of the other conditions, which do not appear to vary.

In sum, it appears that the characteristics of the defendant, notably racial group and possibly gender, can affect legal outcomes, sometimes in complex ways. It is currently unclear 
what factors other than race salience may be responsible for determining when jurors manifest their prejudice against out-group members and when they take pains to avoid appearing racist.

Authoritarianism. In addition to the demographic characteristics of the defendant/suspect, it is also important to consider individual difference variables - their level of Authoritarianism in particular. Authoritarianism was first conceived by Adorno, FrenkelBrunswik, Levinson, and Sanford (1950) as a way to identify individuals who might be susceptible to fascist ideology. Altemeyer (1981) reframed the construct after noting that the most successful items on Adorno et al.'s (1950) scale related to aggressive impulses, endorsement of traditional social values, and submission to authority, and defined Right-Wing Authoritarianism (RWA) as the confluence of these three factors. As a result, the RWA items all measure two or three of the factors. Although it is generally accepted that these are the central traits of authoritarianism, more recently, Duckitt, Bizumac, Krauss, and Heled (2010) have treated them as separate, though related, constructs in a multidimensional conceptualization of Authoritarianism. This difference is partly based on disagreement regarding whether Authoritiarianism reflects a personality attribute (i.e., unidimensional by Altemeyer [1981]) or social attitudes and values (i.e., multidimensional related constructs by Duckitt et al. [2010]) that could reflect group cohesion or group conformity (Duckitt et al. 2010). Duckitt et al. (2010) argue that Altemeyer's (1981) RWA scale actually reflects social attitudes and values because respondents are asked to rate agreement with statements about social attitudes/beliefs, the scale is highly correlated with measures of conservative social values, the scale correlates poorly with Big Five personality dimensions, and results on the RWA can be affected by situational threat. Duckitt et al. (2010) created the Authoritarianism-Conservatism-Traditionalism (ACT) scale using rewritten items from Altemeyer's 1981 and 1998 RWA scales and items from related 
scales. Their results suggest that Authoritarianism is best viewed as a multidimensional construct.

Authoritarianism is one of the few constructs that has reliably demonstrated significant associations with legally relevant outcomes. Higher Authoritarianism is related to more guilty verdicts and convictions (Devine, Clayton, Dunford, Seying, \& Price, 2001; Narby, Cutler, \& Moran, 1993), longer recommended sentences (Devine et al., 2001), support for capital punishment (McKee \& Feather, 2008) and more skepticism of alibis (Cowan, 2012; Culhane, 2005). Using Altemeyer's RWA Scale, Cowan (2012) showed weak, but significant negative relationships between RWA and judgments of alibi strength and alibi truthfulness, and weak positive associations between Right-Wing Authoritarianism and estimations of the strength of incriminating evidence and probability of suspect guilt. It is anticipated that individuals who are higher on Authoritarianism are particularly likely to judge the character of someone providing a morally undesirable alibi harshly. A morally undesirable alibi is a violation of traditional social values (i.e., Traditionalism), and Authoritarianism is an inclination to reject and wish to punish individuals who are guilty of moral transgressions. Cowan (2012) did not find the anticipated mediating effects of perceived moral desirability on these associations, but this could be due to attenuation; none of the participants' scores met the threshold to be classified as high RWA. Thus, the influence of RWA on alibi evaluators' decisions remains unclear.

\section{Summary of Literature Review}

Alibis can be a powerful piece of evidence for the defense, as a believed alibi requires a not guilty verdict under Canadian law. However, the results of archival and empirical research suggest that true alibis may be rejected by evaluators and that, in general, alibis are viewed with skepticism. Innocent suspects may find it challenging to generate alibis due to basic memory 
processes; the mundane events that are likely to comprise an alibi narrative are unlikely to be properly encoded, large delays are possible between the events themselves and the need to recall them, and individuals' schemas may interfere with accurate recollection of the events.

The majority of the extant research on alibi believability judgments has emphasized the role that the strength of corroborative evidence (both physical evidence and alibi witness testimony) plays in the evaluation of an alibi. Physical evidence is particularly persuasive, but is frequently not available to research participants or actual suspects/defendants. In general, if an alibi witness is perceived as having a possible motive to lie, that individual's testimony will not enhance the credibility of the alibi. Additionally, there is evidence that any attempt to change an alibi, even a change that improves the quality of the corroborative evidence, may result in lower estimations of alibi believability, and that the context in which the alibi is evaluated (i.e., investigation vs. trial) and the characteristics of the alibi provider affect judgments. What is less clear is how the content of the alibi affects judgments, and whether other factors such as suspect race and the quality of the corroborative evidence interact with content.

\section{Current Dissertation}

Broadly, this dissertation investigated the conditions under which an alibi is likely to be believed or disbelieved. The two main goals of the project were to clarify the effects that the moral desirability of alibi activities may have on evaluator judgments (e.g., alibi truthfulness, probability of suspect guilt) in the context of the evaluator's social attitudes (i.e., Authoritarianism) and to begin to integrate the alibi content literature with research on other aspects of alibi believability (i.e., evidence strength, alibi provider race). A secondary goal is to investigate Cowan's (2012) finding that participants generally misunderstand the term "alibi." As the alibi believability literature is at a nascent stage, there has been a rather piecemeal approach 
to research within the field. Although progress has been made with respect to identifying and exploring the influence of a number of factors that affect alibi believability judgments (e.g., alibi provider race, quality of corroborative evidence), potentially important variables remain uninvestigated, and interrelations among these factors have yet to be explored systematically. The current project represents one of the first steps towards the goal of developing a comprehensive model of alibi believability. In the first two studies included in this dissertation, the moral desirability of alibi activities was evaluated in the context of existing research on how the strength of corroborative evidence and alibi provider characteristics (i.e., racial group) influence evaluator judgments about the alibi itself and the probability of suspect guilt. The third study clarified some of the findings from the initial two experiments.

Research questions. It was hypothesized that the content of an alibi narrative influences judgments about the alibi. As noted earlier, established social psychology research shows that humans make evaluative judgments regarding the character of others based on minimal information. In general, individuals who are perceived as having some positive traits, including physical attractiveness, are assumed to have many other positive traits, and people who are seen as having some negative traits are seen in a generally negative light. This suggests that offering a morally undesirable alibi may lead evaluators to form a negative implicit personality theory about the alibi provider and be less likely to believe the alibi. However, research on alibi moral desirability and salaciousness has shown some support for the notion that evaluators interpret the admission of bad behaviour as an indicator of honesty. In that case, a morally undesirable alibi would be more believable than a neutral alibi. Authoritarianism, however, may influence these effects, such that individuals who endorse more Authoritarian views may be likely to view an alibi provider offering a morally undesirable alibi negatively, whereas individuals who score 
lower on measures of Authoritarianism may be likely to view this same alibi as suggesting that the provider is being honest. This research sought to answer a number of important questions within the alibi believability literature. Most hypotheses are exploratory and, therefore, nondirectional. Specific questions to be addressed are:

1. Does the moral desirability of activities described in an alibi affect evaluators' (Experiments 1 and 2):

a. Judgments about the alibi (i.e., truthfulness, accuracy),

b. Judgments about the probability that the suspect is the culprit, and c. Behaviour (i.e., willingness to sign a petition in support of the suspect)?

2. Does corroboration by physical or person evidence affect alibi believability judgments? Are any effects of alibi moral desirability consistent across levels of corroboration or do some alibi narratives require more corroboration to be believable than others? (Experiment 1)

3. Are alibis provided by White and First Nations suspects viewed differently? Evidence from the analysis of criminal cases as well as experimental research suggests that members of visible minority groups are treated more harshly in their interactions within the legal system. Consequently, it is hypothesized that ratings for First Nations suspects/defendants will be less favourable (e.g., lower alibi believability, higher likelihood of guilt ratings) than for White suspects/defendants.

4. Is there an interaction between alibi moral desirability and alibi provider characteristics? For example, might a morally undesirable alibi be more believable when it is provided by a defendant/suspect who is a member of a marginalized group, and a morally desirable alibi be more believable when it is provided by a White defendant/suspect? (Experiments 2 and 3)

5. Are individuals higher in Authoritarianism more likely than those lower in Authoritarianism 
to (Experiments 1-3):

a. Provide lower ratings of alibi truthfulness and accuracy, and higher ratings of probability that the suspect is guilty, and

b. Be less likely to sign the petition?

6. Is the relationship between Authoritarianism and judgments or behaviour mediated by evaluators' perceptions of the moral desirability of the activities described in the alibi (Experiments 1 and 2)? It is expected that higher Authoritarianism will be associated with more negative views of the alibi activity, which will lead to judgments that are less favourable for the suspect.

7. Do laypersons understand what is meant by the term "alibi" (Experiments 1-3)? It is anticipated that many participants will provide definitions that are inaccurate or incomplete. 


\section{Experiment 1}

The first purpose of Experiment 1 was to investigate the influence of the moral desirability of alibi activities on legal judgments and to integrate this variable into Olson and Wells' (2004) alibi believability model. Two competing theories predicted different effects for alibi moral desirability. In the first, evaluators would develop a general implicit personality theory about the suspect and judge the alibi consistently with that theory; here, a morally undesirable alibi would be believed least and a morally desirable alibi believed most. In the second, offering a morally undesirable alibi would be interpreted as an indicator of honesty, and this alibi would be the most credible. One open question is whether any effects of alibi moral desirability are consistent across the levels of corroborative evidence strength described by Olson and Wells (2004). The second purpose of Experiment 1 was to examine the role of Authoritarianism in legal judgments, and the third purpose was to investigate participants' understanding of the meaning of an "alibi." Both of these latter two are intended to replicate Cowan's (2012) results.

Participants from Ryerson University and Iowa State University were provided with a fictional police file describing the early stages of a murder investigation. The file included a brief overview of the investigation, a suspect interrogation transcript, and a supplemental report regarding evidence that supported the alibi. The content of the suspect's alibi (morally desirable, morally undesirable, or neutral activities, plus a no-alibi control) and the strength of the evidence supporting the alibi (strong evidence, weak evidence, no evidence) were manipulated.

Participants responded to a number of questionnaire items, the Authoritarianism-ConservatismTraditionalism (ACT) scale (Duckitt et al., 2010), and provided their own definition for the term "alibi." Following this, they were asked to sign a petition supporting the suspect's request for 
assistance from Legal Aid that was ostensibly not part of the study. The study was approved by the Institutional Review Board (IRB) at Iowa State University and the Research Ethics Board (REB) at Ryerson University. The study complied with the ethical guidelines of the National Institutes of Health Office of Extramural Research (USA) and in the Tri-Council Policy Statement (Canada).

\section{Method}

Generation of materials and pilot test. The case materials in Experiment 1 were modified versions of the case files used by Cowan (2012). Three alibis were used: morally desirable, morally undesirable, and morally neutral. A group of faculty members and students first generated a list of possible alibis. To do this, a location/setting was selected and the activities that occurred there were varied.

To maximize the chance of our morally desirable, undesirable, and neutral statements being broadly perceived as such, the initial alibis generated by the group were pilot tested with a small group of colleagues and friends. These individuals rated the moral desirability of activities that took place at a friend's home (having an affair with the friend's partner; stealing items; retrieving items the friend had borrowed; helping to paint the friend's kitchen; fixing the friend's sink; helping the friends move; and babysitting the friend's children) or at a retirement home (extorting money from an elderly relative; working at a retirement home; running a scam on a non-relative senior citizen; and visiting/caring for an elderly relative with dementia). The second list did not yield a neutral statement, so the alibis were chosen from the first list. Retrieving items that had been loaned to a friend was the only neutral statement from that list, so it was chosen as the neutral alibi. Having an affair with the friend's partner was the most consistently negatively viewed statement, and assisting friends with a move was the most consistently positively viewed 
statement, so they were chosen as the morally undesirable and desirable alibis, respectively. The alibis were included in different versions of a police interrogation transcript. Security footage (strong) and a parking receipt (weak) were the only types of physical evidence that could reasonably be used in the scenario, and were the evidence types used by Olson and Wells (2004), so no pilot test was conducted for evidence strength.

Participants. Participants were recruited from the undergraduate research participation pools of Ryerson University $(N=162)$ and Iowa State University $(N=138)$. The recruitment information is provided in Appendix A. Potential participants were told that they would be reading about and evaluating details of a criminal investigation, but were not told that the focus of the study was on alibis.

Initial power calculations using G*Power 3.1.7 (Faul, Erdfelder, Lang, \& Buchner, 2007) - conducted when it was anticipated that all participants would come from Ryerson - indicated a required sample size of 196 for .80 power and an estimated two-way interaction of moral desirability and the strength of physical evidence with an effect size of .25 . The required sample size increased to 304 if the estimated interaction effect size was .20. The initial sample size was intended to be 250 participants, an average of the two estimates, but this was increased to 300 when participants from Iowa State were also recruited. Recruitment from this second site occurred out of convenience; the author visited Iowa State for a semester.

Participants at both locations were awarded one credit in their psychology courses for an hour of participation. At Ryerson, all students enrolled in the course were eligible, whereas at Iowa State, only all students who were 18 or older were eligible to participate. The difference with the age restriction is due to the variations in ethical rules and procedures between the two institutions and the relevant governing bodies. Data from six participants were excluded due to 
previous participation in alibi studies, direct experiences with the legal system, errors in study administration, or failing to complete the task as required.

Descriptive information regarding the two samples is provided in Table 1. The age distributions of both samples are notably skewed to the right and include several outliers, particularly in the Ryerson sample. No significant differences in median age between sample were observed with the Mann-Whitney $\mathrm{U}$ test, which showed a significance value of $p=.198$. The proportion of women was significantly higher in the Ryerson sample than in the Iowa State sample, $z=4.77, p<.001^{10}$. Finally, notable differences between samples can also be observed with participants' self-identified racial/ethnic group. Participants were permitted to choose more than one option, and 11 participants did so, meaning that the categories are not mutually exclusive. The sample from Iowa State was predominantly White (90.6\%), whereas the Ryerson sample was highly multicultural. At Ryerson, nearly $20 \%$ of participants chose the "other" option. The majority of participants who selected "other" identified themselves as South Asian, Southeast Asian, or Indian/West Indian, though some specified a particular country of origin (e.g., Italian, Portuguese, Chinese/Vietnamese).

Design. The study was a 3 (Moral Desirability of Alibi Activities: Undesirable, Neutral, Desirable) X 3 (Physical Evidence Strength: None, Weak, Strong) between-subjects design. A no-alibi control condition was also included. No significant effects for sample location were hypothesized, but Location was included as a between-subjects variable in all relevant analyses.

Materials. The police file that participants read, which described the initial stages of a murder investigation, was modified from Cowan (2012) (see Appendix E), and included three documents: a Summary Report, an Interrogation Transcript, and a Supplemental Evidence

\footnotetext{
${ }^{10}$ Calculated using VassarStats' computation for the significance of the difference between two independent proportions.
} 
Report. The file included details of a murder investigation a that ostensibly took place in Seattle, WA, and a transcript of a suspect interrogation. The Summary Report included basic information about the case, including details of the 911 call, the crime scene, a statement from a witness who saw the perpetrator flee the scene, and a follow-up note dated two months after the crime regarding an anonymous tip that identified the suspect. Summary reports were identical across conditions.

Table 1

Participant demographic information

\begin{tabular}{lccc}
\hline & Ryerson & Iowa & Combined \\
\hline Age & & & \\
Mean & 20.73 & 19.38 & 20.11 \\
Standard Deviation & 5.81 & 1.64 & 4.44 \\
Median & 18.00 & 19.00 & 19.00 \\
Median Absolute Deviation & 1.00 & 0.00 & 1.00 \\
$\quad$ Range & $17-50$ & $18-32$ & $17-50$ \\
Gender & & & \\
Women & $132(81.5 \%)$ & $78(56.5 \%)$ & $210(70 \%)$ \\
Men & $30(18.5 \%)$ & $60(43.5 \%)$ & $90(30 \%)$ \\
Racial/Ethnic Group & $13(8.0 \%)$ & $4(2.9 \%)$ & $17(5.7 \%)$ \\
Black or African American & $46(28.4 \%)$ & $3(2.2 \%)$ & $49(16.3 \%)$ \\
East Asian & $4(2.5 \%)$ & $4(2.9 \%)$ & $8(2.7 \%)$ \\
Hispanic or Latino/a & $17(10.5 \%)$ & $3(2.2 \%)$ & $20(6.7 \%)$ \\
West Asian/Middle Eastern & $59(36.4 \%)$ & $125(90.6 \%)$ & $184(61.3 \%)$ \\
White & $1(0.6 \%)$ & $0(0 \%)$ & $1(0.3 \%)$ \\
Native Hawaiian or Other Pacific & & & \\
$\quad$ Islander & $32(19.8 \%)$ & $1(0.7 \%)$ & $33(11.0 \%)$ \\
Other &
\end{tabular}

Note. Seven participants ( 5 from Ryerson and 2 from Iowa) did not provide their age. One participant at Ryerson identified as both male and female, and another did not respond.

Transcripts of genuine police interrogation available online were used as guides to increase the ecological validity of the Interrogation Transcript used in the experiment, particularly with the wording of the interrogator's questions. In the transcript, the suspect indicated that he was either (a) having an affair with his friend's romantic partner (morally 
undesirable alibi), (b) helping the friend's romantic partner to move (morally desirable alibi), (c) retrieving items from the friend's romantic partner that he had loaned to them (neutral alibi), or (d) unable to remember his whereabouts (no-alibi control). In all alibi conditions, the friend was absent. The word counts across conditions were kept as similar as possible to ensure that the amount of information participants received would not confound the results. When an alibi was presented, a brief Supplemental Evidence Report was included in the file that indicated either that (a) video footage from a parking lot security camera corroborated the suspect's alibi (strong evidence), (b) there was no video evidence because the security camera was broken, but the suspect provided a receipt from the parking lot to corroborate his alibi (weak evidence), or (c) or that there was no security footage to confirm the alibi and the suspect had lost the parking lot receipt (no evidence). In all three versions, the suspect provided the name of his friend's partner, who could corroborate his alibi. The quality of the physical evidence is the key evidence manipulation in the study because, when present, it has been shown to neutralize the influence of person evidence quality (e.g., Olson \& Wells, 2004). As participants in the control condition were not given information about an alibi, the supplemental evidence to corroborate the alibi was not included in the file for participants in this condition.

Data were obtained through a questionnaire administered online through qualtrics.com (see Appendix F). The questionnaire had five main sections: demographic information, judgments about the case, non-legal judgments, manipulation checks, and a measure of Authoritarianism. The legal judgments were of primary interest, so they were presented first to ensure that responses were unaffected by order effects. The manipulation checks were included later to ensure that participants did not intuit the study's objectives and change their responses. Authoritarianism was not expected to be influenced by the materials or earlier questionnaire 
items. The demographic questions included items regarding gender, age, and race/ethnicity. For judgments about the case, all participants rated their perceptions of the honesty of the suspect's description of his whereabouts during the crime on a scale from 1 (Not at all honest) to 7 (Very honest) and their confidence in that judgment on a scale from 1 (Not at all confident) to 7 (Very confident). Participants who were shown an alibi then rated the accuracy of the suspect's statement about his whereabouts on a scale from 1 (Not at all accurate) to 7 (Very accurate), and their confidence in that judgment, as above. These items were not applicable for the control condition and were not shown to control participants. Participants who saw an alibi then classified it as true ("The truth; he was telling police where he was at the time"), fabricated ("A lie; he was deliberately giving false information to the police"), or mistaken ("An error; he was trying to tell the truth, but was mistaken about his whereabouts"), in keeping with Burke et al.'s (2007) categories. All participants then rated the strength of the evidence against the suspect on a scale from 1 (Not at all strong) to 7 (Very strong), the likelihood that the suspect is guilty on a scale from 1 (Not at all likely) to 7 (Very likely), and provided a verdict (Guilty or Not Guilty). For non-legal judgments, all participants rated how similar they were to the suspect in terms of personality on a scale from 1 (Not at all similar) to 7 (Very similar), how much they liked the suspect on a scale from 1 (Not at all) to 7 (A lot), and what they thought of the suspect as a person from 1 (A very bad person) to 11 (A very good person).

Manipulation check questions were also included. An open-ended question asked all participants to report what the suspect said he was doing at the time of the crime. Participants who read an alibi were then asked whether they themselves had ever engaged in the behaviour described in the alibi (Yes or No); participants who had engaged in the behaviours might perceive them differently than participants who had not. Participants described the evidence to 
support the suspect's alibi in an open-ended question. They also evaluated the behaviour described in the alibi and provided their impressions of an average adult male in North America who engaged in this behaviour on a scale from 1 (Very Negative) to 11 (Very Positive). All participants were asked to define the term "alibi," provide any general comments about the case in two open-ended items. In the final section of the questionnaire, participants completed the short form of Duckitt et al.'s (2010) Authoritarianism-Conservatism-Traditionalism Scale (see Appendix G). This scale includes six items for each of the three subscales, half of which are reverse-scored. Participants rate their agreement on a scale from 1 (Strongly Disagree) to 7 (Strongly Agree).

After they completed the questionnaire, participants were shown a fictitious petition, created at www.importantpetitions.com, modelled after websites like change.org and care2actionalerts.com (see Appendix H). The petition included a photograph of a judge's gavel and a set of scales; a brief description of a campaign on behalf of the suspect to request assistance from Legal Aid Washington; and entry fields for a signatory's name, city, province or state, postal or ZIP code, an optional text-entry box, and a large button to submit a signature. These fields are commonly collected for genuine petitions. To maintain participants' privacy as much as possible, only the information entered in the name fields was saved.

The particular photo was chosen because a gavel and set of scales are both widely known symbols for justice and the legal system. Ordinarily, petition websites include a photo of the individual(s) who are the subject of the petition. An object was chosen because perceptions of the individual in the photo could influence participants' willingness to sign the petition. As well, a photograph of objects would allow the petition to be used in subsequent studies in which the demographic characteristics of the suspect were manipulated. 
Procedure. Participants, in groups of 1-4, were invited into a testing room either in the Psychology Research and Training Centre (PRTC) at Ryerson University or the Applied Cognition Laboratory at Iowa State University. In the former, participants were seated at different stations in a single room. In the latter, participants were given informed consent and debriefed in a group, but completed all experimental tasks in small individual rooms within the lab. During informed consent, participants were told that they would be reading about a criminal investigation and would be asked to answer questions about their impressions of the case and complete a personality measure.

After informed consent was obtained, participants were given the police file, and the researcher left the room. The version participants read was randomly assigned. When participants finished reading the file (approximately 10-15 minutes) the documents were removed and participants completed all sections of the questionnaire individually on a PC computer. Finally, the researcher presented the petition, explaining that it was something the research team had found online and thought that participants might be interested in looking at, given that they had read some of the details of the case. Participants were told that signing the petition was optional. To ensure that participants did not feel undue pressure to sign the petition, the researcher told the participants to close the computer window when they were finished with the petition - regardless of whether they had signed it - and left the room for several minutes. This was under the guise of needing to retrieve debriefing forms from the lab office or, at Iowa State, needing to check on other participants.

After participants had been given several minutes with the petition, the researcher debriefed participants and revealed the purpose of the petition. The researcher then asked the participants whether they had believed that the petition was genuine, or if they had been 
suspicious. During debriefing, 18 participants indicated high levels of suspicion about the petition. They were excluded from analyses related to the petition, but their questionnaires were retained. Participants were given a re-consent form (see Appendices C and D). The initial form included a brief statement that participants were agreeing to the use of the data and a signature line. The form was later changed to a checkbox format, because the initial form required participants who wished to refuse consent do so verbally and explicitly, and this could cause embarrassment or discomfort. Participants were asked to consent to the recording of their name and participant number to track petition-signing with one set of checkboxes and to consent to use of their questionnaires in another set. All participants at Iowa State consented to all procedures. A number of participants at Ryerson consented to use of the questionnaires, but not to have their names recorded.

\section{Results}

Manipulation checks. Tests of the manipulations were computed for participants who were not in the control condition (details are available in Appendix I). As noted earlier, it was important that participants viewed the morally desirable, undesirable, and neutral alibis as such. This was evaluated by using participants' ratings of how positively or negatively they viewed the alibi activity, as well as their impressions of an individual who engaged in that activity (i.e., the actor). First, significant differences in these ratings should be observed between all alibi conditions, and second, ratings for the Morally Undesirable and Desirable conditions should be significantly different from the midpoint of the scale, but ratings for Neutral should not.

Significant differences were found for ratings of moral desirability of the action and actor between all alibis at both sample sites. As well, results indicated that the behaviour described in the morally undesirable alibi was rated as negative (i.e., significantly below the midpoint), the 
morally desirable alibi behaviour was rated as positive, and the neutral alibi behaviour was rated as neutral. For ratings of the actor, the data from Iowa State indicated that all actors were perceived as expected, but at Ryerson, the actor in the Morally Undesirable condition was rated negatively, but the actors in the Morally Desirable and Neutral alibis were both rated as neutral. Still, in general, the manipulations operated as intended. It is worth noting that participants reported being much less likely to have engaged in the Morally Undesirable activity (5.7\%) than in the Neutral (60.0\%) or Morally Desirable activities $(67.4 \%)$.

Aspects of the alibi. The first set of analyses explored the effect of aspects of the alibi and followed up on differences between the samples from Iowa State and Ryerson.

Alibi versus no-alibi control. In the Interrogation Transcript, participants in the Alibi conditions read about a specific alibi, whereas those in the No-Alibi Control condition read about the suspect's typical Sunday evening (i.e., when the murder occurred). To assess whether having an alibi, regardless of its content, affected legal judgments relative to the absence of an alibi, 2 (Alibi vs. No-Alibi Control) X 2 (Location: Ryerson vs. Iowa State) analyses were computed on all dependent variables. Location was included to establish whether the results were consistent across both sites. The majority of these analyses are univariate factorial ANOVAs.

Suspect honesty. Participants rated how honest the suspect had been during the interrogation (see descriptive statistics in Table 2). Significant main effects showed that the statement was judged as being more honest when an alibi was provided than when there was no alibi, $F(1,296)=29.061, p<.001, \eta_{\mathrm{p}}^{2}=.089$, and by the Iowa State sample compared to the Ryerson sample, $F(1,296)=12.54, p<.001, \eta_{\mathrm{p}}^{2}=.041$. These effects were qualified by a significant interaction, $F(1,296)=8.61, p=.034, \eta_{\mathrm{p}}^{2}=.015$. Independent-samples $t$-tests were 
computed using a Holm-Bonferroni correction ${ }^{11}$ to control the familywise error rate; this correction was applied throughout the dissertation whenever there were multiple comparisons involving the same cells. Reported $p$-values were not adjusted. Results indicated that ratings of perceived honesty were higher when an alibi was provided than when there was no alibi in both samples, but the difference between the two was larger at Ryerson, $t(160)=5.17, p<.001, d=$ 1.36 , than at Iowa State, $t(136)=2.38, p=.019, d=.62$.

Table 2

Descriptive statistics for comparison of alibi vs. no-alibi control conditions by sample location

\begin{tabular}{llcc}
\hline & & $\begin{array}{c}\text { Iowa State } \\
M(S D)\end{array}$ & $\begin{array}{c}\text { Ryerson } \\
M(S D)\end{array}$ \\
\hline Suspect honesty & Alibi & $4.77(1.32)$ & $4.41(1.42)$ \\
& No-Alibi Control & $3.94(1.48)$ & $2.50(1.27)$ \\
Confidence in suspect honesty & & $4.88(1.37)$ & $4.78(1.39)$ \\
& Alibi & $5.18(1.43)$ & $4.63(1.89)$ \\
Strength of evidence against suspect & No-Alibi Control & & \\
& & $2.88(1.29)$ & $3.53(1.55)$ \\
& Alibi & $3.82(1.55)$ & $3.69(1.62)$ \\
Likelihood of guilt & No-Alibi Control & $3.29(1.32)$ & $3.84(1.50)$ \\
& & $4.00(1.54)$ & $4.31(0.95)$ \\
\hline & Alibi & No-Alibi Control & \\
\hline
\end{tabular}

Note. Although statistical analyses for estimates of the strength of the evidence against the suspect were computed on data that had been subjected to a square root transformation, to maximize the interpretability of the results, non-transformed descriptive statistics are reported.

Confidence in ratings of suspect honesty. Participants also rated their confidence in their evaluation of the honesty of the suspect's statement. The main effects for Alibi/No-Alibi Control, $F(1,296)=.068, \mathrm{p}=.794, \eta_{\mathrm{p}}^{2}<.001$, and Location, $F(1,296)=1.58, p=.210, \eta_{\mathrm{p}}^{2}=$ .005 , were not statistically significant. The interaction between the two also failed to reach

\footnotetext{
${ }^{11}$ In this procedure, the alpha criterion is determined by the number of comparisons remaining. To illustrate, if there are a total of three pairwise comparisons, the first alpha criterion is set at $.017(.05 / 3)$. If the null hypothesis is rejected in this comparison, the next alpha criterion is set at .025, because there are two comparisons remaining. An important note is that if the null hypothesis is not rejected in a comparison, the procedure ends and any remaining comparisons are not compared to alpha.
} 
significance, $F(1,296)=.74, p=.391, \eta_{\mathrm{p}}^{2}=.002$.

Strength of the evidence against the suspect. For this analysis, significantly greater variance was observed in the condition with the smaller sample size. A square root transformation equalized the variance and data remained normal. The evidence against the suspect was judged as being marginally weaker when he provided an alibi compared to when there was no alibi, $F(1,295)=3.73, p=.054, \eta_{\mathrm{p}}^{2}=.012($ see Table 2$)$. Neither the main effect for Location, $F(1,295)=.75, p=.386, \eta_{\mathrm{p}}^{2}=.003$, nor the interaction between the two variables, $F(1$, $295)=2.16, p=.143, \eta_{\mathrm{p}}^{2}=.007$, were significant.

Likelihood of guilt. Participants were asked to indicate how likely they thought it was that the suspect was guilty. Participants in the Alibi conditions rated the likelihood of suspect guilt as significantly lower than participants in the Control condition, $F(1,296)=5.16, p=.025, \eta_{\mathrm{p}}^{2}=$ .017 . The main effect for Location, $F(1,296)=2.78, p=.097, \eta_{\mathrm{p}}^{2}=.009$, and the interaction term, $F(1,296)=.22, p=.644, \eta_{\mathrm{p}}^{2}=.001$, did not achieve significance.

Verdict choice. A loglinear analysis using backward elimination was computed with Sample Location, Alibi/No-Alibi Control, and Verdict Choice. Results indicated good model fit, $\chi^{2}(3)=3.96, p=.398$. Only the Alibi/No-Alibi Control main effect-which is due simply to differences in sample sizes - and the Location X Verdict Choice interaction remained in the model. Overall, $28.3 \%$ of participants provided guilty verdicts. More guilty verdicts were provided by the participants at Ryerson (33.3\%) than by the participants at Iowa State (22.5\%). Alibi/No-Alibi Control was not reliably associated with verdict choice, though participants in the Alibi condition $(n=267)$ provided guilty verdicts $27.0 \%$ of the time, and participants in the NoAlibi Control condition $(n=33)$ provided guilty verdicts $39.4 \%$ of the time.

Petition signing. Following completion of the questionnaire, participants were shown a 
petition in support of the suspect's request for assistance from Legal Aid Washington. Overall, $52.8 \%$ of participants signed the petition ( $N=254$; see Table 3 for descriptive statistics). A loglinear analysis with backward elimination was computed on Location, Alibi/No-Alibi Control, and petition-signing. The model was a good fit for the data, $\chi^{2}(6)=5.90, p=.434$. However, the final model included only the Alibi/No-Alibi Control effect. Again, this is due to the greater number of participants assigned to the alibi conditions than the control condition. Verdict choice was not affected by Alibi/No-Alibi Control or Location; the apparently higher percentage for the Iowa State Control cell represents only 13 participants, so is unlikely to be reliable (see Table 3).

Table 3

Rates of petition-signing at Iowa State and Ryerson

\begin{tabular}{lcc}
\hline & Iowa State & Ryerson \\
\hline Alibi & $55.0 \%$ & $52.9 \%$ \\
No-Alibi Control & $47.8 \%$ & $76.9 \%$ \\
\hline
\end{tabular}

Note. The cell representing Ryerson participants in the No-Alibi Control includes only 13 data points and the cell representing Iowa State participants comprises only 17 data points.

Comparisons of ratings of the different alibis and the no-alibi control. One of the primary research questions of this work is to investigate under which conditions having an alibi is helpful, compared to when a suspect does not have an alibi. Of particular interest here is the comparison between the control condition and each of the various alibi conditions. For each dependent measure, an initial omnibus test (either one-way ANOVA or chi-square) was computed comparing responses for the Morally Desirable, Morally Undesirable, Morally Neutral, and No-Alibi Control conditions (see Table 4 for descriptive statistics of continuous dependent measures). Planned comparisons ( $t$-tests or chi-square tests) were then computed comparing each of the alibi conditions to the control, regardless of whether the ANOVA was 
significant. Too few participants were in the control condition at Ryerson and at Iowa State to allow for results to be computed separately by site.

Table 4

Means and standard deviations for alibi and control conditions

\begin{tabular}{lcccc}
\hline & Morally & Morally & Morally & Control \\
& Desirable & Neutral & Undesirable & \\
& $M(S D)$ & $M(S D)$ & $M(S D)$ & $M(S D)$ \\
\hline Suspect honesty & $* 4.63(1.23)$ & $* 4.55(1.42)$ & $* 4.54(1.50)$ & $3.24(1.54)$ \\
$\begin{array}{c}\text { Confidence in suspect } \\
\text { honesty }\end{array}$ & $4.84(1.37)$ & $4.85(1.33$ & $4.79(1.44)$ & $4.91(1.67)$ \\
$\begin{array}{c}\text { Strength of the evidence } \\
\text { against the suspect }\end{array}$ & $\wedge 3.06(1.61)$ & $3.36(1.38)$ & $3.31(1.42)$ & $3.76(1.56)$ \\
Likelihood of suspect guilt & $* 3.40(1.52)$ & $3.69(1.38)$ & $3.68(1.43)$ & $4.15(1.28)$ \\
\hline
\end{tabular}

Note. Alibi ratings denoted with a $*$ differed significantly from the rating of the Control group. Alibi ratings denoted with $\mathrm{a}^{\wedge}$ approached significance.

Suspect honesty. Participants' ratings of the honesty of the suspect's statement were significantly affected by the alibi condition, $F(3,296)=8.84, p<.001, \eta_{\mathrm{p}}^{2}=.082$. Follow-up analyses revealed that ratings for statement honesty were significantly lower for the No-Alibi Control than for the Morally Desirable, $t(120)=5.16, p<.001, d=1.05$, Morally Undesirable, $t(118)=4.20, p<.001, d=.86$, or Neutral conditions, $t(122)=4.42, p<.001, d=.90$.

Confidence in suspect honesty. Alibi condition did not affect participants' self-reported confidence in their ratings of the suspect's honesty, $F(3,296)=.06, p=.982, \eta_{\mathrm{p}}^{2}=.001$. No significant differences emerged when the Control condition was compared to the Morally Desirable, $t(120)=-.22, p=.823, d=-.05$, the Morally Undesirable, $t(118)=-.38, p=.707, d=-$ .08 , or Neutral conditions, $t(122)=-.22, p=.829, d=-.04$.

Strength of the evidence against the suspect. Alibi condition did not affect ratings of the strength of the evidence against the suspect, $F(3,295)=1.90, p=.131, \eta_{\mathrm{p}}^{2}=.019$. In the context of three comparisons, there was a trend towards lower ratings of the strength of the evidence for 
the Morally Desirable condition than the Control condition, $t(120)=-2.15, p=.033, d=-.44$. No significant differences were found between the Control and the Morally Undesirable, $t(118)=$ $1.50, p=.136, d=-.31$, or Neutral conditions, $t(121)=-1.38, p=.169, d=-.28$.

Likelihood of suspect guilt. Ratings of the likelihood that the suspect was guilty did not vary depending on alibi condition, $F(3,296)=2.28, p=.080, \eta_{\mathrm{p}}^{2}=.023$. One significant planned comparison was observed; the likelihood of suspect guilt was rated as significantly lower when the suspect offered a morally desirable alibi than when he did not offer an alibi, $t(120)=-2.51, p$ $=.013, d=-.51$. Ratings for the Control condition were not significantly different than ratings in the Morally Undesirable, $t(118)=-1.67, p=.098, d=-.34$, or Morally Neutral, $t(122)=-1.67, p$ $=.098, d=-.34$, conditions.

Verdict choice. The overall rate of guilty verdicts for the entire sample was $28.3 \%$. Alibi condition, overall, was unrelated to verdict choice, $\chi^{2}(3)=2.59, p=.464, V=.093$. In the planned comparisons, the rate of guilty verdicts in the Control condition (39.4\%) did not differ significantly from the rates of guilty verdicts in the Morally Desirable $(24.7 \%), \chi^{2}(1)=2.53, p=$ $.121, \varphi=-.14$, Morally Undesirable $(27.6 \%), \chi^{2}(1)=1.56, p=.269, \varphi=-.11$, or Neutral conditions $(28.6 \%), \chi^{2}(1)=1.32, p=.278, \varphi=-.10$.

Participants' willingness to sign the petition. The overall rate of petition-signing was $52.8 \%$. The omnibus test of the effect of alibi condition on willingness to sign the petition was not significant, $\chi^{2}(3)=3.43, p=.335, V=.12$. In the planned comparisons, rates of petitionsigning were equivalent in the Control condition (63.3\%) and in the Morally Desirable (57.1\%), $\chi^{2}(1)=.34, p=.663, \varphi=-.06$, Morally Undesirable $(50.7 \%), \chi^{2}(1)=1.36, p=.280, \varphi=-.12$, or Neutral conditions $(46.1 \%), \chi^{2}(1)=2.57, p=.133, \varphi=-.16$.

Overall, the results comparing the responses of participants who read about an alibi and 
those who did not showed that alibis benefit suspects in some instances. A Morally Desirable alibi appeared to be particularly likely to lead to ratings that were beneficial for the suspect.

The effect of alibi content, corroborative evidence, and sample location. To examine the effects of alibi content, corroborative evidence, and sample location, a series of 3 (Alibi Moral Desirability: Morally Desirable, Morally Undesirable, Neutral) X 3 (Evidence Strength: Strong, Weak, None) X 2 (Location: Iowa State, Ryerson) were computed on the dependent measures. Where figures are presented, Masson and Loftus' (2005) between-subjects error bars were used.

Suspect honesty. For ratings of the honesty of the suspect, the only significant effects are main effects for Evidence Strength and Location (see Table 5 for statistical results). As discussed earlier, ratings of suspect honesty were higher at Iowa State than at Ryerson. In the context of three comparisons, only one of the pairwise comparisons was significant; the suspect's statement was rated as significantly more honest when there was strong physical evidence to support it than when there was no physical evidence, $t(179)=3.80, p<.001, d=.57$. The suspect's statement was rated as equally honest between the Strong and Weak, $t(178)=2.10, p=.037, d=.31$, and Weak and No Evidence conditions, $t(171)=1.74, p=.084, d=.27$ (see Figure 1). Although the $p$-value for the comparison between Strong and Weak was less than .05 , it is not significant with the Holm-Bonferroni correction, as the alpha level in this case was .025 (i.e., .05 divided by the two comparisons that remained after the Strong versus None comparison). Essentially, only relatively strong evidence supporting an alibi was able to increase estimates of suspect honesty significantly compared to when such evidence was lacking.

Confidence in ratings of suspect honesty. No significant effects were found for participants' confidence in their rating of suspect honesty (see Table 6). 
Table 5

ANOVA results for participants' ratings of suspect honesty

\begin{tabular}{lcccc}
\hline Effect & $d f$ & $F$ & $p$ & $\eta_{\mathrm{p}}^{2}$ \\
\hline Alibi Content & 2,249 & .19 & .825 & .002 \\
Evidence Strength & 2,249 & 6.62 & .002 & .050 \\
Location & 1,249 & 4.13 & .043 & .016 \\
Alibi Content X Evidence Strength & 4,249 & .31 & .871 & .005 \\
Alibi Content X Location & 2,249 & .75 & .474 & .006 \\
Evidence Strength X Location & 2,249 & 1.02 & .361 & .008 \\
Alibi Content X Evidence Strength X Location & 4,249 & 1.56 & .185 & .024 \\
\hline
\end{tabular}

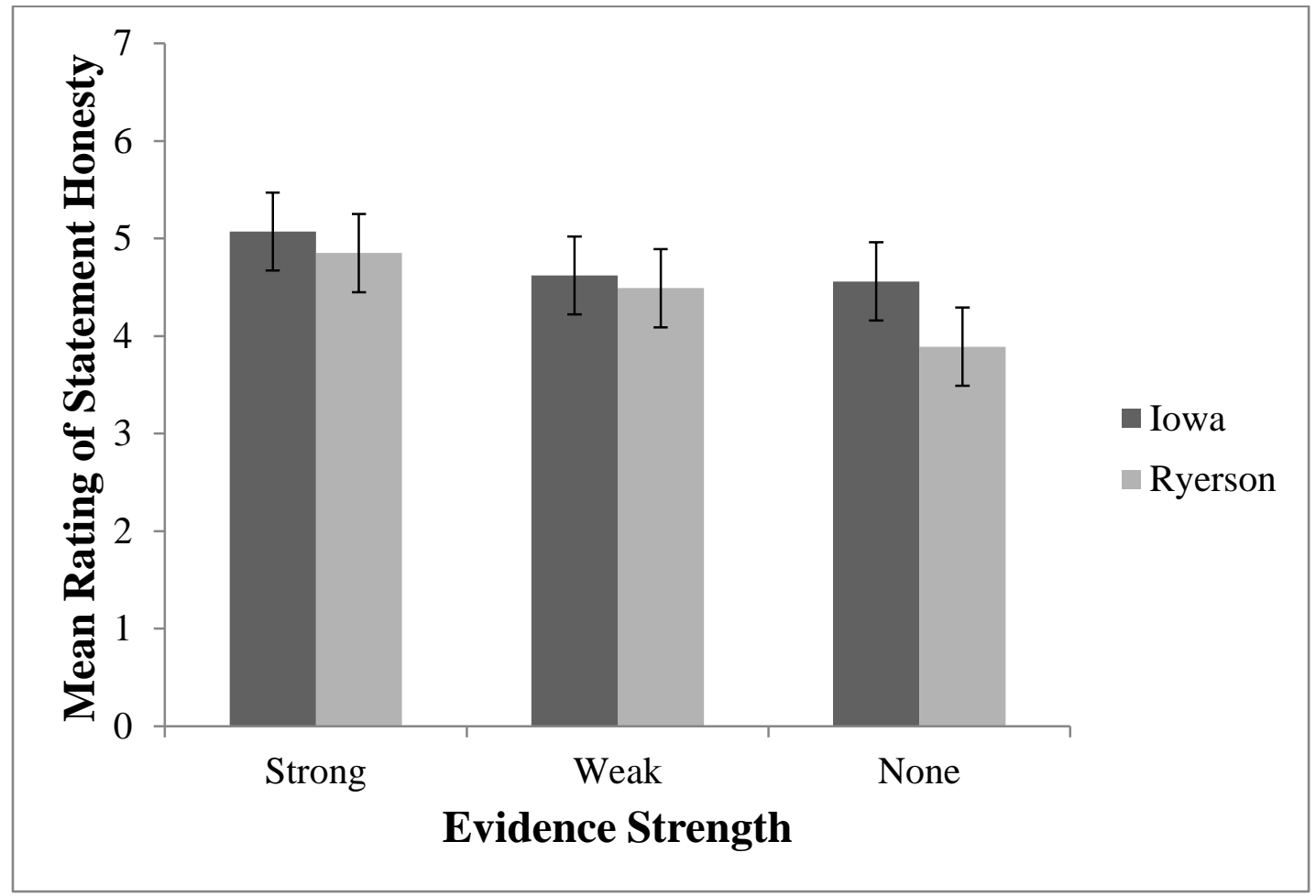

Figure 1. Mean ratings of statement honesty for different levels of alibi corroboration at Ryerson and Iowa State.

Alibi accuracy. For participants' ratings of the accuracy of the suspect's description of his activities during the crime, the only significant effect was a main effect for Evidence Strength (see Table 7 for statistical results). In the context of three comparisons, the differences between the Strong $(M=5.02, S D=1.36)$ and No Evidence $(M=3.98, S D=1.55)$ conditions, $t(177)=$ 
$4.80, p<.001, d=.69$, and between the Weak $(M=4.64, S D=1.36)$ and No Evidence

conditions, $t(169)=2.97, p=.003, d=.45$, were significant, and difference between the Strong and Weak conditions was not, $t(178)=1.88, p=.062, d=.27$. The statement was rated as more accurate when there was physical evidence to support it, compared to when there was none, but the quality of the extant physical evidence was irrelevant.

Table 6

ANOVA results for participants' confidence in their ratings of suspect honesty

\begin{tabular}{lcccc}
\hline Effect & $d f$ & $F$ & $p$ & $\eta_{\mathrm{p}}^{2}$ \\
\hline Alibi Content & 2,249 & .02 & .976 & $<.001$ \\
Evidence Strength & 2,249 & 1.55 & .214 & .012 \\
Location & 1,249 & .25 & .619 & .001 \\
Alibi Content X Evidence Strength & 4,249 & .65 & .628 & .010 \\
Alibi Content X Location & 2,249 & .13 & .881 & .001 \\
Evidence Strength X Location & 2,249 & .56 & .570 & .005 \\
Alibi Content X Evidence Strength X Location & 4,249 & 1.25 & .291 & .020 \\
\hline
\end{tabular}

Table 7

ANOVA results for participants' ratings of alibi accuracy

\begin{tabular}{lcccc}
\hline Effect & $d f$ & $F$ & $p$ & $\eta_{\mathrm{p}}^{2}$ \\
\hline Alibi Content & 2,247 & .44 & .643 & .004 \\
Evidence Strength & 2,247 & 11.02 & $<.001$ & .082 \\
Location & 1,247 & .25 & .620 & .001 \\
Alibi Content X Evidence Strength & 4,247 & .84 & .502 & .013 \\
Alibi Content X Location & 2,247 & .60 & .549 & .005 \\
Evidence Strength X Location & 2,247 & 1.95 & .144 & .016 \\
Alibi Content X Evidence Strength X Location & 4,247 & 1.53 & .195 & .024 \\
\hline
\end{tabular}

Confidence in ratings of alibi accuracy. As with the previous confidence rating, no significant main or interactive effects were observed for confidence in ratings of alibi accuracy (see Table 8). 
Table 8

ANOVA results for participants' confidence in their ratings of alibi accuracy

\begin{tabular}{lcccc}
\hline Effect & $d f$ & $F$ & $p$ & $\eta_{\mathrm{p}}^{2}$ \\
\hline Alibi Content & 2,247 & .69 & .504 & .006 \\
Evidence Strength & 2,247 & .89 & .411 & .007 \\
Location & 1,247 & .13 & .717 & .001 \\
Alibi Content X Evidence Strength & 4,247 & .18 & .947 & .003 \\
Alibi Content X Location & 2,247 & 1.58 & .209 & .013 \\
Evidence Strength X Location & 2,247 & .40 & .673 & .003 \\
Alibi Content X Evidence Strength X Location & 4,247 & .45 & .770 & .007 \\
\hline
\end{tabular}

Strength of the evidence against the suspect. For participants' ratings of the strength of the evidence against the suspect, the only significant effect was the main effect for Location, with higher ratings found at Ryerson than at Iowa State (see Table 9 and Figure 2). Additionally, the interaction between Alibi Moral Desirability and Location approached significance(see Figure 2). At Ryerson, Alibi Moral Desirability did not have a significant effect, $F(1,117)=$ $4.05, p=.020, \eta_{\mathrm{p}}^{2}=.07$. At Iowa State, a main effect was observed for Alibi Moral Desirability, $F(1,143)=.19, p=.831, \eta_{\mathrm{p}}^{2}<.01$. In the context of three comparisons, the only significant difference was that the evidence against the suspect was rated as weaker in the Morally Desirable condition than in the Neutral condition, $t(78)=2.71, p=.008, d=0.61$. No significant differences were found for the Morally Undesirable condition between either the Morally Desirable, $t(79)=1.79, p=.077, d=0.40$, or the Neutral conditions, $t(77)=-1.09, p=.277, d=-$ 0.25 . However, care should be taken in the interpretation of this result, given that the interaction term did not reach traditional significance levels. 
Table 9

ANOVA results for participants' ratings of the strength of the evidence against the suspect

\begin{tabular}{lcccc}
\hline Effect & $d f$ & $F$ & $p$ & $\eta_{\mathrm{p}}^{2}$ \\
\hline Alibi Content & 2,248 & 1.56 & .212 & .012 \\
Evidence Strength & 2,248 & 2.31 & .102 & .018 \\
Location & 1,248 & 12.26 & .001 & .047 \\
Alibi Content X Evidence Strength & 4,248 & .28 & .893 & .004 \\
Alibi Content X Location & 2,248 & 2.59 & .077 & .020 \\
Evidence Strength X Location & 2,248 & .23 & .795 & .002 \\
Alibi Content X Evidence Strength X Location & 4,248 & 1.85 & .120 & .029 \\
\hline
\end{tabular}

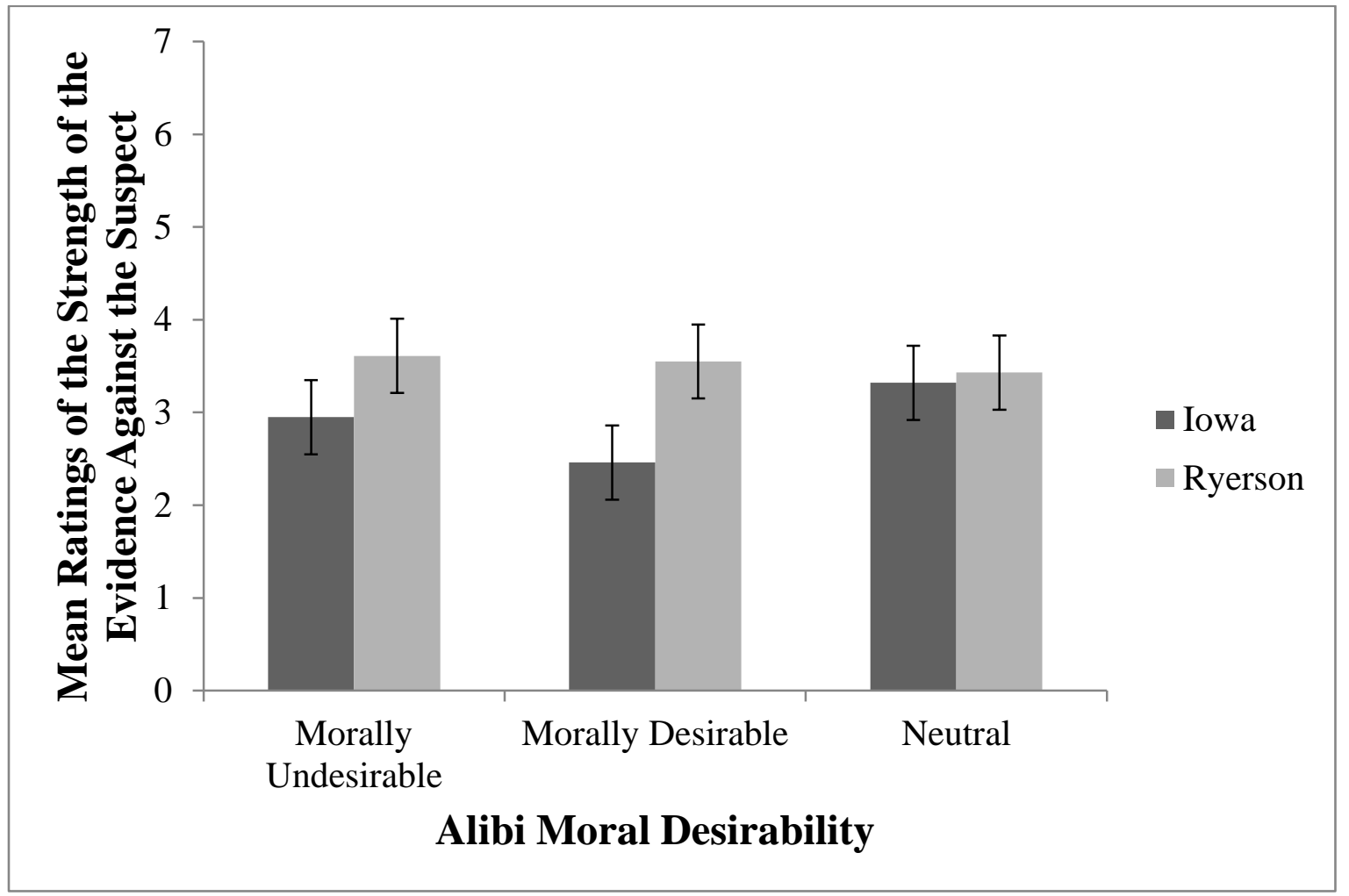

Figure 2. Mean ratings of the strength of the evidence against the suspect for different alibis, at Ryerson and Iowa State.

Alibi classification. Participants whose police files included an alibi were also asked to classify this statement as either true (i.e., honestly offered and objectively accurate), fabricated (i.e., inaccurate due to deliberate deception) or mistaken (i.e., honestly offered, but inaccurate due to an error). In both samples, the majority of participants reported that the suspect's 
statement was true and few reported that it was mistaken (see Table 10).

Table 10

Participants' classifications of the suspect's alibi as percentages

\begin{tabular}{lccc}
\hline & Iowa State & Ryerson & Total \\
\hline True & $66.1 \%$ & $52.7 \%$ & $58.8 \%$ \\
Fabricated & $21.5 \%$ & $31.5 \%$ & $27.0 \%$ \\
Mistaken & $12.4 \%$ & $15.8 \%$ & $14.2 \%$ \\
\hline
\end{tabular}

To examine whether Alibi Moral Desirability, Evidence Strength, and Location influenced participants' classification of the alibi, a multinomial logistic regression was computed. The results of the analysis showed that when an alibi was supported by physical evidence of some kind, participants were more inclined to report believing that the alibi was true rather than fabricated, compared to when the alibi was not supported by physical evidence. However, physical evidence did not affect whether participants reported that an alibi is mistaken, compared to fabricated. Overall, no clear differences were observed in classification rates between the two samples. Statistical details are provided below.

The No Evidence and Morally Neutral conditions were chosen as the reference categories, as both represent the absence of the independent variable in question. For the dependent measure, Fabricated was chosen as the reference category in the analysis; the comparison between True and Mistaken was not of primary theoretical interest, as both involve honestly-offered statements. Significant Pearson, $\chi^{2}(24)=47.72, p=.003$, and deviance, $\chi^{2}(24)=$ $56.85, p<.001$, results suggested that the model was not a good fit for the data-likely due to four cells having zero frequencies - though adding the predictors does significantly improve the model over the intercept-only model, $\chi^{2}(10)=19.39, p=.036$. Overall, adding Evidence Strength contributed significantly to the model, $\chi^{2}(4)=12.33, p=.015$. Adding Alibi Moral 
Desirability, $\chi^{2}(4)=2.27, p=.686$, and Location, $\chi^{2}(2)=4.52, p=.104$, did not.

For the logit in which the True and Fabricated categories were compared, participants were significantly more likely to report that the alibi was True (versus Fabricated) when there was strong evidence, $b=1.25, S E=.37$, Wald $=11.45, p=.001$, Odds Ratio $=3.48$, and when there was weak evidence, $b=.704, S E=.35$, Wald $=4.06, p=.044$, Odds Ratio $=2.02$, compared to when there was no evidence. Essentially, any evidence was better than no evidence. Interestingly, in this logit, participants from Iowa State were more likely to indicate that the alibi was true (rather than fabricated) than were participants from Ryerson, $b=59, S E=.30$, Wald $=$ $3.83, p=.050$, Odds Ratio $=1.80$, though the effect of Location was not significant in the overall model.

In contrast, for the logit in which Mistaken was compared to Fabricated, Evidence Strength did not affect the model. Adding Strong, $b=.77, S E=.50$, Wald $=2.40, p=.121$, Odds Ratio $=2.16$, or Weak evidence, $b=.37, S E=.49$, Wald $=.57, p=.450$, Odds Ratio $=1.44$, did not affect the likelihood that a participant would classify the alibi as Mistaken or as Fabricated, relative to when there was no physical evidence.

To follow up on whether location affected the alibi classifications that participants reported, the proportions of participants who indicated that the alibi was True, Mistaken, or Fabricated were compared between Iowa State and Ryerson. None of the comparisons were significant when the familywise error rate was adjusted using a Holm-Bonferroni procedure: True, $z=2.21, p=.027$; Fabricated, $z=-1.84, p=.066$; Mistaken, $z=-.78, p=.434$. However, there is a trend for more True classifications at Iowa State than at Ryerson.

Likelihood of suspect guilt. Participants also rated the likelihood that the suspect was guilty (see Table 11 for statistical results). Significant main effects were found for Evidence 
Strength and Location, with higher estimates at Ryerson than Iowa State, but these were qualified by a significant 3-way interaction. To probe this interaction, separate 3 (Evidence Strength: Strong, Weak, None) X 3 (Moral Desirability of Alibi Activities) ANOVAs were computed on the samples from Ryerson and from Iowa State (see Figure 3).

At Iowa State, Alibi Moral Desirability showed a significant main effect, $F(2,112)=$ $3.74, p=.027, \eta_{\mathrm{p}}^{2}=.063$, but Evidence Strength did not, $F(2,112)=0.21, p=.811, \eta_{\mathrm{p}}^{2}=.004$. The interaction between Alibi Moral Desirability and Evidence Strength approached significance, $F(4,112)=2.33, p=.060, \eta_{\mathrm{p}}^{2}=.077$. To examine this interaction, one-way ANOVAs were computed at each level of evidence strength, with moral desirability as the independent variable and likelihood of guilt as the dependent measure (see first panel of Figure 3).

Table 11

ANOVA results for participants' ratings of the likelihood of suspect guilt

\begin{tabular}{lcccc}
\hline Effect & $d f$ & $F$ & $p$ & $\eta_{\mathrm{p}}^{2}$ \\
\hline Alibi Content & 2,249 & 1.62 & .194 & .013 \\
Evidence Strength & 2,249 & 4.81 & .009 & .037 \\
Location & 1,249 & 10.58 & .001 & .041 \\
Alibi Content X Evidence Strength & 4,249 & 1.66 & .159 & .026 \\
Alibi Content X Location & 2,249 & 2.02 & .135 & .016 \\
Evidence Strength X Location & 2,249 & 2.48 & .086 & .020 \\
Alibi Content X Evidence Strength X Location & 4,249 & 3.41 & .010 & .052 \\
\hline
\end{tabular}

Alibi Moral Desirability did not exert a significant influence on judgments of likelihood of suspect guilt in the Strong Evidence, $F(2,42)=.20, p=.824, \eta_{\mathrm{p}}^{2}=.009$, or Weak Evidence conditions, $F(2,39)=.44, p=.646, \eta_{\mathrm{p}}^{2}=.024$, but it did in the No Evidence condition, $F(2,34)$ $=12.38, p<.001, \eta_{\mathrm{p}}^{2}=.421$. In the No Evidence condition, all pairwise comparisons were significant (see Figure 3): Morally Desirable vs. Neutral, $t(23)=4.58, p<.001, d=1.84$; 
Morally Undesirable vs. Morally Desirable, $t(23)=2.62, p=.015, d=1.27$; Neutral vs. Morally Undesirable, $t(22)=2.56, p=.018, d=1.05$. The likelihood of suspect guilt was evaluated as highest when the alibi described morally neutral activities and lowest when the alibi described morally desirable activities. The Morally Undesirable alibi was between these two and statistically significantly different from both. However, care is necessary in the interpretation of these findings due to the low cell sizes.

At Ryerson, opposite main effects were observed, with statistically significant differences observed for Evidence Strength, $F(2,137)=7.14, p=.001, \eta_{\mathrm{p}}^{2}=.094$, but not for Alibi Moral Desirability, $F(2,137)=.040, p=.961, \eta_{\mathrm{p}}^{2}=.001$. However, similar to the results from Iowa State, a significant 2-way interaction was observed, $F(4,137)=2.86, p=.026, \eta_{\mathrm{p}}^{2}=.077$ (see second panel of Figure 3). Again, Alibi Moral Desirability did not affect judgments when the physical evidence supporting the alibi was strong, $F(2,46)=.06, p=.945, \eta_{\mathrm{p}}^{2}=.002$, or weak, $F(2,44)=1.73, p=.189, \eta_{\mathrm{p}}^{2}=.073$, but the effect was significant when there was no physical evidence, $F(2,47)=3.32, p=.045, \eta_{\mathrm{p}}^{2}=.124$. In the No Evidence condition, only one comparison was statistically significant using the Holm-Bonferroni correction (see Figure 3); the probability of suspect guilt was rated as significantly higher in the Morally Undesirable condition than in the Neutral condition, $t(31)=2.62, p=.014, d=0.92$. Interestingly, this effect is in the opposite direction from the significant effect observed in the data from Iowa State, where the Neutral condition led to the highest likelihood of guilt ratings. The comparisons between the Morally Desirable and Neutral conditions, $t(32)=.33, p=.742, d=0.12$, and between Morally Undesirable and Morally Desirable conditions, $t(31)=1.97, p=.057, d=0.69$, did not achieve significance. As with the data from Iowa State, caution in interpretation is warranted due to the low cell sizes. 

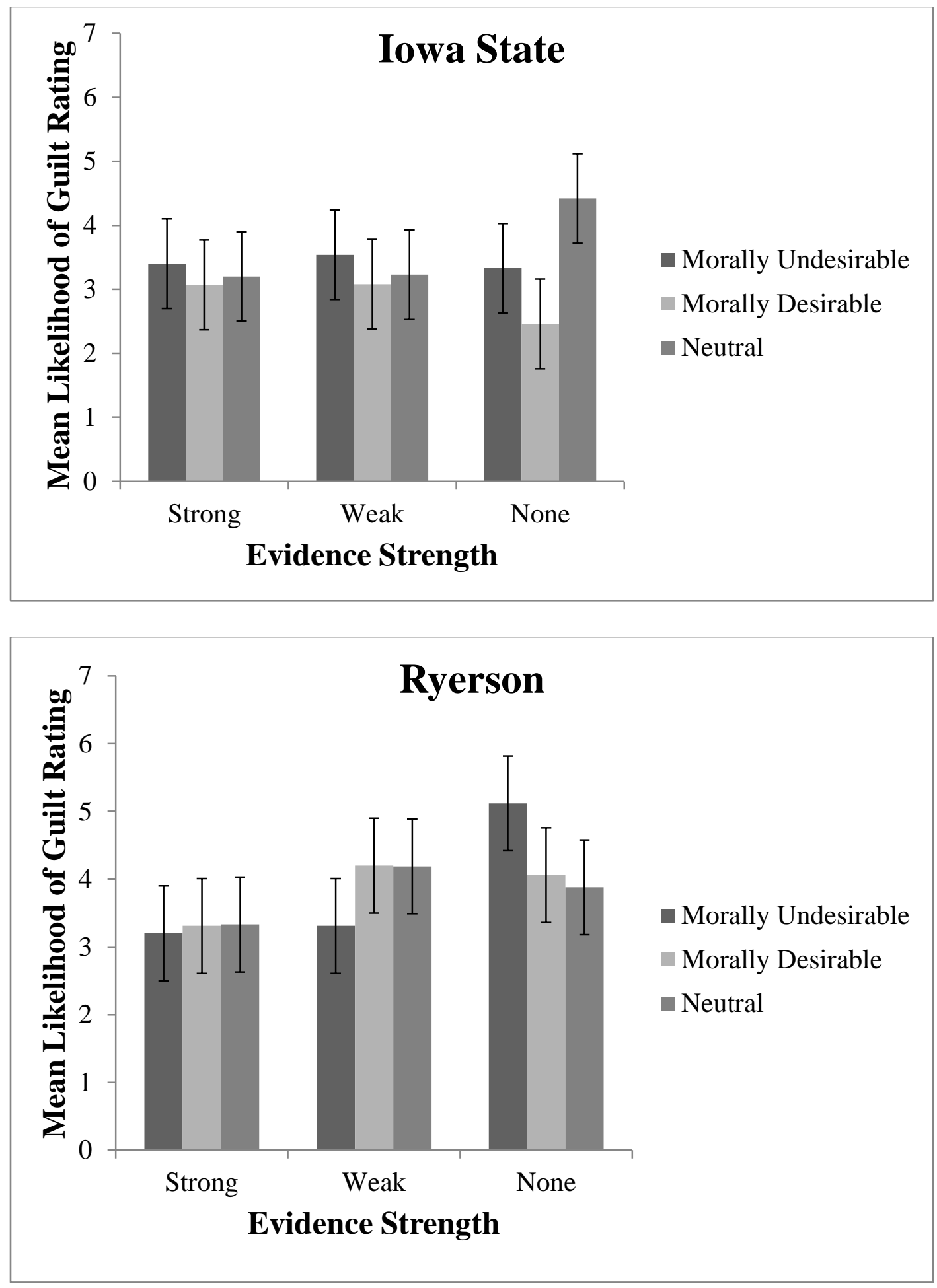

Figure 3. Mean ratings of the likelihood of suspect guilt for different alibi narratives and different levels of corroboration, at Iowa State and at Ryerson.

Verdict choice. Overall, $28.3 \%$ of participants provided guilty verdicts in the case. An 
ANOVA was also computed on participants' verdict choice to determine whether the independent variables affected verdict choice (see Table 12). This procedure is appropriate with dichotomous dependent variables when cell sizes are equal (Glass, Peckham, \& Sanders, 1972) and there is a minimum of 40 degrees of freedom in the error term (Lunney, 1970). Loglinear analysis is also appropriate for these data, but the results are often difficult to interpret when independent variables have more than two levels, as is the case here with Evidence Strength and Alibi Moral Desirability.

Table 12

ANOVA results for participants' verdict choice

\begin{tabular}{lcccc}
\hline Effect & $d f$ & $F$ & $p$ & $\eta_{\mathrm{p}}^{2}$ \\
\hline Alibi Content & 2,249 & .49 & .614 & .004 \\
Evidence Strength & 2,249 & 2.86 & .059 & .022 \\
Location & 1,249 & 4.71 & .031 & .019 \\
Alibi Content X Evidence Strength & 4,249 & .47 & .761 & .007 \\
Alibi Content X Location & 2,249 & 3.82 & .023 & .030 \\
Evidence Strength X Location & 2,249 & 3.97 & .02 & .031 \\
Alibi Content X Evidence Strength X Location & 4,249 & 1.27 & .284 & .020 \\
\hline
\end{tabular}

The only significant main effect was for Location, with more guilty verdicts at Ryerson than at Iowa State, though the main effect for Evidence Strength approached significance. These effects were qualified by two significant 2-way interactions: Alibi Moral Desirability X Location, and Evidence Strength X Location.

The significant interaction between Alibi Moral Desirability and Location was investigated by conducting separate univariate ANOVAs on the data from Iowa State and Ryerson, with Alibi Moral Desirability as the independent variable. The main effect of Alibi Moral Desirability was significant at Iowa State, $F(2,121)=3.30, p=.040, \eta_{\mathrm{p}}^{2}=.053$, and was 
not significant at Ryerson, $F(2,143)=.84, p=.424, \eta_{\mathrm{p}}^{2}=.012$. Descriptively ${ }^{12}$, at Iowa State, the highest proportion of guilty verdicts was found in the Neutral condition (13/40), and the lowest in the Morally Desirable condition (4/41), with the Morally Undesirable condition (8/40) between the two. This pattern replicates the pattern at Iowa State for likelihood of guilt ratings.

The significant interaction between Evidence Strength and Location was also examined by conducting separate ANOVAs on the data from Iowa State and Ryerson. The main effect of Evidence Strength was significant at Ryerson, $F(2,143)=7.01, p=.001, \eta_{\mathrm{p}}^{2}=.089$, but not at Iowa State, $F(2,118)=.07, p=.936, \eta_{\mathrm{p}}^{2}=.001$. At Ryerson, only the difference between Strong Evidence and No Evidence was significant, $z=3.55, p<.001$, with significantly fewer guilty verdicts in the Strong condition (8/49) than the No Evidence condition (25/50). No differences were observed between the Strong and Weak (14/47) conditions, $z=1.57, p=.117$, or between the Weak and No Evidence conditions, $z=2.03, p=.043$.

Participants' willingness to sign the petition. Overall, $52.8 \%$ of the participants included signed the petition. To examine whether rates of petition-signing were affected by Location, Alibi Moral Desirability, and Evidence Strength, an ANOVA was computed (see Table 13). The only significant effect was the interaction between all the independent variables (see Figure 4), though the interaction between Alibi Content and Location approached significance.

At Iowa State, the main effect for Alibi Moral Desirability was significant, $F(2,102)=$ $3.56, p=.032, \eta_{\mathrm{p}}^{2}=.065$, but the main effect for Evidence Strength, $F(2,102)=.06, p=.941, \eta_{\mathrm{p}}^{2}$ $=.001$, and the interaction between the two, $F(4,102)=1.62, p=.176, \eta_{\mathrm{p}}^{2}=.060$, were not significant. The main effect is driven by a significantly higher rate of petition-signing in the

\footnotetext{
${ }^{12}$ The calculation for the significance of the difference between two independent proportions requires that the $\mathrm{n}(\mathrm{p})$ and $n(1-p)$ - where $p$ refers to the proportion - both be greater than or equal to 5 . This requirement is not met for the proportion of guilty verdicts in the Morally Desirable condition
} 

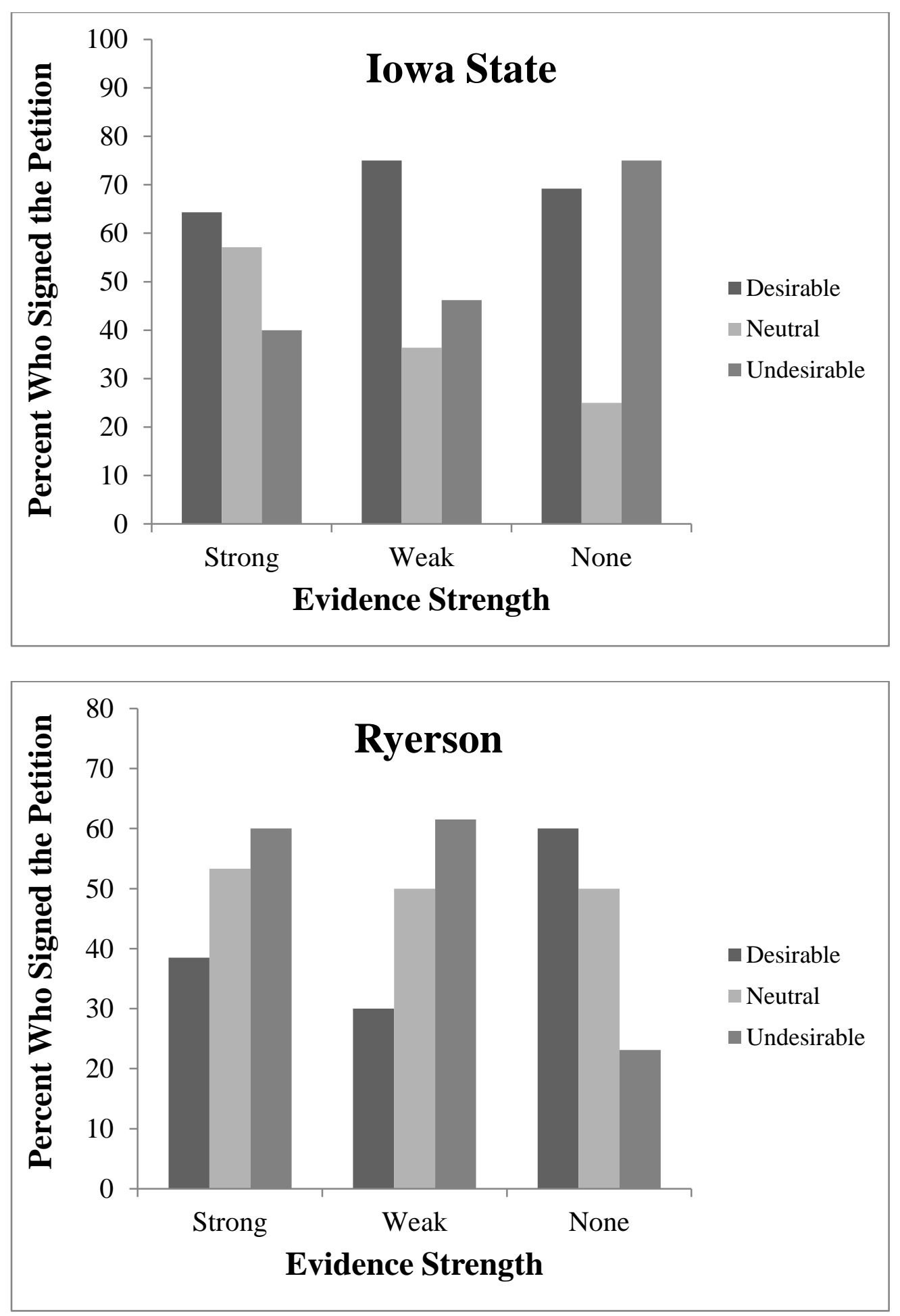

Figure 4. Percent of participants who signed the petition for different alibi narratives and different levels of corroboration, at Iowa State and at Ryerson. 
Table 13

ANOVA results for participants' willingness to sign the petition

\begin{tabular}{lcccc}
\hline Effect & $d f$ & $F$ & $p$ & $\eta_{\mathrm{p}}^{2}$ \\
\hline Alibi Content & 2,206 & .91 & .406 & .009 \\
Evidence Strength & 2,206 & .05 & .956 & $<.001$ \\
Location & 1,206 & 1.06 & .305 & .005 \\
Alibi Content X Evidence Strength & 4,206 & .75 & .561 & .014 \\
Alibi Content X Location & 2,206 & 2.82 & .062 & .027 \\
Evidence Strength X Location & 2,206 & .16 & .849 & .002 \\
Alibi Content X Evidence Strength X Location & 4,206 & 2.61 & .037 & .048 \\
\hline
\end{tabular}

Morally Desirable condition (27/39) than in the Neutral condition $(15 / 37), z=2.51, p=.012$.

The differences between the Morally Desirable and Morally Undesirable $(19 / 35), z=1.32, p=$ .186 , and the Morally Undesirable and Neutral conditions, $z=1.17, p=.243$, were not statistically significant. These results are consistent with results for likelihood of guilt ratings andverdict choice at Iowa State; providing an alibi that describes morally desirable activities is beneficial to the suspect, relative to morally neutral and morally undesirable alibis. In contrast, at Ryerson, there were no significant effects: Alibi Moral Desirability, $F(2,104)=.26, p=.769, \eta_{p}^{2}$ $=.005 ;$ Evidence Strength, $F(2,104)=.15, p=.863, \eta_{\mathrm{p}}^{2}=.003$; or the interaction between Alibi Moral Desirability and Evidence Strength, $F(4,104)=1.75, p=.144, \eta_{p}^{2}=.063$.

Explaining the location effects. An unanticipated finding in a previous section was the differences in results between the Iowa State and Ryerson samples, with participants from Iowa State providing generally more favourable responses than participants from Ryerson and also the different pattern of effects for Alibi Moral Desirability. This could be due to differences in the race/ethnicity and gender of the two groups (see Table 1). Additionally, it is possible that the differences in results between the two samples reflect an in-group bias. In general, individuals hold more favourable attitudes towards members of their in-group (i.e., a group to which they 
belong and feel an attachment, for example nationality) than individuals who are not in their ingroup (i.e., out-group members) (e.g., Taylor \& Doria, 1981). In this case, the materials implied that the suspect was American. This individual is more likely to be seen as an in-group member by participants at Iowa State than those at Ryerson. Although the study was not designed to test this hypothesis, exploratory analyses are possible using participants' ratings about how similar they believe they are to the suspect and how much they like the suspect.

The effect of participant racial/ethnic identity. The analyses in this section compared responses of White participants at Iowa State, White participants at Ryerson, and People of Colour (POC) at Ryerson. Previous literature (e.g., Cowan et al., 2015) has compared White and POC participants, and initial comparisons at Ryerson showed that this was statistically appropriate (see Appendix J). There were too few POC at Iowa State to include this group. Briefly, four significant effects were found (details and statistical results are in Appendix J): POC at Ryerson provided lower ratings of suspect honesty, higher ratings of the strength of the evidence against the suspect and likelihood of guilt, and more guilty verdicts than White participants at Iowa State. Ratings of White participants at Ryerson did not differ significantly from either group. No significant differences were found for either of the confidence reports, ratings of alibi accuracy, or petition-signing. Participant race/ethnicity may provide a partial explanation for the differences between the two samples.

The effect of participant gender. Analyses were computed with Participant Gender (male, female) and Location (Iowa State, Ryerson) as independent variables (see Appendix J for details and statistical results). Aside from some significant Location main effects, which were apparent in earlier analyses, the only effect of note was a marginal interaction for ratings of suspect honesty; ratings of female participants did not differ between Iowa State and Ryerson, whereas 
male participants at Iowa State provided higher ratings than male participants at Ryerson. Thus, participant gender does not explain the location effects.

In-group bias. It is also possible that the in-group bias based on nationality (i.e., American or Canadian) explains the differences in results between the Iowa State and Ryerson samples. During the study, participants rated how similar their personality is to the personality of the suspect and how much they like the suspect. Ratings of similarity were marginally significantly higher for participants from Iowa State $(M=2.33, S D=1.17)$ than for participants at Ryerson $(M=2.06, S D=1.30), t(298)=1.89, p=.060, d=0.22$. This result is consistent with in-group bias is operating. However, in general, participants rated themselves as being relatively dissimilar from the suspect. No significant differences were observed for ratings of how much the participants liked the suspect between Iowa State $(M=2.82, S D=1.13)$ and Ryerson $(M=$ 2.66, $S D=1.18), t(295)=1.12, p=.262, d=0.14$. Interestingly, participants' ratings of the similarity between themselves and the suspect and their ratings of how much they liked the suspect were correlated, $r=.532, p<.001$.

It is also possible that the effect for similarity ratings was due to the higher number of male participants at Iowa State. A 2 (Location: Iowa State, Ryerson) X 2 (Participant Gender: Male, Female) between-subjects ANOVA showed that male participants $(M=2.67, S D=1.33)$ rated themselves as more similar to the suspect than did female participants $(M=1.98, S D=$ $1.15), F(1,294)=18.13, p<.001, \eta_{\mathrm{p}}^{2}=.06$. The effect for Location was non-significant, $F(1$, $294)=.11, p=.740, \eta_{\mathrm{p}}^{2}<.001$, as was the interaction term, $F(1,294)=.63, p=.430, \eta_{\mathrm{p}}^{2}=.002$, suggesting that the effect noted above is due to gender differences between the samples rather than Location.

A series of regression models_-linear and binary logistic, as appropriate-were 
computed to investigate whether ratings of liking or similarity predicted participants' responses regarding legal aspects of the case. Initial regressions included an interaction term in a second step of the model, but this was consistently non-significant, never improved model fit, and did not influence the results, so the effect was omitted for the sake of parsimony. Detailed results are available in Tables 14 and 15. Participants' ratings of how similar they were to the suspect were unrelated to any of their judgments. However, greater liking of the suspect predicted: higher ratings of suspect honesty and statement accuracy; lower ratings of the strength of the evidence against the suspect and likelihood of guilt; fewer guilty verdicts; and more petition signing. Observed results do not clarify whether in-group bias may be the mechanism by which Location affected results. In all analyses, liking the suspect was associated with legal decisions that favoured the suspect (e.g., higher ratings of suspect honesty, lower probability of guilt ratings), but judgments of how similar the participant believed the suspect was to him/herself was not related to any of the outcomes. Thus, the results neither fully support, nor fully contradict the possibility that in-group bias was operating.

Evidence for implicit personality theories. One theory presented in the introduction suggested that participants form implicit personality theories based on positive or negative alibi information, and that these influence their legal judgments. Participants' perceptions of the similarity between themselves and the suspect, their liking for the suspect, and their overall evaluation of the suspect's character are indications of participants' overall assessment of the suspect. To explore this, 4 (Alibi Moral Desirability: Morally Desirable, Morally Undesirable, Neutral, No-Alibi Control) X 2 (Location: Iowa State, Ryerson) ANOVAs were computed on participants' ratings of similarity, liking, and their overall evaluations of the suspect. 
Table 14

Linear regression models using ratings of liking and similarity to predict legal judgments.

\begin{tabular}{|c|c|c|c|c|c|c|c|c|c|c|}
\hline \multirow[b]{2}{*}{ Outcome } & \multirow[b]{2}{*}{ Predictor } & \multicolumn{4}{|c|}{ Overall Model Fit } & \multicolumn{5}{|c|}{ Predictors } \\
\hline & & $d f$ & $F$ & $p$ & $R^{2}$ & $b$ & $S E(b)$ & $\beta$ & $t$ & $p$ \\
\hline \multirow[t]{3}{*}{ Suspect honesty } & & 2,294 & 14.25 & $<.001$ & .089 & & & & & \\
\hline & Liking & & & & & .29 & .08 & .23 & 3.54 & $<.001$ \\
\hline & Similarity & & & & & .12 & .08 & .10 & 1.50 & .135 \\
\hline \multirow{3}{*}{$\begin{array}{l}\text { Confidence in statement } \\
\text { honesty }\end{array}$} & & 2,294 & .71 & .494 & .005 & & & & & \\
\hline & Liking & & & & & .08 & .08 & .06 & .93 & .351 \\
\hline & Similarity & & & & & -.08 & .08 & -.08 & -1.12 & .263 \\
\hline \multirow[t]{3}{*}{ Accuracy } & & 2,260 & 7.88 & $<.001$ & .057 & & & & & \\
\hline & Liking & & & & & .18 & .09 & .14 & 1.91 & .057 \\
\hline & Similarity & & & & & .15 & .09 & .13 & 1.74 & .083 \\
\hline \multirow[t]{3}{*}{ Confidence in accuracy } & & 2,260 & .28 & .753 & .002 & & & & & \\
\hline & Liking & & & & & -.02 & .08 & -.02 & -.20 & .842 \\
\hline & Similarity & & & & & -.04 & .08 & -.04 & -.48 & .633 \\
\hline \multirow{3}{*}{$\begin{array}{c}\text { Strength of evidence } \\
\text { against suspect }\end{array}$} & & 2,293 & 6.71 & .001 & .044 & & & & & \\
\hline & Liking & & & & & -.23 & .09 & -.13 & -2.66 & .008 \\
\hline & Similarity & & & & & -.06 & .08 & -.05 & -.73 & .469 \\
\hline \multirow[t]{3}{*}{ Likelihood of Guilt } & & 2,294 & 7.84 & $<.001$ & .051 & & & & & \\
\hline & Liking & & & & & -.22 & .08 & -.18 & -2.64 & .009 \\
\hline & Similarity & & & & & -.09 & .08 & -.07 & -1.09 & .275 \\
\hline
\end{tabular}


Table 15

Binary logistic regression models using ratings of liking and similarity to predict verdict choice and petition signing

\begin{tabular}{|c|c|c|c|c|c|c|c|c|c|}
\hline \multirow[b]{2}{*}{ Outcome } & \multirow[b]{2}{*}{ Predictor } & \multicolumn{3}{|c|}{ Overall Model Fit } & \multicolumn{5}{|c|}{ Predictors } \\
\hline & & $\chi^{2}$ & $p$ & $R^{2}$ & $b$ & $S E(b)$ & Wald & $p$ & Odds Ratio \\
\hline \multirow[t]{3}{*}{ Verdict choice } & & 22.91 & $<.001$ & .106 & & & & & \\
\hline & Liking & & & & .46 & .14 & 10.70 & .001 & 1.58 \\
\hline & Similarity & & & & .15 & .136 & 1.25 & .264 & 1.16 \\
\hline \multirow[t]{3}{*}{ Petition signing } & & 6.43 & .040 & .03 & & & & & \\
\hline & Liking & & & & -.27 & .13 & 4.06 & .044 & .77 \\
\hline & Similarity & & & & -.04 & .12 & .09 & .764 & .97 \\
\hline
\end{tabular}

Note. Degrees of freedom for both analyses $=2$. Verdict choice was coded as $1=$ Guilty, $2=$ Not Guilty. Petition signing was coded as $1=$ Yes, 2 = No. 
Results revealed that Alibi Moral Desirability had a significant effect on participants ratings of how similar they believe they are to the suspect, $F(3,292)=8.90, p<.001, \eta_{\mathrm{p}}^{2}=.08$. The main effect for Location, $F(1,292)=3.45, p=.064, \eta_{\mathrm{p}}^{2}=.01$, and the interaction between Alibi Moral Desirability and Location, $F(3,292)=.33, p=.803, \eta_{\mathrm{p}}^{2}<.01$, did not reach significance. Descriptive statistics are shown in Table 16, and results of follow-up tests to probe the significant main effect for Alibi Moral Desirability are reported in Table 17. Participants rated themselves as significantly less similar to the suspect when he reported a Morally Undesirable alibi than any other alibi, or the Control. Similarity ratings did not vary between the other alibi conditions.

Alibi Moral Desirability also affected whether participants liked the suspect, $F(3,289)=$ $13.37, p<.001, \eta_{\mathrm{p}}^{2}=.12$. As in the preceding analysis, Location, $F(1,289)=2.58, p=.109, \eta_{\mathrm{p}}^{2}=$ .01 , and the interaction between the two, $F(3,289)=2.39, p<.069, \eta_{\mathrm{p}}^{2}=.02$, were not significant. Three pairwise comparisons for Alibi Moral Desirability were significant (see Table 17. Participants liked the suspect significantly less with the Morally Undesirable alibi than they did with the Neutral or Morally Desirable alibis. Although the pattern of means suggests that participants also liked the suspect less with the Morally Desirable alibi than they did in the Control condition, this comparison was not statistically significant. Additionally, participants liked the suspect significantly more when he provided the Morally Desirable alibi than the Neutral alibi. In general, the means followed the pattern that would be expected; they generally liked the suspect more when his alibi described Morally Desirable behaviour and liked him less when his alibi described Morally Undesirable behaviour. 
Table 16

Descriptive statistics for similarity, liking, and suspect's overall character by alibi condition

\begin{tabular}{lllll}
\hline Outcome & Alibi Condition & $M$ & $S D$ & $n$ \\
\hline Similarity & Morally Undesirable & 1.67 & 1.06 & 87 \\
& Neutral & 2.22 & 1.26 & 91 \\
& Morally Desirable & 2.52 & 1.23 & 89 \\
& Control & 2.58 & 1.30 & 33 \\
Liking & & & \\
& Morally Undesirable & 2.21 & 1.12 & 87 \\
& Neutral & 2.76 & 1.05 & 90 \\
& Morally Desirable & 3.23 & 1.09 & 88 \\
& Control & 2.75 & 1.16 & 32 \\
Is the suspect a bad & & & \\
or good person & & & \\
& Morally Undesirable & 4.64 & 1.64 & 87 \\
& Neutral & 5.52 & 1.21 & 91 \\
& Morally Desirable & 6.01 & 1.34 & 89 \\
& Control & 5.55 & 1.39 & 33 \\
\hline
\end{tabular}

Note. Similarity and liking were rated on a scale of 1-7. The overall evaluation of the suspect's character was rated on a scale from 1 (bad person) to 11 (good person).

Participants' ratings of the suspect's overall character were affected by Alibi Moral Desirability, $F(3,292)=2.58, p<.001, \eta_{\mathrm{p}}^{2}=.13$, but not by Location, $F(1,292)=.02, p=.904$, $\eta_{\mathrm{p}}^{2}<.01$, or by the interaction between Alibi Moral Desirability and Location, $F(3,292)=.61, p$ $=.610, \eta_{\mathrm{p}}^{2}=.01$. Four pairwise comparisons achieved significance (see Table 17). Participants in the Morally Undesirable condition rated the suspect's overall character as more negative than did participants in any of the other three conditions. Additionally, participants in the Neutral condition rated the suspect's overall character as more negative than participants in the Morally Desirable condition. Though, in general, participants had a neutral to somewhat negative view of the suspect (see Table 16). Taken together, these results are consistent with participants forming negative implicit personality theories about the suspect when the alibi was Morally Undesirable, but do not show strong evidence of positive implicit personality theories with the Morally 
Desirable alibi.

Table 17

Pairwise comparison of different levels of alibi moral desirability on similarity ratings

\begin{tabular}{|c|c|c|c|c|c|}
\hline Outcome & Comparison & $d f$ & $t$ & $p$ & $d$ \\
\hline \multicolumn{6}{|l|}{ Similarity } \\
\hline & Morally Undesirable - Neutral* & 173.27 & -3.17 & .002 & -0.48 \\
\hline & Morally Undesirable - Morally Desirable* & 171.61 & -4.92 & $<.001$ & -0.74 \\
\hline & Morally Undesirable - Control & 118 & -3.93 & $<.001$ & -0.80 \\
\hline & Neutral - Morally Desirable & 178 & -1.60 & .111 & -0.24 \\
\hline & Neutral - Control & 122 & -1.38 & .171 & -0.28 \\
\hline & Morally Desirable - Control & 120 & -.232 & .817 & -0.05 \\
\hline \multicolumn{6}{|l|}{ Liking } \\
\hline & Morally Undesirable - Neutral & 175 & -3.36 & .001 & -0.51 \\
\hline & Morally Undesirable - Morally Desirable & 173 & -6.10 & $<.001$ & -0.92 \\
\hline & Morally Undesirable - Control & 117 & -2.32 & .022 & -0.48 \\
\hline & Neutral - Morally Desirable & 176 & -2.94 & .004 & -0.46 \\
\hline & Neutral - Control & 120 & .03 & .890 & 0.01 \\
\hline & Morally Desirable - Control & 118 & 2.01 & .039 & 0.46 \\
\hline \multicolumn{6}{|c|}{$\begin{array}{l}\text { Is the suspect a } \\
\text { bad or good } \\
\text { person }\end{array}$} \\
\hline & Morally Undesirable - Neutral* & 157.84 & -4.04 & $<.001$ & -0.61 \\
\hline & Morally Undesirable - Morally Desirable* & 165.83 & -6.07 & $<.001$ & -0.92 \\
\hline & Morally Undesirable - Control* & 67.30 & -3.01 & .004 & -0.70 \\
\hline & Neutral - Morally Desirable & 178 & -2.61 & .010 & -0.39 \\
\hline & Neutral - Control & 122 & -.11 & .910 & -0.02 \\
\hline & Morally Desirable - Control & 120 & 1.69 & .093 & 0.34 \\
\hline
\end{tabular}

Note. Comparisons marked with a * did not meet the assumption of equality of variances, and the test that does not require this assumption is reported.

\section{Do perceptions of alibi moral desirability affect legal judgments through liking of the}

suspect? Although alibi moral desirability did not show a clear, direct effect on legal judgments in earlier analyses, it is possible that perceptions of alibi activities and the individuals who engage in such activities are related to legal judgments via ratings of liking the suspect.

Mediation analyses using Preacher and Hayes' (2008) method using bias-corrected bootstrapping with 10,000 replacements were conducted to determine whether mediation effects were present. The predictor was the average of participants' perceptions of the morality of the behaviour 
described in the alibi and an individual who performed this behaviour ${ }^{13}$. The outcomes were participants' legal judgments. And the mediator was participants' liking of the suspect (see Table 18 for statistical results). As participants in the Control condition did not provide ratings of the morality of the alibi behaviour and actor, they were not included in these analyses. Table 18

Results of mediation analyses

\begin{tabular}{|c|c|c|c|c|c|c|c|}
\hline \multirow[b]{2}{*}{ Outcome } & \multicolumn{2}{|c|}{$\begin{array}{c}\text { Total Effect } \\
(c)\end{array}$} & \multicolumn{2}{|c|}{$\begin{array}{c}\text { Direct Effect } \\
\left(c^{\prime}\right)\end{array}$} & \multicolumn{3}{|c|}{$\begin{array}{r}\underline{\text { Indirect Effect }} \\
95 \% \mathrm{CI}\end{array}$} \\
\hline & $\begin{array}{c}\text { Point } \\
\text { Estimate }\end{array}$ & $p$ & $\begin{array}{c}\text { Point } \\
\text { Estimate }\end{array}$ & $p$ & $\begin{array}{c}\text { Point } \\
\text { Estimate }\end{array}$ & CI LL & CI UL \\
\hline Statement honesty & .152 & $<.001$ & .100 & .015 & .052 & .017 & .095 \\
\hline $\begin{array}{l}\text { Confidence in statement } \\
\text { honesty }\end{array}$ & .028 & .465 & .017 & .692 & .011 & -.026 & .051 \\
\hline Accuracy & .149 & $<.001$ & .107 & .017 & .042 & .000 & .087 \\
\hline Confidence in accuracy & .073 & .044 & .098 & .015 & -.026 & -.063 & .009 \\
\hline $\begin{array}{c}\text { Strength of evidence } \\
\text { against suspect }\end{array}$ & -.117 & .004 & -.077 & .086 & -.040 & -.086 & .003 \\
\hline Likelihood of guilt & -.137 & .006 & -.087 & .047 & -.050 & -.097 & -.010 \\
\hline
\end{tabular}

Significant mediation effects were observed for participants' ratings of statement honesty and for ratings of likelihood of suspect guilt. The more the participants believed that the alibi behaviour was positive, the more they liked the suspect, $r=.447, p<.001$. Regression analyses presented earlier (see Table 14) established that greater liking of the suspect predicted higher estimates of statement honesty and lower estimates of likelihood of suspect guilt, even when ratings of similarity were controlled. However, mediation effects were not found for ratings of alibi accuracy, the strength of the evidence against the suspect, or for participants' confidence in

\footnotetext{
${ }^{13}$ This was used for three reasons. First, continuous variables are more powerful than categorical variables. Second, the alibi condition variable would need to be dummy coded, as it has three levels. This would require eliminating one comparison and generally leads to extra complication in interpreting the results. Finally, analyses on participants' perceptions of the moral desirability showed the pattern that would be expected, suggesting that the continuous variable is an appropriate substitute for the categorical variable in this instance
} 
their judgments. It seems that perceptions of alibi activities may show indirect effects on some legal judgments, not on others.

Authoritarianism. Authoritarianism is a second important area of inquiry for the current work. First, it was predicted that higher endorsement of Authoritarian ideals would be associated with judgments that are less favourable for the suspect (e.g., lower suspect honesty ratings, higher likelihood of guilt ratings). Second, it was anticipated that participants' impressions of the moral desirability of the alibi activities might mediate this relationship. The psychometric properties of the ACT were analyzed prior to inferential tests (see Appendix K). In total, 283 participants answered all questions on the ACT and were included in analyses. Three items performed poorly and were removed. Following this, alpha levels, inter-item, and item-total correlations were acceptable. In general, the scale performed well, a wide range of scores were observed, and mean scores were slightly below the midpoint.

Simple regressions. A series of simple regressions were computed to investigate whether participants' total and subscale scores on the ACT would predict their responses to the dependent variables. Statistical results are available in Table 19 and Table 20. Several patterns are worthy of note. First, participants' ratings of their confidence in their judgments about the honesty and the accuracy of the suspect's statements were not significantly predicted by any of their scores on the ACT. Second, in general, scores on the total ACT and its subscales were significantly negatively related to judgments of suspect honesty and suspect statement accuracy, and were significantly positively related to estimates of the strength of the evidence against the suspect and the likelihood of his guilt. Additionally, higher scores on the ACT were associated with a significantly higher likelihood of participants providing a guilty verdict and a significantly lower likelihood of choosing to sign the petition. As predicted, participants with higher scores were 
Table 19

Statistical results for linear regression models using ACT total and subscale scores to predict participants' responses on dependent measures

\begin{tabular}{|c|c|c|c|c|c|c|}
\hline Outcome & Predictor & $b$ & $S E(b)$ & $\beta$ & $R^{2}$ & $p$ \\
\hline \multicolumn{7}{|c|}{ Statement honesty } \\
\hline & $\mathrm{ACT}$ & -.016 & .006 & -.147 & .022 & .012 \\
\hline & Conservatism & -.025 & .016 & -.092 & .008 & .114 \\
\hline & Traditionalism & -.028 & .012 & -.132 & .017 & .024 \\
\hline & Authoritarianism & -.041 & .018 & -.132 & .018 & .022 \\
\hline \multicolumn{7}{|c|}{$\begin{array}{l}\text { Confidence in statement } \\
\text { honesty }\end{array}$} \\
\hline & $\mathrm{ACT}$ & .007 & .006 & .066 & .004 & .267 \\
\hline & Conservatism & .025 & .015 & .098 & .010 & .091 \\
\hline & Traditionalism & .003 & .012 & .015 & .000 & .795 \\
\hline & Authoritarianism & .017 & .017 & .045 & .003 & .335 \\
\hline \multicolumn{7}{|l|}{ Accuracy } \\
\hline & $\mathrm{ACT}$ & -.017 & .007 & -.162 & .026 & .010 \\
\hline & Conservatism & -.028 & .017 & -.106 & .011 & .088 \\
\hline & Traditionalism & -.035 & .013 & -.165 & .027 & .008 \\
\hline & Authoritarianism & -.035 & .019 & -.113 & .013 & .068 \\
\hline \multicolumn{7}{|c|}{ Confidence in accuracy } \\
\hline & $\mathrm{ACT}$ & .008 & .006 & .087 & .008 & .165 \\
\hline & Conservatism & .024 & .014 & .103 & .011 & .095 \\
\hline & Traditionalism & .010 & .012 & .052 & .003 & .409 \\
\hline & Authoritarianism & .010 & .017 & .035 & .001 & .574 \\
\hline \multicolumn{7}{|c|}{$\begin{array}{l}\text { Strength of evidence against } \\
\text { suspect }\end{array}$} \\
\hline & $\mathrm{ACT}$ & .031 & .006 & .291 & .085 & $<.001$ \\
\hline & Conservatism & .048 & .016 & .178 & .032 & .002 \\
\hline & Traditionalism & .048 & .012 & .221 & .049 & $<.001$ \\
\hline & Authoritarianism & .087 & .017 & .280 & .078 & $<.001$ \\
\hline \multicolumn{7}{|c|}{ Likelihood of Guilt } \\
\hline & $\mathrm{ACT}$ & .023 & .006 & .223 & .050 & $<.001$ \\
\hline & Conservatism & .041 & .015 & .156 & .024 & .007 \\
\hline & Traditionalism & .038 & .012 & .183 & .033 & .002 \\
\hline & Authoritarianism & .060 & .017 & .200 & .040 & .001 \\
\hline
\end{tabular}

more likely than those with lower scores to provide responses that favoured the prosecution.

Third, the Conservatism subscale was not a significant predictor of estimates of suspect honesty and the accuracy of the suspect's statement or decision to sign the petition, though it was 
significantly related to judgments of the strength of the evidence against the suspect and assessments of the likelihood of suspect guilt as well as verdict choice. And finally, the proportion of variance in the models that was accounted for by ACT total and subscale scores was notably higher for the strength of the evidence against the suspect and likelihood of suspect guilt than it was for the other linear regression models.

Table 20

Statistical results for binary logistic regression models using ACT total and subscale scores to predict participants' verdict choice and petition signing decision

\begin{tabular}{llcccccc}
\hline Outcome & Predictor & & & & & & $\begin{array}{l}\text { Odds } \\
\text { Ratio }\end{array}$ \\
\hline Verdict Choice & & $b$ & $S E(b)$ & Wald & $R^{2}$ & $p$ & \\
& ACT & -.048 & .011 & 18.93 & .104 & $<.001$ & .954 \\
& Conservatism & -.070 & .025 & 7.85 & .039 & .005 & .933 \\
& Traditionalism & -.067 & .020 & 11.95 & .060 & .001 & .935 \\
& Authoritarianism & -.143 & .032 & 20.26 & .109 & $<.001$ & .867 \\
& & & & & & & \\
Petition Signing & ACT & .020 & .010 & 4.47 & .025 & .035 & 1.020 \\
& Conservatism & .019 & .023 & .70 & .004 & .403 & 1.020 \\
& Traditionalism & .045 & .019 & 5.58 & .031 & .018 & 1.046 \\
& Authoritarianism & .055 & .028 & 3.91 & .021 & .048 & 1.056 \\
\hline
\end{tabular}

Note. The Nagelkerke $\mathrm{R}^{2}$ is reported. Also, note that verdict choice is coded as $1=$ Guilty, $2=$ Innocent. Petition Signing is coded as $1=$ Signed, $2=$ Not Signed.

Mediation effects of alibi moral desirability. One of the research questions for this project was whether participants' evaluation of the moral desirability of alibi activities mediates the relationship between Authoritarianism and participants' legal judgments (e.g., statement honesty, likelihood of guilt). It was predicted that individuals who scored higher on the ACT would be more likely to view the Morally Undesirable alibi negatively and reject the statement, whereas individuals who scored lower on the ACT would be more likely to interpret a Morally Undesirable alibi as indicating honesty and accept the statement. To investigate this, a series of mediation analyses were conducted using Preacher and Hayes' (2008) bias-corrected 
bootstrapping method with 10,000 replacements. In all analyses, ACT scores were the predictor, participants' legal judgments were the outcome, and participants' evaluations of morality were the mediator. The mediator was the average of participants' ratings for the suspect's alibi behaviour and an adult male who had engaged in this behaviour ${ }^{14}$. Lower scores indicate negative views. Control participants did not provide these ratings and are excluded from these analyses. Statistical results are presented in Table 21. Unfortunately, these analyses cannot be easily conducted on categorical outcomes, so these were not computed.

All of the total effects (c) except one are consistent with the results of the simple regression analyses. Significant mediation effects - indicated when the confidence interval of the indirect effect does not include zero - were observed for five out of six analyses that included the Authoritarianism subscale. Simple relationships (i.e., total $[c]$ and direct $\left[c^{\prime}\right]$ effects were significant) were observed in two of the five models where mediation was present.

In the first step of all the mediation effects, higher scores on the Authoritarianism subscale were associated with less favourable responses to the alibi behaviour and the actor carrying it out, $r=-.139, p<.001$. Participants' responses to the alibi behaviour and the actor were positively correlated with estimates of statement honesty, $r=.247, p<.001$, alibi accuracy, $r=.225, p<.001$, and confidence in alibi accuracy judgments, $r=.127, p=.040$. Participants' responses to the alibi behaviour and actor are negatively correlated with judgments of the strength of the evidence against the suspect, $r=-.174, p=.005$, and likelihood of suspect guilt, $r$ $=-.211, p=.001$. Overall, higher Authoritarianism scores were associated with more negative reactions to the alibi behaviour, which led to responses that are less favourable for the suspect.

\footnotetext{
${ }^{14}$ As the results of the manipulation checks showed significant differences in these two responses between the Alibi Moral Desirability, they are appropriate as a proxy for experimental condition, and are preferable for these analyses because they are continuous variables.
} 
Table 21

Results of mediation analyses

\begin{tabular}{|c|c|c|c|c|c|c|c|c|}
\hline \multirow{4}{*}{ Outcome } & \multirow{4}{*}{ Predictor } & \multirow{2}{*}{\multicolumn{2}{|c|}{$\begin{array}{l}\text { Total Effect } \\
(c)\end{array}$}} & \multicolumn{2}{|c|}{$\begin{array}{c}\text { Direct Effect } \\
\left(c^{\prime}\right)\end{array}$} & \multicolumn{3}{|c|}{$\underline{\text { Indirect Effect }}$} \\
\hline & & & & & & \multicolumn{3}{|c|}{$95 \%$ CI } \\
\hline & & Point & & Point & & Point & CI LL & CI UL \\
\hline & & Estimate & $p$ & Estimate & $p$ & Estimate & & \\
\hline \multicolumn{9}{|c|}{ Statement honesty } \\
\hline & ACT & -.014 & .021 & -.012 & .049 & -.002 & -.005 & .000 \\
\hline & Conservatism & -.021 & .177 & -.020 & .187 & -.001 & -.010 & .006 \\
\hline & Traditionalism & -.030 & .014 & -.026 & .029 & -.004 & -.012 & .001 \\
\hline & Authoritarianism & -.030 & .090 & -.021 & .234 & -.009 & -.022 & -.002 \\
\hline \multicolumn{9}{|c|}{$\begin{array}{l}\text { Confidence in statement } \\
\text { honesty }\end{array}$} \\
\hline & $\mathrm{ACT}$ & .004 & .516 & .005 & .454 & -.001 & -.004 & .001 \\
\hline & Conservatism & .016 & .317 & .016 & .310 & .000 & -.004 & .001 \\
\hline & Traditionalism & .002 & .900 & .003 & .835 & -.001 & -.007 & .001 \\
\hline & Authoritarianism & -012 & .662 & .014 & .795 & -.003 & -.012 & .002 \\
\hline \multicolumn{9}{|l|}{ Accuracy } \\
\hline & $\mathrm{ACT}$ & -.018 & .009 & -.015 & .023 & -.002 & -.007 & .001 \\
\hline & Conservatism & -.030 & .075 & -.029 & .077 & -.001 & -.010 & .004 \\
\hline & Traditionalism & -.035 & .008 & -.031 & .016 & -.004 & -.013 & .001 \\
\hline & Authoritarianism & -.036 & .065 & -.026 & .170 & -.010 & -.023 & -.002 \\
\hline \multicolumn{9}{|c|}{ Confidence in accuracy } \\
\hline & $\mathrm{ACT}$ & .008 & .151 & .010 & .091 & -.002 & -.005 & .000 \\
\hline & Conservatism & .024 & .095 & .025 & .086 & -.001 & -.006 & .004 \\
\hline & Traditionalism & .010 & .402 & .012 & .300 & -.002 & -.009 & .001 \\
\hline & Authoritarianism & .010 & .538 & .016 & .341 & -.006 & -.015 & -.001 \\
\hline \multicolumn{9}{|c|}{$\begin{array}{c}\text { Strength of evidence } \\
\text { against suspect }\end{array}$} \\
\hline & $\mathrm{ACT}$ & .034 & $<.001$ & .032 & $<.001$ & .002 & .000 & .004 \\
\hline
\end{tabular}




\begin{tabular}{llllllllll}
\hline & Conservatism & .055 & .001 & .054 & .001 & .001 & -.005 & .008 \\
& Traditionalism & .048 & $<.001$ & .046 & $<.001$ & .003 & -.001 & .010 \\
& Authoritarianism & .095 & $<.001$ & .090 & $<.001$ & .006 & .001 & .017 \\
Likelihood of guilt & & & & & & & & \\
& & & & & & & & & \\
& ACT & .025 & $<.001$ & .022 & .001 & .002 & .000 & .006 \\
& Conservatism & .043 & .008 & .042 & .008 & .001 & -.006 & .009 \\
& Traditionalism & .038 & .004 & .034 & .007 & .004 & -.001 & .011 \\
& Authoritarianism & .070 & $<.001$ & .062 & .001 & .008 & .002 & .019 \\
\hline
\end{tabular}


Qualitative analysis of participants' definitions of an "alibi." A final aim of this work was to replicate Cowan's (2012) finding that many participants misunderstood the term "alibi." The legal definitions of the term, and the definition used in social science research, generally emphasizes that an alibi is a narrative. Thus, when a suspect reports being somewhere else when a crime occurred, this constitutes his or her alibi, even when there is no corroboration available. However, Cowan (2012) found that participants often incorrectly reported that an alibi was the corroborative evidence, rather than the narrative itself, for example noting that the other patrons at the bar would be the suspect's alibi. In fact, the statement about being at the bar would be the alibi and the other patrons would be more accurately described as potential alibi corroborators or witnesses. Consequently, participants provided a definition for the term "alibi" for qualitative analysis using a template coding approach (see Appendix L). The two coders achieved acceptable inter-rater reliability, assigning identical codes for $85.7 \%$ of participant's responses, and showing partial agreement for $2.7 \%$ of participants' responses. Code numbers referenced in the text refer to the codes in the codebook.

Over a third $(39.3 \%)$ of participants indicated that an alibi refers to evidence supporting a narrative, rather than to the narrative itself (code 5 in codebook). These participants generally referenced alibi witnesses, though some also mentioned physical evidence. Some of these participants also indicated that an alibi would need to be investigated or that its veracity is uncertain (code 6).

Interestingly, $10.6 \%$ of responses suggested that participants either were entirely unaware of the definition, or, in a small number of cases, were highly uncertain (code 9). In the majority of cases, participants' responses were either so unclear that it was impossible to ascertain how they understood the term "alibi." In others, the participants clearly misunderstood the term, for 
example "Person who decides to do nothing when a negative action or speech is encountered" or "a group of people who have your back at all times." The high percentage of participants who focused on evidence, above, combined with the number of participants whose responses suggested clear misunderstandings - approximately half of the responses - suggests that a high number of participants do not understand the term "alibi."

Approximately a quarter (23.3\%) of the responses made primary reference to an alibi being a statement about one's whereabouts during the time of a crime (code 1). This would be considered an accurate response. In addition, $13.0 \%$ of responses indicated that an alibi was a narrative, but did not make specific reference to a crime (code 2). Approximately one in ten participants indicated that an alibi could be true or false and/or should/would be investigated (11.0\%; code 6) and that an alibi should be supported by evidence (11.3\%; code 11$)$. The remaining codes appeared in 5\% or fewer of participants' statements (code 4: $0 \%$; code 7: 1.7\%; code 8: $4 \%$; and code 10: $3.7 \%$ ).

Inferential tests were computed to determine whether participants' different understandings about the term "alibi" (i.e., a narrative vs evidence) affected their judgments about the legal aspects of the case. No significant differences were observed (see Appendix M).

\section{Discussion}

Experiment 1 provided insight on a number of issues. First, suspects who had or did not have alibis were compared. Second, the influence of alibi moral desirability and the strength of the evidence supporting an alibi was explored for legal judgments. Third, the effect of alibi moral desirability on non-legal judgments, like liking the suspect, was examined. Theoretical implications of these results are discussed. Fourth, Authoritarianism was investigated. Finally, participants" definitions of the term "alibi" were explored. 


\section{Having an alibi is preferable to not having an alibi. Initial analyses comparing}

responses of participants whose materials included an alibi to those whose materials did not include an alibi (i.e., No-Alibi Control) showed that having an alibi was helpful for suspects for nearly all outcomes that were measured. The exceptions were for rates of guilty verdicts and participants' willingness to sign a petition in support of the suspect. This could be due to inadequate statistical power to find results for dichotomous outcomes or due to participant uncertainty. Likelihood of guilt ratings were generally near the midpoint of the scale, even when an alibi was present, suggesting that participants were relatively uncertain about the suspect's guilt or innocence. This is consistent with Olson and Wells' (2004) observation that even the strongest alibi that they provided to participants was still viewed with some suspicion. Participants' threshold of guilt beyond a reasonable doubt that is required in order for them to vote guilty may not have been met. Similarly, this uncertainty about the suspect's guilt, even among those who read an alibi, may have prevented the presence of an alibi from affecting petition-signing behaviour. Thus, it appears that having an alibi is helpful, but it may not be sufficient to truly remove a suspect from suspicion, or to influence individuals' behaviour with respect to the suspect.

When the specific alibi narratives were compared to the control condition, weak support was found to suggest that a Morally Desirable alibi might be preferable to the other alibis. Significant effects were found for some, but not all, dependent measures. In general, when an overall effect for Alibi Moral Desirability was found, at least one of the alibis led to ratings that were more favourable for the suspect than when there was no alibi present. Interestingly, when some, but not all, comparisons were significant, the overall effect appears to be driven by the Morally Desirable alibi. 


\section{The effect of moral desirability on legal judgments and its interaction with evidence}

strength. Alibi Moral Desirability showed a mix of non-significant (statement honesty, alibi accuracy, confidence ratings, and alibi classification) and complex significant effects (strength of evidence against the suspect, likelihood of guilt, verdict choice, petition-signing). Interestingly, Alibi Moral Desirability was more likely to affect judgments from participants in the Iowa State sample than from participants from Ryerson, and when there was no physical evidence to support the alibi. The pattern of effects differed by site. As one example, at Iowa State, participants thought it was most likely that the suspect was guilty when his alibi was Neutral and least likely when his alibi was Morally Desirable. The Morally Undesirable alibi was between these two. In contrast, at Ryerson, participants indicated that it was less likely that the suspect was guilty when his alibi was Neutral than when it was Morally Undesirable. The alibi that is least favourable to the suspect at Iowa State (i.e., Neutral) is the most favourable alibi at Ryerson. At Iowa State, the Morally Undesirable alibi was more favourable for the suspect than the Neutral alibi, but less favourable than the Morally Neutral one, whereas at Ryerson, the Morally Undesirable alibi was the least favourable for the suspect. At present, an appropriate tentative conclusion is that Alibi Moral Desirability may influence judgments, but that this generally occurs only in particular contexts, most notably when there is no physical evidence to substantiate an alibi statement.

As well, some support was found to suggest that participants' own perceptions of the alibi behaviour might influence some of their legal judgments indirectly. Participants reported that they were less similar to the suspect and they rated his overall character lower when he presented a Morally Undesirable alibi compared to any of the other conditions. They also liked the suspect least when his alibi was Morally Undesirable and most when his alibi was Morally 
Desirable. Mediation analyses also showed that the more positively participants rated the behaviour described in the alibi and an individual who might engage in the behaviour, the more they liked the suspect. The more they liked the suspect, the more honest they thought his statement was and the less likely they thought it was that he was guilty. Taken together, these results all suggest that alibi moral desirability may affect legal judgments, but that the influence is affected by contextual factors (e.g., Location), and other judgments (e.g., Liking).

It is also worth noting that the results of Experiment 1 generally replicated the results from Olson and Wells (2004) with respect to alibi corroboration by physical evidence. Evidence strength affected ratings of suspect honesty, alibi accuracy, verdict choice, alibi classification, and likelihood of suspect guilt. Strong — and sometimes Weak — evidence was beneficial for suspects, relative to having no evidence. In particular, the effect of Alibi Moral Desirability was only found for likelihood of guilt judgments when there was No Evidence to corroborate the alibi. Finally, in terms of alibi classification, presenting physical evidence to support an alibi statement may lead evaluators to shift their judgments from Fabricated to True, but not from Fabricated to Mistaken. This is logical, as it would be odd to find compelling physical evidence to support a mistaken alibi, where a suspect is attempting to provide an honest account of his/her whereabouts, but a memory error occurs. For example, if an innocent suspect reports being at work at the time of a crime, but was actually at a baseball game, it would be highly unusual to find a timecard showing that s/he was at work. In general, it seems as though Alibi Evidence Strength has a stronger, more consistent effect on participants' judgments than does Alibi Moral Desirability. 


\section{The effect of alibi narratives on ratings of similarity, liking, and the overall}

evaluation of the suspect's character, and mediational effects of the former. Although the majority of analyses suggested that alibi moral desirability has, at best, complex effects on legal judgments, results on participants' ratings of similarity between themselves and the suspect and liking the suspect show that alibi moral desirability may affect other types of judgments. Indeed, participants rated themselves as less similar to the suspect when he provided a morally undesirable alibi compared to the other alibis, or the control condition. Additionally, they liked the suspect less when he provided an alibi that described morally undesirable behaviour than with the other two alibis. Participants liked the suspect more when the alibi was morally desirable than when it was neutral. All of these effects were independent of sample location.

In general, the more participants liked the suspect, the more favourable their legal judgments ratings were for him. Similarity ratings were not related to legal judgments. Given that alibi moral desirability affected liking, and liking was associated with legal judgments, it was proposed that participants' perceptions of alibi moral desirability might show an indirect effect on legal judgments through liking the suspect. As noted earlier, two mediation effects of six were significant. In general, it seems as though the moral desirability of alibi activities may affect some legal judgments indirectly by affecting how much evaluators like the suspect, but the effects are not consistent.

\section{Differences between the Ryerson University and Iowa State University samples.} Analyses revealed a pattern of differences in results between the two locations where data were collected. In particular, participants at Iowa State generally provided responses that were more favourable to the suspect, and were more likely than those at Ryerson to be influenced by Alibi Moral Desirability. In general, participants at Iowa State responded most favourably to the 
suspect when his alibi described Morally Desirable activities, and least favourably when his alibi was morally Neutral. In contrast, in the few circumstances when participants at Ryerson were influenced by Alibi Moral Desirability, their responses were more favourable towards the suspect when his alibi was Neutral than when it was Morally Undesirable. These differences were not hypothesized and were entirely unanticipated. As a result, it is only possible to offer post-hoc theorizing based on available data and well-established effects from the literature. Three possibilities were considered to explain the location effects: differences in the racial/ethnic composition of the two samples; differences in the gender makeup of the two samples; and ingroup bias. It is also possible that other differences that could not be explored with the study's design were responsible for the results, for example population (Toronto: 2.6 million; Greater Toronto Area: 6.1 million; Ames: 62,000) or level of religiosity.

Some support was found for the notion that racial/ethnic differences were responsible for the different patterns of results observed at Iowa State and at Ryerson. The sample at Iowa State was almost exclusively White, whereas only approximately a third of participants at Ryerson were White. Where significant differences were observed, White participants at Iowa State provided responses that were more beneficial to the suspect than ratings of POC (People of Colour) at Ryerson. White participants at Ryerson provided ratings that were not significantly different from either group. However, this effect was not found for all outcomes; interestingly, significant effects for participant race/ethnicity were found for outcomes that showed main effects for Location (e.g., suspect honesty, likelihood of guilt), but not for outcomes where there was no significant main effect of Location (e.g., confidence ratings, alibi accuracy ratings).

It is also possible that participant gender affected results and is responsible for the differences in results observed between sample locations. The sample at Ryerson included 
mostly women $(81.5 \%)$, whereas the sample at Iowa State had a relatively even gender split (56.5\% women, $43.5 \%$ men). This hypothesis was not supported in follow-up analyses; participant gender was only implicated in one marginally significant interaction.

Finally, in-group bias suggests that individuals hold more positive attitudes towards people who are also members of groups with which the individual feels an affiliation (i.e., ingroup members) than towards those who are not members of these groups (i.e., out-group members (e.g., Taylor \& Doria, 1981). The experimental materials for all participants described a case that was set in Seattle, WA, and, presumably, involved an American suspect. This suspect, then, is more likely to be seen as an in-group member by American participants at Iowa State than by Canadian participants at Ryerson. Although Experiment 1 was not designed to consider this possibility specifically, it is possible to gain some insight based on participants' ratings of the perceived similarity between themselves and the suspect and their ratings of how much they like the suspect. In general, individuals will see members of their in-groups as more similar to themselves than members of their out-groups (e.g., Wilder, 1984). In fact, members of in-groups do tend to be more similar to each other than they are to out-group members (e.g., George, 1990; Levine \& Moreland, 1998; Magaro \& Ashbrook, 1985). As well, individuals also like in-group members more than out-group members (e.g., Turner, Brown, \& Tajfel, 1979). Perceived similarity tends to lead to liking (e.g., Sprecher, 2014), even among children as young as three years (e.g., Fawcett \& Markson, 2010). Interestingly, liking appears to also lead to higher ratings of similarity (e.g., Collisson \& Howell, 2014). Thus, participants' ratings of how similar they believe they are to the suspect and how much they like the suspect can provide some tentative indications regarding whether in-group bias may be the mechanism by which sample location affected the results. 
Observed results show that ratings of similarity between the participant and the suspect were significantly higher at Iowa State than at Ryerson, though this seems to be due to the greater number of male participants at Iowa State than at Ryerson. However, participants' ratings of similarity were not predictive of their judgments about the case in regression models. It is possible that these non-significant findings are due to restriction of range, rather than a genuine absence of an effect; all participants rated themselves as fairly dissimilar from the suspect. Participants were only incidentally exposed to information about the suspect through the case materials, as the study's focus was on the effect of aspects of the alibi rather than aspects of the suspect. The only verifiable information they were likely to glean was that the suspect was a male who was presumably American, and presumably was an adult, though they may have made inferences about his personality or character based on his language in the transcript and his alibi activities. These inferences should not have varied systematically between sample locations. Regardless, participants had little information about his personality or general behaviour with which to make this judgment.

No significant difference was observed between ratings of liking the suspect between the Iowa State and Ryerson samples. However, participants' liking of the suspect did affect their legal judgments, with the exception of the two confidence ratings. The effect was remarkably consistent across the different outcome measures. The more participants liked the suspect, the more favourable their legal judgments were (e.g., higher suspect honesty, lower likelihood of guilt). Taken together, the current results neither confirm nor disconfirm whether in-group bias was operating and provide an explanation for the location effects. Further research is needed to investigate whether in-group bias may affect judgments about alibis specifically or legal judgments in general. 


\section{Authoritarianism and the mediational effects of perceived alibi moral desirability.}

In general, total scores on the ACT (Duckitt et al., 2010) and its subscales - with one problematic item removed from each subscale - significantly predicted all of the outcome variables except for participants' self-reported confidence in their judgments about the honesty of the suspect's statement and the accuracy of his alibi. In all cases, the higher the scores participants received on the ACT, the less likely it was for their ratings and decisions to be beneficial for the suspect. This is entirely consistent with Cowan's (2012) results, using a revised version of Altemeyer's (1981) RWA Scale. It is also consistent with existing literature showing that Authoritarianism is positively correlated with a greater number of guilty verdicts and convictions (Devine, Clayton, Dunford, Seying, \& Price, 2001; Narby, Cutler, \& Moran, 1993), longer recommended sentences (Devine et al., 2001), support for capital punishment (McKee \& Feather, 2008) and more skepticism of alibis (Cowan, 2012; Culhane, 2005). In the literature greater endorsement of Authoritarian beliefs are related to outcomes that are consistently less favourable for a suspect or defendant. The results also provide evidence of convergent validity for the ACT, as the same pattern of results was observed for the ACT and the RWA in the current results and Cowan's (2012) results, using very similar experimental materials.

It was also expected that higher endorsement of Authoritarian beliefs would lead evaluators to be more likely to judge a suspect's negative alibi behaviour harshly than evaluators who are low on Authoritarianism and, subsequently, to treat the alibi provider more harshly. A morally undesirable alibi is a violation of traditional social values, and individuals high on Authoritarianism - as a general construct - are generally more likely to adhere to traditional social norms, obey legitimate authorities, and wish to reject and punish those who violate these social values. Results of mediation analyses support this assertion, but only for the 
Authoritarianism subscale of the ACT. Higher scores on this subscale indicate support for harsh, punitive measures to achieve social control and protect against threats to safety and security. Higher scores on the Authoritarianism subscale were significantly associated with more negative views about the alibi behaviour and an individual who had engaged in that behaviour, as would be expected. Negative views about the behaviour and actor were associated with lower estimates of statement honesty and accuracy, less confidence in alibi accuracy judgments, and higher estimates of the strength of the evidence against the suspect and the likelihood of suspect guilt. These results are consistent with the hypothesis that the tendency for individuals who are high in Authoritarianism - at least as it relates to a desire to reject and punish transgressors - to provide legal judgments that are less favourable to suspects is attributable, at least in part, to a tendency to rate their alibi behaviours more negatively than individuals who are low in Authoritarianism. It seems that individuals who are high in Authoritarianism may be more likely than those who are low in Authoritarianism to form an implicit personality theory about a suspect and judge him or her consistently with that theory.

Theoretical considerations. Previous theoretical and empirical work suggested two possible patterns of results for how the moral desirability of activities described in an alibi might affect participants' judgments. One theory, supported by well-established social psychology principles, suggests that a morally undesirable alibi will lead evaluators to form a negative implicit personality theory about the alibi provider and, consequently believe his or her alibi statement less than a neutral alibi. The same process would operate with an alibi describing morally desirable activities, though the implicit personality theory would be positive, and the alibi would be more likely to be believed. In this case, results should show that the Morally Desirable alibi was the most advantageous for the suspect and the Morally Undesirable one was 
the least advantageous. The second theory, which has some support from research on the influence of alibi salaciousness on legal judgments, suggests that when a suspect reveals negative behaviour in his or her alibi, this will be interpreted as a sign of honesty, and the alibi will be believed more. The effect of providing a morally desirable alibi under this theory is not clear.

The results from Iowa State were at least partly consistent with both theories. The Morally Desirable alibi led to significantly lower likelihood of guilt ratings than for the Neutral alibi and the Morally Undesirable alibi. This was consistent with the first theory, as reporting positive behaviour in an alibi was more advantageous for the suspect than neutral activities. Yet, the Morally Undesirable alibi was also more beneficial to the suspect than the Neutral alibi. This was not consistent with the prediction that participants would form a negative implicit personality theory about an alibi provider that revealed bad behaviour in an alibi and disbelieve his or her statement. It was, however, consistent with the second theory, which suggested that revealing bad behaviour would be taken as a sign of honesty. This same pattern was observed for Iowa State participants' verdict choices and their decision to sign the petition. In contrast, the data from Ryerson on likelihood of guilt judgments suggested that participants formed a negative implicit personality theory when the suspect described morally undesirable behaviour in his alibi. There was no evidence of a positive implicit personality theory for morally desirable alibis. Additionally, as discussed above, the mediation analyses - which were not computed separately based on location due to concerns about statistical power - provided some support for the suggestion that whether participants form an implicit personality theory or treat an admission of bad behaviour as an indication of honesty may depend on the evaluator's endorsement of Authoritarian beliefs about punishment. Overall, the data provide some support for both theories, and results suggest that different processes may operate in different social contexts and 
dependent on the evaluator's personality.

Participants' understanding of the term “alibi." Previous research by Cowan (2012) showed high variability and many misunderstandings in how participants understood the term "alibi." The current results supported these findings. In the legal context, the term "alibi" is used to reference a claim that an individual was elsewhere when a crime was committed (Burke et al., 2007), with the implication being that it would be physically impossible for the individual to have committed the crime. The definition provided in $R v$ Gottschall also discusses only a narrative. In contrast, nearly $40 \%$ of participants provided definitions that focused primarily exclusively on the evidence. In particular, these participants indicated that one's alibi was a witness, which is consistent with colloquial use of the term (e.g., "s/he is my alibi"). In contrast, only approximately a quarter of participants' responses considered an "alibi" to be the statement that a suspect offered describing his/her whereabouts at the time of the crime, which is consistent with Gottschall and the definition of the term used in social science. Participants who focused on narratives did also sometimes discuss the need for an alibi to be investigated or its veracity established, or that it is beneficial to have evidence to support an alibi narrative. Interestingly, although inconsistency in participants' definitions was observed, their legal judgments were unaffected by whether participants thought of an alibi as a narrative regarding a suspect's whereabouts at the time of a crime or as evidence supporting a narrative.

Limitations. One significant limitation was the design of the petition website. A number of participants indicated in debriefing that the petition was not realistic. In particular, some participants noted that the website URL was not what would be expected from a site that hosts many petitions. The URL provided was www.importantpetitions.com, implying that the petition was hosted on the organization's homepage. Rather, participants expected to see a URL that 
included a string of characters after the primary part of the URL (e.g., www.importantpetitions.com/nsdow49-sdkj38-bhsoidnmv). They also noted that the design of the website appeared rather sparse and lacked some of the features they had come to expect from petition websites, like banner advertisements, links to share the petition on social media sites, et cetera. And finally, a few participants discovered that the link to the website's security and privacy policy was not functional, which aroused their suspicion. Although few participants $(6.0 \%)$ reported being suspicious enough to justify removing their data from analysis, it is possible that the remaining participants were suspicious enough that their behaviour was affected.

A second important limitation is that the experiment was not designed to test and explain differences in the results based on sample location. These observed differences were not hypothesized and were unexpected. As a result, the explanations that have been offered are based on post-hoc theorizing stemming from the observed results and established social psychology findings rather than testing an a priori hypothesis. Some evidence was found to support the contention that participant race/ethnicity affected the results, though finding only differences between People of Colour (POC) at Ryerson and White participants at Iowa State suggests that the differences are not attributable only to differences in race/ethnicity across the two samples. In a pure race/ethnicity effect, White participants at Ryerson should have provided ratings that were significantly different from POC at Ryerson, but not significantly different from White participants at Iowa State. Truly, the best indication of the race/ethnicity effect is the comparison between White and POC participants at Ryerson, as location is held constant. The constancy in location likely means far greater similarity in social context that between Iowa State participants and either of the Ryerson groups. However, White participants at Ryerson provided responses 
that were not significantly different from either other group, though it is possible that there were not enough White participants at Ryerson to generate the statistical power required to find any significant differences between their ratings and those provided by POC at Ryerson. All in all, although there is some support for the hypothesis that differences across the two samples are related to differences in their racial/ethnic makeup, at best, this provides only a partial explanation for the location effects, given the non-significant differences between the two groups at Ryerson.

A similar critique can be levied against the hypothesis that location effects are due to ingroup bias. The case file that participants read was always set in Seattle to keep the materials entirely consistent across participants. However, the inadvertent consequence of using the same case files was that participants were rating an individual who was more likely to be seen as an ingroup member by Iowans than by Torontonians. This possibility only became clear when significant location effects were observed after all data had been collected. A proper experiment, designed to address this issue, would require both Canadians and Americans who read about a suspect who is either an in-group member or an out-group member, as well as dependent measures included specifically to address this question. Items about similarity and liking may be inadequate to truly capture in-group bias. One key component of the in-group bias is that individuals must feel an attachment to the group (e.g., Taylor \& Doria, 1981). A broader set of dependent variables is needed, then, to explore the possibility of in-group bias.

Conclusions. The results of Experiment 1 suggest the following general conclusions:

1. Whenever differences were observed, ratings were more favourable for the defendant when he provided an alibi compared to when he did not.

2. The moral desirability of the activities described in an alibi showed only complex 
interactive effects. Thus, the effect of this variable may be contingent on or influenced by contextual and individual difference factors.

3. Alibi moral desirability affected non-legal judgments (e.g., liking, similarity between self and the suspect, overall evaluation of suspect's character), generally in the direction that would be expected. The most favourable responses were in the Morally Desirable condition and the least favourable ones were in the Morally Undesirable condition. Interestingly, participants' liking of the suspect mediated the effect between perceptions of alibi moral desirability and ratings of alibi honesty/likelihood of guilt.

4. The pattern of results differed between the Ryerson and Iowa State samples. There is some evidence that this is due to differences in the race/ethnicity of the two samples and indirect evidence that in-group bias may have been operating, though this latter hypothesis must be tested directly.

5. Olson and Wells' (2004) taxonomy was generally supported. Ratings were consistently more favourable for the suspect when there was strong corroborating evidence for the alibi compared to when there was no corroborating evidence. Weak corroborating evidence was sometimes beneficial compared to no evidence.

6. Higher endorsement of Authoritarian values was consistently related to legal judgments that are less favourable for the suspect. Additionally, significant mediation effects were found such that higher scores on the Authoritarianism subscale were associated with harsher judgments about the morality of the alibi behaviour, which were related to less favourable legal judgments.

7. Many participants did not provide an accurate definition of the term "alibi." 


\section{Experiment 2}

\section{Introduction}

The first purpose of Experiment 2 was to investigate the influence of the moral desirability of alibi activities on legal judgments and to determine whether any effects are consistent across suspect race, specifically White versus Indigenous Canadian. As noted earlier, competing theories predicted that a morally undesirable alibi could be the most or the least credible, compared to morally desirable or neutral alibis. Experiment 1 did not provide strong, unequivocal support to either theory. The possible influence of participants' general feelings about White and Indigenous people on legal judgments was also explored. As in the previous experiment, the second and third purposes of Experiment 2 were to replicate previous findings regarding the role of Authoritarianism in legal judgments and the common presence of misconceptions about what the term "alibi" means. Finally, the crime location (Seattle, WA or Vancouver, BC) was manipulated to investigate whether in-group bias was a viable explanation for the differences in results between the Iowa State and Ryerson samples in Experiment 1.

Similar to Experiment 1, participants were given a fictitious police investigation file to read. Alibi moral desirability was manipulated in the same manner as in Experiment 1 (morally desirable, morally undesirable, neutral, no-alibi control). Additionally, the suspects described in the file were either White or First Nations Canadians. Participants responded to a number of questionnaire items, the Authoritarianism-Conservatism-Traditionalism (ACT) scale (Duckitt et al., 2010), and defined the term "alibi." Following this, they were asked to sign a petition supporting the suspect's request for assistance from Legal Aid Washington or Legal Aid British Columbia. This petition was presented as being unrelated to the study. The study was approved by the Research Ethics Board at Ryerson University and complied with the ethical guidelines in 
the Tri-Council Policy Statement.

\section{Method}

Generation of materials and pilot test. The files presented to participants in Experiment 2 were nearly identical to those used in Experiment 1. The key change is that in Experiment 2, the manipulation of the strength of the evidence was replaced by a manipulation of the suspect's racial/ethnic identity. This latter variable is of particular interest, as it has rarely been manipulated in Canada. The evidence strength variable was removed to ensure the design was not overcomplicated. The suspect race manipulation was primarily achieved through showing participants a photograph of either a White or Indigenous man. The photos were provided by Dr. Jeff Pfeifer (see Jackiw, Arbuthnott, Pfeifer, Marcon, \& Meissner, 2008) and all show the forward-facing head and shoulders of a man wearing a dark red shirt, making an ostensibly neutral expression, with a white background. These are similar to what might be shown in a police mugshot, though there is no suspect information board nor height measurement information in the photograph.

First, a set of photographs of Indigenous men was chosen for pilot testing. The chosen faces were of men who appeared to be in their 20 s or 30 s, were making neutral facial expressions, were moderately physically attractive, and were likely to be recognized as Indigenous by most people. Physical attractiveness was taken into account due to a large literature indicating that defendant appearance affects legal decision-making (e.g., Bull \& Rumsey, 1988; Downs \& Lyons, 1991; Efran, 1974; Levelthal \& Krate, 1977; Solomon \& Schopler, 1978; Stewart, 1980). A small selection of friends and family members rated the physical attractiveness, race stereotypicality, perceived aggressiveness, perceived suspiciousness, and perceived trustworthiness of the men in the photographs. They also guessed the individual's 
age and emotional state (happy, sad, neutral, angry, and afraid). Two faces were chosen for use in the study (see Appendix R). Average ratings of aggressiveness, suspiciousness, and trustworthiness were near the midpoint for all photographs and no clear differences were observed between the images. The chosen faces were at or just below the midpoint for attractiveness and were identified as being highly consistent with respondents' stereotypes of how an Indigenous man would look. Finally, most or all of the respondents indicated that the individuals in the two photographs were making neutral facial expressions.

After the two Indigenous faces were selected, a set of White faces that looked similar to the Indigenous faces were pilot tested in the same way. Respondents were also asked to guess whether the individual in the photograph self-identified as White, African American, Latino, First Nations/Native American, or Asian. Again, ratings near or at the midpoint for aggressiveness, suspiciousness, and trustworthiness were observed for most of the faces. Photographs for which there was not a clear consensus that the individual was both White and making a neutral facial expression were excluded. Finally, two White faces were selected that most closely matched the physical attractiveness ratings and the age estimates of the chosen Indigenous faces (see Appendix R).

Participants. Participants $(N=299)$ were recruited through Ryerson's psychology research participation system. They were awarded one course credit for their introductory psychology mark as compensation. Four participants did not consent to have their questionnaires analyzed during the re-consent process, so none of the information they provided was analyzed.

Data from twelve participants were excluded because they provided inaccurate responses to the manipulation check item about the suspect's race. All twelve were in the First Nations suspect condition. Two said that the suspect was White, two said that the suspect was Black, and 
eight chose the "other" option, most of whom wrote that the suspect was Hispanic or Mexican. An additional participant was removed for failing to respond to this item. This left 286 participants in the sample.

The mean age of participants was 20.71 years ( $S D=4.81$ years), and the sample ranged from 17 years to 50 years. The distribution of ages is noticeably skewed to the right. The median age of the sample was 19 years and the most frequently reported age in the sample was 18 years. Although the majority of the sample (76.4\%) was aged 17-21, a noteworthy number of participants were older than this.

The sample comprised 222 women (77.6\%), 59 men (20.6\%), two Queer individuals (0.7\%), and one participant who self-identified as Queer and Trans* (0.3\%). As in Experiment 1, the sample included substantial racial/ethnic diversity (see Table 22). As participants were permitted to choose as many options as they wished, the categories are not mutually exclusive; 18 participants chose more than one category. As with Experiment 1, many participants chose the "other" option. When this occurred, participants generally provided more specific details about their race (e.g., Filipino) or indicated that they were South/Southeast Asian.

Table 22

Participant racelethnicity

\begin{tabular}{lc}
\hline Racial/Ethnic Group & \\
\hline American Indian or Alaska Native & $1(0.3 \%)$ \\
Black or African American & $26(9.1 \%)$ \\
East Asian & $54(18.9 \%)$ \\
First Nations Canadian & $3(1.0 \%)$ \\
Hispanic or Latino/a & $16(5.6 \%)$ \\
Native Hawaiian or Other Pacific Islander & $2(0.7 \%)$ \\
West Asian/Middle Eastern & $34(11.9 \%)$ \\
White & $127(44.4 \%)$ \\
Other & $47(16.4 \%)$ \\
\hline
\end{tabular}


Design. The study employed a 2 (Alibi Provider Racial Group: White, First Nations Canadian) X 4 (Moral Desirability of Alibi Activities: Undesirable, Neutral, Desirable, No Alibi Control) X 2 (Location: Seattle, Vancouver) design, with all variables manipulated between subjects.

Materials. Participants were given a modified version of the police file used in Experiment 1. The case was either set in Seattle, WA, or Vancouver, BC. The file included the same summary report and interrogation transcript as in Experiment 1. In Experiment 2, a suspect information sheet was stapled to the front of the interrogation transcript. This sheet was modeled after the suspect information sheet included in Marion et al. (2011) and Cowan et al. (2015). It included a photograph of the suspect - either White or Indigenous Canadian - along with his name, birthdate, address, race, and a note that the suspect did not have a prior criminal record.

To maximize the ability to compare the results of Experiments 1 and 2, the questionnaire items are largely identical between studies, though the manipulation check items have been updated. Participants were asked to choose whether the suspect was White, Black, First Nations/Aboriginal, or Other, with the final option having a text entry box. Finally, participants were asked to complete a number of thermometer items about members of particular groups on a scale from 0 (cold) to 100 (warm). The two key items were related to participants' feelings towards Caucasian/White People and First Nations/Indigenous People. To obscure the true purpose of these questions, participants were also asked to rate their feelings towards several other identified groups. The order of thermometer items randomized between participants. Due to an error in study administration, the first 83 participants did not view the thermometer items.

New versions of the petition were created for Experiment 2 based on the limitations discussed in Experiment 1 (see Appendix T). The URL and layout were changed. A new, more 
professional-looking photograph was chosen; a header with a logo and non-functional links to start a petition, search the site, and log in were included near the bottom of the page; nonfunctional links to share the petition to social media sites were included; a functional link to a terms of service and privacy policy was added; and a graphical representation of the number of people who have signed the petition was also included. The text of the petition, however, was essentially identical to the text from Experiment 1. Two versions of the petition were added one petitioning Legal Aid Washington and one petitioning Legal Aid British Columbia.

Procedure. As with Experiment 1, participants in sets of 1-4 were invited into a research room at the Psychology Research and Training Centre at Ryerson University. After informed consent was obtained, participants were given a version of the police file, based on random assignment to condition. When they finished reading, the file was removed and they completed the questionnaire on the computer. Following this, they were asked to sign a petition supporting the suspect's application for legal assistance in mounting a defense at trial. During the debriefing process, participants were given a re-consent form that included checkboxes for participants to consent or refuse consent to have a record of their name and participant number and to consent or refuse to consent to allow their questionnaire data to be analyzed. The re-consent was the same form as used at Ryerson in Experiment 1. In all, 37 participants refused to allow their name to be tracked and connected to the petition. Four participants - including one who did not want his/her name tracked — requested that their questionnaires be deleted. Finally, 21 participants (7.3\%) were highly suspicious of the petition and were excluded from all relevant analyses.

\section{Results}

Manipulation checks. Tests of the manipulations were computed for participants who were not in the control condition (details are available in Appendix U). Results suggested that the 
Morally Undesirable and Neutral alibis were consistently rated differently from each other in terms of moral desirability. As well, the Morally Undesirable alibi and actor were rated as negative and the Neutral alibi was rated as neutral. The Morally Desirable alibi was different from the other conditions and rated positively only for ratings of the behaviour, but not for perceptions of the actor. Overall, this manipulation operated generally as anticipated. As in Experiment 1, fewer participants reported having engaged in the Morally Undesirable activity $(13.3 \%)$ than the Neutral $(61.4 \%)$ or Morally Desirable activities (58.1\%).

Participants also indicated whether the suspect was White, Black, First Nations/Aboriginal, or a member of another group. Thirteen participants were excluded for providing incorrect answers or skipping this item, all from the First Nations condition.

Finally, the two photos within each suspect race condition were compared in terms of liking the suspect and general attributions about the suspect's character. No differences were observed for liking, but there was a trend for differences in general attributions for the First Nations photo only. Consequently, a variable that identifies whether the participants were shown the first or second photo was included in all relevant inferential tests, but no interactions between this variable and Suspect Race were identified, suggesting no differences between the photos within each condition.

Aspects of the alibi. The first set of analyses explored the effect of aspects of the alibi. This included comparing the alibis to the control condition and the influence of alibi content and suspect race. The influence of participants' general feelings about White and Indigenous people on their judgments was also investigated.

Alibi versus no-alibi control. One of the primary research questions in this work is whether having an alibi - regardless of its particular qualities or the individual providing it - 
affects an evaluator's judgments. To investigate this question, the responses of participants whose file included an alibi were compared to the responses of participants whose file did not include an alibi (see Table 23). No significant differences were observed between the two groups for participants' ratings of the suspect's honesty statement or participants' confidence in that judgment. However, the strength of the evidence against the suspect was rated as significantly stronger, and there was a nearly significant trend for higher likelihood of guilt ratings when the suspect presented an alibi compared to when he did not.

There was also a nearly significant tendency for a greater number of guilty verdicts to be provided by participants in the Alibi conditions (39.4\%) than in the Control condition (25.4\%), $\chi^{2}(1)=4.17, p=.053, \varphi=.12$. Overall, $36.3 \%$ of participants provided Guilty verdicts.

Additionally, rates of petition-signing were not significantly different between the Alibi conditions (96 signatures; $54.2 \%$ ) and Control participants (29 signatures; 52.7\%), $\chi^{2}(1)=.04, p$ $=.878, \varphi=.01$. Combined, $53.9 \%$ of participants signed the petition. Significant or nearly significant differences were found for half of the comparisons. Where differences were found, participants provided more favourable responses when the suspect did not provide an alibi.

Table 23

t-test results comparing ratings of participants who were shown an alibi and participants who were not shown an alibi

\begin{tabular}{lcccccc}
\hline & Alibi & Control & & & & \\
& $M(S D)$ & $M(S D)$ & $d f$ & $t$ & $p$ & $d$ \\
\hline Suspect honesty* & $4.06(1.33)$ & $4.27(1.68)$ & 85.83 & -.92 & .362 & -0.15 \\
Confidence in suspect honesty & $4.60(1.48)$ & $4.68(1.65)$ & 280 & -.37 & .698 & -0.05 \\
Strength of the evidence & $3.60(1.56)$ & $3.06(1.35)$ & 113.60 & 2.68 & .009 & 0.36 \\
$\quad$ & & & & & \\
$\quad$ against the suspect* & & & & & \\
Likelihood of suspect guilt & $3.89(1.40)$ & $3.51(1.38)$ & 280 & 1.92 & .056 & 0.27 \\
\hline
\end{tabular}

Note. Comparisons denoted with a * violated the assumption of homogeneity of variances.

Reported results are for tests that do not require this assumption to be met. 
The effect of alibi content, alibi provider race, and crime location. To examine the effects of alibi content, alibi provider race, and crime location, a series of 4 (Alibi Content: Morally Desirable, Morally Undesirable, Neutral, Control) X 2 (Suspect Race: White, First Nations Canadian) X 2 (Crime Location: Seattle, Vancouver) X 2 (Photo: First, Second) ANOVAs were computed for most continuous dependent variables, with all variables manipulated between subjects. Some dependent measures were irrelevant for Control participants and were not collected. As noted earlier, the Photo variable is included to ascertain whether participants' responses were consistent across the two photos within each suspect race category. A Photo X Suspect Race interaction would suggest differences between the two photos within at least one category. No other effects of Photo are practically useful or of theoretical interest. Although it would be ideal to include participants' responses to thermometer items assessing their feelings about First Nations and White people in general as a covariate in the analyses, this would result in all data from the 83 participants who were not shown these items to be removed from the analysis. Consequently, these items will be analyzed in a later section.

Additionally, one of the primary aims of this work was to investigate under what conditions an alibi may benefit a suspect and under what conditions it does not, or even harms a suspect's case. It is possible that some alibi narratives are helpful, whereas others are not. Planned analyses compared participants' responses in the Morally Undesirable, Morally Desirable, and Neutral conditions to those of participants in the Control condition, regardless of whether the main effect of Alibi Content was significant in the ANOVA.

Suspect honesty. The only significant effect worthy of note is an interaction between Alibi Content and Suspect Race (see Table 24). To explore the interaction, univariate ANOVAs were computed to examine the effect of Alibi Content for the White and First Nations suspect 
separately. Alibi Content did not affect participants' ratings of statement honesty for the White suspect, $F(3,144)=1.73, p=.463, \eta_{\mathrm{p}}^{2}=.02$, but it did for the First Nations suspect, $F(3,130)=$ $3.70, p=.013, \eta_{\mathrm{p}}^{2}=.08$. Follow-up analyses indicated that the statement of a First Nations suspect was rated as significantly more honest when it described Morally Undesirable activities than when it described Morally Desirable ones (see Table 25 and Figure 5). The other pairwise comparisons were not significant.

Table 24

ANOVA results for participants' ratings of suspect honesty

\begin{tabular}{lcccc}
\hline Effect & $d f$ & $F$ & $p$ & $\eta_{\mathrm{p}}^{2}$ \\
\hline Alibi Content & 3,250 & 1.95 & .122 & .02 \\
Suspect Race & 1,250 & .31 & .577 & $<.01$ \\
Crime Location & 1,250 & .05 & .816 & $<.01$ \\
Photo & 1,250 & 1.67 & .197 & .01 \\
Alibi Content X Suspect Race & 3,250 & 3.26 & .022 & .04 \\
Alibi Content X Crime Location & 3,250 & 1.12 & .342 & .01 \\
Alibi Content X Photo & 3,250 & 2.67 & .048 & .03 \\
Suspect Race X Crime Location & 1,250 & .36 & .547 & $<.01$ \\
Suspect Race X Photo & 1,250 & 1.29 & .258 & .01 \\
Crime Location X Photo & 1,250 & .05 & .831 & $<.01$ \\
Alibi Content X Suspect Race X Crime Location & 3,250 & .76 & .518 & .01 \\
Alibi Content X Suspect Race X Photo & 3,250 & .50 & .686 & .01 \\
Alibi Content X Crime Location X Photo & 3,250 & 1.55 & .201 & .02 \\
Suspect Race X Crime Location X Photo & 1,250 & 1.52 & .218 & .01 \\
Alibi Content X Suspect Race X Crime Location & 3,250 & .70 & .554 & .01 \\
$\quad$ & & & & \\
\hline
\end{tabular}

Table 25

Pairwise comparisons of participants' ratings of statement honesty under different alibi conditions, when the suspect is First Nations

\begin{tabular}{lcccc}
\hline Comparison & $d f$ & $t$ & $p$ & $d$ \\
\hline Undesirable vs. Neutral & 68 & 1.90 & .061 & 0.46 \\
Undesirable vs. Desirable & 68 & 3.56 & .001 & 0.97 \\
Neutral vs. Desirable & 70 & 1.59 & .116 & 0.37 \\
Undesirable vs. Control & 60 & 1.32 & .191 & 0.34 \\
Desirable vs. Control & 62 & -1.67 & .101 & -0.42 \\
Neutral vs. Control & 62 & -.30 & .767 & -0.07 \\
\hline
\end{tabular}


The planned comparisons between the various alibi groups and the Control group did not show any significant differences. Ratings from participants in the Control condition ( $M=4.27$, $S D=1.68)$ were not significantly different than ratings from participants in the Morally Undesirable $(M=4.32, S D=1.39), t(136)=.19, p=848, d=0.03$, Morally Desirable $(M=3.89$, $S D=1.36), t(135)=-1.46, p=.148, d=-0.25$, or Neutral conditions $(M=3.96, S D=1.22)$, $t(112.30)=-1.24, p=.226, d=-0.21$.

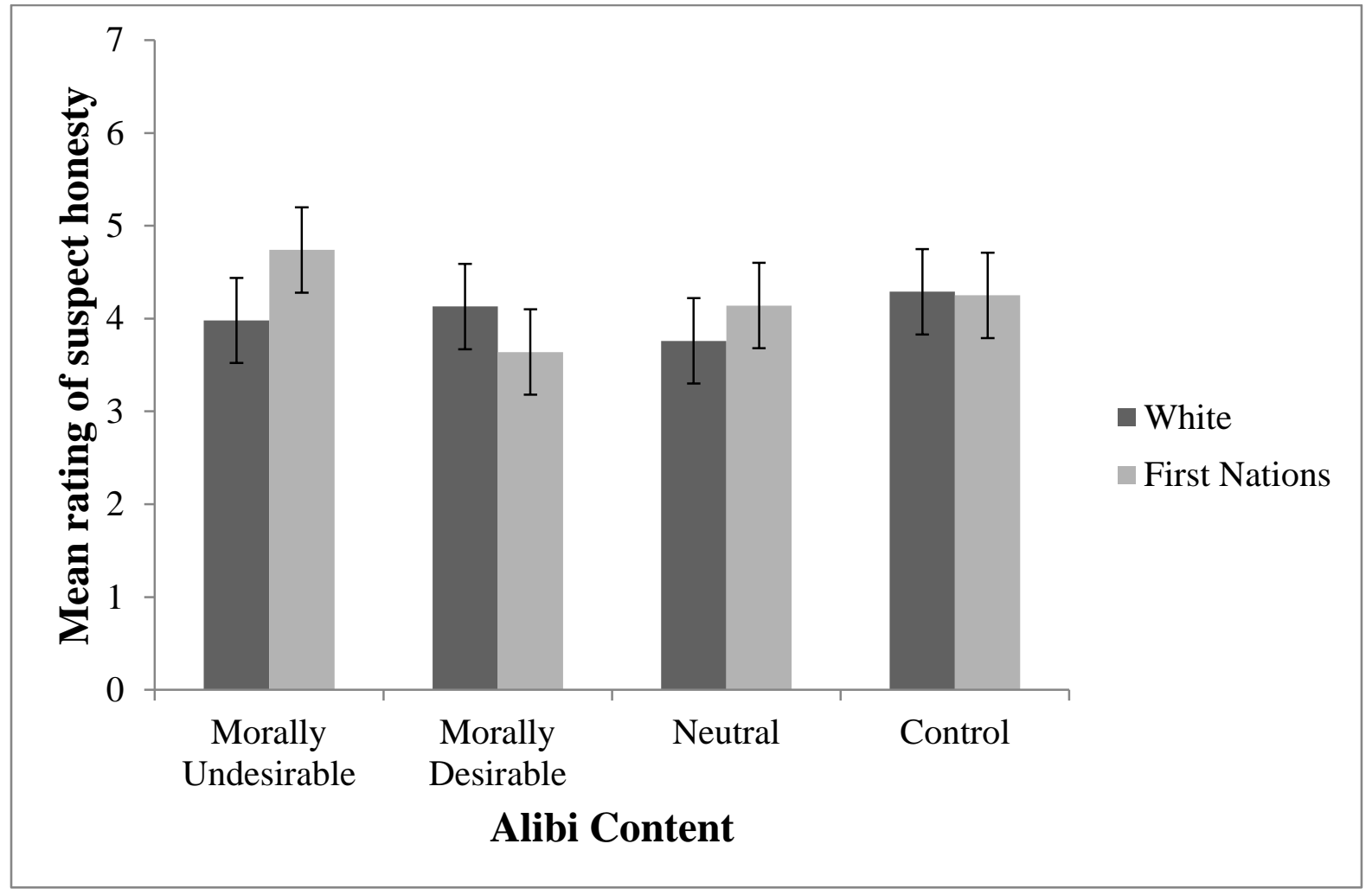

Figure 5. Mean ratings of suspect honesty for White and First Nations suspects for different alibi narratives.

Confidence in suspect honesty. The only significant effect in this analysis is the four-way interaction between Alibi Content, Suspect Race, Crime Location, and Photo (see Table 26). As noted earlier, interactions involving Photo are neither of theoretical nor of practical interest. Given that the three-way interaction between the other variables was not significant, it seems likely that the interaction is spurious. 
However, in the overall analysis, there is a nearly significant interaction between Alibi Content and Crime Location. To examine this interaction, univariate ANOVAs were computed for Alibi Condition separately for participants whose files described a crime set in Seattle and those who read about a crime set in Vancouver. The main effect of Alibi Condition was not significant for the crime in Seattle, $F(3,136)=.68, p=.568, \eta_{p}^{2}=.02$, nor for the crime in Vancouver, $F(3,138)=2.26, p=.084, \eta_{\mathrm{p}}^{2}=.05$.

Table 26

ANOVA results for participants' confidence in their ratings of suspect honesty

\begin{tabular}{lcccc}
\hline Effect & $d f$ & $F$ & $p$ & $\eta_{\mathrm{p}}^{2}$ \\
\hline Alibi Content & 3,250 & .79 & .501 & .01 \\
Suspect Race & 1,250 & 1.58 & .210 & .01 \\
Crime Location & 1,250 & .57 & .353 & $<.01$ \\
Photo & 1,250 & .18 & .674 & $<.01$ \\
Alibi Content X Suspect Race & 3,250 & 1.63 & .182 & .02 \\
Alibi Content X Crime Location & 3,250 & 2.35 & .073 & .03 \\
Alibi Content X Photo & 3,250 & 1.06 & .366 & .01 \\
Suspect Race X Crime Location & 1,250 & 1.08 & .301 & $<.01$ \\
Suspect Race X Photo & 1,250 & .02 & .880 & $<.01$ \\
Crime Location X Photo & 1,250 & 2.15 & .14 & .01 \\
Alibi Content X Suspect Race X Crime Location & 3,250 & .61 & .611 & .01 \\
Alibi Content X Suspect Race X Photo & 3,250 & 2.27 & .081 & .03 \\
Alibi Content X Crime Location X Photo & 3,250 & .72 & .543 & .01 \\
Suspect Race X Crime Location X Photo & 1,250 & 1.78 & .184 & .01 \\
Alibi Content X Suspect Race X Crime Location & 3,250 & 3.57 & .015 & .04 \\
$\quad$ X Photo & & & & \\
\hline
\end{tabular}

For the planned comparisons between the Control participants and participants in each of the other three Alibi Content conditions, no significant effects were observed. Confidence of the Control participants $(M=4.68, S D=1.65)$ was not significantly different than the confidence of the Morally Undesirable participants $(M=4.47, S D=1.47), t(136)=-.81, p=.419, d=-0.13$, the Morally Desirable participants $(M=4.55, S D=1.57), t(135)=-.47, p=.642, d=-0.08$, or the Neutral participants $(M=4.79, S D=1.39), t(131)=.39, p=.697, d=0.07$. 
Alibi accuracy. The only significant effects for alibi accuracy ratings were interactions between Alibi Content and Suspect Race and between Suspect Race and Crime Location (see Table 27). As participants in the Control group did not respond to this item, they were not included in the analysis. To explore the Alibi Content X Suspect Race interaction, ANOVAs were computed to examine the effect of Alibi Content for the White and First Nations suspects separately (see Figure 6). For the White suspect, there was no significant effect of Alibi Content, $F(2,110)=.74, p=.479, \eta_{\mathrm{p}}^{2}=.01$. However a significant main effect was found for the First Nations suspect, $F(2,104)=3.66, p=.029, \eta_{\mathrm{p}}^{2}=.07$. Follow-up independent-samples $t$-tests showed that an alibi provided by a First Nations suspect was rated as significantly more accurate when it was Morally Undesirable than Morally Desirable, $t(68)=2.68, p=.009, d=0.64$. The Morally Undesirable alibi provided by the First Nations suspect was also rated as marginally more accurate than when the alibi was Neutral, $t(69)=2.00, p=.049, d=0.48$. No significant differences were observed between First Nations suspects with Morally Desirable or Neutral alibis, $t(71)=-.41, p=.681, d=-0.09$.

To examine the interaction between Suspect Race and Crime Location, participants' ratings of alibi accuracy for the White and First Nations suspect were compared separately for the files set in Seattle and Vancouver. When the crime was set in Seattle, there were no significant differences for participants' ratings of the accuracy of the alibi provided by White ( $M$ $=3.72, S D=1.24)$ or First Nations suspects $(M=4.16, S D=1.30), t(109)=-1.82, p=.071, d=-$ 0.35. When the crime was set in Vancouver, there were also no significant differences between ratings for the White $(M=4.09, S D=1.55)$ and First Nations suspects $(M=3.75, S D=1.21)$, $t(98.42)=1.29, p=.200, d=0.25$. The significant interaction is likely due to these opposite - if non-significant - patterns; when the crime was set in Seattle, participants' ratings of alibi 
Table 27

ANOVA results for participants' ratings of alibi accuracy

\begin{tabular}{lcccc}
\hline Effect & $d f$ & $F$ & $p$ & $\eta_{\mathrm{p}}^{2}$ \\
\hline Alibi Content & 2,196 & 2.28 & .106 & .02 \\
Suspect Race & 1,196 & .12 & .729 & $<.01$ \\
Crime Location & 1,196 & $<.01$ & .972 & $<.01$ \\
Photo & 1,196 & .22 & .636 & $<.01$ \\
Alibi Content X Suspect Race & 2,196 & 3.07 & .048 & .03 \\
Alibi Content X Crime Location & 2,196 & .92 & .402 & .01 \\
Alibi Content X Photo & 2,196 & 2.06 & .130 & .02 \\
Suspect Race X Crime Location & 1,196 & 4.36 & .038 & .02 \\
Suspect Race X Photo & 1,196 & .02 & .876 & $<.01$ \\
Crime Location X Photo & 1,196 & 2.67 & .104 & .01 \\
Alibi Content X Suspect Race X Crime Location & 2,196 & 1.09 & .340 & .01 \\
Alibi Content X Suspect Race X Photo & 2,196 & .47 & .629 & .01 \\
Alibi Content X Crime Location X Photo & 2,196 & .87 & .420 & .01 \\
Suspect Race X Crime Location X Photo & 1,196 & .87 & .352 & $<.01$ \\
Alibi Content X Suspect Race X Crime Location & 2,196 & 1.52 & .222 & .02 \\
$\quad$ & & & & \\
\hline \multicolumn{1}{c}{ X Photo } & & & & \\
\hline
\end{tabular}

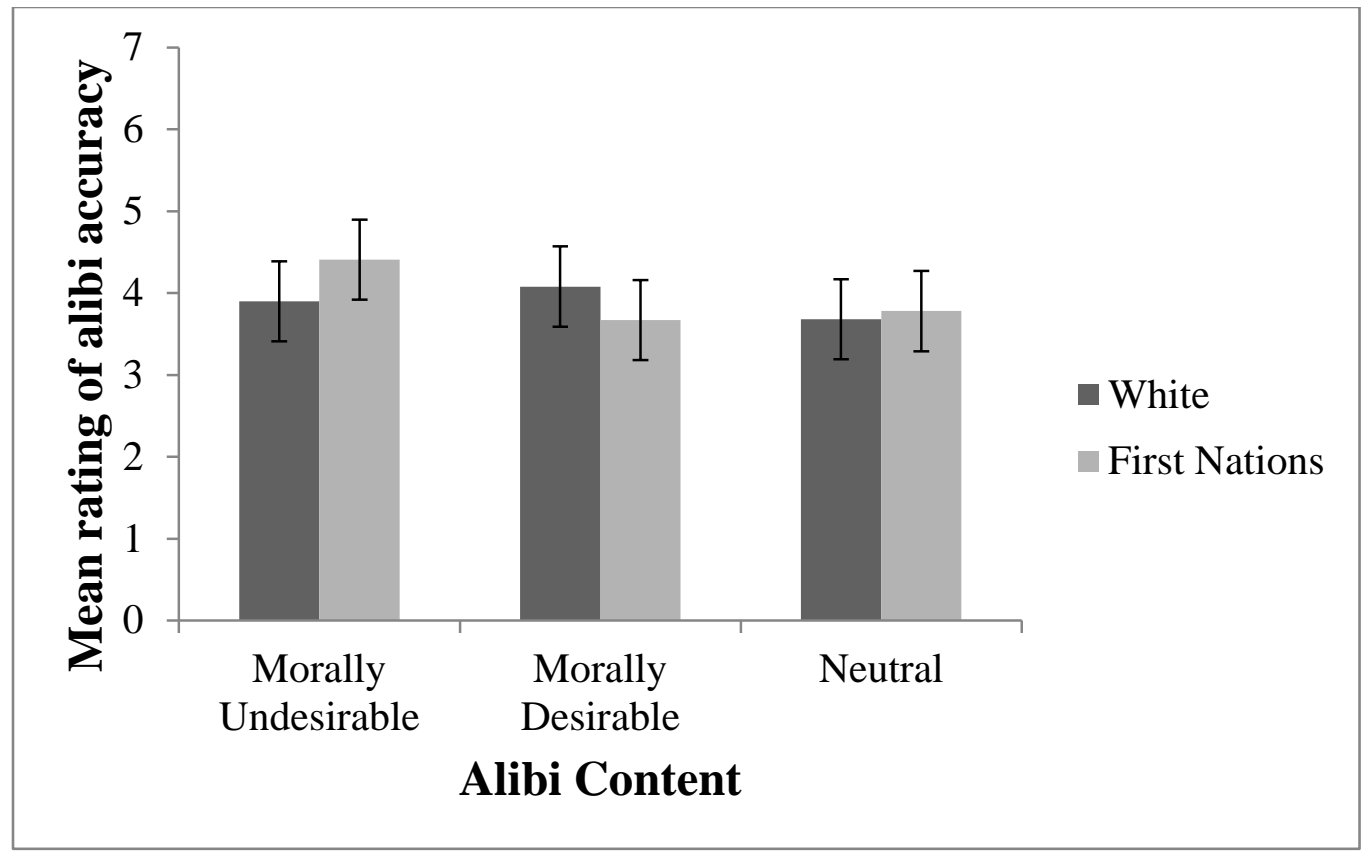

Figure 6. Mean ratings of suspect honesty for White and First Nations suspects for different alibi narratives. 
accuracy were higher for the First Nations suspect, but when the crime was set in Vancouver, ratings were higher for the White suspect.

Confidence in ratings of alibi accuracy. Participants who were not in the Control group were also asked to rate their confidence in their judgment about the accuracy of the alibi. Analyses show only one significant effect: the interaction between Alibi Content, Suspect Race, and Photo (see Table 28). This interaction is not of theoretical or practical interest, though the two-way interaction between Alibi Content and Suspect Race is marginally significant. To examine this marginal interaction, the effect of Alibi Content was examined separately for White and First Nations suspects using ANOVAs (see Figure 7). The main effect for Alibi Content was not significant for the White suspect, $F(2,109)=.29, p=.746, \eta_{\mathrm{p}}^{2}=.01$, nor for the First Nations suspect, $F(2,104)=2.39, p=.097, \eta_{\mathrm{p}}^{2}=.04$.

Table 28

ANOVA results for participants' confidence in their ratings of alibi accuracy

\begin{tabular}{lcccc}
\hline Effect & $d f$ & $F$ & $p$ & $\eta_{\mathrm{p}}^{2}$ \\
\hline Alibi Content & 2,195 & .85 & .428 & .01 \\
Suspect Race & 1,195 & 2.18 & .142 & .01 \\
Crime Location & 1,195 & $<.01$ & .959 & $<.01$ \\
Photo & 1,195 & .94 & .335 & .01 \\
Alibi Content X Suspect Race & 2,195 & 2.70 & .070 & .03 \\
Alibi Content X Crime Location & 2,195 & .07 & .932 & $<.01$ \\
Alibi Content X Photo & 2,195 & .74 & .478 & .01 \\
Suspect Race X Crime Location & 1,195 & .80 & .372 & $<.01$ \\
Suspect Race X Photo & 1,195 & .16 & .690 & $<.01$ \\
Crime Location X Photo & 1,195 & .25 & .618 & $<.01$ \\
Alibi Content X Suspect Race X Crime Location & 2,195 & 1.42 & .246 & .01 \\
Alibi Content X Suspect Race X Photo & 2,195 & 3.23 & .042 & .03 \\
Alibi Content X Crime Location X Photo & 2,195 & 1.26 & .287 & .01 \\
Suspect Race X Crime Location X Photo & 1,195 & .41 & .521 & $<.01$ \\
Alibi Content X Suspect Race X Crime Location & 3,195 & .78 & .462 & .01 \\
$\quad$ X Photo & & & & \\
\hline
\end{tabular}




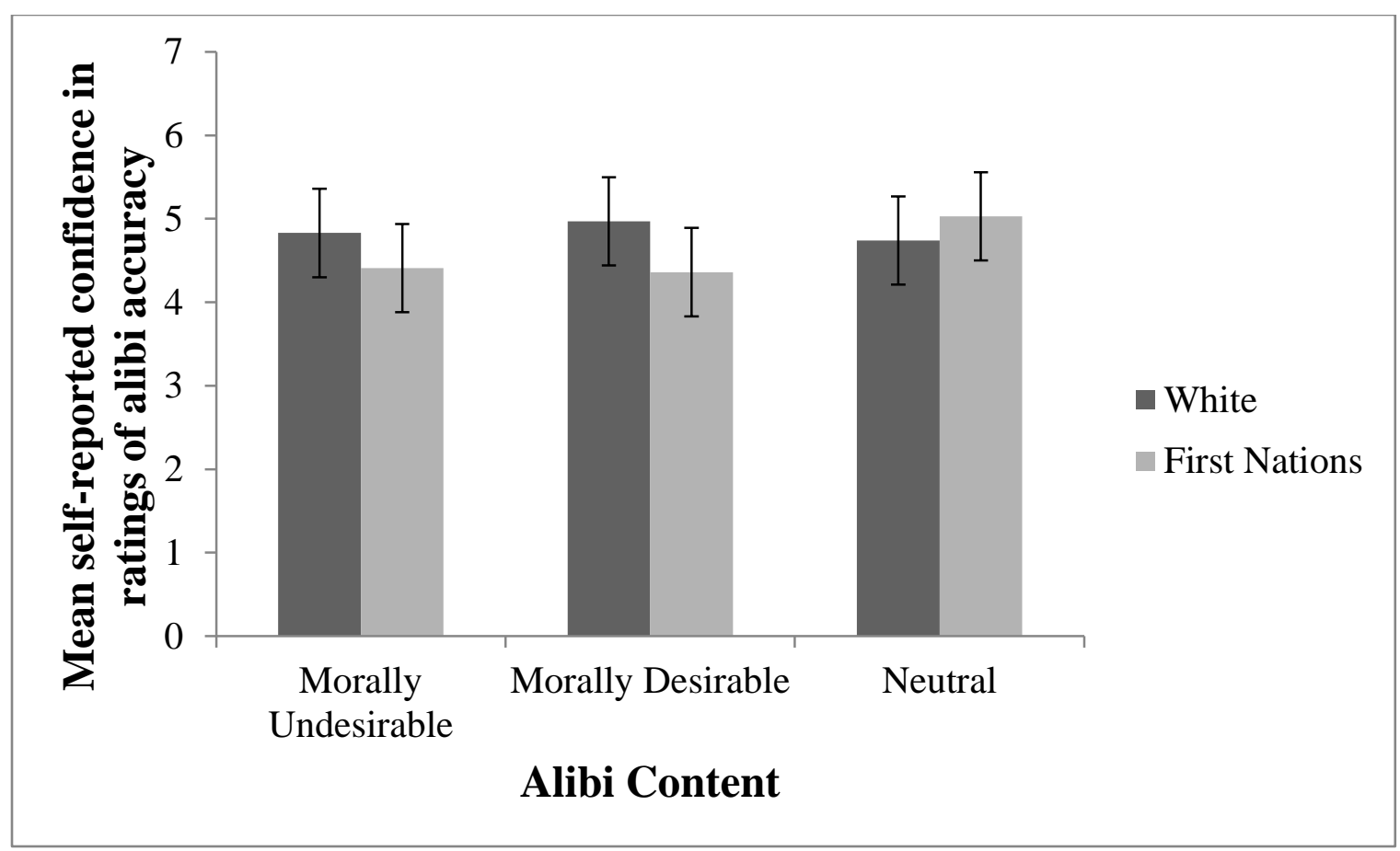

Figure 7. Mean self-reported confidence of participants' ratings of suspect honesty for White and First Nations suspects for different alibi narratives.

Strength of the evidence against the suspect. Participants' ratings of the strength of the evidence against the suspect were significantly affected by Alibi Content alone (see Table 29). Although there was a significant three-way interaction, this effect included Photo, and the twoway interaction between the other variables was non-significant. A series of independentsamples $t$-tests were computed to investigate the main effect of Alibi Content (see Table 30 and Figure 8). In general, participants rated the evidence against the suspect as moderately strong. With six comparisons, the only significant difference was that the evidence against the suspect was rated as stronger in the Desirable condition than in the Control condition. However, if only the three planned comparisons between the alibi conditions and the Control condition are considered, the strength of the evidence against the suspect is also rated as higher in the Neutral condition than in the Control condition. Evidence that suggests that a suspect is guilty may be rated as stronger when s/he offers an alibi - at least when the alibi describes morally desirable or 
neutral activities - compared to when the suspect does not offer an alibi. This is the case despite there being no manipulations of the strength of the incriminating evidence between the various versions of the case file.

Table 29

ANOVA results for participants' ratings of the strength of the evidence against the suspect

\begin{tabular}{lcccc}
\hline Effect & $d f$ & $F$ & $p$ & $\eta_{\mathrm{p}}^{2}$ \\
\hline Alibi Content & 3,251 & 2.73 & .045 & .03 \\
Suspect Race & 1,251 & .83 & .364 & $<.01$ \\
Crime Location & 1,251 & .10 & .756 & $<.01$ \\
Photo & 1,251 & .03 & .867 & $<.01$ \\
Alibi Content X Suspect Race & 3,251 & 1.26 & .289 & .02 \\
Alibi Content X Crime Location & 3,251 & .83 & .477 & .01 \\
Alibi Content X Photo & 3,251 & .53 & .661 & .01 \\
Suspect Race X Crime Location & 1,251 & .01 & .931 & $<.01$ \\
Suspect Race X Photo & 1,251 & .87 & .352 & $<.01$ \\
Crime Location X Photo & 1,251 & 1.69 & .195 & .01 \\
Alibi Content X Suspect Race X Crime Location & 3,251 & .23 & .879 & $<.01$ \\
Alibi Content X Suspect Race X Photo & 3,251 & 2.66 & .049 & .03 \\
Alibi Content X Crime Location X Photo & 3,251 & .83 & .476 & .01 \\
Suspect Race X Crime Location X Photo & 1,251 & .03 & .857 & $<.01$ \\
Alibi Content X Suspect Race X Crime Location & 3,251 & .35 & .793 & $<.01$ \\
$\quad$ X Photo & & & & \\
\hline
\end{tabular}

Table 30

Pairwise comparisons of participants' ratings of the strength of the evidence against the suspect under different alibi conditions

\begin{tabular}{lcccc}
\hline Comparison & $d f$ & $t$ & $p$ & $d$ \\
\hline Undesirable vs. Neutral & 144 & -1.85 & .067 & -0.30 \\
Undesirable vs. Desirable & 147 & -1.23 & .256 & -0.20 \\
Neutral vs. Desirable & 143 & .52 & .601 & 0.09 \\
Undesirable vs. Control & 136 & 1.18 & .239 & 0.21 \\
Desirable vs. Control* & 134.61 & 2.31 & .023 & 0.39 \\
Neutral vs. Control & 132 & 2.93 & .004 & 0.51 \\
\hline
\end{tabular}

Note. Comparisons marked with a * did not meet the assumption of equality of variances, and the test that does not require this assumption is reported. 


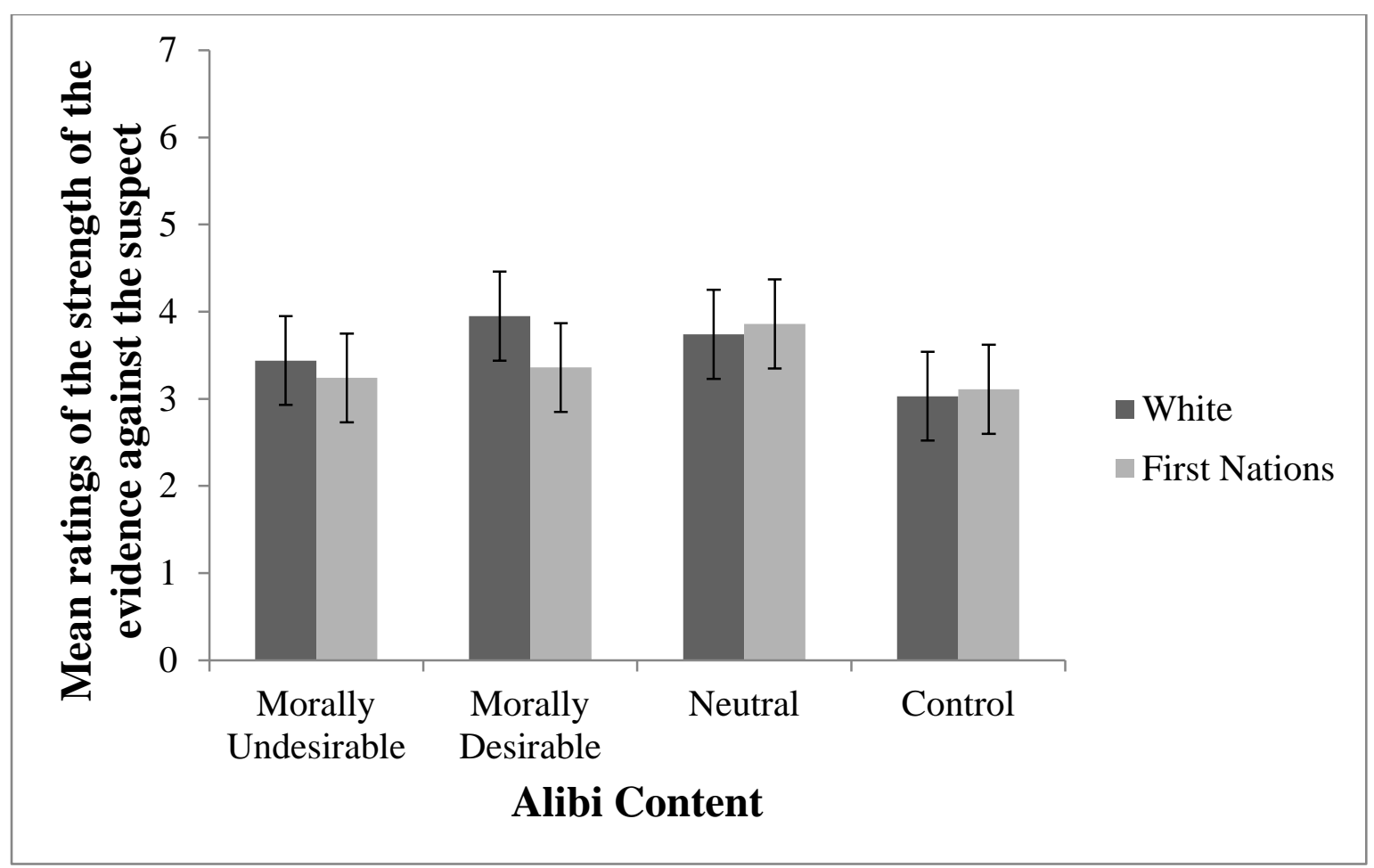

Figure 8. Participants' ratings of the strength of the evidence against the suspect for White and First Nations suspects who offered different alibis.

Alibi classification. Participants who read the suspect's alibi were also asked to judge whether that alibi was True (i.e., honest and accurate), Fabricated (i.e., dishonest and inaccurate), or Mistaken (i.e., honest and inaccurate). Participants most frequently decided that the alibi was truthful $(43.1 \%)$ or fabricated $(37.2 \%)$, but this time a substantial number of participants indicated that the alibi was mistaken $(19.7 \%)$. This is the same pattern of responses as was observed in Experiment 1, though the exact rates did vary (True: 58.8\%; Fabricated: 27.0\%; Mistaken: $14.2 \%)$.

To determine whether Alibi Content, Suspect Race, or Crime Location influenced participants' classification of the alibi, a multinomial logistic regression was computed with all the independent variables - including Photo - included as factors. As in Experiment 1, the Neutral condition was chosen as the reference category for Alibi Content, as it represents the 
absence of the independent variable in question (i.e., moral desirability), and Fabricated was chosen as the reference category for the outcome variable because the comparison between True and Mistaken was not of primary theoretical interest. In the initial analysis, two cells included zero frequencies, and initial results indicated no significant effects of Photo. Consequently, the analysis was re-computed with this variable removed, and all cells contained non-zero values.

Non-significant Pearson, $\chi^{2}(14)=20.77, p=.108$, and deviance, $\chi^{2}(14)=21.20, p=.097$, values in the goodness-of-fit tests suggest that the model is a good fit for the data. However, the model fit test was also non-significant, $\chi^{2}(8)=7.75, p=.458$, indicating that the final model does not fit the data significantly better than does the intercept-only model. This is consistent with the results of the two logits (i.e., Truth vs. Fabricated and Mistaken vs. Fabricated); no significant effects were observed for any predictor in either logit (all $p>.092)$.

Likelihood of suspect guilt. No significant meaningful effects were found for participants' ratings of the likelihood that the suspect was guilty (see Table 31) and no significant differences were found in the planned comparisons between each alibi group and the Control group. Likelihood of guilt ratings were the lowest in the Control condition $(M=3.51, S D=1.39)$. Although they were not significantly lower than ratings provided by the Morally Undesirable $(M$ $=3.73, S D=1.32), t(136)=.98, p=.329, d=0.16$, Morally Desirable $(M=3.92, S D=1.41)$, $t(134)=1.71, p=.090, d=0.29$, or Neutral $(M=4.03, S D=1.38), t(132)=2.11, p=.037, d=$ 0.37, groups, the latter two comparisons trended towards significance. As with participants' ratings of the strength of the evidence against the suspect, there is clearly no difference between the responses of the Control group and the Morally Undesirable group. Again, providing a Morally Desirable or Neutral alibi leads to less favourable ratings for the suspect compared to providing no alibi. The mean ratings for likelihood of guilt were at or below the midpoint of 4, 
suggesting that participants were relatively uncertain about whether the suspect was guilty or innocent.

Table 31

ANOVA results for participants' ratings of the likelihood that the suspect is guilty

\begin{tabular}{lcccc}
\hline Effect & $d f$ & $F$ & $p$ & $\eta_{\mathrm{p}}^{2}$ \\
\hline Alibi Content & 3,250 & 1.68 & .173 & .02 \\
Suspect Race & 1,250 & .20 & .658 & $<.01$ \\
Crime Location & 1,250 & .15 & .703 & $<.01$ \\
Photo & 1,250 & 1.00 & .319 & $<.01$ \\
Alibi Content X Suspect Race & 3,250 & 1.5 & .215 & .02 \\
Alibi Content X Crime Location & 3,250 & .22 & .881 & $<.01$ \\
Alibi Content X Photo & 3,250 & 2.17 & .093 & .03 \\
Suspect Race X Crime Location & 1,250 & 1.39 & .239 & .01 \\
Suspect Race X Photo & 1,250 & .01 & .917 & $<.01$ \\
Crime Location X Photo & 1,250 & .01 & .920 & $<.01$ \\
Alibi Content X Suspect Race X Crime Location & 3,250 & .40 & .757 & .01 \\
Alibi Content X Suspect Race X Photo & 3,250 & .79 & .501 & .01 \\
Alibi Content X Crime Location X Photo & 3,250 & .27 & .849 & $<.01$ \\
Suspect Race X Crime Location X Photo & 1,250 & .11 & .744 & $<.001$ \\
Alibi Content X Suspect Race X Crime Location & 3,250 & 2.51 & .059 & .03 \\
$\quad$ & & & & \\
\hline
\end{tabular}

Verdict choice. Overall, $36.3 \%$ of participants provided guilty verdicts. A loglinear analysis with backwards elimination was computed to identify whether Alibi Content, Suspect Race, Crime Location, and Photo were significantly associated with Verdict Choice. Although there are sufficient degrees of freedom in the error term to justify the use of ANOVA, cell sizes are not equal in these data, so ANOVA is not appropriate. In the initial analysis, 50\% of expected cell counts were below five. Results from loglinear analysis are only reliable when fewer than $20 \%$ of expected cell counts do not meet this threshold. A separate analysis with only Suspect Race, Photo, and Verdict Choice did not retain the three-way interaction, suggesting that Photo could be safely removed from the analysis. The goodness-of-fit test of the likelihood ratio on that analysis, $\chi^{2}(6)=6.34, p=.386$, suggested that the model was a good fit for the data. 
In the loglinear analysis that included Alibi Content, Suspect Race, Crime Location, and Verdict Choice, only $12.5 \%$ of cells had expected counts less than five, making the results reliable. The goodness-of-fit test on the likelihood ratio showed a good fit of the model to the data, $\chi^{2}(24)=16.37, p=.874$. An interaction between Alibi Content and Verdict Choice was the only effect that remained in the model. To examine this interaction, chi-square analyses were computed to compare each level of Alibi Content to each other level in terms of the number of guilty verdicts generated (see Table 32). None of the comparisons were significant with the Holm-Bonferroni correcting for six analyses, although three comparisons showed $p$-values below .05 .

Table 32

Pairwise comparison of guilty verdict rates for different alibi conditions

\begin{tabular}{lcccc}
\hline Comparison & $d f$ & $\chi^{2}$ & $p$ & $\varphi$ \\
\hline Undesirable vs. Neutral & 1 & 2.47 & .123 & -.13 \\
Undesirable vs. Desirable & 1 & 4.42 & .042 & -.17 \\
Neutral vs. Desirable & 1 & .27 & .619 & .04 \\
Undesirable vs. Control & 1 & .32 & .702 & .05 \\
Desirable vs. Control & 1 & 6.52 & .013 & .22 \\
Neutral vs. Control & 1 & 4.21 & .046 & -.18 \\
\hline
\end{tabular}

As noted earlier, analyses were planned to compare each of the alibi conditions to the control. When only these are considered, however, significant differences emerge. The number of guilty verdicts did not vary between the Control (25.4\%) and Morally Undesirable (29.7\%) conditions. However, there was a significantly higher rate of guilty verdicts in the Morally Desirable condition (46.6\%), than in the Control condition. Although the difference was not statistically significant, there was a trend towards a greater number of guilty verdicts in the Neutral condition (42.3\%) than in the Control condition. As with earlier analyses, the observed differences between the Control and alibi groups are driven by responses that are less favourable 
to the suspect when the alibi is Morally Desirable, and, possibly, when the alibi is Neutral.

Petition signing. Across the sample, $53.9 \%$ of participants signed the petition. This is nearly identical to the rate of petition signing observed in Experiment 1 (52.8\%). A loglinear analysis with backwards elimination was computed to determine whether participants' willingness to sign the petition was affected by Alibi Content, Suspect Race, Crime Location, and Photo. As with the loglinear analysis on Verdict Choice, a high number of expected cell counts $(68.75 \%)$ fell below the five-count cutoff, indicating that the results of the analysis are not reliable. As in the previous section, a follow-up analysis that only included Suspect Race, Photo, and Petition Signing showed no three-way interaction, and good fit, $\chi^{2}(4)=3.63, p=.458$, suggesting Photo can be safely removed. Unfortunately, the loglinear analysis that included Alibi Content, Suspect Race, Crime Location, and Petition Signing had 25\% of expected cell counts below five, which remains too high to consider results reliable. The same problem emerges when the loglinear analysis is computed separately based on whether the crime was set in Seattle or Vancouver, or based on whether the suspect is White or First Nations. However, it is still possible to compute chi-square analyses examining the effect of each independent variable on Petition Signing separately.

To examine whether participants' willingness to sign the petition in support of the suspect was influenced by Alibi Content, a chi-square test was computed on these variables. The test was not significant, $\chi^{2}(3)=3.28, p=.352, V=.12$. As well, in the planned comparisons, rates of petition signing were equivalent between the Control condition (52.7\%) and the Morally Undesirable $(48.4 \%), \chi^{2}(1)=.22, p=.712, \varphi=-.04$, Morally Desirable $(50.9 \%), \chi^{2}(1)=.04, p=$ $.853, \varphi=-.02$, or Neutral conditions $(63.8 \%), \chi^{2}(1)=1.42, p=.257, \varphi=.11$.

A chi-square analysis was also computed to examine whether petition signing was 
significantly associated to Suspect Race. The test showed a significant effect, $\chi^{2}(1)=4.25, p=$ $.048, \varphi=-.14$. Participants were significantly more likely to sign the petition when the suspect was First Nations (61.1\%) than when the suspect was White (47.6\%).

Finally, a chi-square analysis was computed to determine whether petition signing was related to Crime Location. Interestingly, a significant effect was observed, $\chi^{2}(1)=.6 .68, p=$ $.012, \varphi=-.17$. Consistent with what would be expected if in-group bias was operating, participants were significantly more likely to sign the petition when the crime was set in Vancouver (62.0\%) than when it was set in Seattle (45.0\%).

To determine whether there was an interaction between Suspect Race and Crime Location, a final loglinear analysis with these two variables and Petition Signing was computed. All expected cell counts were well above five, and the chi-square test on the likelihood ratio suggests that the model is a good fit to the data, $\chi^{2}(2)=1.65, p=.439$. The three-way effect was removed from the model, meaning that there was no significant interaction between Suspect Race and Crime Location. However, significant interactions between Crime Location and Petition Signing and Suspect Race and Petition Signing remained in the model, consistent with the results of the chi-square analyses in the previous paragraphs. Overall, the results show that participants are more likely to sign a petition supporting the suspect when he is a First Nations person and when the crime is set in Vancouver.

Participant Race/Ethnicity. The results of Experiment 1 showed some differences between the responses of White participants at Iowa State and People of Colour (POC) at Ryerson, with the former providing responses that were more favourable to the suspect. 2 (Participant Race/Ethnicity: White, POC) X 2 (Suspect Race: White, First Nations) were computed (see Appendix V). Participants' race/ethnicity was not significantly related to attitude 
measures, but White participants $(64.2 \%)$ were more likely to sign the petition than were POC $(53.9 \%)$.

The influence of participants' general feelings about First Nations and White people on their legal judgments. Participants indicated their feelings about a number of identifiable groups of people, including White and First Nations/Indigenous people. As noted earlier, due to an error in study administration, the first 83 participants were not shown these items. Four participants did not respond to either of these items, and two provided responses only for their perceptions of White people. Detailed results are provided in Appendix W. Briefly, participants' responses to both items were generally very positive. The correlation between their ratings was moderate-to-high. Regression models computed separately for First Nations and White suspects. For each, an initial simple regression was computed with only the relevant attitude included as a predictor (e.g., attitudes about White people predicting responses to a White suspect), and a second regression was computed that also controlled for the other attitude (e.g., including attitudes about First Nations as a predictor in the earlier example). Results did not provide strong evidence that participants' general attitudes about White or First Nations people were related to their judgments, though this may have been due to restriction of range in the predictors. However, having relatively more positive feelings about White people was associated with a greater likelihood of signing the petition for the White suspect, even when attitudes about First Nations people were controlled. No similar pattern was observed for signing the petition when the suspect was First Nations.

Evidence for implicit personality theories. One theory presented in the introduction suggested that participants form implicit personality theories based on positive or negative alibi information, and that these influence their legal judgments. Participants' perceptions of the 
similarity between themselves and the suspect, their liking for the suspect, and their overall evaluation of the suspect's character are indications of participants' overall assessment of the suspect. To explore whether the Alibi Moral Desirability manipulation affected participants' broad impressions of the suspect, univariate ANOVAs were computed with Alibi Content as the independent variable and participants' ratings of the similarity between themselves and the suspect, of how much they like the suspect, and of whether they thought the suspect was a good or bad person as dependent variables. Other analyses were computed that included Alibi Moral Desirability, Crime Location, and Suspect Race, but the latter two variables showed no significant main or interactive effects, so the results of analyses that only included Alibi Moral Desirability as an independent variable are reported. It was hypothesized that participants would like the suspect more, think that they are more similar to him, and believe he is a better person when the alibi described Morally Desirable behaviour relative to the other conditions.

Participants' ratings of how similar they are to the suspect in terms of personality were significantly affected by Alibi Condition, $F(3,277)=4.77, p=.003, \eta_{\mathrm{p}}^{2}=.049$ (see Tables 33 34). Participants thought they were significantly less similar to the suspect when his alibi described morally undesirable activities than when the alibi described morally desirable activities, or when the suspect did not offer an alibi (i.e., Control condition). There was also a trend for participants to rate themselves as significantly less similar than the suspect with the Morally Undesirable alibi than the Neutral alibi. It is worth noting that all groups rated themselves as fairly dissimilar to the suspect, with the highest mean being 2.43 on a 7-point scale. It seems that participants who read the Morally Undesirable alibi believed that they were particularly dissimilar to the suspect. 
Table 33

Descriptive statistics for participants' ratings of similarity, liking, and whether the suspect is a good or bad person, separated by Alibi Content group

\begin{tabular}{llccc}
\hline Outcome & Alibi Condition & $M$ & $S D$ & $n$ \\
\hline Similarity & Morally Undesirable & 1.67 & .99 & 75 \\
& Neutral & 2.07 & 1.07 & 70 \\
& Morally Desirable & 2.19 & 1.39 & 73 \\
& Control & 2.43 & 1.41 & 63 \\
Liking & & & \\
& Morally Undesirable & 2.35 & 1.12 & 75 \\
& Neutral & 2.94 & 1.27 & 70 \\
& Morally Desirable & 3.01 & 1.38 & 72 \\
& Control & 3.24 & 1.10 & 63 \\
Is suspect a bad or & & & & \\
bad person & Morally Undesirable & 5.01 & 1.43 & 75 \\
& Neutral & 5.61 & 1.68 & 70 \\
& Morally Desirable & 5.89 & 1.41 & 73 \\
& Control & 5.84 & 1.17 & 63 \\
\hline
\end{tabular}

Note. Similarity and Liking were rated on a 1-7 scale and overall evaluations of the suspect's character were rated on a 1-11 scale.

In terms of liking the suspect, group means show that the participants generally disliked the suspect. A significant effect was found for Alibi Condition, $F(3,276)=6.86, p<.001, \eta_{\mathrm{p}}^{2}=$ .069. Follow-up independent-samples $t$-tests showed that participants liked the suspect significantly less with the Morally Undesirable alibi than in the other alibi conditions, which did not differ among themselves (see Table 34).

For participants' overall evaluation of the suspect's character, group means were all near or below the midpoint, suggesting that the participants generally had neutral to mildly negative views of the suspect. Participants' impressions of the suspect's overall character were affected by Alibi Content, $F(3,277)=5.72, p=.001, \eta_{\mathrm{p}}^{2}=.058$. Participants' ratings were significantly more negative in the Morally Undesirable and Control conditions than in the Morally Desirable 
condition (see Table 34). There was a trend for the suspect to be rated more negatively in the Morally Undesirable condition than in the Neutral condition. Results in this section suggest that participants who were exposed to a Morally Undesirable alibi formed a more negative view of the suspect than individuals in the other conditions. However, there was no evidence of positive implicit personality theories in response to the Morally Desirable alibi.

Table 34

Pairwise comparison of different levels of alibi moral desirability on similarity ratings

\begin{tabular}{|c|c|c|c|c|c|}
\hline Outcome & Comparison & $d f$ & $t$ & $p$ & $d$ \\
\hline \multicolumn{6}{|l|}{ Similarity } \\
\hline & Morally Undesirable - Neutral & 143 & -2.37 & .019 & -0.39 \\
\hline & Morally Undesirable - Morally Desirable* & 129.87 & -2.64 & .009 & -0.43 \\
\hline & Morally Undesirable - Control* & 108.43 & -3.60 & $<.001$ & -0.63 \\
\hline & Neutral - Morally Desirable & 141 & -.58 & .564 & -0.10 \\
\hline & Neutral - Control* & 114.93 & -1.63 & .105 & -0.29 \\
\hline & Morally Desirable - Control & 134 & -.98 & .327 & -0.17 \\
\hline \multicolumn{6}{|l|}{ Liking } \\
\hline & Morally Undesirable - Neutral & 143 & -3.00 & .003 & -0.49 \\
\hline & Morally Undesirable - Morally Desirable & 145 & -3.23 & .002 & -0.53 \\
\hline & Morally Undesirable - Control & 136 & -4.69 & $<.001$ & -0.80 \\
\hline & Neutral - Morally Desirable & 140 & -.32 & .750 & -0.05 \\
\hline & Neutral - Control & 131 & -1.42 & .157 & -0.25 \\
\hline & Morally Desirable - Control & 133 & -1.03 & .303 & -0.18 \\
\hline \multicolumn{6}{|c|}{$\begin{array}{l}\text { Is suspect a bad } \\
\text { or good person }\end{array}$} \\
\hline & Morally Undesirable - Neutral & 143 & -2.33 & .021 & -0.39 \\
\hline & Morally Undesirable - Morally Desirable & 146 & -3.76 & $<.001$ & -0.88 \\
\hline & Morally Undesirable - Control & 134 & .22 & .827 & 0.04 \\
\hline & Neutral - Morally Desirable & 141 & -1.07 & .288 & -0.18 \\
\hline & Neutral - Control* & 123.37 & -.90 & .372 & -0.16 \\
\hline & Morally Desirable - Control* & 135.91 & -3.75 & $<.001$ & -0.63 \\
\hline
\end{tabular}

Note. Comparisons marked with a * did not meet the assumption of equality of variances, and the test that does not require this assumption is reported.

Do perceptions of alibi moral desirability affect legal judgments through liking of the

suspect? It is also possible that perceptions of alibi activities and the individuals who engage in them are related to legal judgments via liking of the suspect. Analyses in the previous section 
indicated that Alibi Moral Desirability affected how much participants liked the suspect. As in Experiment 1, mediation analyses using Preacher and Hayes' (2008) method using bias-corrected bootstrapping with 10,000 replacements were conducted to determine whether mediation effects were present. The predictor was the average of participants' perceptions of the morality of the behaviour described in the alibi and an individual who performed this behaviour, the outcomes were participants' legal judgments, and the mediator was participants' liking of the suspect (see Table 35). Participants in the Control condition were excluded from the analyses because they did not rate the morality of the alibi behaviour and actor.

Table 35

Results of mediation analyses

\begin{tabular}{|c|c|c|c|c|c|c|c|}
\hline \multirow[b]{2}{*}{ Outcome } & \multicolumn{2}{|c|}{$\begin{array}{c}\text { Total Effect } \\
(c)\end{array}$} & \multicolumn{2}{|c|}{$\begin{array}{c}\text { Direct Effect } \\
\left(c^{\prime}\right)\end{array}$} & \multicolumn{3}{|c|}{$\begin{array}{r}\underline{\text { Indirect Effect }} \\
95 \% \mathrm{CI}\end{array}$} \\
\hline & $\begin{array}{c}\text { Point } \\
\text { Estimate }\end{array}$ & $p$ & $\begin{array}{c}\text { Point } \\
\text { Estimate }\end{array}$ & $p$ & $\begin{array}{c}\text { Point } \\
\text { Estimate }\end{array}$ & CI LL & CI UL \\
\hline Statement honesty & .008 & .858 & -.036 & .414 & .044 & .008 & .090 \\
\hline $\begin{array}{l}\text { Confidence in statement } \\
\text { honesty }\end{array}$ & .051 & .270 & .023 & .636 & .028 & -.004 & .067 \\
\hline Accuracy & .038 & .372 & -.007 & .878 & .045 & -.094 & .080 \\
\hline Confidence in accuracy & .045 & .295 & .029 & .534 & .017 & -.015 & .058 \\
\hline $\begin{array}{c}\text { Strength of evidence } \\
\text { against suspect }\end{array}$ & -.017 & .724 & -.027 & .603 & .010 & -.030 & .054 \\
\hline Likelihood of guilt & -.029 & .500 & -.003 & .950 & -.027 & -.074 & .007 \\
\hline
\end{tabular}

No significant direct or total effects were found in any of the analyses, meaning that participants' perceptions of the alibi behaviour and actor did not directly predict their legal judgments, whether the mediator was accounted for in the model or not. One significant mediation effect was found; for ratings of statement honesty, the confidence intervals for the indirect effect did not include zero. The more positive participants' ratings of the alibi behaviour and actor, the more they liked the suspect, $r=.341, p<.001$, and the more that participants liked 
the suspect, the more honest they found his statement, $r=.196, p=.004$. The other mediation effects were not significant.

Authoritarianism. Another primary aim of the current study was to explore again whether Authoritarianism was associated with participants' legal judgments. Experiment 1 found, generally, that participants who endorsed more authoritarian views provided responses that were less favourable towards the suspect. Interestingly, participants' confidence in their judgments was not related to any ACT scores. In total, 272 participants answered all questions on the ACT and were included. Details of psychometric analyses are provided in Appendix X. Briefly, after the same three items that were removed in Experiment 1 were dropped, alpha levels were all acceptable, inter-item and item-total correlations were good, and a wide range of scores were observed. Mean scores were very similar across the two experiments and the means in Experiment 2 were also slightly below the midpoint.

Simple regressions. A series of simple regressions were computed to predict participants' responses on the dependent variables using their total and subscale scores on the ACT. Statistical results are available in Table 36 and Table 37. In total, seven of the effects were significant, and no clear pattern emerged in terms of which ACT scores or outcomes were implicated. Although the significant effects that were observed all indicated that higher ACT scores were associated with judgments that were less favourable for the suspect, the majority of the models were not significant. Additionally, with the number of analyses that were conducted, it is possible that at least one of the significant effects is a Type 1 error. Overall, it seems that Authoritarianism is not consistently related to legal judgments.

Mediation effects of alibi moral desirability. One of the important research questions was whether participants' evaluation of the moral desirability of alibi activities mediates the 
relationship between Authoritarianism and participants' legal judgments. It was hypothesized that participants who endorsed more authoritarian views would be more likely to view the morally undesirable alibi negatively and reject the alibi, whereas participants who did not embrace authoritarian views would be more likely to interpret the admission of a morally Table 36

Statistical results for linear regression models using ACT total and subscale scores to predict participants' responses on dependent measures

\begin{tabular}{|c|c|c|c|c|c|c|}
\hline Outcome & Predictor & $b$ & $S E(b)$ & $\beta$ & $R^{2}$ & $p$ \\
\hline \multicolumn{7}{|c|}{ Statement honesty } \\
\hline & ACT & -.009 & .006 & -.095 & .009 & .117 \\
\hline & Conservatism & -.007 & 015 & -.027 & .001 & .654 \\
\hline & Traditionalism & -.023 & 011 & -.118 & .014 & .048 \\
\hline & Authoritarianism & -.019 & .017 & -.065 & .004 & .280 \\
\hline \multicolumn{7}{|c|}{$\begin{array}{l}\text { Confidence in statement } \\
\text { honesty }\end{array}$} \\
\hline & $\mathrm{ACT}$ & .005 & .007 & .044 & .002 & .467 \\
\hline & Conservatism & .017 & .016 & .061 & .004 & .307 \\
\hline & Traditionalism & .002 & .012 & .007 & $<.001$ & .901 \\
\hline & Authoritarianism & .009 & .019 & .029 & .001 & .636 \\
\hline \multicolumn{7}{|c|}{ Alibi accuracy } \\
\hline & ACT & -.002 & .006 & -.024 & .001 & .733 \\
\hline & Conservatism & .008 & .016 & .035 & .001 & .605 \\
\hline & Traditionalism & -.013 & .012 & -.074 & .005 & .280 \\
\hline & Authoritarianism & -.001 & .018 & -.005 & $<.001$ & .947 \\
\hline \multicolumn{7}{|c|}{ Confidence in alibi accuracy } \\
\hline & ACT & .010 & .007 & 106 & .011 & .126 \\
\hline & Conservatism & .027 & .017 & .111 & .012 & .105 \\
\hline & Traditionalism & $<.001$ & .013 & $<.001$ & $<.001$ & .996 \\
\hline & Authoritarianism & .044 & .019 & .156 & .024 & .022 \\
\hline \multicolumn{7}{|c|}{$\begin{array}{l}\text { Strength of evidence against } \\
\text { suspect }\end{array}$} \\
\hline & ACT & .021 & .006 & 194 & .038 & .001 \\
\hline & Conservatism & .062 & .016 & .225 & .051 & $<.001$ \\
\hline & Traditionalism & .022 & .012 & .105 & .011 & .078 \\
\hline & Authoritarianism & .046 & .019 & .148 & .022 & .014 \\
\hline \multicolumn{7}{|c|}{ Likelihood of Guilt } \\
\hline & ACT & .010 & .006 & .103 & .011 & .091 \\
\hline & Conservatism & .019 & .015 & .077 & .006 & .202 \\
\hline & Traditionalism & .014 & .011 & .072 & .005 & .231 \\
\hline & Authoritarianism & .027 & .017 & .095 & .009 & .116 \\
\hline
\end{tabular}


Table 37

Statistical results for binary logistic regression models using ACT total and subscale scores to predict participants' verdict choice and petition signing decision

\begin{tabular}{llcccccc}
\hline Outcome & Predictor & & & & & \multicolumn{2}{c}{$\begin{array}{l}\text { Odds } \\
\text { Ratio }\end{array}$} \\
\hline Verdict Choice & & $b$ & $S E(b)$ & Wald & $R^{2}$ & $p$ & \\
& ACT & -.021 & .009 & 4.96 & .025 & .026 & .980 \\
& Conservatism & -.021 & .023 & .85 & .004 & .358 & .979 \\
& Traditionalism & .029 & .017 & 2.96 & .015 & .085 & .971 \\
& Authoritarianism & -.064 & .027 & 5.82 & .030 & .016 & .938 \\
& & & & & & & \\
Petition Signing & ACT & .008 & .010 & .67 & .004 & .412 & 1.008 \\
& Conservatism & -.025 & .025 & 1.04 & .006 & .307 & .975 \\
& Traditionalism & .048 & .020 & 5.99 & .036 & .014 & 1.049 \\
& Authoritarianism & -.247 & .026 & .019 & $<.001$ & .891 & 1.004 \\
\hline
\end{tabular}

Note. The Nagelkerke $R^{2}$ is reported. Also, note that verdict choice is coded as $1=$ Guilty, $2=$ Innocent. Petition Signing is coded as $1=$ Signed, $2=$ Not Signed.

undesirable act in an alibi to be a sign of honesty and accept the statement. To investigate this question, a series of mediation analyses were conducted using Preacher and Hayes' (2008) biascorrected bootstrapping method with 10,000 replacements. In all analyses, ACT scores were the predictor, participants' legal judgments were the outcome, and participants' evaluations of morality (average of ratings of suspect's alibi behaviour and an adult male who had engaged in the behaviour) were the mediator. Results of some of the models indicated that the bootstrap confidence interval endpoints were not trustworthy, so the number of replacements was increased to 20,000 , and then 50,000, which is the maximum. Despite these increases, the confidence interval endpoints were not trustworthy, even when the interval was set at the minimum of 90 . As a result, all analyses were computed using $95 \%$ confidence intervals with 10,000 bootstrap replacements. Where confidence interval endpoints were not trustworthy, it is noted in the table. As these analyses cannot be easily computed for categorical outcome variables, only results from continuous outcome variables are reported. Missing data were 
deleted case-wise. Statistical results are available in Table 38.

Four of the five significant simple regression effects that were observed were also found in the mediation analyses. However, no significant mediation effects were found for any of the ACT scores on any of the outcome variables. These results show very clearly that participants' perceptions of the activities that the suspect reported engaging in as part of his alibi and their judgments about people who engage in these behaviours did not mediate the relationship between Authoritarianism and legal judgments.

Qualitative analysis of participants' definitions of an "alibi". As in Experiment 1, in addition to providing quantitative ratings of various aspects of the case and the suspect, participants were asked to define the term "alibi." Experiment 1 and Cowan (2012) showed that participants did not necessarily know what the term meant in a legal context, and often reported that a suspect's alibi is another individual who can confirm that a suspect was at another location when a crime was being committed. The majority of participants wrote one to three lines of text, though some participants wrote up to six or seven lines of text. Thirty participants did not provide a response to this item.

The final codes from Experiment 1 were used to code the current data (see Appendix L). No new themes emerged in these data. The data were coded by the same two coders who worked on Experiment 1. In the initial pass, the two coders had complete agreement for $73.0 \%$ and partial agreement for $6.7 \%$ of participants' responses. After the second coder reviewed and revised her codes, identical codes were assigned for $94.4 \%$ of the participants' responses. Partial agreement was found for two participants' responses $(0.8 \%)$. The primary coder's codes were used in the following analysis. 
Table 38

Results of mediation analyses

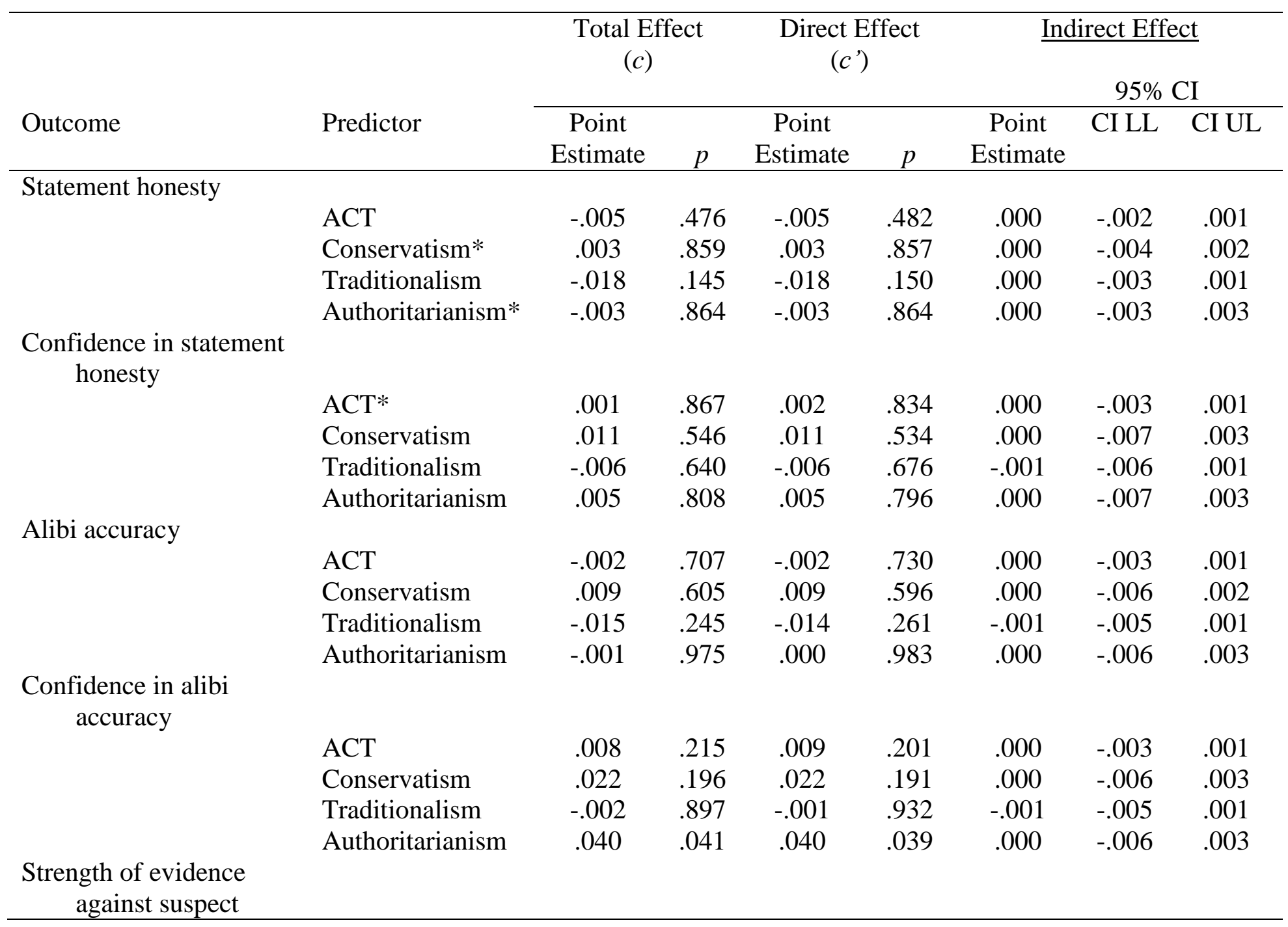




\begin{tabular}{lllllllll}
\hline & ACT & .018 & .019 & .017 & .020 & .000 & -.001 & .002 \\
& Conservatism & .059 & .002 & .059 & .002 & .000 & -.003 & .005 \\
& Traditionalism & .014 & .333 & .014 & .345 & .000 & -.001 & .005 \\
& Authoritarianism & .046 & .037 & .046 & .038 & .000 & -.002 & .005 \\
& & & & & & & & \\
Likelihood of guilt & ACT & .008 & .225 & .008 & .236 & .000 & -.001 & .003 \\
& Conservatism & .018 & .296 & .018 & .301 & .000 & -.002 & .005 \\
& Traditionalism & .008 & .553 & .007 & .576 & .000 & -.001 & .005 \\
& Authoritarianism & .030 & .119 & .030 & .122 & .000 & -.002 & .005 \\
\hline
\end{tabular}


As in Experiment 1, approximately a third (33.6\%) of participants indicated that the alibi is the evidence that supports a narrative, rather than being the narrative itself (code 5 in codebook). In nearly all instances, this code was the only one assigned to the statement.

Another third of participants $(31.6 \%)$ correctly reported that an alibi is a statement that a suspect provides to police about his/her whereabouts during a crime in order to establish that s/he could not be the perpetrator (code 1). This code frequently appeared with other codes. When this code appeared, participants sometimes $(29.6 \%)$ also write that it is generally necessary to provide evidence to support an alibi. Other codes sometimes emerged, but these were infrequent. Interestingly, relatively few participants $(7.4 \%)$ wrote that an alibi is a narrative, but failed to acknowledge that an alibi, in this sense, emerges in the context of a criminal investigation or trial (code 2).

It was also not uncommon for participants' $(13.3 \%)$ response to indicate that $\mathrm{s} /$ he was not familiar with the term "alibi" or be so vague or poorly written that it was not possible to extract any meaning from the statement with any degree of confidence (code 9). This suggests that a notable number of participants do not know what an alibi is.

Finally, a subset of participants (8.2\%) reported that alibis are a cover-up for bad behaviour (code 8). This code was not assigned when participants indicated that an alibi might be deceptive; it was assigned when participants wrote that an alibi was always deceptive or an excuse.

Inferential tests were computed to determine whether participants' different understandings about the term "alibi" (i.e., a narrative vs evidence) affected their judgments about the legal aspects of the case. No significant differences were observed (see Appendix Y). 


\section{Discussion}

Similar to the previous study, Experiment 2 investigated whether having an alibi led to more favourable responses for the suspect relative to not having an alibi and whether the moral desirability of activities described in an alibi affected evaluators' judgments about aspects of a criminal case. Further, the experiment was designed to determine whether the race of the suspect had unique effects and/or interactive effects with alibi content on participants' judgments. The experiment also aimed to replicate Cowan's (2012) results on the influence of Authoritarianism on legal judgments and determine whether participants' impressions of the morality of the alibi mediated these relationships. Finally, there was an attempt to replicate the finding that participants are unfamiliar with the term "alibi."

Failing to generate an alibi may be preferable to providing one. The use of alibis in the legal system is predicated on the assumption that providing an alibi is helpful to innocent criminal suspects. It is entirely logical that a suspect who can provide a narrative for his or her whereabouts at the time of a crime would be judged as more forthcoming and less likely to be guilty than a suspect who does not do so. Laypersons expect that motivation at the time of recall to facilitate memory (Kassam, Gilbert, Swencionis, \& Wilson, 2009), and an innocent suspect in an interrogation is likely to be highly motivated to generate an alibi. Contrary to expectations, when differences were observed, responses that were more favourable for the suspect were provided when the suspect failed to generate an alibi than when he did provide one. None of the analyses showed any benefit to the suspect of providing an alibi. Taken at face value, it appears that providing an alibi may actually be detrimental for suspects. However, not all of the specific alibi narratives showed this pattern, as will be discussed in the following section.

At first glance, this finding seems highly counterintuitive. One possible explanation is 
that an alibi would seem suspicious if it is perceived as the suspect's attempt to cover up his activities during the crime, either because he was involved in the crime or was engaged in other undesirable behaviour. This is consistent with the qualitative responses of a number of the participants, who indicated that an "alibi" involves giving a false statement to police to hide one's involvement in nefarious activities. However, the significant and trending effects were for the strength of the evidence against the suspect, the likelihood of suspect guilt, and the rate of guilty verdicts. Participants rated the suspect's statement as equally honest - and they were equally confident in that decision - regardless of whether he presented an alibi. Mean ratings for statement honesty were near the midpoint of the scale for the combined alibi groups and the control group, suggesting that either participants were uncertain whether the suspect was being honest, or they believed that he was being honest about some aspects of his statement and dishonest about others. Regardless, it seems that the presentation of the alibi did not affect their judgments related to the alibi itself, but had an effect on other judgments, particularly their ultimate judgments in the case.

Alternately, the lack of information provided to participants in Experiment 2 regarding corroboration of the alibi may have implied to participants that the investigators had deemed the suspect's alibi as being so weak that it did not merit investigation. Sommers and Douglass (2007) found that an alibi presented in an investigative context was rated as stronger and as more credible than when the same alibi was presented in a trial context. They suggested that, in the trial context participants assumed that the alibi had already been subjected to scrutiny during an investigation and must have been judged by the investigators as being insufficiently credible. Otherwise, the case would not have proceeded to the trial stage. A similar process may have occurred here. When participants did not receive the follow-up information about the alibi that 
they expected - which was provided in Experiment 1-they may have created a narrative for themselves in which the investigators had determined, for whatever reason, that the suspect was lying or that the alibi was so weak that it was not worth the time and resources required to investigate it. Future studies could investigate this possibility by providing some participants with a document detailing the steps that the investigators will take to investigate the alibi, but without including any information about the results of these steps, and omitting this document for other participants.

\section{The effect of moral desirability on legal judgments and its interaction with suspect}

race. One of the primary aims of this work was to investigate whether the moral desirability of the activities described in an alibi affects evaluators' legal judgments, and whether any effects of moral desirability are consistent for White and First Nations suspects. Results suggested that alibi moral desirability affected some legal judgments, and that these effects varied as a function of suspect race, as expected. Main effects showed that evidence against the suspect was rated as stronger and there were a greater number of guilty verdicts in the Morally Desirable and Neutral conditions than in the Control condition. Interestingly, a number of interaction effects were found such that Alibi Moral Desirability affected ratings for the First Nations suspect, but not for the White suspect. In particular, offering a Morally Undesirable alibi was the most beneficial (or least harmful) scenario for the Indigenous suspect. These effects are consistent with research by Sargent and Bradfield (2004) showing that under low motivation, participants processed information about the strength of the alibi when the defendant was Black and not when he was White. It is possible that participants in the present study processed the alibi information more deeply when the suspect was First Nations than when he was White. An interesting question for future research is whether these apparent differences in information processing have implications 
for memory; it is reasonable to hypothesize that recall might be better in the First Nations condition than in the White condition. Finally, it must be noted, however, that a number of dependent measures showed no significant main or interactive effects for Alibi Moral Desirability.

Interestingly, there was little evidence of suspect race alone affecting participants' attitude ratings. This is surprising, given the general findings in the literature of race effects in the legal sphere, and Marion et al.'s (2011) and Cowan et al.'s (2015) results showing consistent differences in alibi judgments based on whether the suspect was White or Black. In fact, the materials used by Marion et al. (2011) and Cowan et al. (2015) are very similar to those used in Experiment 2, including the suspect information sheet in which suspect race was manipulated. As well, analysis of actual criminal cases supports the assertion that suspects and defendants who are not White are at a systematic disadvantage within the legal system. However, suspect race did affect participants' behaviour; participants were significantly more likely to sign the petition when the suspect was First Nations than when he was White.

One potential explanation for the lack of strong main effects for suspect race is that recent events in Toronto, where the sample was collected, and in Canada more broadly, have made the plight of Indigenous people more obvious to the general population. For example, there have been numerous 'Idle No More' protests in which Indigenous Canadians have been occupying space to push for Indigenous sovereignty and to prevent large corporations from damaging the ecosystem, as well as the push for a public inquiry on Missing and Murdered Indigenous Women (for more details, see www.idlenomore.ca and www.cbc.ca/missingandmurdered/). It is possible that the participants in this study, who were generally young adults from many different racial, ethnic, and cultural backgrounds, living in a 
multicultural environment would be particularly affected by this information. Sommers and Ellsworth's (2001) review showed that African American defendants are treated more harshly than White defendants in the United States only when race is not salient. Participants' awareness of the broader social context in which First Nations people exist in Canada may have led the race information to be more salient than it otherwise would have been, and that this salience obscured a genuine effect. It is reasonable to speculate that the strong, independent effect of suspect race on participants' petition signing behaviour was due to participants' belief that the petition was not part of the study — as overly suspicious participants were removed from these analyses_and likely assumption that there was no need to exert effort to avoid the appearance of racism, though this remains an empirical question.

It would be interesting to compare judgments of Black and White suspects again, now that the general public is aware of the Black Lives Matter (BLM) movement. BLM began in the United States in response to the shooting of Trayvon Martin, an unarmed black teenager in Florida, and the subsequent acquittal of his killer. The movement has expanded and seeks to bring greater awareness of the experiences - particularly with respect to police brutality - of African Americans in North America. On a local level in Toronto, there has been much discussion around police "carding" (i.e., police procedure to stop and document individuals who are not suspected of involvement in any crime, and that tends to disproportionately target People of Colour). Of course, it is also possible that extant research comparing Black and White suspects/defendants cannot reasonably be applied to judgments about First Nations suspects, and that there, in fact, is no systematic difference between judgments made about White and First Nations suspects. 
Crime location and in-group bias. In-group bias was offered as a potential explanation for the differences in the results of Experiment 1 for participants from Iowa State and Ryerson. Consequently, the location of the crime was manipulated in Experiment 2; the crime either took place in Vancouver or Seattle. Participants, all of whom were residents of Canada, were expected to be more likely to identify the suspect from Vancouver as an in-group member than the suspect from Seattle. There were no clear, consistent effects for this variable on questionnaire responses. However, participants' behaviour was significantly affected by crime location. Participants were much more likely to sign the petition supporting the suspect when the crime was set in Vancouver than when it was set in Seattle, which is consistent with what would be expected with an in-group bias.

This result may represent a failure to find a genuine in-group bias effect because the manipulation was not powerful enough to affect participants' ratings. The references to Vancouver and Seattle were relatively subtle in the materials, largely because this manipulation was added after data collection had begun. The location manipulation was more obvious in the petition because the suspect was petitioning either Legal Aid Washington or Legal Aid British Columbia, and this was written in relatively large text on the petition page. It is possible that ingroup bias only showed clear, unique effects on petition-signing behaviour because the information had finally been made salient in the materials, though it could also be because ingroup bias does not affect attitudes and behaviour in the same way. In general, research has shown a poor relationship between attitudes and behaviour (e.g., Batson, Kobrynowicz, Dinnerstein, Kampf, \& Wilson, 1997; Wicker, 1969).

Participant race/ethnicity is unrelated to legal judgments. Unlike in Experiment 1, results of Experiment 2 showed no compelling evidence that participant race/ethnicity was 
related to legal judgments. It should be noted that all participants in Experiment 2 were students at Ryerson, so the null results are, in fact, consistent with the results of Experiment 1. Experiment 1 found differences between White participants at Iowa State and People of Colour (POC) at Ryerson, but did not find any differences between White and POC participants at Ryerson. However, in Experiment 2, a significant difference in the two groups' petition-signing behaviour was observed. White participants were significantly more likely to sign the petition than were POC. This is an interesting finding, and may represent differences in White and POC participants' beliefs in their own ability to affect change. Lower efficacy beliefs for minority than majority individuals have been observed in a number of domains, including healthy eating (Franko, Cousineau, Rodgers, Roehrig, \& Hoffman, 2013), performance in STEM programs (MacPhee, Farro, \& Canetto, 2013), and avoiding drug use (Hives, 2016). This belief in one's

own efficacy is an essential component in eliciting goal-directed behaviour (e.g., Reeve, 2005). It is conceivable, then, that higher levels of self-efficacy led White participants to be more likely to take action in the form of signing the petition.

\section{Participants' general feelings about First Nations and White people are unrelated to}

their legal judgments. It was hypothesized that participants' general attitudes towards First Nations and White people might influence their responses to First Nations and White suspects such that more negative attitudes would be associated with ratings that are less favourable to the suspect. However, the data did not show this pattern; for nearly all analyses, participants' general feelings about First Nations and White people were unrelated to their judgments in the case. However, more positive feelings about White people were associated with a higher rate of signing the petition for the White suspect. As noted earlier, participants may have been less likely to moderate their behaviour to avoid appearing racist with the petition than for other 
measures, as the petition was presented as being unrelated to the study.

The lack of significant effects was likely at least partly due to the high proportion of participants who provided highly positive ratings. However, these high ratings may not represent participants' true feelings about First Nations and White people. Participants may have intuited the purpose of the items, or may have been motivated to appear generally tolerant of others. Alternately, the data were collected in what is arguably the most multicultural city in Canada; half of Toronto's citizens were born outside of Canada and 47\% of Toronto residents identify themselves as "persons, other than Aboriginal peoples, who are non-Caucasian in race or nonwhite in colour" (City of Toronto, n.d.). It is possible that the extensive exposure to individuals from different countries, cultures, and religions led our participants to have particularly favourable views towards individuals from many groups. The finding that a subset of participants did provide fairly negative ratings for First Nations and/or White people suggests that at least some of the participants were not concerned with keeping up appearances. However, the current data do not allow a firm conclusion as to whether participants were entirely honest in their ratings. Future studies could attempt to capture participants' attitudes about race using more subtle means or a questionnaire that includes methods to identify when participants are engaging in impression management. One example would be assessing participants' feelings about the relevant outgroup by setting up two chairs in the testing room or waiting area that are uncomfortably close, leading the participant to expect s/he will be sitting next to a member of that group, and measuring the distance between the chairs after the participant is given an opportunity to move one of them. Alternately, participants could be required to complete a race implicit association test (IAT) in prescreen measures before signing up for the study. 


\section{Judgments of similarity, liking, and the overall evaluation of the suspect's character.}

In general, participants thought that they were not particularly similar to the suspect, they did not particularly like him, and thought he was a somewhat bad person overall. This pattern may be due to the context in which they encountered the target individual; anyone may be tainted by suspicion when $\mathrm{s} / \mathrm{he}$ is a suspect in a murder case. There may be a widespread presumption that an individual must have done something to become a suspect in a criminal case.

Alibi Condition had a significant effect on these judgments. The general pattern across all three dependent variables was that more negative ratings were provided in the Morally Undesirable condition compared to the other conditions. If participants already believed that a criminal suspect must have done something negative to draw the attention of law enforcement, the revelation of further negative behaviour would be consistent with these already-held beliefs and could polarize judgments. In contrast, participants may have rejected or downplayed information that was positive or neutral because it did not fit with their existing schemas of criminal suspects or criminal investigations. Essentially, the pattern of results could represent confirmation bias by participants, as they attempt to validate their negative beliefs about criminal suspects. Finally, participants' liking of the suspect did not mediate the relationship between perceptions of the morality of the alibi and legal judgments.

Authoritarianism and the mediational effects of perceived alibi moral desirability. Overall, no clear, consistent pattern of associations were found between scores on the ACT and its subscales and participants' legal judgments. Where results were significant, higher ACT scores were related to ratings that were less favourable for the suspect. However, there was no identifiable pattern with respect to which predictor or outcome variables showed significant effects. Without a clear, interpretable pattern, it is impossible to say what effect, if any, 
participants' views about authoritarian ideals influence their judgments in the legal context.

As well, participants' impressions of the moral desirability of the alibi did not significantly mediate any relationship between ACT scores and legal judgments. This is not consistent with the hypothesis that higher authoritarian participants would be more likely to react negatively to the morally undesirable alibi and choose to reject it and punish the suspect who offered it, compared to individuals who did not endorse authoritarian views.

Theoretical considerations. At the outset of this program of research, the intention was to compare two competing theories that both suggested that alibi moral desirability would affect legal judgments. In the first, which was supported by general principles of social psychology (e.g., halo/horns effect, what is beautiful is good stereotype), participants were expected to create an implicit personality theory about the suspect that was consistent with the nature of his alibi activities. A morally undesirable alibi would lead to a negative implicit personality theory and a morally desirable one should lead to a positive implicit personality theory. A negative implicit personality theory would manifest in lower ratings of statement honesty, higher likelihood of guilt ratings, et cetera, than the positive implicit personality theory. In the second theoretical rationale, participants would interpret the admission of morally undesirable behaviour in the alibi as indicating honesty and be more likely to believe the alibi and less likely to think that the suspect was guilty, relative to other alibis.

The general pattern of main effects for alibi content was that less favourable ratings were provided for the Morally Desirable and sometimes for the Neutral condition than either the Control condition or the Morally Undesirable condition. With ratings of suspect honesty and alibi accuracy, this pattern was found for First Nations suspects only. These results are the opposite of what would have been predicted by the first theory, as the Morally Desirable alibi 
was not beneficial for suspects, nor did the Morally Undesirable alibi have detrimental effects. It is not entirely consistent with the second theory, either. Although the Morally Undesirable alibi was the most highly rated of the alibis, it was not significantly better than the No-Alibi Control. In the theory, as presented, offering a morally undesirable alibi was expected to help suspects. Instead, the results showed that offering a morally undesirable alibi merely did not hurt the suspect, unlike the other alibis. Indeed, the conclusion from these results would be that offering an alibi that describes prosocial activities may be perceived by evaluators as self-serving. Research on deception has shown that individuals lie to maintain someone else's positive impression of them or to avoid damaging this positive impression (e.g., Aune \& Waters, 1994). It is not inconceivable, then, that a suspect who is generating a false alibi would be most likely to choose a narrative that casts him or herself in a positive light.

Participants' understanding of the term "alibi." The results of the qualitative coding suggest that many participants misunderstood the term "alibi." Just over one third of participants indicated that an alibi was the evidence - usually in the form of testimony from another individual - that supported a narrative, rather than being the narrative itself. This was the most common response across the whole sample. As well, $13.3 \%$ of participants did not know the term; they either wrote that they were unfamiliar with the word, or their response did not communicate in any coherent way that they understood the term. A small number of participants asked the experimenter what the word "alibi" meant. Only approximately a third of participants provided responses that indicated that they knew that an alibi refers to a narrative placing a criminal suspect somewhere other than the scene of a crime at the relevant time. The high degree of misunderstanding is consistent with the results of Experiment 1, and with previous research by Cowan (2012). As in Experiment 1, participants' legal judgments were unaffected by whether 
participants understood that an alibi refers to a narrative regarding a suspect's whereabouts at the time of a crime or as evidence supporting a narrative.

Limitations. The chief limitation of the current study was that the Morally Desirable alibi may have been viewed as relatively neutral. Manipulation check items indicated that participants rated the Morally Desirable behaviour as somewhat positive, but this did not spill over onto their judgments about an individual who had engaged in this action. As well, no significant differences were observed between the Morally Desirable and Neutral groups on their ratings of how similar they thought they were to the suspect, how much they liked the suspect, and their overall evaluation of the suspect's character, though, as noted earlier, these ratings may have been influenced by the fact that the information was presented in the context of a murder investigation. It is also possible that these results represent a negativity bias - the tendency for individuals to provide greater weight to negative information than to positive information (e.g., Rozin \& Royzman, 2001; Skowronski \& Carlston, 1987). With that in mind, it is perhaps not surprising that large, significant differences were found between the Morally Undesirable and Neutral alibis and that moderate or non-significant differences were found between the Morally Desirable and Neutral alibis. Finally, it is possible that the text of the Morally Desirable alibi itself failed to paint as positive an impression as had been intended. In this alibi, the suspect was ostensibly helping his friend's romantic partner move residences. This behaviour may have been seen as a typical action that friends are expected to do for each other as part of the social contract. Additionally, the scenario could have evoked a number of different narratives, from very hard work lifting heavy boxes or furniture and cleaning to sitting around and packing a few boxes while socializing. The former was what was intended, but participants may have envisioned a situation more similar to the latter. Indeed, when participants were asked to report 
the alibi in an open-ended item, a few indicated that the suspect was helping his friend's partner pack or unpack, implying that there was no heavy lifting involved. None of the participants implied in those responses that they believed that the suspect was at the house, but was engaged in any non-moving-related activities (e.g., having an affair).

It is also possible that a difference between alibi conditions other than moral desirability is responsible for the observed effects. To ensure that there were a minimum of differences between the alibi conditions, the alibi always took place at the same location-the friend's home - and with the same possibility for corroboration. The only difference between these conditions was the activity itself. It is possible, however, that the key difference in the activity was not moral desirability. The results from Experiments 1 and 2 showed that many participants had helped a friend move or had picked up items they had left at a friend's house, but relatively few had had an affair with a friend's partner. However, this latter alibi appears to have operated as intended; the behaviour was rated as negative in both of the current studies, the earlier pilot test, and in Cowan (2012). Still, future studies would be best served by attempting to select alibis with which participants would have equal experience.

On the other hand, the results of the analyses examining the effect of Alibi Moral Desirability and the other independent variables did show some differences between the Morally Desirable and Neutral conditions, in particular that some effects were significant for Morally Desirable, but trending for Neutral. This suggests that participants did react differently to the two narratives. It is unclear what factor other than moral desirability was responsible, as the other elements of the narrative (e.g., setting, presence of others, time) were consistent between the two conditions. Still, future studies should attempt to provide participants with an unequivocally morally desirable alibi for that condition. In fact, it may be preferable to provide a Morally 
Desirable alibi that is even more strongly positive than the Morally Undesirable alibi is negative to overcome any influence of a general negativity bias.

It is also possible that the manipulation check items themselves were ineffective at identifying differences. Participants rated their impressions of the alibi activity and an adult who engaged in that activity on a scale ranging from "Very Negative" to "Very Positive." The item was written using this wording to ensure that the item was as clear, simple, and understandable as possible. However, it may have been preferable to ask participants directly for their assessments of how morally good or bad the behaviour and actor were. For example, it is highly likely that participants would assess a serious moral transgression, and therefore the actor, as both being very negative. Although the two concepts overlap, it is not clear that they are entirely analogous.

A second limitation was related to the petition. There were many improvements to the petition between Experiment 1 and Experiment 2 based on participant feedback and an examination of genuine petition websites. However, a notable subset of participants (7.3\%) still indicated that they were suspicious enough about the petition to justify removing their data from analysis. This figure is comparable to the percentage of highly suspicious participants in Experiment 1 (6.0\%). Additionally, other participants reported considering the possibility that the petition was part of the study. It is not clear whether or how these lower levels of suspicion may have affected participants' behaviour. It is also possible that, with group testing, when one participant disclosed suspicion about the petition, the other participants may have become more likely to report suspicion because they did not wish to admit that they had been duped. This type of procedure may be less likely to be effective with introductory psychology students, who are learning in class about social psychology studies that have used deception or incomplete 
disclosure, relative to naïve participants.

Despite the challenges in measuring behaviour, it is a valuable endeavour, given that the end goal of research in social psychology and in psychology and law generally involves making claims about behaviour. One contribution of the current work is including a behavioural measure. Solving this problem of participant suspicion could take one of two forms: changing the procedure around presenting the petition or measuring another form of behaviour. Suspicion would likely be lessened if there was a time gap between the apparent end of the experimental session and the presentation of the petition, and if the petition was presented by an individual with no obvious involvement in the research, for example a confederate near the exit of the building. Rather than being directly related to the case, the petition would have to relate to a more general topic about justice, or, perhaps a genuine potential wrongful conviction case. Naturally, participants would then have to be re-debriefed and asked for consent due to the deception. There would also be substantial risk of participants in later sessions witnessing the petition procedure when they are arriving for their appointments, so the timing of the sessions would have to be spaced in such a way to reduce the possibility of this occurring. In addition, it is possible that some participants would be missed if the confederate was busy presenting the petition to others, or if participants scheduled another experiment session and stayed in the waiting room. Overall, there are considerable challenges with using a petition as a measure of behaviour.

It would also be possible to measure an entirely different form of behaviour. For example, participants could be led to believe that the suspect in the case was found not guilty at trial, and that they might have the opportunity to meet him. Participants who believe that the suspect was guilty, or that he was innocent but is a bad person, would presumably be less willing 
to be in the same physical space as the suspect than participants who do not have negative views about him. A behavioural measure, then, could be whether participants include their name on a raffle list for tickets to an event where the suspect is a speaker. However, this is arguably unethical, as participants could be highly disappointed to learn that the event will not actually occur. Another option would be to pay participants for their participation and then ask them what proportion of their remuneration, from $0-100 \%$, they would like to donate to the suspect's defense fund, or to a wrongful conviction charity (for examples, see Grusec, Kuczynski, Rushton, \& Simutis, 1978; Grusec \& Redler, 1980; Miller, Kahle, \& Hastings, 2015; Sharma, 1988). Naturally, it would be preferable for the literature to include a range of behavioural measures to ensure that any significant effects generalize.

The photos shown to participants on the suspect information sheet may be a third limitation to the current study. Despite attempts in pilot testing to choose photographs that were similar on a number of relevant dimensions, participants rated one of the First Nations suspects as being a slightly worse person than the other First Nations suspect. No differences were observed between participants' ratings of how much they liked the two First Nations suspects, and no differences on either measure were found between the two White suspects. As a result of the one discrepancy, a variable was included in all relevant analyses that represented which photo was used. No interactions between that variable and Suspect Race were observed, suggesting no difference in participants' responses to the dependent measures between the two photos within each Suspect Race category. It seems that the initial difference observed between the two First Nations suspects did not affect the results, suggesting that the results can be generalized beyond the specific photos that were chosen to First Nations and White suspects more generally. 
Conclusions. The results of Experiment 2, in isolation, lead to eight general conclusions:

1. Presenting an alibi may be detrimental for suspects. This effect may occur when evaluators interpret an alibi as referring to a cover-up for bad behaviour, or when aspects of the context imply that investigators have determined that the alibi is so weak, or so obviously fabricated that it need not be investigated further.

2. The content of a suspect's alibi may affect participants' judgments. For legal judgments, it seems that offering an alibi that describes prosocial activities is seen as suspicious. However, presenting an alibi that includes negative behaviour is not helpful; participants' responses to the Morally Undesirable alibi were not significantly different than their responses in the Control condition, where the suspect did not offer an alibi.

3. Alibi content affects non-legal judgments. Although participants reported not particularly liking the suspect, thinking that the suspect is relatively dissimilar from them, and thinking that he was a somewhat bad person, this was particularly the case when he offered a Morally Undesirable alibi. Ratings were the least unfavourable with a Morally Desirable alibi. However, it is not clear whether these broad impressions about the suspect himself have broader effects; the degree to which participants liked the suspect did not mediate the relationship between perceptions of the moral desirability of the alibi and their legal judgments.

4. No clear evidence was found that suspect race has a strong, direct, unique effect on legal judgments, though in some cases it affected whether participants' responses were influenced by alibi content. In particular, alibi content effects were found when the suspect was First Nations and not when the suspect was White. Additionally, there was no evidence that participants' general feelings about First Nations and White people 
affected their legal judgments, though the vast majority of ratings were highly positive.

5. Participants' own race/ethnicity was unrelated to legal judgments.

6. Strong evidence for in-group bias was not found by manipulating whether the suspect was Canadian or American, with an entirely Canadian sample. However, the manipulation in the police file was quite subtle, and the manipulation affected petition signing behaviour, where the manipulation was much more obvious.

7. There was no clear, consistent pattern of effects for Authoritarianism on legal judgments, and there was no evidence for perceptions of the morality of the alibi mediating this relationship.

8. The majority of participants did not provide an accurate definition of the term "alibi." 


\section{Experiment 3}

\section{Introduction}

Results thus far suggested a few general conclusions. First, although alibi moral desirability affected some of participants' judgments, the patterns of effects were not consistent between the various samples and did not provide clear support for either of the proposed hypotheses. In-group bias was proposed as an explanation for the differences between the Ryerson University and Iowa State University samples in Experiment 1, though Experiment 2 did not provide strong evidence for this explanation. Contrary to expectations, suspect race showed no clear effects on responses and participants' general feelings about White and First Nations people were unrelated to legal judgments in Experiment 2. However, the predicted effect that strong evidence to corroborate an alibi would be helpful to suspects was found in Experiment 1. A mix of significant and non-significant effects was found for Authoritarianism across the first two experiments, though all significant effects were in the predicted direction of higher scores being associated with harsher judgments. Finally, observed results substantiated previous literature that many participants do not know what an alibi is. As a result, the primary aims of Experiment 3 were to: (a) investigate the effect of in-group bias on legal judgments, (b) clarify whether evaluators' impressions of a case vary depending on whether the suspect/defendant is First Nations or White, (c) determine whether Authoritarianism is related to legal judgments, (d) consider the possible effects of participants' religious orientation, which will be discussed further below, and (e) replicate the results of the previous studies on participants' understanding of an "alibi."

In the 2011 Canadian National Household Survey, $76.1 \%$ of respondents reported a religious affiliation, the majority of whom identified as Christian (67.3\% of Canadians) 
(Statistics Canada, 2011). The US Census Bureau is barred by law (Public Law 94-521) from asking mandatory questions on religious affiliation, though some of their surveys have collected these data on a voluntary basis. One such survey showed that $84.2 \%$ of respondents who answered the question reported a religious affiliation, and $80.1 \%$ of the respondents were Christian (U.S. Census Bureau, 2012). However, these individuals likely differ in the strength of their religious beliefs. Religion is an important aspect of many people's lives and it has yet to be investigated whether participants' judgments regarding alibis, or legal issues more broadly, are related to evaluators' religious beliefs.

Although initially, religious orientation was described as either being intrinsic or extrinsic (e.g., Allport, 1954, as referenced by Hills, Francis, \& Robbins, 2005), a third motivation - quest - has since been included (Batson, Schoenrade, \& Ventis, 1993, as referenced by Hills et al., 2005). These are different approaches to religion, but are not mutually exclusive. In Extrinsic orientation, religious observance is seen as a means to an end. Here, religious practice is focused on its utility - which may include security and solace, opportunities to socialize, distraction, status, and self-justification - and generally involves religious beliefs that are weakly held and deployed selectively to meet the individual's other, more important needs (Hills et al., 2005). In Intrinsic orientation, religious beliefs are internalized and the needs associated with religious observance are primary; individuals amend their other needs to be consistent with the requirements of their religious practice (Hills et al., 2005). Finally, Quest orientation involves questioning teachings, exploring aspects of religion and faith for oneself, and embracing and valuing doubt as an avenue for spiritual growth; it is a thoughtful, intellectual approach to religion that rejects the notion of dogmatic adherence to religious teachings (Hills et al., 2005).

For the purposes of the present study, it was hypothesized that higher Extrinsic 
orientation would be associated with legal judgments that are less favourable for the defendant. In particular, the need for status and security were anticipated to lead these individuals to perceive themselves as superior to the defendant and worthy of judging him. It is possible that higher Intrinsic orientation would be associated with ratings that are either more or less favourable to the defendant. These individuals hold their religious beliefs as central aspects of themselves. If this manifests in a belief that all others should follow the same behavioural rules as the self, this could lead to less favourable responses for the defendant. However, if individuals take seriously the teaching in many religious traditions to be kind and non-judgmental towards others, this may lead to relatively more favourable ratings for the defendant. It is unclear whether or how Quest orientation may be related to legal judgments, so no formal hypotheses are offered. Canadian and American participants, recruited through Amazon's Mechanical Turk read a version of a fictitious newspaper article describing, in broad strokes, an upcoming trial. The defendant's ethnicity (First Nations or White) as well as the location of the crime (Seattle, WA or Vancouver, BC) were manipulated between subjects. This latter manipulation was used as a proxy for defendant nationality (i.e., Canadian or American). Participants answered questions about their impressions of the case itself and provided a definition of an "alibi." They then completed the Authoritarianism-Conservatism-Traditionalism scale (Duckitt et al., 2010) and the Revised Religious Life Inventory (RLI-R; Hills, Francis, \& Robbins, 2005). Finally, they responded to the same thermometer items to assess their feelings about First Nations and White people in general, as in Experiment 2. The study was approved by the Research Ethics Board at Ryerson University and the procedures and practices employed in the study complied with the ethical guidelines mandated by the most recent Tri-Council Policy Statement. 


\section{Method}

Design. The study was a 2 (Sample: Canadian, American) X 2 (Crime Location: Seattle, Vancouver) X 2 (Defendant Race: First Nations/American Indian, White) design, with all variables manipulated between subjects.

Materials. Participants were presented with a fictitious article describing an impending criminal trial in a second degree murder case (see Appendix CC). The article was designed to mimic the writing style and layout of genuine online newspaper articles. These articles generally include an overview of the crime, details about the victim and the suspect, and information about the investigation, all in very short paragraphs. Broadly, the article presented to participants described a murder that took place during a robbery in an apartment, in which a witness recalled seeing either a First Nations or White man leaving the building on the night of the crime. Fingerprints were matched to the defendant, who acknowledged being in the apartment three days earlier when he had been hired to fix the sink. The article finished by noting that the defendant is anticipated to present an alibi defense at trial, and that his alibi has been confirmed by his friends. Some corroboration was arguably necessary to ensure that the article included evidence that suggested the defendant's innocence as well as evidence for guilt (i.e., fingerprints, witness statement) so as to avoid ceiling or floor effects. This would be considered relatively weak corroboration, as it is provided by motivated familiar others. This was chosen due to results of Experiment 2 that indicated that other variables may only be influential with weak or absent corroboration. The name of the newspaper — either Vancouver or Seattle Tribune-was manipulated to be consistent with where the alleged crime occurred (i.e., Vancouver or Seattle), but neither have a 'Tribune'; this aspect was fabricated because it was anticipated that participants' impressions of genuine news sources might influence their impressions of the case 
materials. The defendant's race/ethnicity was manipulated within the witness' statement, and also by changing the name of the defendant. The White suspect was named Joseph Campbell, a surname that is very common in Scotland, whereas the First Nations suspect was named Joseph Crowfoot, a surname common to Indigenous people.

The first section of the questionnaire assessed demographic information, including age, gender, race/ethnicity, and details about the province or state in which they currently reside (see Appendix DD). They were also asked about their experience with Mechanical Turk, an online data collection tool provided by Amazon. Participants responded to the same case-specific questions as in Experiments 1 and 2, though the word "defendant" was used in place of "suspect." Participants then rated how similar they are to the defendant generally, and in terms of personality, and how different they are in terms of activities they enjoy, on a scale of 1 (Not at all) to 7 (Very). They also rated how much they thought they would like the defendant if they met him on a scale from 1 (Like a great deal) to 7 (Dislike a great deal), the likelihood the participant would become friends with the defendant if they met, on a scale from 1 (Not at all likely) to 7 (Very likely). Next, they rated how they would feel if the defendant lived next door, was a coworker, or was involved romantically with one of the participant's family members, on a scale from 1 (Very negative) to 11 (Very positive). Finally, they rated the defendant's overall character on a scale from 1 (A very bad person) to 11 (A very good person).

The next section of the questionnaire included multiple choice manipulation check items about the defendant's alibi, the race/ethnicity of the man that the witness said she saw leaving the apartment building that night, and the city where the crime took place, as well as easy general knowledge questions (e.g., tallest mountain in the world) to identify if the study was completed by a pre-programmed script, rather than by a human participant. Participants were then asked to 
define the term "alibi" in an open-ended item. As some of the participants in Experiments 1 and 2 only recognized the word "alibi" when it was spoken aloud, a written phonetic approximation of the term was also provided. On a separate page, participants were asked whether the definition they had provided was what they already knew about the word, or whether they had searched the internet to find a definition for the term and provided that. Finally, they were asked to provide general comments about the case in an open-ended format.

After participants completed the case-relevant questionnaire sections, they were given the ACT (Duckitt et al., 2010). The scale included three subscales (i.e., Authoritarianism, Conservatism, and Traditionalism), with six items each, half of which are reverse-scored. On this measure, participants rate their level of agreement with the statements on a scale from 1 (Strongly Disagree) to 7 (Strongly Agree).

Next, participants completed an edited version the RLI-R (Hills, Francis, \& Robbins, 2005; see Appendix EE for original and edited wordings). This measure includes three subscales (Extrinsic [7 items], Intrinsic [9 items], and Quest [8 items]), that all had alpha levels above .75 in Hills et al.'s (2005) report. For all items, participants rate their level of agreement on a scale from 1 (Strongly Agree) to 9 (Strongly Disagree). None of the items are reverse-scored.

Two minor changes were made to the RLI-R published by Hills et al. (2005). First, a "Not Applicable" option was added to all items to identify individuals who do not hold religious beliefs or engage in religious practices. The second minor alteration was to substitute "place of worship" for "church" and "religious text" for "Bible" in all relevant items so that the scale is appropriate to many faiths or spiritual practices.

Finally, participants completed a series of thermometer items on which they rated their general feelings about various groups (e.g., African Americans, senior citizens, bisexual people) 
on a visual analogue scale ranging from 0 (Cold) to 100 (Warm). This set of items was nearly identical to those used in Experiment 2. The key responses were participants' feelings about First Nations/American Indian/Indigenous People and Caucasian/White people. The former was edited to include American Indian, as this is the term that would be most familiar for residents of the United States. First Nations is a more common term in Canada. The other items were included to obscure the purpose of the thermometer items. The presentation order of the various groups was randomized.

Procedure. Participants were recruited online through Amazon's Mechanical Turk (mTurk) website. To ensure equal representation of Canadians and Americans, and equal cell sizes across both nationalities, the study was advertised separately for Canadian and American participants (see Appendix AA). Participants were paid \$1 USD for their participation. As this was an online study, participation occurred wherever and whenever participants chose. The survey itself was hosted on Qualtrics. After participants had consented to participate in the study, they responded to the demographic items, read a version of the newspaper article, and completed the rest of the questionnaire. On average, participants completed the study in 15-20 minutes.

Participants. The study was completed by 152 Canadian and 146 American participants. A priori power analyses using $G *$ Power 3.1.7 (Faul et al., 2007) suggested a sample size of 199 for this design, with an estimated effect size of .20. However, it was expected that some participants would fail the manipulation check items, so additional participants were sought. Sixty-three participants' data were removed because they provided incorrect responses to the manipulation check items about crime location and suspect race or did not respond to these items, resulting in a final sample of 235 .

The sample used in analyses comprised 128 Canadian and 107 American participants. 
Demographic information is provided in Table 39. On average, participants were in their 30s.

The American sample was significantly older than the Canadian sample, $t(192.86)=3.87, p<$ $.001, d=0.52$, but both samples included a fairly even split of men and women, and both were predominantly White.

Table 39

Participant demographic information

\begin{tabular}{lccc}
\hline & Canadian & American & Combined \\
\hline Age & & & \\
Mean & 32.51 & 38.14 & 35.06 \\
Standard Deviation & 9.37 & 12.35 & 11.16 \\
Median & 31.00 & 34.50 & 32.00 \\
Range & $18-60$ & $21-71$ & $18-71$ \\
Gender & & & \\
Women & $54(42.2 \%$ & $52(48.6 \%)$ & $106(45.1 \%)$ \\
Men & $72(56.3 \%)$ & $54(50.5 \%)$ & $126(53.6 \%)$ \\
Trans* & $1(0.8 \%)$ & $0(0 \%)$ & $1(0.4 \%)$ \\
Queer & $3(2.3 \%)$ & $0(0 \%)$ & $3(1.3 \%)$ \\
Other & $1(0.8 \%)$ & $0(0 \%)$ & $1(0.4 \%)$ \\
Racial/Ethnic Group & & & \\
American Indian or Alaska Native & $0(0 \%)$ & $1(0.9 \%)$ & $1(0.4 \%)$ \\
Black or African American & $4(3.2 \%)$ & $10(9.3 \%)$ & $14(6.0 \%)$ \\
East Asian & $5(3.9 \%)$ & $5(4.7 \%)$ & $10(4.3 \%)$ \\
Hispanic or Latino/a & $3(2.3 \%)$ & $2(1.9 \%)$ & $5(2.1 \%)$ \\
Indigenous Canadian (First Nations, & $4(3.1 \%)$ & $2(1.9 \%)$ & $6(2.6 \%)$ \\
$\quad$ Inuit, Métis) & & & \\
South/Southeast Asian & $9(7.0 \%)$ & $1(0.9 \%)$ & $10(4.3 \%)$ \\
West Asian/Middle Eastern & $3(2.3 \%)$ & $0(0 \%)$ & $3(1.3 \%)$ \\
White & $101(78.9 \%)$ & $90(84.1 \%)$ & $191(81.3 \%)$ \\
Other & $1(0.8 \%)$ & $0(0 \%)$ & \\
\hline
\end{tabular}

Note. One American participant did not provide responses to any of the demographic items.

The Canadian sample represented all provinces except Prince Edward Island - but no territories - with the majority from Ontario, Quebec, and British Columbia. The American sample included participants from 29 different American states. The vast majority of the participants (80.5\% Canadian participants, 76.4\% American participants) have lived in their current state or province for 11 years or longer. In general, Canadian participants identified 
strongly as being Canadian $(M=6.13, S D=1.37)$ and were proud of being Canadian or living in Canada $(M=5.84, S D=1.54)$. Similarly, American participants identified strongly as being American $(M=5.92, S D=1.27)$ and were proud of being American or living in the United States $(M=5.50, S D=1.43)$. The maximum possible score on each of these items was 7 . No significant differences were observed between the two samples for identification with country of residence, $t(232)=1.20, p=.233, d=0.16$, or pride in country of residence, $t(232)=1.77, p=$ $.081, d=0.23$.

The majority of the Canadian participants (92.9\%) had been signed up as workers on Mechanical Turk for 6 months or less. For American participants, there was substantial variability in the length of time participants had been signed up with Mechanical Turk; 30.8\% less than 6 months, $16.0 \%$ six months to one year, 23.6\% 1-2 years, 25.5\% 3-5 years, and 3.8\% 6-10 years.

\section{Results}

\section{The effect of defendant race, crime location, and sample location on legal}

judgments. Two primary aims of Experiment 3 were to investigate whether in-group bias (i.e., interaction between Crime Location [Vancouver or Seattle] and Sample [Canadian or American]) and Defendant Race (White or First Nations) affected participants' judgments about the legal aspects of the case. A series of univariate ANOVAs were conducted, with all variables manipulated between participants.

Defendant honesty. Participants rated how honest the defendant was in his description of his whereabouts during the crime. No significant effects were observed (See Table 40), though there was a trend for honesty ratings to be higher when the crime was set in Seattle $(M=4.86$, $S D=1.47)$ than when it was set in Vancouver $(M=4.50, S D=1.33)$. Given these means, it 
seems that participants were generally unsure about the defendant's honesty.

Table 40

ANOVA results for participants' ratings of the defendant's honesty

\begin{tabular}{lccc}
\hline Effect & $F$ & $p$ & $\eta_{\mathrm{p}}^{2}$ \\
\hline Defendant Race & 2.98 & .086 & .013 \\
Crime Location & 3.52 & .062 & .015 \\
Sample Location & .52 & .470 & .002 \\
Defendant Race X Crime Location & .002 & .968 & $<.001$ \\
Defendant Race X Sample Location & 1.13 & .289 & .005 \\
Crime Location X Sample Location & 1.24 & .267 & .005 \\
Defendant Race X Crime Location X Sample Location & .01 & .907 & $<.001$ \\
\hline Note. All
\end{tabular}

Note. All effects are $d f=1,226$

Confidence in defendant honesty. No significant effects were found for participants' confidence in their ratings of defendant honesty (see Table 41). The overall sample mean was $4.28(S D=1.75)$, suggesting that participants were not particularly confident in their decisions.

Table 41

ANOVA results for participants' confidence in their ratings of the defendant's honesty

\begin{tabular}{lccc}
\hline Effect & $F$ & $p$ & $\eta_{\mathrm{p}}^{2}$ \\
\hline Defendant Race & 1.34 & .248 & .006 \\
Crime Location & 3.06 & .082 & .013 \\
Sample Location & .57 & .452 & .002 \\
Defendant Race X Crime Location & 1.23 & .268 & .005 \\
Defendant Race X Sample Location & 2.32 & .129 & .010 \\
Crime Location X Sample Location & .26 & .610 & .001 \\
Defendant Race X Crime Location X Sample Location & .28 & .594 & .001 \\
\hline
\end{tabular}

Note. All effects are $d f=1,227$

Alibi accuracy. No significant effects were found for participants' ratings of the accuracy of the defendant's alibi (see Table 42). Overall, participants indicated that the alibi was moderately accurate $(M=4.90, S D=1.55)$. 
Table 42

ANOVA results for participants' ratings of alibi accuracy

\begin{tabular}{lccc}
\hline Effect & $F$ & $p$ & $\eta_{\mathrm{p}}^{2}$ \\
\hline Defendant Race & 2.16 & .143 & .009 \\
Crime Location & 1.93 & .167 & .008 \\
Sample Location & .45 & .506 & .002 \\
Defendant Race X Crime Location & .61 & .437 & .003 \\
Defendant Race X Sample Location & .87 & .353 & .004 \\
Crime Location X Sample Location & 1.42 & .234 & .006 \\
Defendant Race X Crime Location X Sample Location & .05 & .831 & $<.001$ \\
\hline
\end{tabular}

Note. All effects are $d f=1,226$

Confidence in alibi accuracy. One significant and one marginally significant effect were found for participants' self-reported confidence in their rating of the accuracy of the defendant's alibi (see Table 43). Participants were significantly more confident when the crime was set in Seattle $(M=4.86, S D=1.66)$ than in Vancouver $(M=4.33, S D=1.80)$, regardless of whether they themselves were Canadian or American. Crime Location was also implicated in a marginally significant interaction with Defendant Race (see Figure 9; throughout, Masson and Loftus' [2005] between-subjects error bars are used). Follow-up tests indicated that when the crime was set in Seattle, participants were significantly more confident in their ratings for the White defendant than the First Nations defendant, $t(108)=1.97, p=.051, d=0.38$, but there was no significant effect of Defendant Race when the crime was set in Vancouver, $t(112)=.65, p=$ $.518, d=0.12$. It seems as though the interaction is due primarily to the higher confidence ratings from the group who read about a White defendant in Seattle. This result was unanticipated and there is no theoretical rationale of which we are aware to explain it. Care is necessary in the interpretation, as the interaction was only moderately significant. 
Table 43

ANOVA results for participants' confidence in their ratings of alibi accuracy

\begin{tabular}{lccc}
\hline Effect & $F$ & $p$ & $\eta_{\mathrm{p}}^{2}$ \\
\hline Defendant Race & .97 & .327 & .004 \\
Crime Location & 4.14 & .043 & .018 \\
Sample Location & .24 & .626 & .001 \\
Defendant Race X Crime Location & 3.49 & .063 & .015 \\
Defendant Race X Sample Location & .02 & .895 & $<.001$ \\
Crime Location X Sample Location & .85 & .358 & .004 \\
Defendant Race X Crime Location X Sample Location & .41 & .522 & .002 \\
\hline
\end{tabular}

Note. All effects are $d f=1,226$

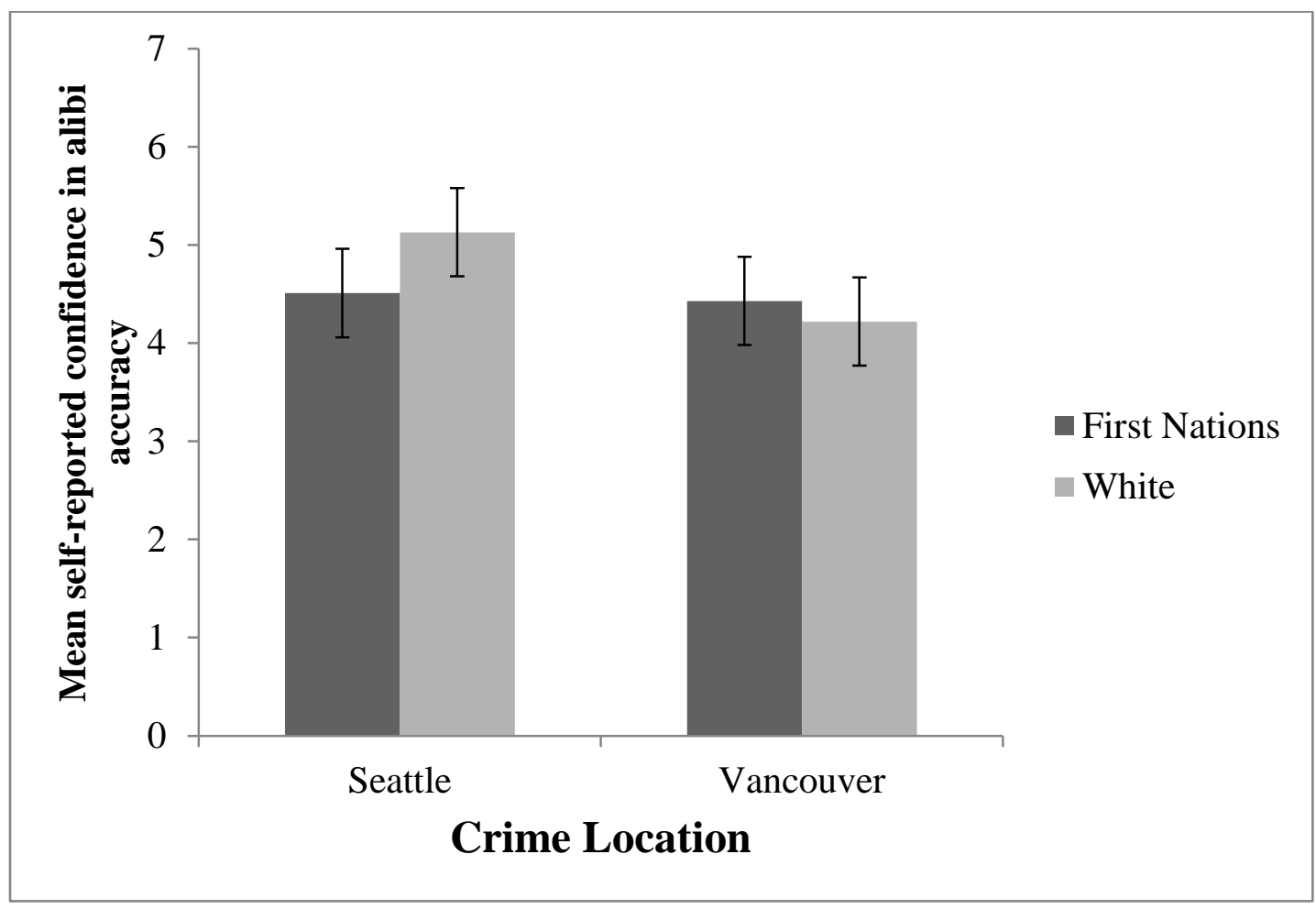

Figure 9. Participants' mean self-reported confidence in their ratings of the accuracy of the defendant's alibi.

Strength of the evidence against the defendant. When rating the strength of the evidence against the defendant, the only significant effect was a significant interaction between Crime Location and Defendant Race (see Table 44). As with participants' confidence in their ratings of 
alibi accuracy, follow-up tests indicated that when the crime was set in Seattle, the evidence was rated as stronger when the defendant was First Nations than when he was White, $t(78.33)=2.69$, $p=.009, d=0.55$, but there were no significant effects of Defendant Race when the crime was set in Vancouver, $t(122)=-.90, p=.370, d=-0.16$ (see Figure 10). It is also worth noting that, on average, participants rated the evidence against the defendant as being quite weak. Similar to the results of participants' confidence in their alibi accuracy ratings, the interaction appears to be due to responses in the White defendant in Seattle condition.

Table 44

ANOVA results for participants' ratings of the strength of the evidence against the defendant

\begin{tabular}{lccc}
\hline Effect & $F$ & $p$ & $\eta_{\mathrm{p}}^{2}$ \\
\hline Defendant Race & .90 & .344 & .004 \\
Crime Location & 3.06 & .081 & .013 \\
Sample Location & .94 & .335 & .004 \\
Defendant Race X Crime Location & 5.39 & .021 & .023 \\
Defendant Race X Sample Location & .81 & .370 & .004 \\
Crime Location X Sample Location & .60 & .439 & .003 \\
Defendant Race X Crime Location X Sample Location & .03 & .869 & $<.001$ \\
\hline
\end{tabular}

Note. All effects are $d f=1,227$

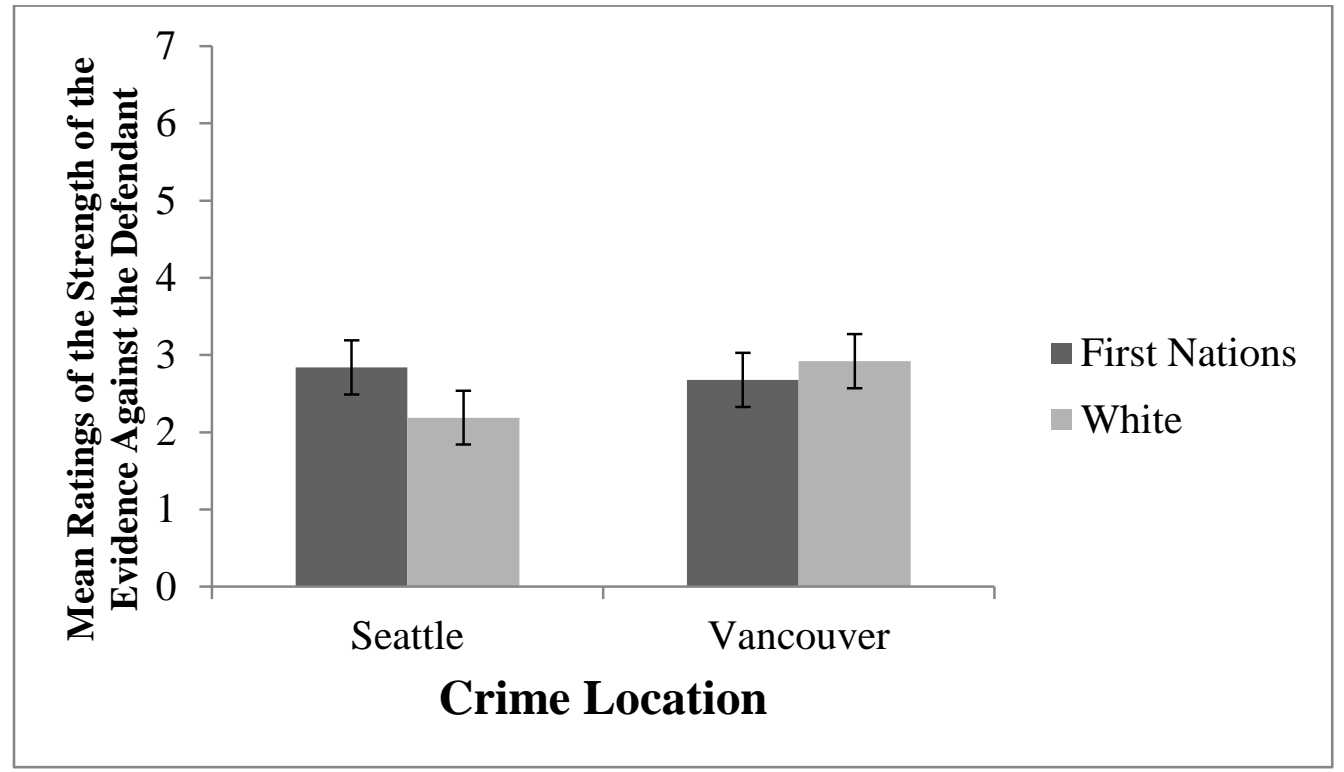

Figure 10. Participants' mean ratings of the strength of the evidence against the defendant. 
Alibi classification. Participants were all asked to determine whether the defendant's alibi was True (i.e., honest and accurate), Fabricated (i.e., dishonest and inaccurate), or Mistaken (i.e., honest and inaccurate). Overall, the majority of participants in both samples (77\%) indicated that the alibi was true (see Table 45). For participants who thought that the alibi was inaccurate, the majority believed that this was due to deliberate deception (i.e., intentional fabrication) rather than an error. The general pattern of responses - true being the most common response, mistaken being the least common response - was consistent with the data from Experiments 1 and 2. However, the percentage of participants who believed that the alibi was true was markedly higher in Experiment 3 than in the other two experiments (Experiment 1: 58.8\%; Experiment 2: 43.1\%; Experiment 3: 77.0\%). This is not surprising, given the notable differences in the materials between the two studies. In particular, participants in the first two experiments received more information overall, and more information about the suspect's alibi. Table 45

Participants' classifications of the defendant's alibi as percentages

\begin{tabular}{lccc}
\hline & Canadian & American & Total \\
\hline True & $77.3 \%$ & $76.6 \%$ & $77.0 \%$ \\
Fabricated & $18.0 \%$ & $18.7 \%$ & $18.3 \%$ \\
Mistaken & $4.7 \%$ & $4.7 \%$ & $4.7 \%$ \\
\hline
\end{tabular}

To determine whether Defendant Race, Crime Location, and Sample Location affected participants' classification of the alibi, a multinomial logistic regression was computed with all independent variables included as factors. As in Experiments 1 and 2, Fabricated as chosen as the reference category for the outcome variable because the comparison between True and Mistaken was not of primary theoretical interest. In this initial analysis, two cells showed zero frequencies. As the responses clearly do not differ substantially between the Canadian and 
American samples - an assertion that is supported by non-significant effects in the initial regression - the analysis was recomputed with only Defendant Race and Crime Location. Removing the Sample Location independent variable resulted in all cells having non-zero frequencies. The key result, as detailed below, was that participants were 2.38 times more likely to classify the alibi as True, rather than Fabricated, when the defendant was White (True: 84.3\%; Fabricated: 12.4\%) than when he was First Nations (True: 69.3\%; Fabricated: 24.6\%).

Non-significant Pearson, $\chi^{2}(2)=.39, p=.823$, and deviance, $\chi^{2}(2)=.39, p=.824$, values in the goodness-of-fit tests suggest that the model is a good fit for the data, though the model fit test was also non-significant, $\chi^{2}(4)=7.92, p=.095$, meaning that the final model does not fit the data significantly better than does the intercept-only model. This is consistent with the logit comparing the Fabricated and Mistaken categories; no significant effects were found for Crime Location, $b=41, S E=.68$, Wald $=5.08, p=.544$, Odds Ratio $=1.51$, or for Defendant Race, $b=$ $-.03, S E=.71$, Wald $=.002, p=.966$, Odds Ratio $=.97$. For the logit comparing True and Fabricated classifications, a significant effect was found for Defendant Race, $b=-.87, S E=.35$, Wald $=6.09, p=.014$, Odds Ratio $=.42$ However, the non-significant test of model fit means that this result may not be generalizable beyond the current sample. The effect of Crime Location was not significant, $b=.06, S E=.35$, Wald $=.03, p=.857$, Odds Ratio $=1.07$.

Likelihood of defendant guilt. As with earlier analyses, the only significant effect for likelihood of defendant guilt was an interaction between Crime Location and Defendant Race (see Table 46). Follow-up tests indicated that when the crime was set in Seattle, the likelihood that the defendant was guilty was rated as higher when he was First Nations than when he was White, $t(109)=4.11, p<.001, d=0.85$, but Suspect Race had no significant effect when the crime was set in Vancouver, $t(122)=-1.43, p=.156, d=-0.30$ (see Figure 11). Mean ratings in 
general indicated that participants were not particularly convinced that the defendant was guilty.

Table 46

ANOVA results for participants' ratings of the likelihood that the defendant is guilty

\begin{tabular}{lccc}
\hline Effect & $F$ & $p$ & $\eta_{\mathrm{p}}^{2}$ \\
\hline Defendant Race & 2.53 & .113 & .011 \\
Crime Location & 1.75 & .188 & .008 \\
Sample Location & .40 & .529 & .002 \\
Defendant Race X Crime Location & 12.94 & $<.001$ & .054 \\
Defendant Race X Sample Location & .68 & .412 & .003 \\
Crime Location X Sample Location & .009 & .926 & $<.001$ \\
Defendant Race X Crime Location X Sample Location & .66 & .419 & .003 \\
\hline
\end{tabular}

Note. All effects are $d f=1,227$

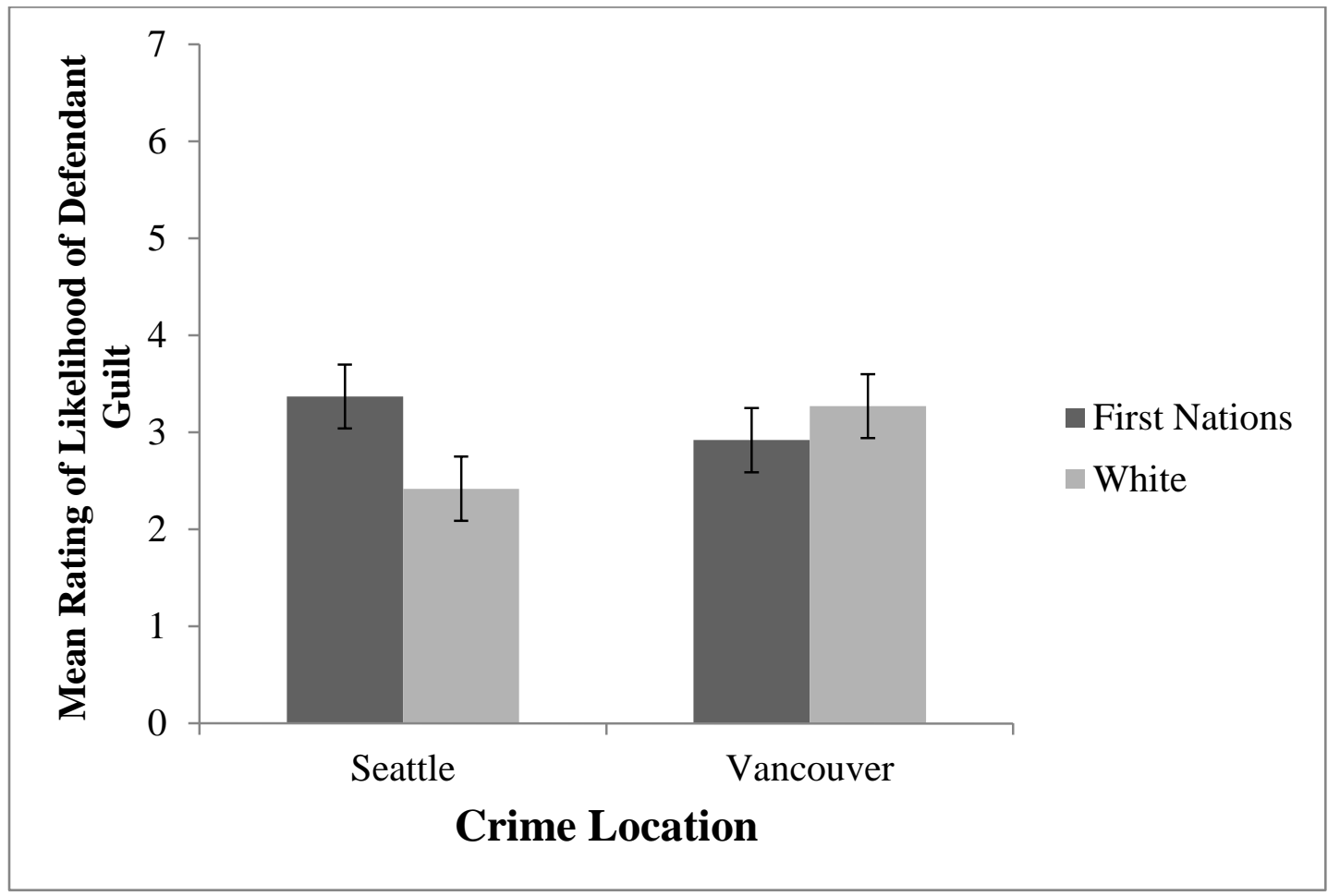

Figure 11. Participants' mean ratings of the likelihood that the defendant is guilty.

Verdict choice. Overall, 29 participants (12.3\%) provided guilty verdicts; 19 of these were from Canadian participants (14.8\% of Canadian participants) and 10 were from American participants $(9.3 \%)$ of American participants. The only significant effect was an interaction 
between Crime Location and Sample (see Table 47). For Canadian participants, more guilty verdicts were assigned when the crime was set in Seattle (19.0\%) than when it was set in Vancouver $(10.8 \%)$, but this difference was not statistically significant in the follow-up test, $\chi^{2}(1)=1.74, p=.220, \varphi=.116$. For the American sample, the chi-square is not reliable because one of the four cells has an expected count of less than 5. Descriptively, eight of the 10 guilty verdicts reported by American participants were from the Vancouver condition, and only two guilty verdicts were given when the crime was set in Seattle, consistent with what would be predicted for in-group bias.

Table 47

ANOVA results for participants' verdict choice

\begin{tabular}{lccc}
\hline Effect & $F$ & $p$ & $\eta_{\mathrm{p}}^{2}$ \\
\hline Defendant Race & 2.71 & .101 & .012 \\
Crime Location & .01 & .935 & $<.001$ \\
Sample Location & 1.86 & .174 & .008 \\
Defendant Race X Crime Location & .78 & .377 & .003 \\
Defendant Race X Sample Location & 2.75 & .099 & .012 \\
Crime Location X Sample Location & 4.20 & .042 & .018 \\
Defendant Race X Crime Location X Sample Location & .13 & .713 & .001 \\
\hline
\end{tabular}

Note. All effects are $d f=1,227$

In-group bias and non-legal judgments. In addition to their judgments about legal aspects of the case, participants rated the defendant on a number of other dimensions (e.g., similarity, liking, feelings if the defendant was a coworker). Again, interactions between sample location and crime location could constitute evidence for in-group identification. Additionally, more positive ratings of the White defendant than the First Nations defendant could indicate racial in-group bias, as the vast majority of the sample from both locations was White. 2 (Defendant Race: First Nations, White) X 2 (Crime Location: Seattle, Vancouver) X 2 (Sample: Canadian, American) ANOVAs were conducted for all dependent variables. Significant effects 
were found for five of nine of the dependent measures and will be discussed below. Nonsignificant effects were found for four measures; statistical results for these are available in Appendix FF.

General similarity. Participants rated how similar they believed they were to the defendant in general. Results are presented in Table 48. The only significant effect was that participants rated themselves as significantly more similar to the defendant when he was White $(M=2.63, S D=1.30)$ than when he was First Nations $(M=2.18, S D=1.31)$. The relatively low means indicate that participants generally believed that they were quite dissimilar to the defendant. Consequently, a floor effect may be obscuring genuine effects of Crime Location and Sample.

Table 48

ANOVA results for participants' ratings of how similar they are in general to the defendant

\begin{tabular}{lccc}
\hline Effect & $F$ & $p$ & $\eta_{\mathrm{p}}^{2}$ \\
\hline Defendant Race & 6.95 & .009 & .030 \\
Crime Location & 1.06 & .305 & .005 \\
Sample Location & .66 & .417 & .003 \\
Defendant Race X Crime Location & 2.37 & .125 & .010 \\
Defendant Race X Sample Location & .51 & .476 & .002 \\
Crime Location X Sample Location & 1.63 & .203 & .007 \\
Defendant Race X Crime Location X Sample Location & .22 & .642 & .001 \\
\hline
\end{tabular}

Note. All effects are $d f=1,226$

Similarity of personality. Participants also rated how similar they were to the defendant in terms of personality. The only significant effect was an interaction between Defendant Race and Crime Location (see Table 49 and Figure 12). Follow-up tests showed that when the crime was set in Vancouver, participants rated their personalities as being significantly more similar to the defendant when he was White then when he was First Nations, $t(122)=2.82, p=.006, d=$ 0.50. This effect of Defendant Race was not found when the crime was set in Seattle, $t(109)=-$ 
$.59, p=.555, d=-0.11$.

Table 49

ANOVA results for participants' ratings of how similar their personalities are to the defendant.

\begin{tabular}{lccc}
\hline Effect & $F$ & $p$ & $\eta_{\mathrm{p}}^{2}$ \\
\hline Defendant Race & 2.05 & .153 & .009 \\
Crime Location & .16 & .695 & .001 \\
Sample Location & .42 & .520 & .002 \\
Defendant Race X Crime Location & 5.34 & .022 & .023 \\
Defendant Race X Sample Location & .03 & .854 & $<.001$ \\
Crime Location X Sample Location & 1.28 & .260 & .006 \\
Defendant Race X Crime Location X Sample Location & .04 & .843 & $<.001$ \\
\hline
\end{tabular}

Note. All effects are $d f=1,227$

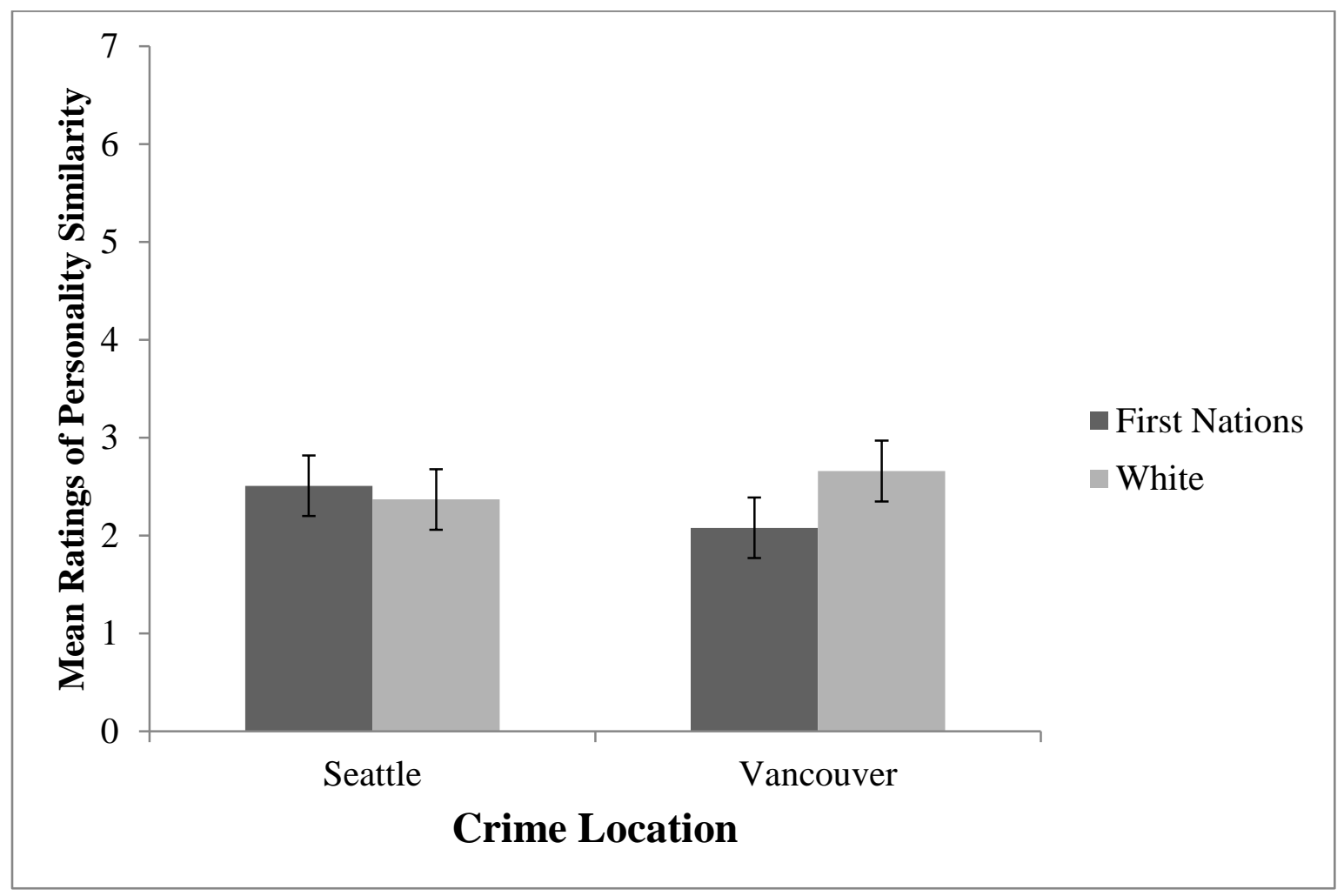

Figure 12. Participants' ratings of how similar they are in terms of personality to the defendant as a function of Crime Location and Defendant Race.

Differences in activities. Participants indicated how different they were from the defendant in terms of activities they enjoy engaging in. There was a significant main effect of Defendant Race and a marginally significant interaction between Defendant Race and Sample 
(see Table 50). When the defendant was First Nations $(M=4.74, S D=1.74)$, participants believed that the activities they enjoy are less similar to the activities that he enjoys compared to when the defendant was White $(M=4.24, S D=1.58)$. Follow-up independent-samples $t$-tests to probe the marginally significant interaction between Crime Location and Sample showed no significant differences between the Vancouver and Seattle settings for either the Canadian, $t(126)$ $=-1.46, p=.148, d=-0.26$, or American samples, $t(105)=1.12, p=.266, d=0.22$ (see Figure 13). The significant interaction is likely due to opposite patterns being observed in the two samples, such that Canadians rated themselves as more dissimilar from the defendant from Vancouver than the defendant from Seattle and Americans rated themselves as more dissimilar from the defendant from Seattle than the defendant from Vancouver. This is the opposite of what would be expected with in-group bias, though it is possible that participants did not read the item carefully and were rating similarity instead.

Table 50

ANOVA results for participants' ratings of how different the activities they enjoy are from the activities the defendant enjoys

\begin{tabular}{lccc}
\hline Effect & $F$ & $p$ & $\eta_{\mathrm{p}}^{2}$ \\
\hline Defendant Race & 5.54 & .019 & .024 \\
Crime Location & .03 & .872 & $<.001$ \\
Sample Location & .13 & .722 & .001 \\
Defendant Race X Crime Location & .15 & .703 & .001 \\
Defendant Race X Sample Location & .13 & .719 & .001 \\
Crime Location X Sample Location & 3.82 & .052 & .017 \\
Defendant Race X Crime Location X Sample Location & .49 & .483 & .002 \\
\hline
\end{tabular}

Note. All effects are $d f=1,227$ 


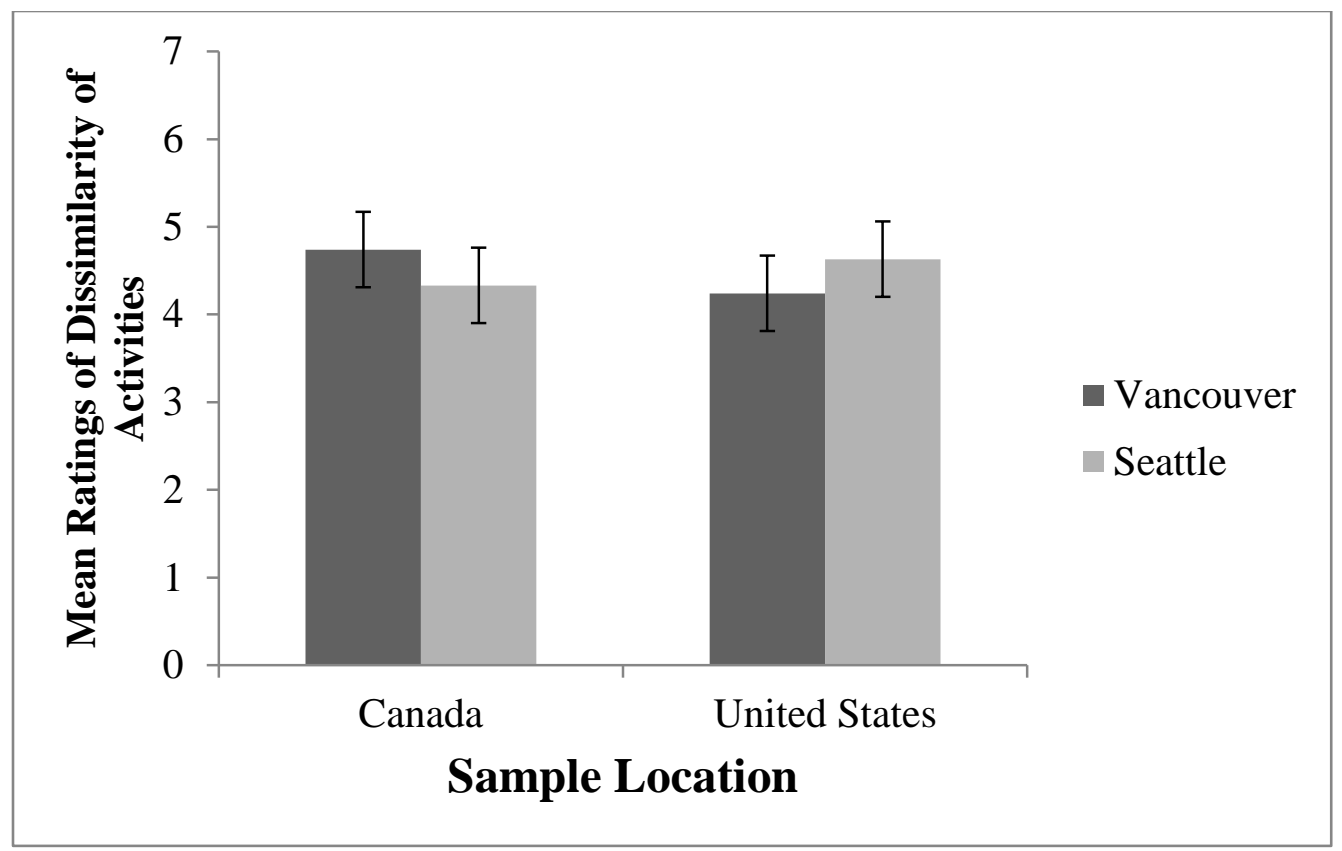

Figure 13. Comparison of Canadian and American participants' ratings of the dissimilarity of the activities that they and the First Nations or White defendant enjoy.

Friendship likelihood. Participants also rated the likelihood that they would become friends with the defendant if they ever met him. The only potentially noteworthy effect is a marginally significant main effect of Sample (see Table 51). American participants $(M=2.98$, $S D=1.30$ ) may believe that they are somewhat more likely to become friends with the defendant than Canadian participants $(M=2.65, S D=1.26)$. However, the relatively low means suggest that participants think it is relatively unlikely that they would become friends with the defendant, generally.

Is the defendant a good or bad person? Finally, participants evaluated the defendant as a good or bad person, overall, on a scale from 1 (A very bad person) to 11 (A very good person), with the midpoint labeled (Neither good nor bad). The only significant effect is for Defendant Race (see Table 52). Participants rated the defendant as neutral when he was White $(M=6.06$, $S D=1.60)$, but as a somewhat bad person when he was First Nations $(M=5.50, S D=1.60)$. 
Table 51

ANOVA results for participants' ratings of the likelihood of them becoming friends with the defendant

\begin{tabular}{lccc}
\hline Effect & $F$ & $p$ & $\eta_{\mathrm{p}}^{2}$ \\
\hline Defendant Race & 1.33 & .251 & .006 \\
Crime Location & 1.24 & .266 & .005 \\
Sample Location & 3.45 & .064 & .015 \\
Defendant Race X Crime Location & .77 & .382 & .003 \\
Defendant Race X Sample Location & .92 & .337 & .004 \\
Crime Location X Sample Location & $<.001$ & .988 & $<.001$ \\
Defendant Race X Crime Location X Sample Location & 2.29 & .131 & .010 \\
\hline
\end{tabular}

Note. All effects are $d f=1,226$

Table 52

ANOVA results for participants' ratings of whether the defendant is a good or bad person overall

\begin{tabular}{lccc}
\hline Effect & $F$ & $p$ & $\eta_{\mathrm{p}}^{2}$ \\
\hline Defendant Race & 7.56 & .006 & .032 \\
Crime Location & .56 & .457 & .002 \\
Sample Location & .42 & .518 & .002 \\
Defendant Race X Crime Location & .45 & .503 & .002 \\
Defendant Race X Sample Location & .28 & .601 & .001 \\
Crime Location X Sample Location & .02 & .904 & $<.001$ \\
Defendant Race X Crime Location X Sample Location & .85 & .358 & .004 \\
\hline
\end{tabular}

Note. All effects are $d f=1,226$

Summary of effects for non-legal dependent variables. In all, the effects of Defendant

Race, Crime Location, and Sample were investigated for nine dependent variables. For four of these, significantly less favourable ratings were provided for the First Nations defendant than for the White defendant, though ratings were not particularly positive for either defendant.

Additionally, Canadian participants rated themselves as less likely to become friends with the defendant than American participants, though this was one of only two significant effects for Sample, the other effect being an interaction in which the effect of Defendant Race was only observed for the Canadian sample. Finally, for four dependent measures, no significant effects 
were found for any independent variables.

The influence of participants' general feelings about First Nations and White people on their legal judgments. As with Experiment 2, participants' ratings about First Nations and White people were generally very positive and the distributions were markedly skewed to the left. Nonparametric tests indicated that there were no significant differences in the medians between the Canadian and American samples for ratings about First Nations, $p=.216$, or White people, $p=.257$. For perceptions of First Nations people, the mean rating was $75.75(S D=$ $22.99, M d n=80.0$ ) and for participants' ratings about White people, the mean rating was 77.28 $(S D=21.09, M d n=87.0)$. Participants' ratings of their feelings about First Nations and White people were significantly correlated, $r=.665, p<.001$ (see Figure 14).

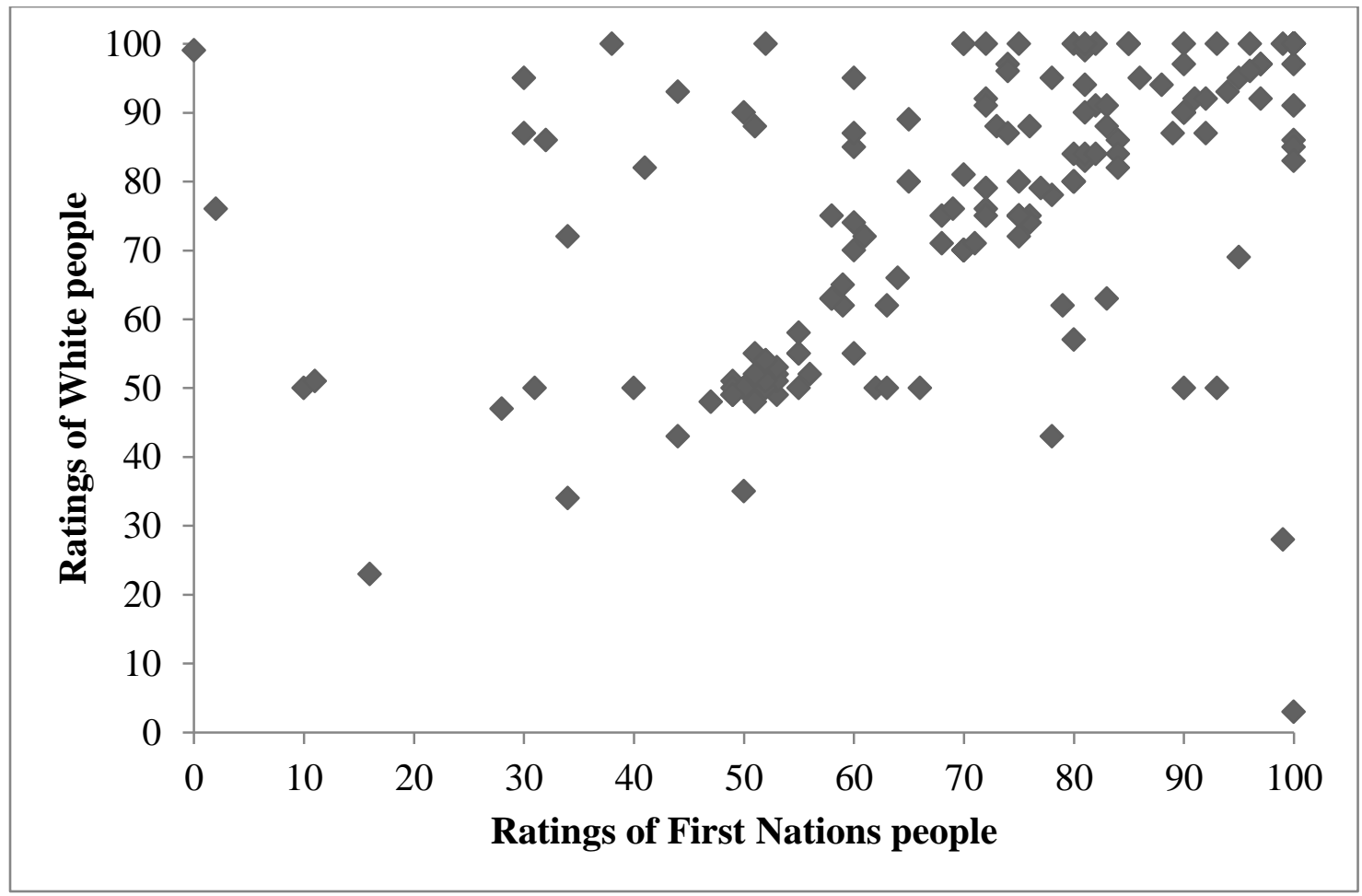

Figure 14. Correlation between participants' general feelings about First Nations and White people. 
Given the results of Experiment 2, it was expected that participants' general feelings about one group would predict their responses to the defendant such that more negative feelings would be related to ratings that were less favourable to the defendant. To test this hypothesis, regressions were computed separately for participants who read about the First Nations defendant and those who read about the White defendant. The regressions were computed twice. In the first model, only the rating for the defendant's group was included (e.g., general feelings about First Nations predicting responses about the First Nations defendant). In the second model, both ratings were included to determine whether any effects identified in the first model are still found when ratings for the other group are controlled. Statistical results are presented in Tables 53 and 54.

Two general patterns can be discerned from the data. First, participants' judgments about White defendants are unrelated to their general feelings about White or First Nations people. There was only one significant simple regression model—more favourable feelings about White people were associated with higher estimates of the strength of the evidence against the defendant —and this counterintuitive result disappeared in the multiple regression model where general feelings about First Nations people were controlled. The second general pattern is that more positive feelings about First Nations people were associated with more favourable outcomes for the First Nations defendant for ratings of statement honesty, confidence in statement honesty, alibi accuracy, and confidence in alibi accuracy. However, the models for evidence strength and likelihood of guilt were all non-significant. 
Table 53

Simple and multiple linear regression models using participants' general feelings about White and/or First Nations people to predict their judgments about White or First Nations suspects

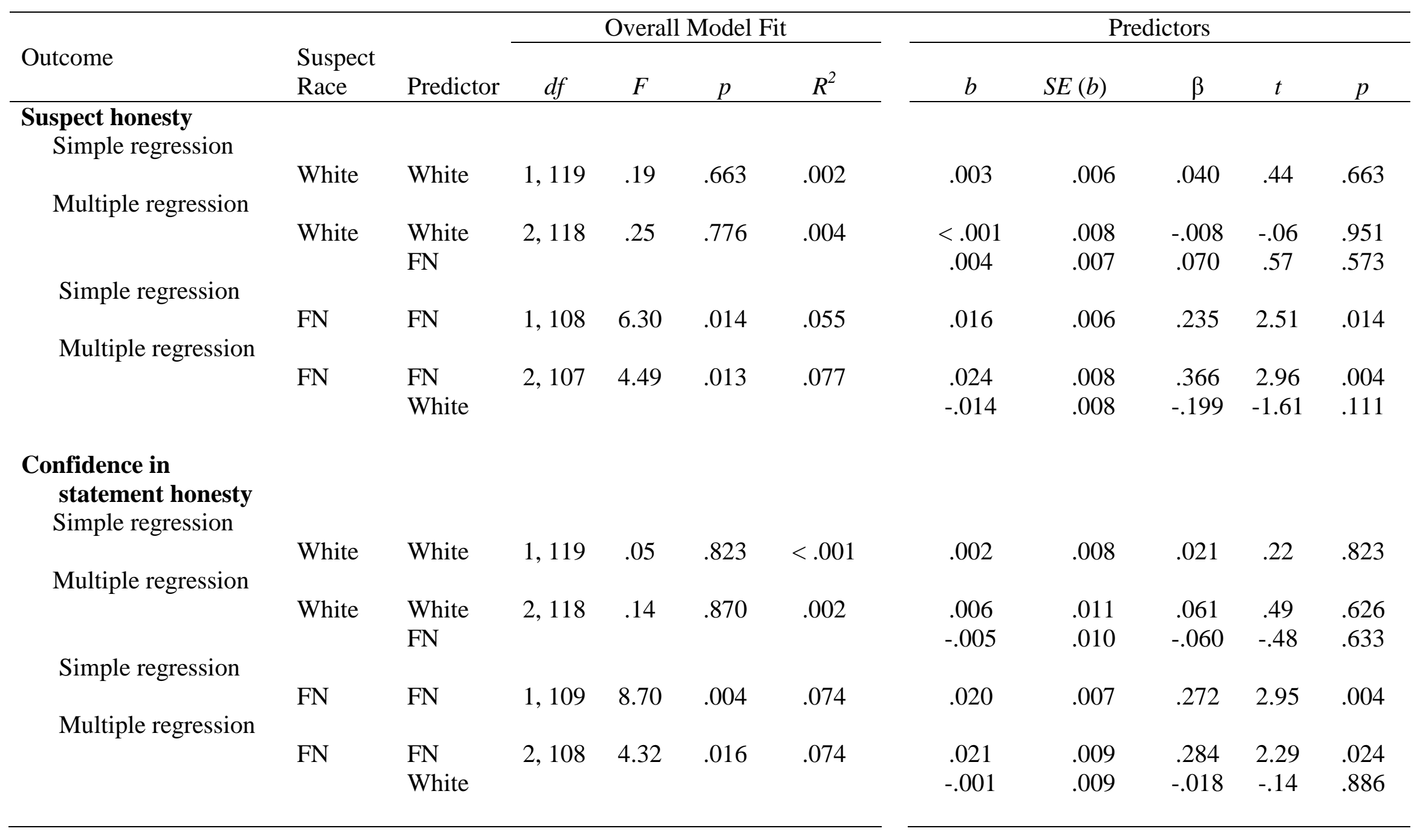




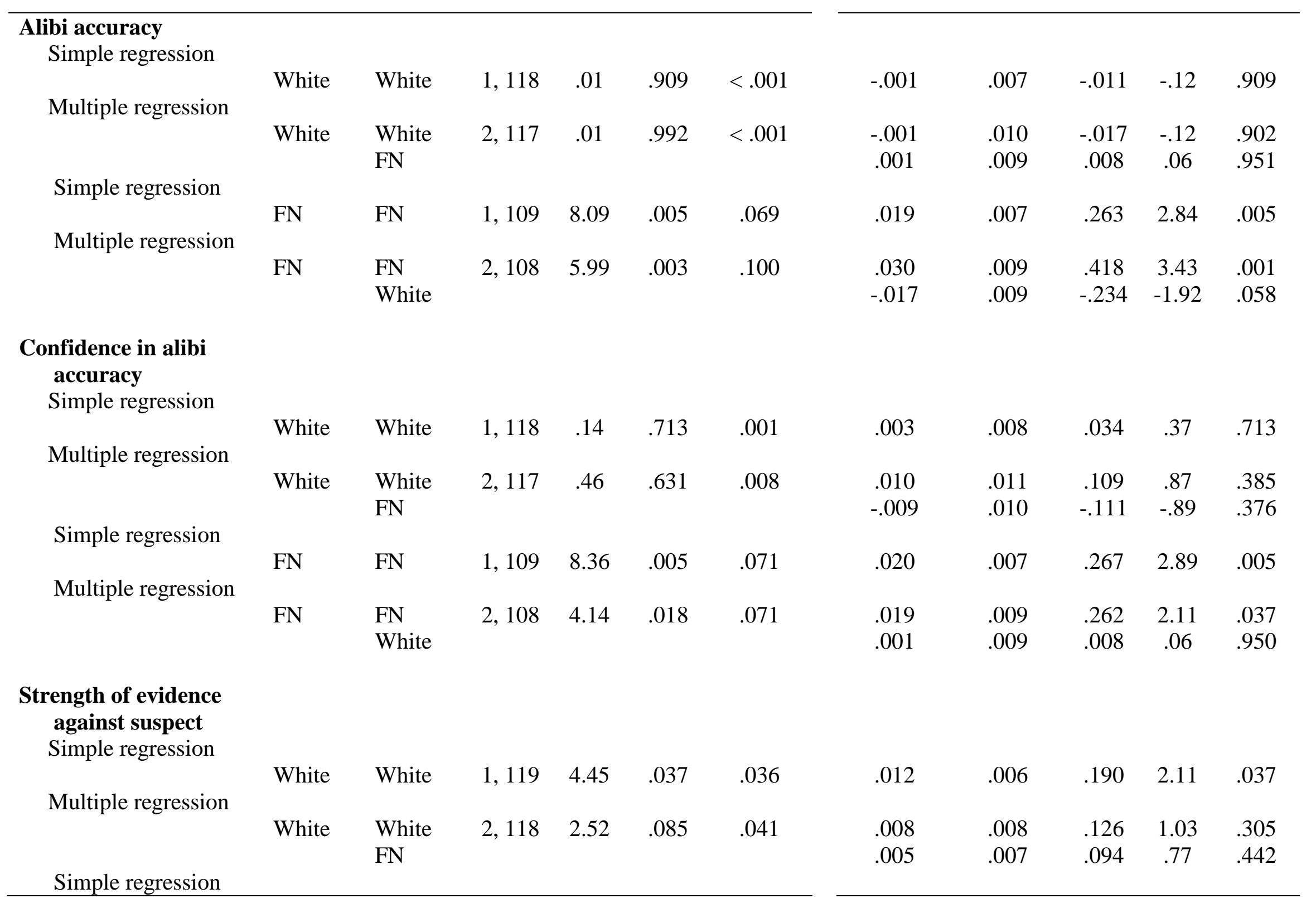




\begin{tabular}{|c|c|c|c|c|c|c|c|c|c|c|c|}
\hline \multirow{2}{*}{ Multiple regression } & FN & $\mathrm{FN}$ & 1,109 & .30 & .584 & .003 & .003 & .006 & .052 & .55 & .584 \\
\hline & $\mathrm{FN}$ & $\begin{array}{l}\text { FN } \\
\text { White }\end{array}$ & 2,108 & .39 & .676 & .007 & $\begin{array}{c}.007 \\
-.006\end{array}$ & $\begin{array}{l}.008 \\
.008\end{array}$ & $\begin{array}{r}.112 \\
-.089\end{array}$ & $\begin{array}{c}.87 \\
-.70\end{array}$ & $\begin{array}{l}.385 \\
.487\end{array}$ \\
\hline \multicolumn{12}{|l|}{$\begin{array}{l}\text { Likelihood of suspect } \\
\text { guilt } \\
\text { Simple regression }\end{array}$} \\
\hline Multiple regression & White & White & 1,119 & 1.44 & .232 & .012 & .007 & .006 & .109 & 1.20 & .232 \\
\hline Multiple regression & White & $\begin{array}{l}\text { White } \\
\text { FN }\end{array}$ & 2,118 & 1.01 & .367 & .017 & $\begin{array}{l}.003 \\
.005\end{array}$ & $\begin{array}{l}.008 \\
.007\end{array}$ & $\begin{array}{l}.045 \\
.095\end{array}$ & $\begin{array}{l}.37 \\
.77\end{array}$ & $\begin{array}{l}.715 \\
.446\end{array}$ \\
\hline \multirow{2}{*}{ Multiple regression } & $\mathrm{FN}$ & $\mathrm{FN}$ & 1,109 & 1.92 & .166 & .018 & -.008 & .006 & -.132 & -1.39 & .166 \\
\hline & $\mathrm{FN}$ & $\begin{array}{l}\text { FN } \\
\text { White }\end{array}$ & 2,108 & 1.04 & .356 & .019 & $\begin{array}{c}-.010 \\
.003\end{array}$ & $\begin{array}{l}.008 \\
.008\end{array}$ & $\begin{array}{l}-.166 \\
.050\end{array}$ & $\begin{array}{c}-1.30 \\
.40\end{array}$ & $\begin{array}{l}.196 \\
.693\end{array}$ \\
\hline
\end{tabular}


Table 54

Simple and multiple binary logistic regression models using participants' general feelings about White and/or First Nations people to predict their judgments about White or First Nations suspects

\begin{tabular}{|c|c|c|c|c|c|c|c|c|c|c|c|}
\hline \multirow[b]{2}{*}{ Outcome } & \multirow[b]{2}{*}{$\begin{array}{l}\text { Suspect } \\
\text { Race }\end{array}$} & \multirow[b]{2}{*}{ Predictor } & \multicolumn{4}{|c|}{ Overall Model Fit } & \multicolumn{5}{|c|}{ Predictors } \\
\hline & & & $d f$ & $\chi^{2}$ & $p$ & $R^{2}$ & $b$ & $S E(b)$ & Wald & $p$ & $\begin{array}{l}\text { Odds } \\
\text { Ratio }\end{array}$ \\
\hline \multicolumn{12}{|l|}{$\begin{array}{l}\text { Verdict choice } \\
\text { Simnle reoression }\end{array}$} \\
\hline Simple regression & White & White & 1 & 2.29 & .130 & .043 & -.029 & .021 & 1.84 & .175 & .971 \\
\hline tvicut & White & $\begin{array}{l}\text { White } \\
\text { FN }\end{array}$ & 2 & 2.31 & .314 & .044 & $\begin{array}{l}-.027 \\
-.003\end{array}$ & $\begin{array}{l}.026 \\
.019\end{array}$ & $\begin{array}{l}1.03 \\
.02\end{array}$ & $\begin{array}{l}.309 \\
.887\end{array}$ & $\begin{array}{l}.997 \\
.997\end{array}$ \\
\hline Simple regression & $\mathrm{FN}$ & FN & 1 & .007 & .935 & $<.001$ & 1.64 & .937 & 3.05 & .081 & 5.14 \\
\hline & $\mathrm{FN}$ & $\begin{array}{l}\text { FN } \\
\text { White }\end{array}$ & 2 & .007 & .997 & $<.001$ & $\begin{array}{c}.001 \\
<.001\end{array}$ & $\begin{array}{l}.016 \\
.016\end{array}$ & $\begin{array}{l}.005 \\
<.001\end{array}$ & $\begin{array}{l}.944 \\
.989\end{array}$ & $\begin{array}{l}1.001 \\
1.000\end{array}$ \\
\hline
\end{tabular}

Note. The Nagelkerke $R^{2}$ is reported. Also, note that verdict choice is coded as $1=$ Guilty, $2=$ Innocent. 
Authoritarianism. Another primary focus of Experiment 3 was to investigate the relationships between Authoritarianism and legal decisions with a community sample. Only one participant failed to complete the ACT (see Appendix GG for details of psychometric analyses). The alpha levels, inter-item correlations, and item-total correlations were excellent and no items were removed. A wide range of scores were observed and, as in Experiments 1 and 2, mean scores generally fell slightly below the midpoint. Scores from American participants were consistently significantly higher than scores from Canadian participants. Americans' scores were also more variable than Canadians' for the ACT total score as well as scores on the Conservatism and Traditionalism subscales. Scores on the ACT did not vary across experimental conditions, aside from one interaction between Crime Location and Defendant Race for participants' responses on the Authoritarianism subscale, $F(1,227)=6.78, p=.010, \eta_{\mathrm{p}}^{2}=.03$. It seems as though when the crime was set in Seattle, participants' Authoritarianism scores were higher when the suspect was White than Indigenous, but when the crime was set in Vancouver, the reverse pattern was observed. Differences in means within the two Crime Location conditions were approximately 3 points.

A series of simple regressions were computed to investigate whether participants' scores on the ACT and its subscales were significantly associated with their legal judgments. Sample was not included as a variable in these models because the ANOVAs conducted earlier did not show consistent significant differences between the two samples on these outcome measures. The interaction between the two variables was not included because there is no strong rationale to expect that Authoritarianism operates differently in Canada and the United States.

In total, eight significant models were observed (see Tables 55 and 56), with the Authoritarianism subscale and total ACT scores being the most useful predictors. Interestingly, 
Table 55

Statistical results for linear regression models using ACT total and subscale scores to predict participants' responses on dependent measures

\begin{tabular}{|c|c|c|c|c|c|c|}
\hline Outcome & Predictor & $b$ & $S E(b)$ & $\beta$ & $R^{2}$ & $p$ \\
\hline \multicolumn{7}{|c|}{ Defendant honesty } \\
\hline & $\mathrm{ACT}$ & -.006 & .004 & -.091 & .008 & .168 \\
\hline & Conservatism & -.011 & .011 & -.066 & .004 & .316 \\
\hline & Traditionalism & -.011 & .011 & -.065 & .004 & .324 \\
\hline & Authoritarianism & -.020 & .012 & -.111 & .012 & .090 \\
\hline \multicolumn{7}{|c|}{$\begin{array}{l}\text { Confidence in defendant } \\
\text { honesty }\end{array}$} \\
\hline & $\mathrm{ACT}$ & .009 & .005 & .105 & .011 & .109 \\
\hline & Conservatism & .017 & .014 & .080 & .006 & .225 \\
\hline & Traditionalism & .018 & .014 & .084 & .007 & .197 \\
\hline & Authoritarianism & .025 & .015 & .112 & .013 & .087 \\
\hline \multicolumn{7}{|c|}{ Alibi accuracy } \\
\hline & $\mathrm{ACT}$ & -.004 & .005 & -.053 & .003 & .421 \\
\hline & Conservatism & -.003 & .012 & -.017 & $<.001$ & .801 \\
\hline & Traditionalism & -.011 & .012 & -.061 & .004 & .354 \\
\hline & Authoritarianism & -.013 & .013 & -.065 & .004 & .324 \\
\hline \multicolumn{7}{|c|}{ Confidence in alibi accuracy } \\
\hline & $\mathrm{ACT}$ & .013 & .005 & .162 & .026 & .013 \\
\hline & Conservatism & .022 & .014 & .104 & .011 & .115 \\
\hline & Traditionalism & .027 & .014 & .131 & .017 & .046 \\
\hline & Authoritarianism & .043 & .014 & .191 & .037 & .003 \\
\hline \multicolumn{7}{|c|}{$\begin{array}{l}\text { Strength of evidence against } \\
\text { defendant }\end{array}$} \\
\hline & $\mathrm{ACT}$ & .009 & .004 & .144 & .021 & .027 \\
\hline & Conservatism & .017 & .011 & .103 & .011 & .117 \\
\hline & Traditionalism & .015 & .011 & .090 & .008 & .168 \\
\hline & Authoritarianism & .034 & .011 & .194 & .038 & .003 \\
\hline \multicolumn{7}{|c|}{ Likelihood of Guilt } \\
\hline & $\mathrm{ACT}$ & .004 & .004 & .069 & .005 & .290 \\
\hline & Conservatism & .006 & .011 & .040 & .002 & .544 \\
\hline & Traditionalism & .004 & .010 & .023 & .001 & .726 \\
\hline & Authoritarianism & .022 & .011 & .131 & .017 & .046 \\
\hline
\end{tabular}


Table 56

Statistical results for binary logistic regression models using ACT total and subscale scores to predict participants' verdict choice and petition signing decision

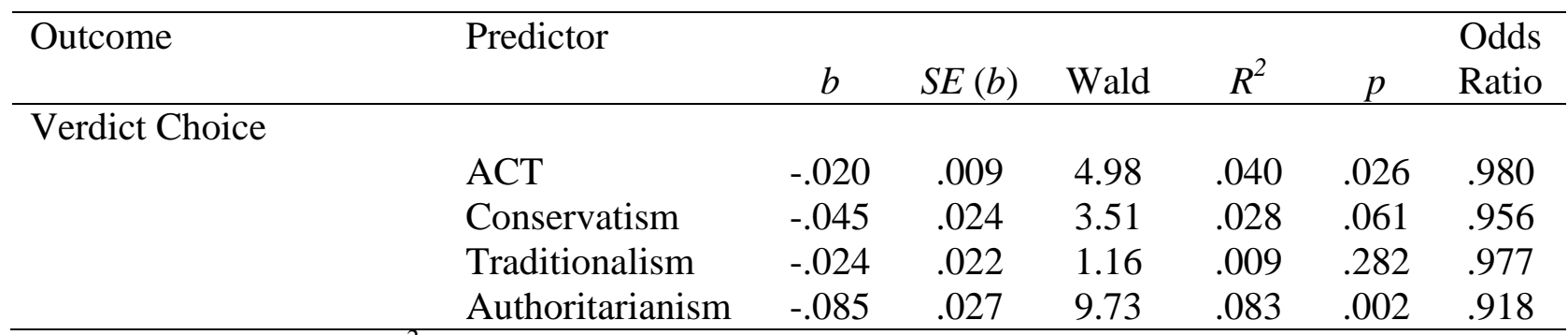

Note. The Nagelkerke $R^{2}$ is reported. Also, note that verdict choice is coded as $1=$ Guilty, $2=$ Innocent.

scores predicted judgments about broader issues (e.g., strength of evidence against the suspect,

likelihood of guilt, verdict choice) rather than specific ones (i.e., defendant honesty, alibi

accuracy). In all significant models, higher scores were associated with ratings that were less

favourable for the defendant. They also predicted participants' confidence in their judgment

about alibi accuracy; higher ACT totals, Traditionalism, and Authoritarianism scores were

associated with significantly higher confidence self-reports.

Religious orientation. Participants also completed a modified version of Hills et al.’s

(2005) Revised Religious Life Inventory (RLI-R) to investigate whether respondent's religious

orientation was related to their legal judgments. A high number of participants used the "Not

Applicable" option for most or all of their responses. For the most part, when participants chose

the "Not Applicable" option for one item, they chose it for the majority of the other items as

well. Thus, total scores on each subscale were computed by taking the mean of approximately

$80 \%$ or more of the items. Individuals who responded to fewer items than this did not receive a

score. Of a total of 235 participants, 120 received scores for the Extrinsic subscale, 120 received

scores for the Intrinsic subscale, and 138 received scores for the Quest subscale. No significant

differences in scores on any of the subscales were observed based on experimental condition, 
though there was a trend for scores on the Intrinsic subscale to be marginally higher for Canadian than American participants, $F(1,112)=3.74, p=.056, \eta_{\mathrm{p}}^{2}=.03$ (all other $F<2.455$, all other $p$ $>$.120).

Does level of endorsement of particular religious orientations predict legal judgments?

This study was also concerned with whether there was an association between religious orientation and legal judgments. It was hypothesized that higher endorsement of Extrinsic religiosity, particularly the need for status and security, would be associated with legal judgments that are less favourable to the defendant. Intrinsic orientation was expected to be related to legal judgments, but the association could be positive or negative. Finally, it was not clear whether or how Quest orientation would be related to the dependent measures.

To investigate these questions, a series of simple regression models were computed, using scores on the subscales of the RLI-R (Hills et al., 2005) to predict participant's legal judgments. No significant differences between the Canadian and American samples were found on any of the subscales (see Appendix GG). The alpha levels were all very good, and were similar to the alphas obtained by Hills et al. (2005). Additionally, the inter-item and item-total correlations did not show any problematic items. Only three significant models were identified (see Tables 57 and 58). Higher endorsement of items on the Quest subscale was associated with higher confidence in alibi accuracy judgments and lower ratings of the strength of the evidence against the suspect. As well, higher endorsement of items on the Intrinsic subscale was significantly related to higher estimates of the likelihood that the suspect was guilty. With the number of models computed and the fact that two of the significant models had $p$-values that were very close to .05 , it is necessary to consider that the significant results may represent a Type II error. If these are genuine effects, it appears that individuals who consider religion from 
Table 57

Statistical results for linear regression models using RLI-R subscale scores to predict participants' responses on dependent measures

\begin{tabular}{|c|c|c|c|c|c|c|}
\hline Outcome & Predictor & $B$ & $S E(b)$ & $\beta$ & $R^{2}$ & $p$ \\
\hline \multicolumn{7}{|c|}{ Defendant honesty } \\
\hline & Extrinsic & .026 & .086 & .028 & .001 & .763 \\
\hline & Intrinsic & -.063 & .057 & -.102 & .010 & .269 \\
\hline & Quest & .091 & .069 & .113 & .013 & .190 \\
\hline \multicolumn{7}{|c|}{$\begin{array}{l}\text { Confidence in defendant } \\
\text { honesty }\end{array}$} \\
\hline & Extrinsic & .051 & .097 & .048 & .002 & .599 \\
\hline & Intrinsic & -.045 & .063 & -.065 & .004 & .477 \\
\hline & Quest & .140 & .080 & .149 & .022 & .080 \\
\hline \multicolumn{7}{|c|}{ Alibi accuracy } \\
\hline & Extrinsic & .032 & .090 & .033 & .001 & .725 \\
\hline & Intrinsic & -.078 & .059 & -.122 & .015 & .186 \\
\hline & Quest & .110 & .075 & .125 & .016 & .147 \\
\hline \multicolumn{7}{|c|}{ Confidence in alibi accuracy } \\
\hline & Extrinsic & .037 & .099 & .034 & .001 & .711 \\
\hline & Intrinsic & -.048 & .064 & -.069 & .005 & .455 \\
\hline & Quest & .163 & .083 & .167 & .028 & .051 \\
\hline \multicolumn{7}{|c|}{$\begin{array}{l}\text { Strength of evidence against } \\
\text { defendant }\end{array}$} \\
\hline & Extrinsic & -.041 & .079 & -.047 & .002 & .607 \\
\hline & Intrinsic & .091 & .052 & .159 & .025 & .083 \\
\hline & Quest & -.140 & .064 & -.184 & .034 & .030 \\
\hline \multicolumn{7}{|c|}{ Likelihood of Guilt } \\
\hline & Extrinsic & -.007 & .080 & -.008 & $<.001$ & .934 \\
\hline & Intrinsic & .105 & .053 & .181 & .033 & .048 \\
\hline & Quest & -.123 & .068 & -.154 & .024 & .072 \\
\hline
\end{tabular}

Table 58

Statistical results for binary logistic regression model using RLI-R subscale scores to predict participants'verdict choice

\begin{tabular}{llcccccc}
\hline Outcome & Predictor & & & & & $\begin{array}{c}\text { Odds } \\
\text { Ratio }\end{array}$ \\
\hline Verdict Choice & & $b$ & $S E(b)$ & Wald & $R^{2}$ & $p$ & \\
& Extrinsic & .022 & .180 & 0.02 & $<.001$ & .901 & 1.023 \\
& Intrinsic & -.165 & .110 & 2.25 & .034 & .134 & .848 \\
& Quest & .144 & .143 & 1.007 & .014 & .316 & 1.155 \\
\hline
\end{tabular}

Note. The Nagelkerke $R^{2}$ is reported. Also, note that verdict choice is coded as $1=$ Guilty, $2=$ Innocent. 
a perspective of exploration (i.e., high Quest scores) are more likely to be confident when judging the accuracy of an alibi and are more likely to question the evidence that is brought against a defendant. The latter is consistent with the characterization of the Quest approach to religion, as it involves having reservations about the evidence implicating the defendant. However, it is somewhat counterintuitive that individuals who are very engaged in questioning religion are less likely to question themselves, in terms of their judgment of alibi accuracy. Finally, the significant model for Intrinsic motivation suggests that individuals who consider their religion as a core part of their self may be prone to judge others who do not live up to their own standards for behaviour.

\section{Do high-responders and low-responders on the RLI-R differ in their legal judgments?} As approximately half of the sample responded "Not Applicable" to the majority of items on the RLI-R, it was also of interest to examine whether there are differences between these LowResponders and participants who responded to most or all of the items on the RLI-R (i.e., HighResponders). High-Responders $(n=108)$ had scores on all three RLI-R subscales, LowResponders $(n=90)$ did not have scores on any of the RLI-R subscales. Participants $(n=37)$ who had scores for some, but not all of the subscales were excluded, as it was unclear which group was appropriate. No significant differences were observed between the High-Responder and Low-Responder groups for any of the continuous dependent measures, except for a nearly significant trend for High-Responders to be more confident in their ratings of defendant honesty than Low-Responders. There was also no significant association between the High-Responders and Low-Responders in rates of guilty verdicts, $\chi^{2}(1)=.51, p=.498, \varphi=.051$. Overall, there was no evidence that responses to legal matters differed depending on whether participants were religious or not (see Table 59). 
Table 59

Comparison of legal judgments between Religious and Non-Religious participants.

\begin{tabular}{lcccccc}
\hline Outcome & $d f$ & $t$ & $P$ & $d$ & $M 1(S D)$ & $M 2(S D)$ \\
\hline Defendant honesty & 195 & -.38 & .703 & -0.05 & $4.64(1.48)$ & $4.71(1.26)$ \\
Confidence in defendant honesty & 196 & 1.95 & .052 & 0.28 & $4.51(1.68)$ & $4.02(1.83)$ \\
Alibi accuracy & 195 & -.18 & .854 & -0.03 & $4.92(1.57)$ & $4.96(1.42)$ \\
Confidence in alibi accuracy & 195 & 1.58 & .115 & 0.23 & $4.76(1.69)$ & $4.37(1.76)$ \\
Strength of evidence against the & 196 & 1.49 & .138 & 0.21 & $2.70(1.37)$ & $2.42(1.27)$ \\
$\quad$ & & & & & & \\
$\quad$ Likelihondant & 195.66 & -.03 & .975 & 0.00 & $2.92(1.41)$ & $2.92(1.12)$ \\
\hline
\end{tabular}

Note. M1 refers to the High-Responder group and M2 refers to the Low-Responder group. The $d$ for likelihood of guilt ratings is noted as 0 , rather than $<.01$ because the two means were identical, and the difference between the two means is the dividend in the computation.

Qualitative analysis of participants' definitions of an “alibi." These data were treated as in Experiments 1 and 2. In the initial pass, the two coders provided identical codes to $77.0 \%$ of the participants' responses. In an additional $3.4 \%$ of cases, there was partial agreement. The more substantial disagreements in $19.6 \%$ of participants' responses was almost entirely in circumstances where the primary coder determined that the response emphasized the evidence supporting a narrative and the secondary coder assigned codes for a narrative that was supported by evidence. As the discrepancy was arguably due to the second coder misunderstanding the codes, the primary coder's codes were used.

Approximately half (47.7\%) of participants correctly reported that an alibi is a narrative that establishes that a suspect was elsewhere at the time of a crime and could not be the perpetrator (code 1 in codebook). An additional $12.8 \%$ of participants indicated that an alibi was a narrative about where someone was at a particular time, but did not reference a crime (code 2). A number of participants who made primary reference to narratives $(29.6 \%)$ also noted the need for or possibility of evidence to corroborate the alibi (code 11).

A notable number of participants $(28.5 \%)$ erroneously reported that the corroborating 
evidence - rather than the narrative itself - was the alibi (code 5). However, this occurred somewhat less frequently than it did in Experiments 1 and 2.The remaining codes were rarely assigned (i.e., to approximately 5\% or fewer of the responses). As in Experiments 1 and 2, no differences in judgments about the case were found between participants who indicated that an alibi was a narrative establishing innocence in a crime and participants who reported that an alibi was evidence (see Appendix HH).

\section{Discussion}

\section{The effects of defendant race, crime location, and sample location on legal}

judgments. The general pattern of results for participants' legal judgments was that when significant effects were found, responses that were less favourable for the defendant were provided when he was First Nations than when he was White (e.g., evidence against defendant is stronger, likelihood of guilt is higher), though in most cases, these effects were only found when the crime was set in Seattle. It must be noted that this effect was found for several, but not all, dependent measures. In general, there was no evidence of systematic differences between the Canadian and American samples, though a few significant effects were observed.

This interaction between Defendant Race and Crime Location was unexpected, and there are no obvious broad theoretical explanations for why differences in Defendant Race should be contingent on the city in which the crime took place. Much more likely is that the effect is due to the specific materials used in the study. In particular, when the crime was set in Vancouver, the defendant was referred to as "First Nations," whereas when the crime was set in Seattle, the term was replaced with "American Indian," to be more consistent with the terminology in general use in the United States. Indeed, this is the term used in demographic items in research. Somewhat different schemas may have been activated by the two terms. The term "Indian" to refer to 
Indigenous peoples is generally considered racist, though there is no agreement currently regarding whether "American Indian" is racist (e.g., Walbert, n.d.). Perhaps the use of the term "Indian" in the Seattle condition primed participants to make harsher judgments for the Indigenous defendant than the White defendant. In the Vancouver condition, use of a more neutral term-"First Nations"--would not have primed this same response. However, this remains an empirical question.

Participants' general feelings about White and First Nations people. Participants' general feelings about First Nations people fairly consistently affected their legal judgments when the defendant was First Nations, even when participants' general feelings about White people were controlled. As expected, more positive feelings about First Nations people were associated with case-related judgments that are more favourable for the defendant. Naturally, the opposite is also true: participants who had more negative views of First Nations people tended to give judgments that are less favourable for the defendant. In contrast, participants' general feelings about White people were not consistently associated with their legal judgments.

Interestingly, the significant effects for the First Nations defendant were found for the dependent measures that were relatively narrow in scope and related to the defendant's statement, and not for broader judgments, such as likelihood of guilt. In the Olson and Wells (2004) model, this would be the distinction between the Evaluation Phase, where the alibi itself is evaluated, and the Ultimate Evaluation Phase, where an ultimate determination is made about the guilt or innocence of the individual. Participants were given little information with which to evaluate the defendant's alibi accuracy or the honesty of his statement. The only corroboration was by motivated familiar others, and corroboration of this type is unlikely to affect judgments, relative to a no-corroboration control (Culhane \& Hosch, 2004; Hosch, Culhane, \& Howley, 
2005; Olson \& Wells, 2004). This suggests that participants viewed corroboration by a friend or family member as having low diagnosticity and did not weigh this information heavily when making their decisions. In the absence of other potentially useful information, participants may over-rely on their stereotypes about First Nations people. Although participants were not provided with much more information to make their judgments about the strength of the evidence against the suspect or the likelihood of his guilt, they did have relatively more relevant information (e.g., eyewitness report, fingerprint evidence), and, consequently, would presumably rely relatively less on their stereotypes when providing their ratings.

\section{The effect of defendant race, crime location, and sample location on non-legal}

judgments. Overall, participants did not believe they were particularly similar to the defendant and had somewhat negative views of the defendant. Also, participants, the majority of whom were White, rated themselves as significantly less similar to the defendant in general, in terms of personality, and in terms of what activities they enjoy when he was First Nations compared to when he was White. It is not surprising to see participants rating themselves as being more similar to an in-group member than to an out-group member. Finally, the First Nations defendant was also rated as a somewhat bad person, whereas the White defendant was rated neutrally. However, several other dependent measures did not show significant effects for Defendant Race.

In-group bias. Overall, there was not strong evidence to implicate in-group bias as affecting judgments in a legal context in the present study. Participants from the samples were not more likely to provide more lenient responses to the defendant who was of the same nationality (i.e., interaction between Crime Location and Sample Location). The one exception was that Canadian participants provided significantly more guilty verdicts for the American defendant, and, descriptively, it appears that the American participants provided more guilty 
verdicts for the Canadian defendant. However, very few guilty verdicts were provided, so an increase or decrease of only very few guilty verdicts could have a large effect on the results. Additionally, as described in the previous section, no consistent pattern of interactions between Sample Location and Crime Location were observed for participants' non-legal judgments. Taken together, this suggests that in-group bias did not affect the results of the present study.

Authoritarianism. Some evidence was found that Authoritarianism affects legal judgments in the direction hypothesized. In particular, scores on the Authoritarianism subscale were associated with responses that are less favourable for the defendant on some, but not all, dependent measures. With one exception, total scores on the ACT showed the same effects, likely due in large part to the Authoritarianism component. This is consistent with the results from Experiments 1 and 2 and with Cowan (2012), showing higher scores are related to harsher judgments. The results are also consistent with previous literature showing Authoritarianism is positively correlated with a greater number of guilty verdicts and convictions (Devine, Clayton, Dunford, Seying, \& Price, 2001; Narby, Cutler, \& Moran, 1993), longer recommended sentences (Devine et al., 2001), support for capital punishment (McKee \& Feather, 2008) and more skepticism of alibis (Cowan, 2012; Culhane, 2005). Still, there were a number of non-significant effects observed.

It is interesting that it is the Authoritarianism subscale, in particular, where significant effects were found. This subscale relates particularly to punishing transgressors and adopting a "tough-on-crime" type agenda. The link between these attitudes and determining that someone is guilty (i.e., likelihood of guilt, verdict choice) is clear. Individuals who are higher on this aspect of Authoritarianism are inclined to punish and, therefore, are setting the stage for punishment by determining that the defendant is likely guilty. Participants may justify this decision to 
themselves by also determining that the evidence against the suspect is relatively strong.

The content of the materials may have eliminated the possibility of many significant effects for Traditionalism or Conservatism. Aside from the accusation of murder, the information provided about the defendant was either neutral or positive. The defendant clearly was employed, as the article described him performing work as a handyman in the victim's apartment. His alibi included attending a concert with friends, which is arguably neutral. Essentially, notwithstanding his status as a defendant in a murder trial, no information suggested that the defendant violated traditional social norms or showed disrespect for legitimate authorities. Thus, there was little information in the materials to prime participants' views on Traditionalism and Conservatism.

Religious orientation. One novel aspect of the current study was investigating whether religiosity affects legal judgments, particularly those related to alibis. It was expected that individuals who scored higher on Extrinsic orientation would provide less favourable responses and that scores on Intrinsic orientation would be related - either positively or negatively — to participants' responses. Support for these hypotheses was not found; only three regression models were significant and two implicated the Quest subscale, for which there were no formal hypotheses.

Many participants in the sample do not appear to have identified as regular practicing members of a religious group (i.e., provided mostly "Not Applicable" responses). This finding is in contrast to results of the Canadian National Household Survey in 2011 (Statistics Canada, 2011) and data from the U.S. Census Bureau (2012) that indicated that the majority of Canadians and Americans self-identify as religious. It is possible that these individuals who were raised in a particular religious tradition — but do not currently practice — could self-identify as religious on a 
census and respond Not Applicable to items on the RLI-R, but this is an empirical question. There were no significant differences in legal judgments between participants who responded to the majority of the RLI-R items with ratings and participants who provided mostly "Not Applicable" responses. Taken together, these results suggest that religious orientation does not have a general, consistent influence on judgments related to legal issues.

Participants' definition of the term "alibi." Overall, approximately half of the participants correctly indicated that an alibi is narrative establishing that a suspect was somewhere other than a crime scene and, therefore, could not be the guilty party. Approximately a third of these also indicated that corroborating evidence would increase the credibility of the narrative. This is higher than the rates observed in Experiments 1 and 2 and by Cowan (2012). An important difference is that Experiment 3 had community participants who were generally in their 30s, whereas the other studies all used undergraduate participants who were 18-20 years old. However, nearly three participants out of 10 reported incorrectly that an alibi was the evidence that supports a narrative, suggesting that fundamental misunderstandings of the term “alibi" are still common. As in Experiments 1 and 2, participants' understanding of the term “alibi" did not affect their legal judgments.

Limitations. A few limitations may have affected the results. First, participants were given very little information with which to make their judgments. The fictitious newspaper article that presented the case was approximately one letter-sized page and described the case in very general terms. In contrast, a juror would listen to days, weeks, or months of testimony before rendering a judgment of the accused. Naturally, it is not possible to engage participants for studies about legal decision-making for a similar timeframe. Regardless, it is possible that participants' judgments would be less influenced by the independent variables or 
Authoritarianism if they had access to more information. It stands to reason that participants would be more influenced by one piece of information if that was the only information they were given than they would be by the same information if they were provided with other interesting, relevant facts. Thus, the independent variables included in this study should be investigated again using materials more similar to Experiments 1 and 2 to determine whether their effects remain in the context of other information. In general, it might be wise to include more details about the alibi in a study that is investigating alibis, even though no aspects of the alibi itself were manipulated in the current design.

Second, one of the challenges of online research is ensuring that participants take the study seriously and attend to the materials and questionnaires. Unlike with in-lab experimental designs, it is not possible to observe participants completing the task or to attempt to gauge their interest in and attention to the experiment during the debriefing phase. Approximately one fifth (21\%) of participants were excluded from the study because they provided a wrong answer to the manipulation check items about the defendant's race or the city where the crime took place. On average, participants whose data was retained took approximately 15 minutes to complete the task. Some participants who were excluded completed the task much more quickly (i.e., 8-10 minutes), but many of them took approximately 15 minutes as well. This is a relatively high rate of exclusions. In future online studies, it may be beneficial to emphasize the importance of the research and the implications at the outset, as this may lead participants to read the materials and consider their responses carefully. Alternately, participants may attend to the details of the materials more carefully if they are told at the outset that there will be a memory task as part of the study. 
Conclusions. The results of Experiment 3 suggested eight general conclusions:

1. Less favourable legal judgments may be made when the defendant is First Nations, particularly when racially charged terms like "Indian" are used.

2. Compared to those with more positive views, evaluators who hold more negative views about First Nations people are more likely to provide legal decisions that are less favourable for First Nations defendants.

3. Participants' general feelings about White people are unrelated to their legal judgments.

4. White participants rate themselves as less similar to a First Nations defendant than a White defendant, and rate a First Nations defendant as a somewhat worse person than a White defendant. However, Defendant Race did not affect how much they liked the defendant and how they would feel if he moved in next door, was a coworker, or was in a romantic relationship with a family member of the participants. In general, participants had somewhat negative feelings in these cases.

5. Evidence for in-group bias - with participants' nationality determining in-groups and outgroups - was not found.

6. Higher ratings on the Authoritarianism subscale of the ACT were associated with some less favourable ultimate judgments in the case.

7. Religious orientation does not have a strong, consistent relationship with legal judgments.

8. Approximately half of the sample provided an accurate definition for the term "alibi," suggesting that misunderstandings of the term are relatively common. The accuracy of participants' definitions was unrelated to their legal judgments. 


\section{General Discussion}

Broadly, the purpose of this work was to consider the factors that influence or are associated with judgments about alibis. An alibi can be an incredibly powerful piece of evidence for innocence, but there is considerable evidence that honestly offered alibis regularly fail to protect innocent people from wrongful prosecution and conviction (e.g., Burke et al., 2007; Garrett, 2011). The implication here is that an alibi may be a paper shield, giving the impression of assistance to innocent suspects, but offering no real protection. This requires us to ask a number of questions, among them: Why are these alibis being rejected? How are evaluators making the decision whether to accept or reject an alibi? Do evaluators know what an "alibi" is? Under what conditions does an alibi benefit a suspect relative to the absence of an alibi? Are some innocent alibi providers at greater risk because of what their alibi is or their race/ethnicity? Are evaluators' decisions influenced by aspects of their own personalities or belief systems? These are all empirical questions, and psychology and law is well situated to generate the evidence required to provide answers.

The literature on alibi believability is at a nascent stage and the tendency has been-as in most new areas of inquiry - for research to be conducted on a number of sub-topics without an overarching model of how the sub-topics may fit together. Thus, one of the aims of the current work was to begin to integrate some of these disparate research findings to identify the primary determinants of alibi believability judgments and to investigate under which conditions other variables operate. A second primary aim was to introduce some as-yet uninvestigated variables. In the project's initial conception, the moral desirability of the activities described in the alibi was intended to be the variable that this work would add to the research literature, and was included in Experiments 1 and 2. Religiosity, a variable that had not been included in psychology 
and law literature previously, was added in Experiment 3. Results from the three experiments in this work contribute to our understanding of the influence of aspects of the alibi itself, alibi provider characteristics, and evaluator characteristics.

\section{Providing an Alibi Can Be Beneficial or Detrimental for Criminal Suspects}

A tacit assumption in the legal sphere is that offering an alibi will not generally be detrimental to a suspect or defendant. Under Canadian law, an alibi should benefit the suspect/defendant if it is judged as credible; if jurors believe that an alibi is true, they are required to vote not guilty $(R v$ Hibbert). Additionally, in the absence of evidence of deliberate fabrication, a disbelieved alibi should be treated as having no evidentiary value ( $R v$ Hibbert). Deliberate fabrication, however, can be held against the suspect/defendant. Thus, in general, alibis should be either helpful to defendants or neutral. This was the case in Experiment 1. Here, the suspect's statement was rated as more honest, the strength of the evidence against him was rated as marginally weaker, the likelihood of his guilt was rated as lower, and there was a nonsignificant trend for fewer guilty verdicts when he provided an alibi compared to when he could not remember his whereabouts. Some non-significant effects were also found, but there were no examples of alibis leading to less favourable ratings compared to the control.

In contrast, Experiment 2 found that the evidence against the suspect was rated as stronger, the likelihood of his guilt was marginally higher, and there was a marginally greater number of guilty verdicts when the suspect offered an alibi than when he did not. No significant differences were found for the other dependent variables. In this experiment, providing an alibi had no benefit to the suspect, and even potentially damaged his case in the eyes of evaluators.

It is reasonable to ask why two experiments with similar methods generated contradictory results on some of the same dependent measures. As noted in the Discussion of Experiment 2, 
the answer may lie in one small difference in the materials of the two experiments. The case files shown to participants in Experiment 1 included a Supplemental Evidence Report that detailed the evidence that police had uncovered to support the suspect's alibi. The strength of that evidence was manipulated between participants. This document was omitted from the case files in Experiment 2 because that manipulation was not included in the study design. The fact that police ostensibly investigated the alibi in Experiment 1 may have led participants to infer that the police were taking the alibi relatively seriously. Participants may have assumed that if an alibi is obviously false, there would be no need to waste police resources investigating it, so the suspect's alibi in this case must be at least a little bit credible at first glance. In contrast, the Experiment 2 materials made no mention of the investigating officers following up on the suspect's alibi, which may have implied that the alibi did not even achieve sufficient credibility to warrant being investigated. This is similar to Sommers and Douglass' (2007) argument that the reason that an alibi is viewed as less credible in a trial context than an investigative context is because in the latter, participants assumed that if the suspect had gone to trial, the evaluators in the investigation must have determined that the alibi was not credible enough to establish the suspect's innocence. Similarly, in Experiments 1 and 2, the way in which the alibi was presented may have provided evaluators with subtle clues regarding how the alibi is viewed by others and that these clues, in turn, affected the evaluators' judgments. Essentially, these findings could be a case of informational social influence (i.e., individuals using others' behaviour as a cue for how they should behave) operating in the legal system.

\section{Aspects of the Alibi Itself}

Innocent suspects may have been doing nearly anything at the time a crime in which they had no involvement was committed, though Garrett (2011) noted that the majority of the 
individuals exonerated by the Innocence Project who offered alibis at trial indicated that they were home alone or with family. Regardless, the content of the alibi is one aspect that is likely to vary between suspects. This variability also influences the evidence that might be available to corroborate the alibi. An individual who was at home alone would have a much more difficult time corroborating an alibi than an individual who was celebrating his/her birthday with many friends, at a restaurant that had CCTV cameras, and who paid the bill with a credit card that required a signature (see Burke et al., 2007). Indeed, some alibis may simply be inherently noncorroborable (Olson \& Charman, 2012). Results indicate that aspects of the alibi itself - its content and the strength of the evidence that supports it - may affect evaluators' judgments.

Alibi moral desirability. Two competing theories predicted different patterns of effects for alibi moral desirability. Briefly, the first theory was that evaluators would form an implicit personality theory about the alibi provider based on the content of his/her alibi, and that their judgments would be consistent with these implicit personality theories (Allison, Mathews, \& Michael, 2012). In particular, evaluators would infer that an individual offering a morally undesirable alibi was a bad person in general and would be less likely to believe the alibi than a neutral alibi, and the opposite for a morally desirable alibi. Basic social psychology research supports this hypothesis. There is a general tendency for humans to make dispositional attributions about the behaviour of others and neglect the potential effects of the situation (i.e., Fundamental Attribution Error; e.g., Jones \& Harris, 1967) and to assume that if someone has one positive or negative characteristic, they must have other consistent characteristics (e.g., Corsini, 1999; Greenwald \& Gillmore, 1997; Nisbett \& Wilson, 1977). These general attributions affect legal decision-making, in particular with respect to the physical and social attractiveness of the defendant (e.g., Bull \& Rumsey, 1988; Downs \& Lyons, 1991; Efran, 1974; 
Landy \& Aronson, 1969; Levelthal \& Krate, 1977; Solomon \& Schopler, 1978; Stewart, 1980) and inferences related to defendant facial characteristics (e.g., Berry \& Zebrowitz-McArthur, 1988; Zebrowitz \& McDonald, 1991).

Alternately, Allison, Mathews, and Michael (2012) suggested that an alibi that involves admitting to a misdeed could be taken as evidence that the suspect is being honest. This hypothesis is supported by literature that suggests that the stories told by liars are more likely to present the liar in a positive than a negative light (Aune \& Waters, 1994), that guilt may be rated as lower when the target admits guilt to a lesser offense than when the target merely denies involvement in an offense (Sternglanz, 2004), and some evidence that the "salaciousness" or moral desirability of the alibi activities affect legal judgments (Allison et al., 2012; Cowan 2012, but see also Allison et al., 2013 and Jung et al., 2013 for non-significant results).

Experiments 1 and 2 did not provide clear, consistent support for either theory. In Experiment 1, alibi moral desirability had complex, interactive effects, and only affected some of the dependent measures. In particular, alibi moral desirability tended to have significant effects only when there was no physical evidence to corroborate the alibi. In some cases, alibi moral desirability affected results at Iowa State University, but not at Ryerson University. Also, when there were effects at both locations, the pattern of effects was not consistent. The results at Iowa State constituted weak support for both theories. The morally desirable and morally undesirable alibis were both preferable to the neutral alibi, in terms of likelihood of guilt, verdict choices, and willingness to sign the petition supporting the suspect. Essentially, the result from Iowa State suggest that offering a morally undesirable alibi might be interpreted as a sign of honesty, and offering a morally desirable alibi might mark the suspect as being a generally good person. In contrast, the results at Ryerson showed evidence of participants rejecting morally undesirable 
alibis (i.e., negative implicit personality theory) and treating morally desirable and neutral alibis the same.

The comparison between each alibi narrative and the control condition in Experiment 1 offers some support to the first theory. The morally desirable alibi was significantly different than the control condition more consistently than were the morally undesirable or neutral alibis. Unfortunately, it was not possible to determine whether this pattern was consistent across the two samples, as there were too few participants in the control condition at either location to generate the power required for statistical tests.

In Experiment 2, which was only conducted at Ryerson, the general pattern of results was that morally desirable activities were seen as suspicious (i.e., morally desirable alibis led to ratings less favourable for the suspect than the control condition), but morally undesirable alibis were not seen as indicating honesty (i.e., morally undesirable was not significantly different than control). This is not consistent with the first theory, that participants' judgments are consistent with their implicit personality theories, nor is it consistent with the second theory, as morally undesirable alibi activities did not exert a protective effect. It is also worth noting that some of the effects of alibi moral desirability were only found when the suspect was First Nations and not when the suspect was White. Interestingly, the overall pattern of effects is not the same as what was observed at Ryerson in Experiment 1. In the first, morally undesirable alibis led to the least favourable ratings for the suspect, whereas in the second, morally desirable alibis were the least beneficial to the suspect.

The fact that there were many interactive effects is also evidence against both of the proffered theories. Both theories predicted that alibi moral desirability should have clear, unique effects on judgments. Instead, the effects of alibi moral desirability appear to be contingent on 
other factors (e.g., lack of corroborative evidence, possibly only or primarily for suspects who are not White). It seems that either the effects of alibi moral desirability are more complex than current theories explain, or that the results of Experiments 1 and 2 are spurious. Additional research is required to determine under what conditions particular patterns of results may be replicable. For example, are evaluators' reactions contingent on other specific aspects of the case or on individual difference or ideological belief factors like Social Dominance Orientation?

Interestingly, alibi moral desirability did affect participants' non-legal judgments. Across both experiments, participants' responses were the most favourable in terms of liking the suspect and thinking he was a better - or less bad - person when he offered a morally desirable alibi, and were the least favourable when he offered a morally undesirable alibi. The same pattern was observed for participants' ratings of how similar they believe they are to the suspect. This is evidence that participants formed general implicit personality theories about the suspect, but, as discussed previously, these theories did not have a clear, consistent effect on legal decisions.

In interpreting these results, it is important to consider the success of the alibi moral desirability manipulation. The results of the manipulation check items suggested that the Morally Desirable and Neutral conditions were effective as, in both experiments, the two conditions were significantly different from each other and only ratings for the Morally Undesirable condition were significantly different from the midpoint of the scale. Additionally, previous research by Cowan (2012) showed that having an affair with a friend's partner was evaluated very negatively by the majority of participants. Whether the alibi in the Morally Desirable condition was perceived as intended is less clear. Ratings regarding the behaviour showed the desired results, though the difference between Morally Desirable and Neutral was smaller than the difference between Morally Undesirable and Neutral. However, participants' ratings about an actor who 
had engaged in the behaviour were often not different from Neutral. This may be due to the manipulation being weaker than would be ideal, for example if participants interpreted the activity of helping a friend move to mean socializing while unpacking a few boxes. Alternately, it is possible that the items assessing the manipulation were unable to capture the difference. In the hypothesis-testing analyses, the Morally Desirable and Neutral conditions did not perform identically.

One additional point regarding alibi moral desirability is necessary before moving on to other topics. If the neutral alibi is the least beneficial option for suspects, as it was at Iowa State in Experiment 1, this is a particularly troubling result for innocent suspects. As noted earlier, the majority of alibi defenses raised by individuals later exonerated by the Innocence Project were arguably morally neutral (i.e., at home alone or with family). These are already the alibis that would be most difficult for innocent suspects to recall, due to being low in salience, emotional meaning and valence, and obvious motivation to encode details of the event (Read \& Connolly, 2007). These suspects, then, would be at an additional disadvantage if reporting neutral behaviour in an alibi is considered more suspicious than other alibi narratives. However, it must be said again that this was only the pattern of results in one of three samples. In the other two, neutral was not the most disadvantageous alibi.

The best defense is strong physical evidence. Experiment 1 also manipulated the strength of the physical evidence that supported the suspect's alibi, based on Olson and Wells' (2004) taxonomy. In general, results supported the taxonomy, with strong evidence being helpful to suspects, relative to having no evidence. For some dependent measures, weak evidence was also preferable to having no evidence. However, no differences were observed between strong and weak evidence categories, suggesting that evidence that is relatively difficult to fabricate is 
not significantly more beneficial to suspects than evidence that is easier to fabricate.

Interestingly, alibi evidence strength affected responses more consistently in the Ryerson sample than in the Iowa State sample, though it is not clear why. For several dependent variables, alibi moral desirability only affected the results when there was no physical evidence available. This suggests that the evidence that supports an alibi is a primary determinant of evaluator judgments, and that other variables are only considered when information about corroboration is unavailable.

The discovery that the strength of the evidence that supports an alibi is the primary determinant of judgments about it is both encouraging and discouraging from the perspective of wrongful convictions. It is encouraging because it suggests that participants are more influenced by potentially useful, valid information than they are by stereotypes and instinctive assumptions. It seems logical that an innocent alibi provider would be more able to provide strong evidence to support his or her claim than would an individual who is attempting to fabricate an alibi, though this remains an empirical question. Faking or altering CCTV footage would be no easy task for the average person. However, few criminal defendants in Canada and the United States corroborate their alibis with any physical evidence (e.g., Burke \& Turtle, 2003) - the more persuasive form of corroborative evidence - and the majority of person evidence for innocent alibi providers appears to come from individuals who have an obvious motive to lie for the defendant (Garrett, 2011). Although the provision of strong corroborative evidence may be diagnostic in terms of distinguishing between True and Fabricated alibis (i.e., True is significantly more likely to have strong evidence than Fabricated), many innocent defendants may be unable to meet the expectations that evaluators hold regarding the quality, amount, and type of evidence that should be available to support a truthful alibi. 


\section{Alibi Provider Characteristics - Suspect/Defendant Race}

It is also likely that evaluators are influenced by the characteristics of the alibi provider him- or herself. In particular, there is compelling evidence from criminal cases and from empirical research that members of minority groups are at a disadvantage in the legal system. The incarceration rate for Canadian Indigenous people in Canada is approximately ten times the incarceration rate of non-Indigenous people, and this disparity has been linked directly to discrimination and prejudice against Indigenous people (Office of the Correctional Investigator, 2013). Additionally, 60-70\% of exonerated people (The Innocence Project, n.d.d.; National Registry of Exonerations, 2013) are members of visible minority groups. There is also substantial evidence from lab-based research that White participants treat Black defendants more harshly than White defendants in mock trial paradigms, at least when race is not salient (e.g., Mitchell et al., 2005; Sommers \& Ellsworth, 2001), and that Native Canadian defendants are treated more harshly than English or French Canadian defendants (Pfeifer \& Olgoff, 2003). With respect to alibis in particular, however, the only three studies to date showed that the Black suspect was treated more leniently than the White suspect, presumably out of participants' desire to avoid appearing racist (Cowan et al., 2015; Marion et al., 2011; Sargent \& Bradfield, 2004).

Experiments 2 and 3 manipulated whether the suspect/defendant what White or First Nations to investigate whether this factor affected participants' responses. In Experiment 2, suspect race had few significant effects, though effects for alibi moral desirability on ratings of statement honesty and alibi accuracy were often found for only the First Nations suspect. This is consistent with Sargent and Bradfield's (2004) finding that evaluators may process case information differently, and attend to more information, when the defendant is Black compared to when he is White. Additionally, in Experiment 2, participants were more willing to sign a 
petition supporting the First Nations suspect than the White suspect. Finally, participants' general feelings about White and about First Nations people were unrelated to their legal judgments.

In contrast, Experiment 3 showed a fairly consistent pattern of less favourable ratings for the First Nations defendant than the White defendant, though there was often an interaction with crime location such that the race effects were only significant when the crime was set in Seattle. As noted in the Discussion for Experiment 3, this may be due to the use of different terms for the Indigenous defendant when the crime was set in Seattle ("American Indian") and Vancouver ("First Nations") activating different stereotypes in the minds of evaluators. Additionally, a linear relationship was found between participants' general feelings about First Nations people and their legal judgments, with more positive ratings being associated with legal judgments that are more favourable for the defendant. This pattern was not found for participants' general feelings about White people. To sum up, the results of Experiment 2 generally suggest no consistent pattern of suspect race exerting a significant unique effect on legal judgments, whereas the results of Experiment 3 indicate that First Nations defendants are at a significant disadvantage.

The difference in the pattern of results between the two studies is likely attributable to differences in the materials that led race to be much more salient in Experiment 2 than it was in Experiment 3. In Experiment 2, participants read a fictitious police file that included a Suspect Information Sheet with a photograph of the suspect and a written record that he was either White or First Nations. In contrast, the materials from Experiment 3 were relatively brief and did not include a photo of any kind. Previous research indicated that race salience is an important factor in how race affects participants' judgments. Sommers and Ellsworth (2001) argued that when 
race is salient, social norms to avoid appearing racist are activated. This is consistent with what Marion et al. (2011) and Cowan et al. (2015) found with respect to alibis, using materials that were very similar to the materials used in Experiment 2. The much subtler manipulation in Experiment 3 may not have activated these social norms, meaning that participants were less likely to self-censor while responding to the questionnaire items.

\section{Alibi Evaluator Characteristics}

The characteristics of the individuals evaluating an alibi are also relevant to the discussion of alibi credibility judgments. Alibi evaluators bring their own stereotypes, schemas, personalities, and experiences into the decision-making process. As Olson and Wells (2012) discovered, one experience that affects evaluators' judgments about alibis is the act of generating their own alibi. Particularly relevant for this work are the influences of evaluator race/ethnicity, in-group bias, Authoritarianism, religious orientation, and participants' understanding of the term "alibi."

Evaluator race. One of the key findings from Experiment 1 was that the results differed between the samples collected at Iowa State and at Ryerson. One post-hoc explanation for this was the difference race/ethnicity of the samples; the sample from Iowa State was almost exclusively White, whereas the sample from Ryerson was highly diverse. Some support was found for this hypothesis. White participants at Iowa State provided ratings that were more favourable for the suspect than did People of Colour (POC) at Ryerson, but White participants at Ryerson were not significantly different from either group. It is possible that there were too few White participants at Ryerson to detect significant differences between their responses and the responses of either of the other two groups. The results would have been more compelling if White participants at Ryerson provided responses that were significantly different than POC at 
Ryerson, but not significantly different from White participants at Iowa State. Experiment 2, which was conducted only at Ryerson, did not show any differences in responses between White and POC participants, even though nearly half of participants were White. The sample of Experiment 3 was nearly entirely White, so analyses based on participant race/ethnicity were not computed. It seems as though participant race/ethnicity may influence legal judgments, but that other contextual factors are also important in determining when and if these effects emerge.

In-group bias. A second post-hoc explanation for the location effects in Experiment 1 was in-group bias - the tendency for individuals to show favouritism towards individuals who are also members of a group with which the individual feels a psychological affiliation over individuals who are not members of the group. In Experiment 1, the crime was always set in Seattle, WA, meaning that participants at Iowa State would be more likely to view the suspect as an in-group member (i.e., another American) than participants at Ryerson. Indirect evidence was found for this explanation in Experiment 1, as participants at Iowa State rated themselves as more similar - or, more accurately, less dissimilar - to the suspect than participants at Ryerson, though no differences were found between the samples for participants' ratings of how much they liked the suspect, and the marginal effect may have been due to the greater number of male participants at Iowa State than at Ryerson.

Experiment 2 assessed in-group bias by manipulating whether the crime took place in Seattle (i.e., American suspect) or Vancouver (i.e., Canadian suspect), with an all-Canadian sample. However, this manipulation was added partway through data collection and was quite subtle until participants viewed the petition website at the end of the experimental session. It is likely that participants did not attend to this information while reading the case file and only noticed the location when they saw the recipient of the petition, either Legal Aid Washington or 
Legal Aid British Columbia. The only significant difference for crime location was that participants were more willing to sign the petition when the crime was set in Vancouver than when it was set in Seattle, consistent with what would be expected if in-group bias was operating. Additionally, participants' ratings of how similar they are to the suspect, how much they like the suspect, and whether they thought that the suspect was a good or bad person-all collected before the petition-were unaffected by whether the crime was set in Seattle or Vancouver.

Experiment 3 was designed specifically to assess the role of in-group bias, combining the participant nationality manipulation from Experiment 1 and a stronger version of the crime location manipulation from Experiment 2. Interactions between these two factors in which participants from Canada and the United States were each more lenient towards defendants of the same nationality would constitute evidence for in-group bias. However, this interaction for legal judgments was only found with verdict choice; Canadian participants provided more guilty verdicts for the American defendant, though the effect was not statistically significant, American participants appear to have provided more guilty verdicts to the Canadian defendant, though the number of guilty verdicts was too low to allow for inferential statistical testing. The expected interaction was not found either for non-legal judgments (e.g., similarity, liking).

Thus, across the three experiments, the evidence for in-group bias is weak. The strongest evidence for in-group bias comes from Experiment 1, but this is the experiment that did not include specific manipulations to identify in-group bias. The general conclusion, therefore, is that in-group bias based on nationality does not exert a strong influence on legal judgments.

Authoritarianism. Authoritarianism is the one personality/social orientation variable that has been consistently related to legal judgments in the literature. In particular, higher 
endorsement of authoritarian views is associated with a greater number of guilty verdicts and convictions (Devine, Clayton, Dunford, Seying, \& Price, 2001; Narby, Cutler, \& Moran, 1993), longer recommended sentences (Devine et al., 2001), support for capital punishment (McKee \& Feather, 2008) and more skepticism of alibis (Cowan, 2012; Culhane, 2005). Therefore, it was hypothesized that individuals who endorsed more authoritarian views would provide legal judgments that were generally unfavourable for the suspect/defendant, relative to those who do not strongly endorse these views. Some evidence was found to support this assertion.

In Experiment 1, higher total and subscale scores on the Authoritarianism-ConservatismTraditionalism (ACT) scale (Duckitt et al., 2010) were fairly consistently related to ratings that were less favourable for the suspect. As well, the relationship between scores on the Authoritarianism subscale and legal judgments was mediated by participants' perceptions of the morality of the alibi activities; participants who scored higher on Authoritarianism were more likely to view the alibi activities negatively, and these negative views were related to lower ratings of statement honesty and alibi accuracy, higher ratings of the strength of the evidence against the suspect and likelihood of guilt, and more confidence in alibi accuracy judgments. However, no clear, consistent pattern of effects for Authoritarianism and no mediation effects were found in Experiment 2, and only the Authoritarianism subscale showed significant relationships with only some of the outcome measures in Experiment 3. One might argue that the lack of significant findings indicates that participants were not attending to the questionnaire task. This seems unlikely, however, as all significant findings were in the predicted direction. If participants were generally responding randomly, the spurious significant effects should be distributed across both directions.

It seems as though scores on the Authoritarianism subscale are particularly relevant to 
legal judgments, as significant mediation effects in Experiment 1 and significant linear relationships in Experiment 3 were particularly found for this subscale. In fact, this is consistent with Duckitt et al.'s (2010) results. In their work to establish the discriminant validity of the subscales of the ACT, they found - as predicted - that only the Authoritarianism subscale was significantly associated with longer sentence length recommendations. This is entirely logical, as this subscale stemmed from Altemeyer's (1981) original conceptualization of Authoritarian Aggression. The subscale measures an individual's willingness to exact harsh punishments on those they feel are deserving, particularly for violating traditional social norms. Items on the scale involve the need to take strong action to deal with criminals, the need for strict laws and punishment for "troublemakers" and "perverts." In the legal sphere, issues of responsibility and punishment are generally at the forefront of evaluators' minds. From a theoretical perspective, these results support Duckitt and colleagues' (e.g. Duckitt, 1989; Duckitt et al., 2010; Duckitt \& Fisher, 2003; Jugert \& Duckitt, 2009) contention that Authoritarianism is better conceptualized as a set of related multidimensional constructs regarding social values, than as a unidimensional personality construct, as Altemeyer (1981) argued.

Religiosity. It was also hypothesized in Experiment 3 that religious orientation might be related to legal judgments. In particular, individuals who had an Extrinsic religious orientation were expected to see themselves as superior to the defendant and worthy of judging him, leading to harsher ratings. Competing theories were offered for the effect of Intrinsic religious orientation, and no specific hypotheses were provided for Quest orientation. However, no consistent significant relationships were observed between participants' responses on any of the subscales of the Revised Religious Life Inventory (RLI-R; Hills et al., 2005) and their legal judgments, and no differences were observed between participants who provided ratings for the 
majority of the items (i.e., High-Responders) and participants who chose the "Not Applicable" option for the majority of the items (i.e., Low-Responders). Based on these findings, it seems as though participants' feelings about and general approach to religion are unrelated to their judgments in a legal context.

\section{Misunderstandings About the Term "Alibi" Abound}

A final goal of the current project was to conduct a systematic investigation of adults' understanding of the term "alibi." An incidental finding by Cowan (2012) was that that many participants misunderstood what was meant by this term. Consequently, in Experiments 1-3, participants were asked to define "alibi" in their own words. Across the three samples, a minority of participants correctly indicated that an alibi is a narrative - often supported by evidence - that a criminal suspect was elsewhere at the time of a criminal event and, consequently, could not possibly be the perpetrator. In Experiments 1 and 2, the most common response was that an alibi was the evidence that supported the narrative, rather than being the narrative itself. In Experiment 3, this was the second most common response; the most common response was a correct definition, though fewer than 50\% of participants were correct. Interestingly, accurate responses were more frequently provided when participants were community members who were generally in their 30s (Experiment 3) than when participants were undergraduate students (Experiments 1 and 2). Adults in Experiment 3 may have been incidentally educated regarding legal issues in the course of their interactions with the legal system, watching television, reading newspapers, or the like. However, qualitative research is not undertaken with the intention of generalizing findings beyond the sample, so it would be inappropriate to make strong claims regarding whether this particular pattern is likely to emerge within other samples.

Although no differences were observed between participants who provided correct 
definitions and participants who erroneously prioritized alibi evidence in terms of direct legal judgments, it is possible that misunderstandings about what an alibi is have other, potentially more subtle effects. One obvious implication is the need to have clear instructions to jurors regarding alibi issues. In Canada, after the Crown and Defense have presented their final arguments, the judge provides a charge to the jury in which s/he instructs them about legal matters that are relevant to the decision that the jurors must make. This generally includes an explanation of the charges and the legal requirements for verdict options. Whenever relevant, this will generally include instructions specific to alibis. Based on the current research, it is recommended that these instructions include - and preferably begin with - a statement from the judge defining what an alibi is. It may also be beneficial to explain what an alibi is before that part of the defense case is presented during direct examination.

\section{Implications for Research and Practice}

The differences in results between the Ryerson and Iowa State samples in Experiment 1 lead to an important question for researchers: how far can research results be generalized? Psychology is often concerned with identifying broad trends that apply to the majority of individuals. The results of Experiment 1 suggest that, with regard to alibi judgments at least, care should be taken in generalizing research results to locations where there may be important social or cultural differences. The majority of English-language research in the field of psychology and law is conducted in the United States, and there is a possibility that the results may not translate directly to Canada. However, few differences were observed between the Canadian and American samples in Experiment 3, suggesting that generalizing between the two countries is appropriate, at least when the samples are collected from across both nations. Generalizability of findings remains an empirical question, one that can only be answered by a robust literature 
comparing results across different locations, both between and within countries.

There is a growing empirical literature investigating the benefits and drawbacks of sampling using Amazon's Mechanical Turk (mTurk). In general, mTurk samples are relatively diverse and tend to be older and more representative of the country in which they live than the majority of undergraduate samples (e.g., Berinsky, Huber, \& Lenz, 2012; Bukrmeister, Kwang, \& Gosling, 2011; Paolacci \& Chandler, 2014). In the current work, the mTurk sample was notably older than the other two samples, but was less ethnically diverse than the samples from Ryerson University. In general, research findings generated with undergraduate samples have been replicated with mTurk samples (e.g., Berinsky et al., 2012). Although there are challenges - in particular with the relatively high rate of exclusions in Experiment 3 related to failed manipulation check items - mTurk appears to be a valuable tool for increasing the diversity of samples.

In terms of implications for the legal system, the results of the current studies suggest that decisions about alibis are complex and may be affected by the qualities of the alibi itself as well as the individual evaluating the alibi. The challenge is that some of the variables that may affect or be related to judgments are likely to introduce bias, in particular suspect/defendant race. It is important, then, to develop processes that mitigate this potential bias. One option could be by taking steps to inform evaluators about the influence of race on judgments about alibis. Canadian and American legal systems acknowledge the potential for racial bias, but this does not appear to be explicitly considered with respect to alibis. The increase in race salience could mitigate the bias, as Sommers and Ellsworth (2001) found.

\section{Avenues for Future Research}

Although the current work provides valuable insight into the influence of factors related 
to the alibi itself, the alibi provider, and the alibi evaluator, there remain many unanswered questions regarding how alibi believability judgments are made. In terms of alibi content, there are a number of other variables that merit investigation. The level of harm done to a victim of crime affects attributions of blame (e.g., Austin, Walster, \& Utne, 1976; Wissler, Even, Hart, Morry, \& Saks, 1997) and sentence-length decisions (e.g., Kerr \& Kurtz, 1977). For example, participants assigned harsher penalties to a convicted rapist when the rape resulted in pregnancy compared to when it did not (Scroggs, 1976). As well, longer sentences were recommended for a convicted murderer when victim impact statements from family members described higher levels of psychological and financial harm (Myers, Lynn, \& Arbuthnot, 2002). It is of interest whether this same pattern applies to the circumstances of an alibi. For example, it is possible to have an alibi that involves the suspect committing a minor theft and manipulating the consequences to the store clerk for not noticing or preventing the theft as it was occurring. The clerk could be fired, given a formal disciplinary notice, or not be punished at all. Greater harm done to others in the alibi narrative would be expected to result in harsher judgments from participants in terms of sentence length. It is unclear, however, how this may affect alibi believability judgments; as with moral desirability, admitting a misdeed with a more negative outcome could be interpreted as indicating honesty or as a reflection of the suspect's generally poor character.

The deception detection literature is also fertile ground for identifying alibi content variables. Research has identified cues that are typically used to discriminate between truthful and deceptive statements, including: stuttering, use of clichés, plausibility of the narrative, pauses, consistency of details, statement coherence (e.g., Akehurst, Köhnken, Vrij \& Bull, 1996; Global Deception Research Team, 2006). These cues are not necessarily actually indicative of deception. Rather, these are the cues that individuals use. Any of these could relatively easily be 
manipulated within an alibi statement to determine if they are relevant in this context.

A final content-related manipulation is the congruence between participants' event schemas and the details of the event described in an alibi. Individuals pay more attention to and are more likely to encode schema-consistent details (Tuckey \& Brewer, 2003) and are more likely to retrieve these details from long-term memory (e.g., Brewer \& Nakamura, 1984; Taylor \& Crocker, 1981). Individuals may also recall incorrect schema-consistent details (e.g., Greenberg, Westcott, \& Bailey, 1998). Additionally, schemas affect judgments in many domains, including: determinations of the cause of personal success (Bryan, Dweck, Ross, Kay, \& Mislavsky, 2009), consumer decisions (Nakayachi \& Kojima, 1988), clinical decisions among nurses (Tabak, Bar-tal, \& Cohen-Mansfield, 1996). Alibis that involve a number of schemainconsistent details may be more likely to seem suspicious, given their unusual content. Alternately, alibis that are too consistent with schemas may not be believed due to lacking unique details that personalize the account.

Although the results of Experiment 1 were generally consistent with Olson and Wells' (2004) taxonomy, their results were not perfectly replicated. Significant differences were observed consistently between the strong physical evidence and no physical evidence conditions, and sometimes between the weak physical evidence and no physical evidence conditions. However, the strong and weak conditions were not significantly different from each other. Based on these results, it is not clear that strong and weak physical evidence are distinct categories that affect evaluator judgments differently. Consequently, a replication of Olson and Wells' (2004) study, using their materials if possible, is advisable. Although their taxonomy is entirely logical, it was established in only one study, and supporting evidence has only included a subset of their person variable conditions (e.g., Culhane \& Hosch, 2004; Hosch, Culhane, \& Howley, 2005; 
Sargent \& Bradfield, 2004). The current work is apparently the first attempt to replicate Olson and Wells' (2004) findings for the strength of physical evidence.

\section{General Conclusions}

The current studies contributed to the literature on alibi believability by considering factors related to the alibi itself, the alibi provider, and the alibi evaluator. The question of how individuals decide whether to believe an alibi or not is a relatively new one within psychology and law, and there is much work still to be done to provide a satisfactory answer. The results of the current work indicated that providing an alibi - relative to not providing one - may be beneficial (Experiment 1) or detrimental (Experiment 2) for the suspect, depending on the content of the alibi, the strength of the physical evidence that supports the alibi, and the race/ethnicity of the suspect. As well, the content of the alibi itself - specifically, the moral desirability of the activities described in the alibi - may influence these judgments, but it is not entirely clear precisely what effect this has, and under what conditions the effect emerges. In particular, alibi moral desirability may be influential particularly or only when there is no physical evidence to corroborate the alibi and when the suspect/defendant is First Nations (or not White). And it seems as though the strength of the physical evidence supporting the alibi remains the primary determinant of credibility judgments. Given the multitude of factors that appear to influence judgments about alibis, it seems as though alibis likely provide adequate protection to some innocent suspects (e.g., with strong corroborative evidence, a particular narrative, and suspects who are White) and insufficient protection to others (e.g., corroboration from family members, members of visible minority groups).

The current work, principally Experiment 3, has also added to the literature showing that suspects or defendants who are members of visible minority groups are at a disadvantage in the 
legal system. At the very least, suspect race affected participants' judgments, which is contrary to the principle of justice that everyone is equal under the law. Experiment 3 showed that less favourable responses were provided when the defendant was First Nations than when he was White, and that participants who had generally more negative feelings about Indigenous people were relatively more likely to provide responses that were disadvantageous for the First Nations defendant. Interestingly, participants' general feelings about White people were unrelated to their legal judgments.

As well, there was evidence that at least some characteristics of the alibi evaluator himor herself influenced responses. Higher scores on the Authoritarianism subscale of the ACT (Duckitt et al., 2010) were consistently associated with judgments that were less favourable for the suspect/defendant, across the three experiments. Weak evidence was also found that the race/ethnicity of evaluators may influence their judgments, but the pattern of effects was not consistently found across studies. Some indirect evidence was found indicating that in-group bias affects judgments about alibis, but the results of Experiment 3, which was specifically designed to test this hypothesis, did not show in-group bias in participants' responses. As well, participants' religious orientation was unrelated to any of their legal judgments.

Finally, results of the three studies indicated that a large proportion of participants did not show an intuitive understanding of what is meant by the term "alibi." Many participants erroneously reported that an alibi is the evidence supporting a narrative about a suspect's whereabouts, when a more accurate definition is that an alibi is the narrative itself. It seems prudent, then, to provide clear instructions to jurors regarding definitions and uses of alibis to assist them in their role as triers of fact.

Taken together, the results presented here make a significant contribution to the alibi 
literature. The majority of research to date has emphasized considering one or two variables. This has led to an understanding of alibi believability judgments that is likely oversimplified, particularly given the complex interactive effects observed throughout the current work. Alibi credibility assessment appears to be a complex process. This work and the work of others provides an important basis for creating a comprehensive model to explain how these judgments are made. 


\section{Appendices}

\section{Appendix A: Recruitment Information for Experiment 1}

\section{Ryerson University}

\section{Study Name:}

Study 1: Criminal Investigation 1

Brief Abstract: In this study, we are exploring how people determine the guilt or innocence of a suspect. You will be given information about the initial stages of a police investigation and be asked to answer questions about your impressions about the case. This Social Psychology study involves one 60 minute visit to the lab.

\section{Detailed Description:}

The study will take place at the South Bond Building (105 Bond), 2nd Floor. You can take the stairs or the elevator to the second floor and have a seat in the lobby. Someone will meet you there. If need be, the lab phone extension is $\times 2190$. Approximately $200-250$ participants will be recruited. All participants will be students at Ryerson University currently enrolled in the Introductory Psychology course PSY102 or PSY202. The study will consist of one 60 minute session which may be completed in a group setting, although all data collection is individual. You will be asked to read a short summary of a hypothetical criminal case and then provide feedback about your impressions of the case and the individual involved. You will then be asked to complete a questionnaire designed to measure beliefs and attitudes relevant to the legal context. While no risks are anticipated, it is possible that you may feel slight discomfort in answering some questions relating to attitudes, beliefs, or personal opinions. However, all measures used in this research have been used in previous research and are not believed to cover any particularly controversial topics. You are free not to answer any and all questions you feel uncomfortable answering. By participating in this study, you may benefit from a greater understanding of how research is conducted in the field of Social Psychology. 
Iowa State University

\section{STUDY POSTING FORM}

Ann Schmidt MUST receive a copy of this form before you send an activation request.

PRINCIPAL INVESTIGATOR (Faculty Supervisor): Dr. Christian A. Meissner

RESEARCHERS: Sara Cowan

STUDY NAME \& NUMBER: Criminal Investigation 1

\section{BRIEF ABSTRACT:}

In this social psychology study, you will be asked to read about a criminal case and provide your impressions. The study includes one 60-minute session.

\section{STUDY DESCRIPTION (Must be exactly as approved by IRB):}

The following study includes one session of up to 60 minutes. The study and materials will be presented in English. The study investigates how individuals form impressions about the guilt or innocence of a criminal suspect. You will be asked to read about a criminal case, provide your impressions about the case and suspect, and fill out personality measures.

ELIGIBILITY REQUIREMENTS: Students must be 18 years of age or over and juryeligible (US citizen, no felony convictions, proficient in English, etc.).

DURATION (Minimum 30min.):1 sessions of 60 minutes

CREDITS: 2

PREPARATION: None

IRB APPROVAL CODE:

IRB APPROVAL EXPIRATION:

IS THIS AN ONLINE STUDY? NO

ATTENTION RESEARCHER:

THE STUDY DESCRIPTION POSTED ON SONA MUST BE IDENTICAL TO THAT APPROVED BY IRB. IF YOU NEED TO MODIFY THE DESCRIPTION OF A STUDY, YOU MUST PROVIDE ANN WITH THE NEW IRB-APPROVED DESCRIPTION. 
$12 / 3 / 06$

\begin{tabular}{|l|l|}
\hline Field & Explanation \\
\hline Study Name & Criminal Investigation 1 \\
\hline Brief Abstract & $\begin{array}{l}\text { In this social psychology study, you will be asked to read about } \\
\text { a criminal case and provide your impressions. The study } \\
\text { includes one 60-minute session. }\end{array}$ \\
\hline Detailed Description & $\begin{array}{l}\text { The following study includes one session of up to } 60 \text { minutes. } \\
\text { The study and materials will be presented in English. The study } \\
\text { investigates how individuals form impressions about the guilt } \\
\text { or innocence of a criminal suspect. You will be asked to read } \\
\text { about a criminal case, provide your impressions about the case } \\
\text { and suspect, and fill out personality measures. }\end{array}$ \\
\hline Eligibility Requirements & $\begin{array}{l}\text { Participants must be adults at least 18 years of age } \\
\text { and jury-eligible (US citizen, no felony convictions, } \\
\text { proficient in English, etc.). }\end{array}$ \\
\hline Duration & 60 minutes \\
\hline Preparation & None \\
\hline IRB Approval Expiration Date & \multicolumn{2}{|c|}{$\begin{array}{l}\text { Copyright @ 2005 Sona Systems, Ltd., All Rights Reserved } \\
\text { This page was extracted from the experiment management system documentation (v2.63), further was modified with } \\
\text { permission of Sona Systems to meet lowa State Department of Psychology's specific program needs }\end{array}$}
\end{tabular}




\section{Appendix B: Consent Forms for Experiment 1}

RYERSON

UNIVERSITY

\author{
Ryerson University \\ Consent Agreement \\ Criminal Investigation 1
}

You are being asked to participate in a research study. Before you give your consent, it is important that you read the following information and ask as many questions as necessary to be sure you understand what you will be asked to do.

Investigator:

\author{
Sara Cowan, MA \\ $\mathrm{PhD}$ Candidate \\ Department of Psychology \\ Ryerson University
}

\author{
Supervisor: Dr. Tara Burke \\ Associate Professor \\ Department of Psychology \\ Ryerson University
}

Purpose of the Study: The purpose of this study is to examine how individuals determine the guilt or innocence of a suspect in a crime scenario. In order to study this issue, 200-250 students will be asked to participate in this research. All Ryerson students enrolled in Psy102 or Psy202 are eligible to participate.

Description of the Study: If you decide to participate in this research, you will be asked to do the following: You will read some information about a hypothetical criminal case, read a transcript of a police interview with a suspect, and answer some questions about your thoughts about the case. This study will take place in the Psychology Research and Training Centre at 105 Bond Street. It will take approximately 60 minutes to complete.

What is Experimental in this Study: None of the procedures or questionnaires used in this study are experimental in nature, in the sense that they have all been used by other researchers and found to be useful procedures and questionnaires. From a technical or procedural point of view, part of this study is considered "experimental," because by following the procedure described above, the study examines the impact of one variable (called the "independent variable") on another variable ("called the dependent variable"). You will be given more information about the independent and dependent variables in this study at the end of today's session.

Risks or Discomforts: Occasionally people feel uncomfortable when answering questions that ask about attitudes toward controversial social issues (e.g., affirmative action). While we do not anticipate that any of the questions you will be responding to are controversial, if any aspect of this study makes you feel uncomfortable, you may choose not to answer certain questions, or to withdraw from the study at any time without penalty.

Benefits of the Study: We anticipate that you will benefit from this study by learning about how psychology research is conducted. When the session is over, we will describe the purpose and hypotheses of the study to you in more detail. Also, once we have analysed the data (toward the end of the term), you are welcome to contact the researchers by e-mail to view the results. 
Confidentiality: Your responses in this research will be confidential; your name may be linked to your responses. As such, your responses are not anonymous. Any materials that include your name - including this consent form - will be stored confidentially for a minimum of 5 years. Physical materials will be stored in a locking filing cabinet in a locked room, and computer files will be stored on password-protected computers. Only the investigator and her faculty supervisor will have access to this information. Wherever possible, questionnaires will be identified only by a participant number assigned to you by the researchers. This number cannot later be used to identify you and is unrelated to your Sona ID. This consent form (if you decide to participate) will be filed separately from any data collected in the experiment. The online questionnaire is hosted by Qualtrics, a web survey company located in the USA and as such, is subject to U.S. laws; in particular, the US Patriot Act, which allows authorities access to the records of internet service providers. This survey or questionnaire does not ask for personal identifiers or any information that may be used to identify you. However, if you choose to participate in the study, you understand that your responses to the survey questions will be stored, and can be accessed, in the USA. The security and privacy policy for the websurvey company can be found at the following link: $\underline{h t t p: / / w w w . q u a l t r i c s . c o m / s e c u r i t y-s t a t e m e n t / '] ~}$

Incentives to Participate: Although we appreciate the contributions of participants in our research, you will not be paid for your participation in this study. However, you will receive $1 \%$ course credit for your Introductory Psychology course. If you would prefer to walk through the study - that is, if you would like to observe the research process but not fill out any questionnaires or provide any personal data - you will still be given $1 \%$ course credit. Note that while you can take part in as many psychology research studies as you wish, there is a limit in the course credit you can earn in any one term. This limit is in your syllabus.

Voluntary Nature of Participation: Participation in this study is voluntary. If you decide to participate, you are free to withdraw your consent and to stop your participation at any time without penalty or loss of benefits to which you are allowed. Should you withdraw from the study, you will still be given your $1 \%$ course credit (provided you have not already received the maximum allotted for research participation).

Dissemination of Results: The results of this study will form part of Sara Cowan's PhD dissertation. As well, results may be shared with others at scholarly meetings or as part of published papers. However, all information will be presented in aggregate form. That is, none of your individual information will be identifiable in any way. Anonymized data may be provided to other researchers for the purpose of study or verification of results; any data that is shared will NOT include the names of ANY participants.

Questions about the Study: If you have any questions about the research now, please ask. If you have questions later about the research, you may contact: Sara Cowan 416-979-5000 x 2190, sara.cowan@ psych.ryerson.ca ; Dr. Tara Burke, 416-979-5000, ex. 6519, tburke@psych.ryerson.ca

If you have questions regarding your rights as a human subject and participant in this study, you may contact the Ryerson University Research Ethics Board for information: Lynn Lavallée, PhD., Research Ethics Board, c/o Office of the Vice President, Research and Innovation, 
Ryerson University, 350 Victoria Street, Toronto, ON M5B 2K3, 416-979-5042, rebchair@ryerson.ca

If you any have questions about receiving your Psychology 102/202 credit for participation please contact: thepool@ psych.ryerson.ca

Agreement: Your signature below indicates that you have read the information in this agreement and have had a chance to ask any questions you have about the study. Your signature also indicates that you agree to be in the study and have been told that you can change your mind and withdraw your consent to participate at any time.

You have been told that by signing this consent agreement you are not giving up any of your legal rights.

Name of Participant (please print)

Signature of Participant

Date

Signature of Investigator

Date 


\section{INFORMED CONSENT DOCUMENT}

Title of Study: Criminal Investigation 1

Investigators: Sara Cowan, MA; Christian A. Meissner, Ph.D.

This form describes a research project. It has information to help you decide whether or not you wish to participate. Research studies include only people who choose to take part-your participation is completely voluntary. Please discuss any questions you have about the study or about this form with the project staff before deciding to participate.

\section{Introduction}

The purpose of this study is to examine how individuals determine the guilt or innocence of a suspect in a crime scenario. You are being invited to participate in this study because you are a psychology or communications student that is at least 18 years of age. You should not participate if you are not at least 18 years old.

\section{Description of Procedures}

If you decide to participate in this research, you will be asked to do the following: You will read some information about a criminal case, read a transcript of a police interview with a suspect, and answer some questions about your thoughts about the case. Your participation will last for up to 60 minutes.

\section{Risks or Discomforts}

While participating in this study you may experience the following risks or discomforts: Occasionally people feel uncomfortable when answering questions that ask about attitudes toward controversial social issues (e.g., affirmative action). While we do not anticipate that any of the questions you will be responding to are controversial, if any aspect of this study makes you feel uncomfortable, you may choose not to answer certain questions, or to withdraw from the study at any time without penalty.

\section{Benefits}

If you decide to participate in this study, there may be a direct benefit to you. We anticipate that you will benefit from this study by learning about how psychology research is conducted. When the session is over, we will describe the purpose and hypotheses of the study to you in more detail. Also, once we have analysed the data (toward the end of the term), you are welcome to view the results. It is hoped that the information gained in this study will benefit society by providing a better understanding about how individuals form impressions about criminal suspects.

\section{Costs and Compensation}

You will not have any costs from participating in this study. You will be compensated for participating in this study, through course credit. You will receive 2 SONA credits for your participation in the experiment.

\section{Alternatives to Participation}

You do not have to participate in this study. Your course syllabus describes alternatives to participation in research studies for earning this same course credit.

\section{Participant Rights}


Participating in this study is completely voluntary. You may choose not to take part in the study or to stop participating at any time, for any reason, without penalty or negative consequences.

Your choice of whether or not to participate (or stop participating at any time) will have no impact on you as a student in any way. You can skip any questions on the questionnaires that you do not wish to answer.

If you have any questions about the rights of research subjects or research-related injury, please contact the IRB Administrator, (515) 294-4566, IRB@iastate.edu, or Director, (515) 294-3115, Office for Responsible Research, Iowa State University, Ames, Iowa 50011.

\section{Confidentiality}

Records identifying participants will be kept confidential to the extent permitted by applicable laws and regulations and will not be made publicly available. However, federal government regulatory agencies, auditing departments of Iowa State University, and the Institutional Review Board at Iowa State University (a committee that reviews and approves human subject research studies) may inspect and/or copy study records for quality assurance and data analysis. These records may contain private information.

To ensure confidentiality to the extent permitted by law, the following measures will be taken: All data will be stored in a secure environment (within a locked filing cabinet, and on passwordprotected computers), and will only be analyzed across group averages. Your responses in this research will be confidential; your name may be linked to your responses. As such, your responses are not anonymous. Any materials that include your name-including this consent form-will be stored confidentially for a minimum of 5 years. Only the investigator and her faculty supervisor will have access to this information. Participants' identity will be kept confidential when the results are disseminated for publication.

\section{Questions}

You are encouraged to ask questions at any time during this study. For further information about the study, contact Sara Cowan at scowan@iastate.edu or sara.cowan@ psych.ryerson.ca. You may also contact her faculty supervisor at Iowa State University, Chris A. Meissner, Ph.D. at cameissn@iastate.edu.

\section{Consent and Authorization Provisions}

Your signature indicates that you voluntarily agree to participate in this study, that the study has been explained to you, that you have been given the time to read the document, and that your questions have been satisfactorily answered. You will receive a copy of the written informed consent prior to your participation in the study.

Participant's Name (printed)

Participant's Signature

Date 


\section{Appendix C: Original Reconsent Form for Experiment 1}

Agreement: Your signature indicates that you voluntarily agree to participate in this study, that the study has been explained to you, that you have been given the time to read the document, and that your questions have been satisfactorily answered. Your signature also indicates that you agree to allow the experimenter to retain the data collected in the study now that you have full knowledge of the purpose of this study. You have been given a copy of this agreement.

You have been told that by signing this consent agreement, you are not giving up any of your legal rights.

Participant's Name (printed)

Participant's Signature

Date 


\section{Appendix D: Revised Reconsent Form for Experiment 1 (September, 2015) \\ Reconsent - Criminal Investigation 1}

Agreement: Your signature below indicates that you have read the information in this debriefing form and have had a chance to ask any questions you have about the study. Your signature also indicates that you agree to allow the experimenter to retain the data collected in the study now that you have full knowledge of the purpose of this study. You have been given a copy of this agreement.

You have been told that by signing this consent agreement, you are not giving up any of your legal rights.

\section{Master List (CHECK ONE BOX)}

The researcher CAN include my name on the Master List The researcher CANNOT include my name on the Master List

\section{Questionnaire Responses (CHECK ONE BOX)}

The researcher CAN analyze my questionnaire responses The researcher CANNOT analyze my questionnaire responses

Name of Participant (please print)

Signature of Participant

Date

Signature of Investigator

Date 


\title{
Appendix E: Experiment 1 Materials
}

\section{Police Summary Report; Identical For All Conditions}

\author{
Supplemental Seattle Police Division \\ Crime Report $\quad$ Form 38.3 Rev. 5/09
}

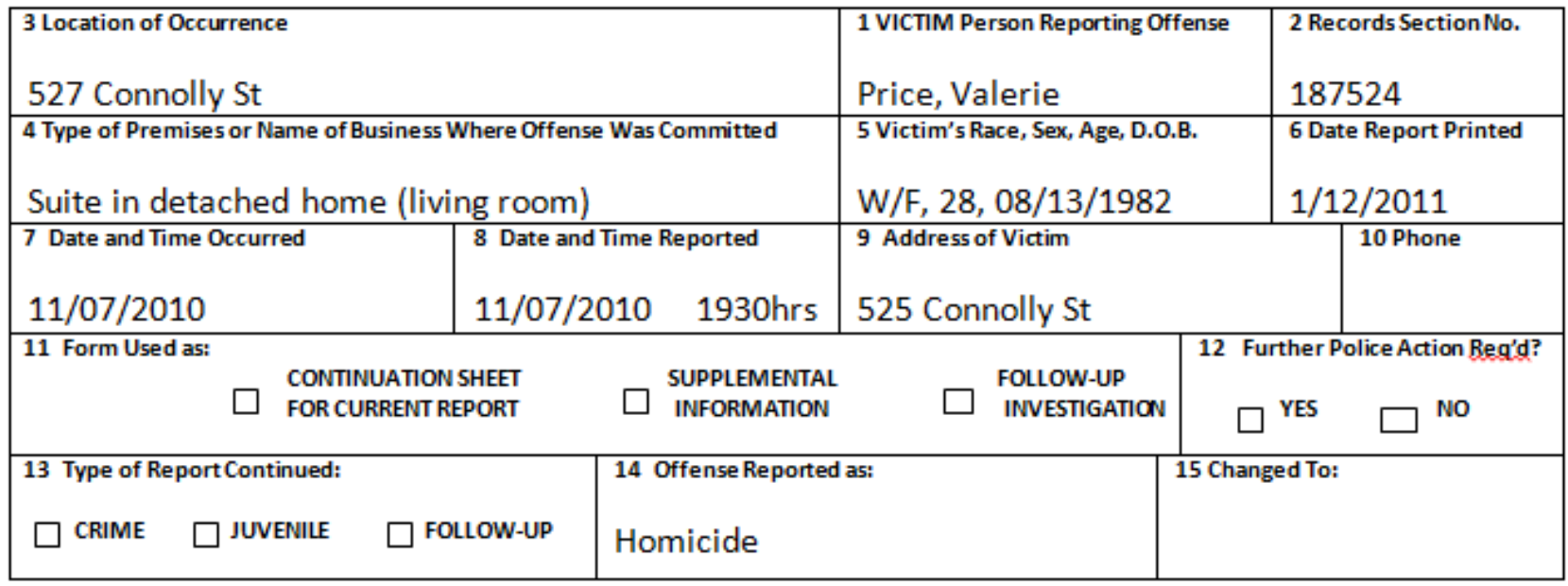

\section{NARRATIVE:}

On Sunday November 7, 2010, at approximately 1915 hours, a 911 distress call was received from Janice Parker of 525 Connelly St, reporting a scream and gunshots from the next door residence (527 Connelly St.) and an unidentified male fleeing the residence. Dispatch contacted Officers Fisher and Balfour (11 Division), and dispatched them to the scene. After entering and securing the premises, Fisher called 11 Division to report an apparent homicide at 527 Connelly St. The victim was found on the floor of the living room and it appeared that she had been shot twice in the chest. EMT personnel confirmed the victim was DOA. The investigation was then assigned to Detective Bailey, who prior to leaving Southwest Precinct, contacted Coroner's Investigator, T. Stanfield and Evidence Technician, B. Alexander.

Upon arrival at 527 Connolly St at approximately 2000 hours, Detective Bailey was met by Officer Fisher. Officer Fisher stated that he and Officer Balfour were dispatched to the scene in response to a 911 call. When the officers first arrived, they were met by personnel from No. 6 fire pumper and life squad No.2. The officers were told that the victim showed no signs of life and the death appeared to be a homicide. They were also told that the firefighters first found the victim lying face down on the living room floor. The victim, later identified as Valerie Price, was then turned over onto her back by Fire Department personnel as they attempted to render aid. Nothing else in the apartment was touched by Fire Department personnel. 
Officer Fisher also stated that after he viewed the body and carefully checked the other rooms in the apartment, the scene was secured and protected. Officer Fisher then contacted

Southwest Precinct for assistance. While waiting for the arrival of the detectives, Officer Fisher interviewed Janice Parker, the neighbour who placed the 911 call in the adjacent home at 525 Connelly St. During this time, Officer Balfour remained at the crime scene and ensured that nothing was disturbed. In the interview with Officer Fisher, Ms. Parker reported hearing a scream from the residence, followed by 2 or 3 gunshots. When she looked outside her window, she saw a male running down the street, wearing blue jeans, a green jacket with two reflective stripes down each arm, and a black baseball cap with a picture or logo on the front. The male was approximately 5'10" to 6'1" tall, average build, with no facial hair, between 30 to 40 years old. It was dusk, so she did not get a good look at the suspect and was not able to say for sure what race the man was.

\section{EVIDENCE:}

All evidence collected at the crime scene was collected by Evidence Technician Alexander.

\section{ADDITIONS:}

01/12/2011: In response to media appeals for information about the case, James Hearn (517 Taunton St) contacted $11^{\text {th }}$ District to report that the description of the culprit matched a nearby neighbor, Robert Harris (532 Taunton St). Officers Jiminez and Robertson were sent to conduct a preliminary interview with Harris. In the hallway of his home the officers observed a black baseball cap with an Arizona Cardinals logo and a green jacket with reflective stripes hanging on a peg. These items of clothing also match the description of the clothing worn by the culprit. The officers observed that Harris appeared nervous during the encounter, and believed that he was not forthcoming in his answers to their questions. As a result, officers determined Harris may be a person of interest and decided to take Mr. Harris in for secondary questioning with a Detective at the police station. 


\section{Suspect Interrogation Transcript}

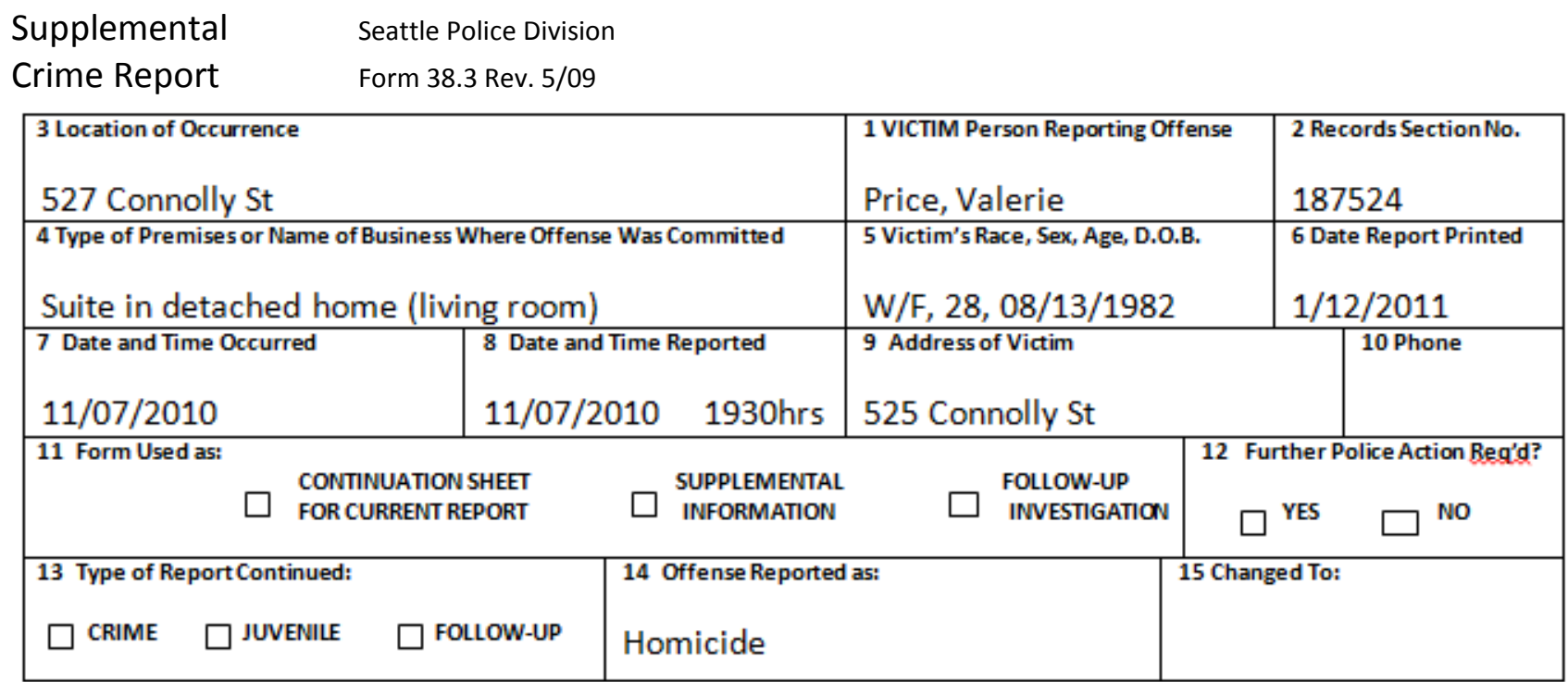

\section{INTERROGATION OF ROBERT HARRIS, 1/12/2011, 11:01PM:}

Det. Bailey: Could you state your full name for the records?

Harris: My name is Robert Harris.

Det. Bailey: Thank you. And where do you live, Mr. Harris?

Harris: 532 Taunton St.

Det. Bailey: And how long have you lived there?

Harris: About 5 years or so.

Det. Bailey: Good, thank you. We are investigating the murder of one of your neighbours,

Valerie Price, on November $7^{\text {th }}, 2010$, approximately two months ago. This is a photo of her [shows Harris the photo]. Did you know the victim at all, Mr. Harris?

Harris: Yes. 
Det. Bailey: How well did you know her?

Harris: Not very. I mean, I'd recognize her, we'd say hi if we saw each other on the street, but that's about it.

Det. Bailey: How long had you known her?

Harris: I don't know, maybe a couple of years, but I'm really not sure.

Det. Bailey: And how would you characterize your relationship with her?

Harris: I don't know. Acquaintances, I guess.

Det. Bailey: And have you ever been inside her home?

Harris: No.

Det. Bailey: Could you detail for me, to the best of your ability, your whereabouts on November 7, 2010, the day of Ms. Price's murder two months ago. Start at the beginning of your day and continue until the end of that day. Keep in mind that we will be verifying your statement and any lies or omissions that we note will cast further suspicion on you. Murder is a serious crime, Mr. Harris, so really, it's in your best interest to be as honest and forthcoming as possible.

\section{VERSION 1 (Morally Undesirable)}

Harris: I got up between 8:30 and 9, spent the morning at home working on a project for work, watched the football game in the afternoon, then made dinner at home.

Det. Bailey: OK, were you still at home that same evening?

Harris: No.

Det. Bailey: Where were you that evening between 7 and 7:30PM, Mr. Harris?

Harris: I was at the home of my friend Michael Beauregard and his wife Kaitlynn. I think I got 
there at about twenty to seven, maybe quarter to.

Det. Bailey: Were they home?

Harris: Michael is away on a business trip, but Kaitlynn was there.

Det. Bailey: OK, we will be checking on that. What were you doing there?

Harris: That night I was in bed with Kaitlynn. We've been having an affair.

Det. Bailey: What time did you return home?

Harris: I think I left around 11:30 or so, and was home by 11:45.

Det. Bailey: And you're sure that is the time you returned home? It could not have been any earlier?

Harris: Yes, I'm positive.

Det. Bailey: Thank you. We're going to need you to provide us with a list of individuals who could support your statement and any physical evidence that could confirm what you say. After that, you are free to go, though we may need to interview you again.

END OF INTERVIEW

\section{VERSION 2 (Morally Neutral)}

Harris: I got up between 8:30 and 9, spent the morning at home working on a project for work, watched the football game in the afternoon, then made dinner at home.

Det. Bailey: OK, were you still at home that same evening?

Harris: No.

Det. Bailey: Where were you that evening between 7 and 7:30PM, Mr. Harris?

Harris: I was at the home of my friend Michael Beauregard and his wife Kaitlynn. I think I got 
there at about twenty to seven, maybe quarter to.

Det. Bailey: Were they home?

Harris: Michael is away on a business trip, but Kaitlynn was there.

Det. Bailey: OK, we will be checking on that. What were you doing there?

Harris: That night I was picking up some DVDs I let Michael and Kaitlynn borrow.

Det. Bailey: What time did you return home?

Harris: I think I left around 11:30 or so, and was home by 11:45.

Det. Bailey: And you're sure that is the time you returned home? It could not have been any earlier?

Harris: Yes, I'm positive.

Det. Bailey: Thank you. We're going to need you to provide us with a list of individuals who could support your statement and any physical evidence that could confirm what you say. After that, you are free to go, though we may need to interview you again.

END OF INTERVIEW

\section{VERSION 3 (Morally Desirable)}

Harris: I got up between 8:30 and 9, spent the morning at home working on a project for work, watched the football game in the afternoon, then made dinner at home.

Det. Bailey: OK, were you still at home that same evening?

Harris: No.

Det. Bailey: Where were you that evening between 7 and 7:30PM, Mr. Harris?

Harris: I was at the home of my friend Michael Beauregard and his wife Kaitlynn. I think I got 
there at about twenty to seven, maybe quarter to.

Det. Bailey: Were they home?

Harris: Michael is away on a business trip, but Kaitlynn was there.

Det. Bailey: OK, we will be checking on that. What were you doing there?

Harris: That night I was helping Michael and Kaitlynn move, loading boxes and furniture on the truck.

Det. Bailey: What time did you return home?

Harris: I think I left around 11:30 or so, and was home by 11:45.

Det. Bailey: And you're sure that is the time you returned home? It could not have been any earlier?

Harris: Yes, I'm positive.

Det. Bailey: Thank you. We're going to need you to provide us with a list of individuals who could support your statement and any physical evidence that could confirm what you say. After that, you are free to go, though we may need to interview you again. END OF INTERVIEW

\section{VERSION 4 (No-Alibi Control)}

Harris: Let me think. [PAUSE] That was about two months ago, so I'm having trouble remembering. What day of the week was that?

Det. Bailey: It was a Sunday.

Harris: In that case, if there was a football game on, I was probably watching that in the afternoon. 
Det. Bailey: Where were you that evening between 7 and 7:30PM, Mr. Harris?

Harris: Without checking my calendar, I really can't be sure. I just can't remember where I was that night.

Det. Bailey: Were you at home, or were you out that evening?

Harris: I just don't know. Some Sunday evenings I stay in, sometimes I go out.

Det. Bailey: So you do not have regular Sunday evening activities?

Harris: No. It varies.

Det Bailey: What kinds of activities might you do on a Sunday night?

Harris: Maybe a drink with friends, or a dinner date.

Det. Bailey: When you do go out on Sundays, what time do you usually return home?

Harris: It depends, usually not after 11:30 or so.

Det. Bailey: OK, we will be continuing our investigation. You are free to go, though we may need to interview you again.

END OF INTERVIEW 


\section{Supplemental Evidence Report: Strong Evidence Condition}

Evidence Seattle Police Division

Report Form 38.3 Rev. 5/09

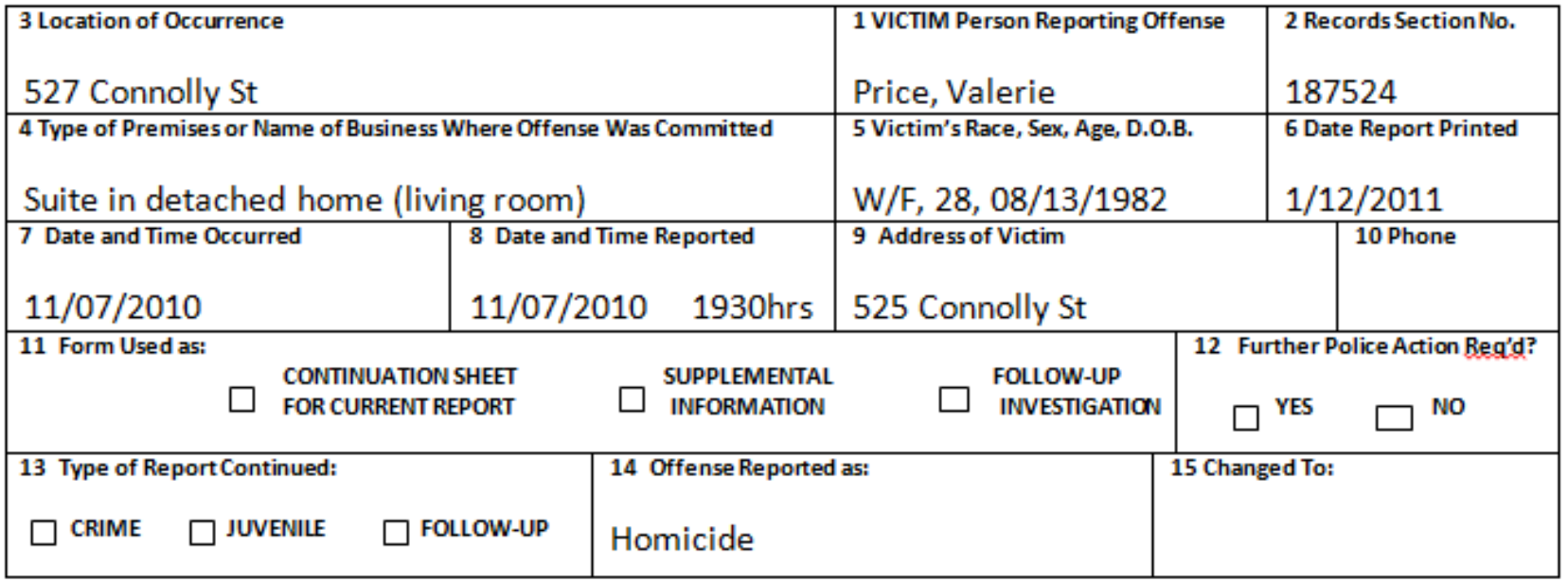

\section{EVIDENCE - ALIBI}

Suspect (Robert Harris) indicated that he parked his car at the Standard Parking lot at 721 Pine St. Lot is approximately 4.5 miles $(7.2 \mathrm{~km})$ from crime scene. Security video has been obtained from the lot. Video appears to show Harris parking in the lot at 6:48pm and departing at $11: 27 p m, 11 / 07 / 2010$.

Harris has also provided contact information for Kaitlynn Beauregard (alibi witness). Det. Bailey will follow up. 


\section{Supplemental Evidence Report: Weak Evidence Condition}

Evidence Seattle Police Division

Report Form 38.3 Rev. 5/09

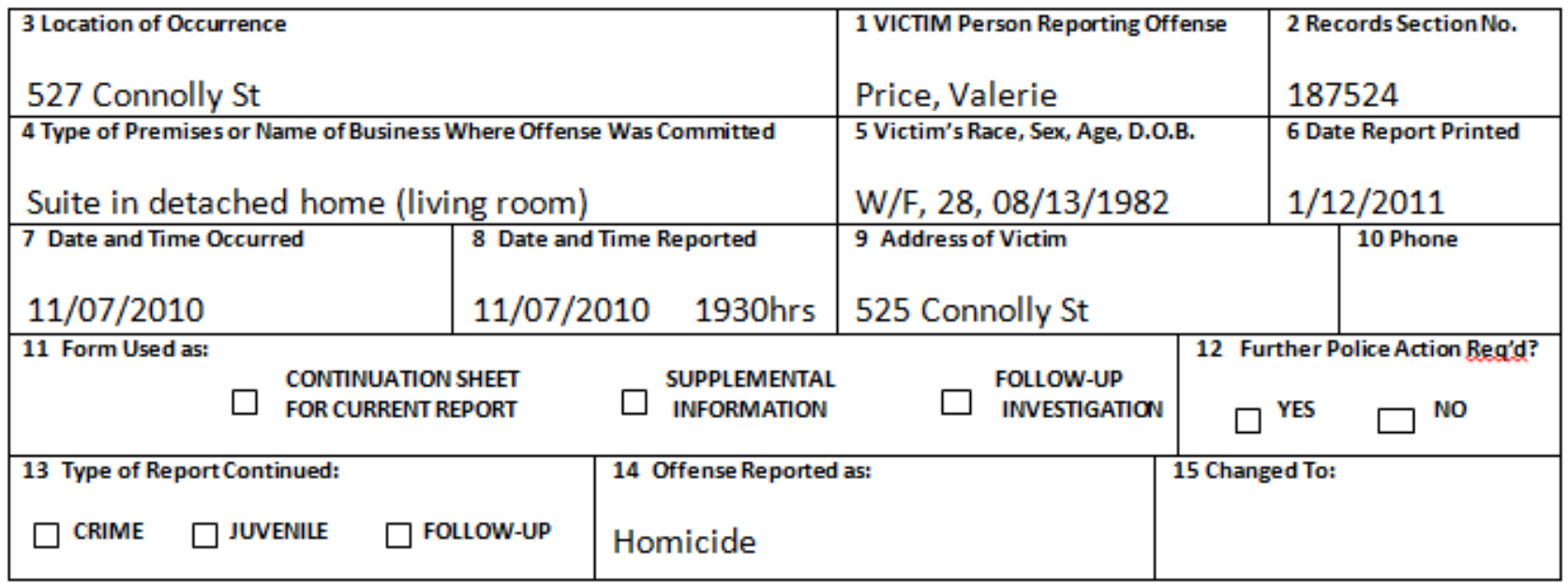

\section{EVIDENCE - ALIBI}

Suspect (Robert Harris) indicated that he parked his car at the Standard Parking lot at 721 Pine St. Lot is approximately 4.5 miles $(7.2 \mathrm{~km})$ from crime scene. Harris has provided a receipt showing arrival at the lot at 6:48pm, departure at $11: 27 \mathrm{pm}, 11 / 07 / 2010$. No security video has been obtained from the lot; cameras were not operating.

Harris has also provided contact information for Kaitlynn Beauregard (alibi witness). Det. Bailey will follow up. 


\section{Supplemental Evidence Report: No Evidence Condition}

Evidence Seattle Police Division

Report Form 38.3 Rev. 5/09

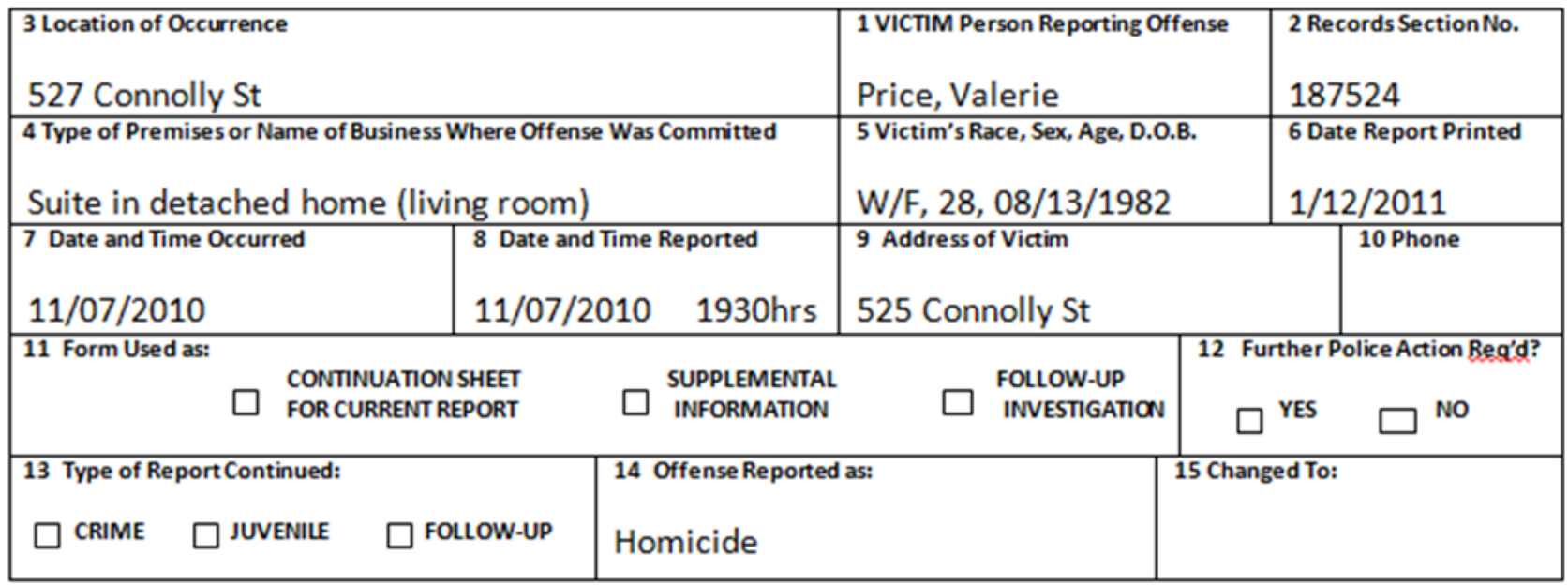

\section{EVIDENCE - ALIBI}

Suspect (Robert Harris) indicated that he parked his car at the Standard Parking lot at 721 Pine St. Lot is approximately 4.5 miles $(7.2 \mathrm{~km})$ from crime scene. No security video has been obtained from the lot; cameras were not operating. Receipt has been lost.

Harris has also provided contact information for Kaitlynn Beauregard (alibi witness). Det. Bailey will follow up. 
Appendix F: Experiment 1 Questionnaire

Valerie Price Case

NOTE: PARTICIPANTS IN NO-ALIBI CONTROL WILL NOT BE ASKED ABOUT THE ALIBI

1. What is your age? Years

2. In terms of your gender, you would self-identify as (choose all that apply):

$\square$ Female

$\square$ Male

$\square$ Trans*

$\square$ Queer

Other, please specify

3. What is your ethnicity (choose all that apply)?

- American Indian or Alaska Native

- First Nations Canadian

- Black or African American

- East Asian

- Hispanic or Latino/a

- Native Hawaiian or Other Pacific Islander

- West Asian/Middle Eastern

- White

- Other, please specify

Please answer the following questions as best you can with the limited information from the police file.

4. How honest do you think Robert Harris is being in his description of his whereabouts on the evening in question?

$\begin{array}{ccccccc}1 & 2 & 3 & 4 & 5 & 6 & 7 \\ \begin{array}{c}\text { Not at all } \\ \text { honest }\end{array} & & \begin{array}{c}\text { Somewhat } \\ \text { honest }\end{array} & & \begin{array}{c}\text { Very } \\ \text { honest }\end{array} & \end{array}$

5. How confident are you in your decision for Question \#4?

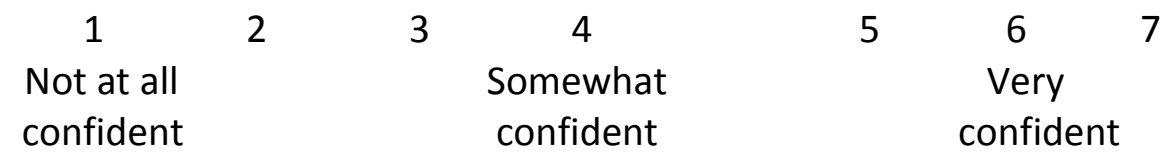


6. How accurate do you think Robert Harris' statement regarding his whereabouts on the evening in question is? In other words, how well does his statement match his activities on the night in question?

$\begin{array}{ccccccc}1 & 2 & 3 & \begin{array}{c}4 \\ \text { Somewhat } \\ \text { accurate }\end{array} & 5 & 6 & \begin{array}{c}7 \\ \text { Very all }\end{array} \\ \text { accurate } & & & & & \text { accurate }\end{array}$

7. How confident are you in your decision for Question \#6?

$\begin{array}{ccccccc}1 & 2 & 3 & 4 & 5 & 6 & 7 \\ \begin{array}{c}\text { Not at all } \\ \text { confident }\end{array} & & & \begin{array}{c}\text { Somewhat } \\ \text { confident }\end{array} & & & \begin{array}{c}\text { Very } \\ \text { confident }\end{array}\end{array}$

8. When Robert Harris said that he was somewhere else when the crime was committed, his description of what he was doing at the time was probably:

The truth; he was telling the police where he was at the time A lie; he was deliberately giving false information to police An error; he was trying to tell the truth, but was mistaken about his whereabouts

9. Overall, how strong do you think the evidence is against Robert Harris?

$\begin{array}{ccccccc}1 & 2 & 3 & 4 & 5 & 6 & 7 \\ \begin{array}{c}\text { Not at all } \\ \text { strong }\end{array} & & & \begin{array}{c}\text { Somewhat } \\ \text { strong }\end{array} & & & \begin{array}{c}\text { Very } \\ \text { strong }\end{array}\end{array}$

10. Based on the evidence provided to you, how likely do you think it is that Robert Harris committed the crime?

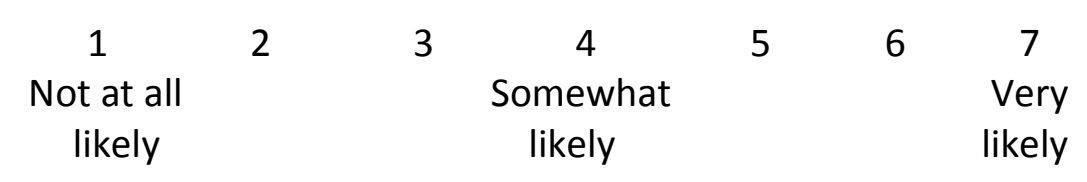

11. If I was asked to provide a verdict in the case, I would vote that Robert Harris is:

_ Guilty Innocent

12. In terms of personality, how similar do you think you are to Robert Harris?

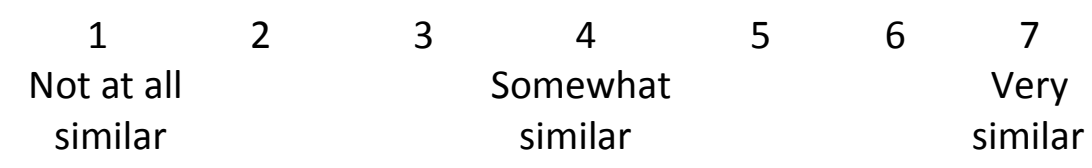


13. How much do you like Robert Harris?

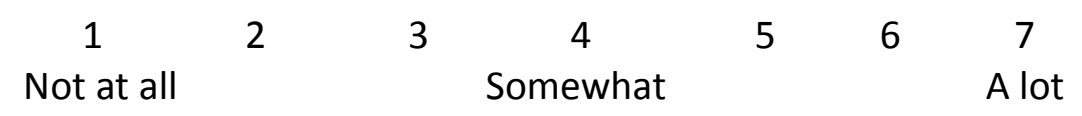

14. Overall, I think that Robert Harris is:

1

23

4

5

6

7

8

9

10

11

A very

Neither

A very

bad

good

good

person

nor bad

person

15. What did Robert Harris say he was doing at the time of the crime?

16. Have you ever engaged in the behavior(s) you described in the previous question?<smiles>CC[AsH2]C</smiles>

17. Did Robert Harris provide any evidence to support his statement that he was somewhere else when the crime was committed? If so, please describe.

18. Regarding Robert Harris' activities at the time of the crime, you would evaluate his 
behavior as:

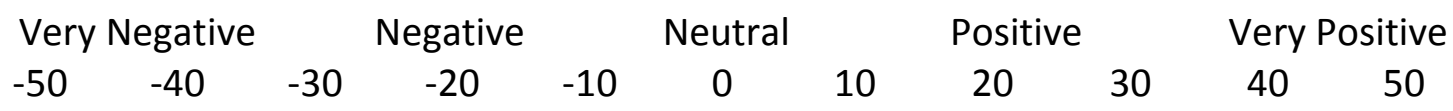

19. Regarding Robert Harris' activities at the time of the crime, if you found out that an adult male in North America did this at least once, your evaluation of that person would be:

\begin{tabular}{|c|c|c|c|}
\hline Very Negative & & oati & \\
\hline$-50 \quad-40$ & -30 & -20 & \\
\hline
\end{tabular}

20. Please write below, in your own words, how you define the term ALIBI.

21. Please provide any general comments you have about the case. 


\section{Appendix G: Authoritarianism-Conservatism-Traditionalism Scale (Duckitt et al., 2010)}

\section{Please indicate your response, using the scale below.}

$\begin{array}{lcccccc}1 & 2 & 3 & 4 & 5 & 6 & 7 \\ \text { Strongly } & \text { Moderately } & \text { Slightly } & \text { Neither Disagree } & \text { Slightly } & \text { Moderately } & \text { Strongly } \\ \text { Disagree } & \text { Disagree } & \text { Disagree } & \text { Nor Agree } & \text { Agree } & \text { Agree } & \text { Agree }\end{array}$

\section{Conservatism ("Authoritarian Submission")}

1. It's great that many young people today are prepared to defy authority.

2. What our country needs most is discipline, with everyone following our leaders in unity.

3. Students at high schools and at university must be encouraged to challenge, criticize, and confront established authorities.

4. Obedience and respect for authority are the most important virtues children should learn.

5. Our country will be great if we show respect for authority and obey our leaders.

6. People should be ready to protest against and challenge laws they don't agree with.

\section{Traditionalism}

7. Nobody should stick to the "straight and narrow." Instead people should break loose and try out lots of different ideas and experiences.

8. The "old-fashioned ways" and "old-fashioned values" still show the best way to live.

9. God's laws about abortion, pornography, and marriage must be strictly followed before it is too late.

10. There is absolutely nothing wrong with nudist camps.

11. This country will flourish if young people stop experimenting with drugs, alcohol, and sex, and pay more attention to family values.

12. There is nothing wrong with premarital sexual intercourse.

\section{Authoritarianism ("Authoritarian Aggression”)}

13. Strong, tough government will harm not help our country.

14. Being kind to loafers or criminals will only encourage them to take advantage of your weakness, so it's best to use a firm, tough hand when dealing with them.

15. Our society does NOT need tougher government and stricter laws.

16. The facts on crime and the recent public disorders show we have to crack down harder on troublemakers, if we are going preserve law and order.

17. Our prisons are a shocking disgrace. Criminals are unfortunate people who deserve much better care, instead of so much punishment.

18. The way things are going in this country, it's going to take a lot of "strong medicine" to straighten out the troublemakers, criminals, and perverts. 


\section{Appendix H: Petition Website for Experiment 1}

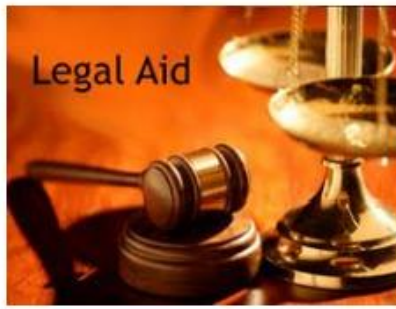

Petitioning Legal Aid Washington

Defend Robert Harris in Court

Robert Harris has been accused of killing his neighbor, Valerie Price, a

charge he strongly denies. The

costs of his legal defense have

already used up his limited savings,

and he is now unable to afford a

lawyer. He has applied to Legal Aid

Washington to be appointed a

lawyer, but the agency has yet to

decide if he qualifies for help.

Everyone is entitled to a good legal

defense. Please sign this petition

to ask Legal Aid Washington to

take Robert Harris' case.

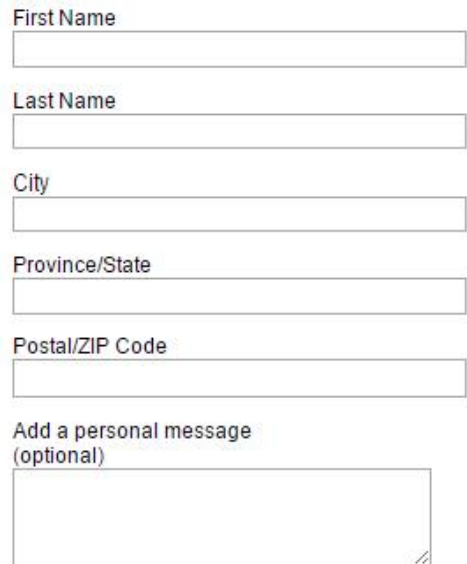

Sign The Petition Now $>$

By signing, you accept

importantpetitions.com's terms of

service and privacy policy. 


\section{Appendix I: Experiment 1 Manipulation Checks}

Participants who were not in the Control condition were asked to rate the behaviour that the suspect described in his alibi on a Likert scale from 1 (Very Negative) to 11 (Very Positive). Alibi Moral Desirability significantly affected participants' judgments about the behaviour, $F(2$, $261)=121.62, p<.001, \eta_{p}^{2}=.48$. No differences were observed between ratings of participants at Iowa State or Ryerson, $F(1,261)=1.59, p=.208, \eta_{\mathrm{p}}^{2}=.01$, and there was no significant interaction between the two variables, $F(2,261)=1.33, p=.266, \eta_{\mathrm{p}}^{2}=.01$. Follow-up $t$-tests for all participants together showed significant differences between all groups. The morality of the behaviour was rated as significantly lower for Undesirable $(M=3.07 \mathrm{SD}=1.75)$ than for Neutral alibis $(M=6.25 \mathrm{SD}=1.66), t(176)=-12.46, p<.001, d=-1.87$. It was also lower for Undesirable than for Desirable alibis $(M=6.90), t(174)=-14.07, p<.001, d=-2.12$. Finally, the Neutral alibi was viewed less positively than the Desirable one, $t(174.74)=-2.46, p=.015, d=-$ .40 .

To ensure that the Morally Desirable, Morally Undesirable, and Neutral alibis were perceived as such by participants, the mean of participants' ratings of their feelings about the suspect's behaviour for each alibi was compared to the midpoint (i.e., 6). As no Location effects were observed above, results were not computed separately based on sample location. Results show that the Morally Desirable alibi was rated significantly higher than the midpoint, $t(88)=$ 4.56, $p<.001, d=0.48$, the Morally Undesirable alibi was rated as significantly lower than the midpoint, $t(86)=-15.62, p<.001, d=-1.67$, and the Neutral alibi was not significantly different from the midpoint, $t(90)=1.46, p=.149, d=0.15$. These results indicate that the manipulation operated as intended.

Participants who were not in the Control condition were also asked to indicate how they 
would judge an average adult male who had engaged in this behaviour on a scale from 1 (Very Negative) to 11 (Very Positive). As anticipated, these ratings were highly correlated with participants' ratings about the suspect's reported behaviour, $r=.79, p<.001$. Alibi Moral Desirability significantly affected this judgment as well, $F(2,258)=118.93, p<.001, \eta_{\mathrm{p}}^{2}=.48$. However, there was a significant main effect for Location, $F(1,258)=10.68, p=.001, \eta_{\mathrm{p}}^{2}=.04$, and a significant interaction between Location and Alibi Moral Desirability, $F(2,258)=3.55, p$ $=.030, \eta_{\mathrm{p}}^{2}=.03$. At Iowa State, the Morally Undesirable $(M=3.20, S D=1.71)$ alibi was viewed more negatively than the Neutral $(M=6.33, S D=1.48)$ alibi, $t(77)=-8.70, p<.001, d=-1.96$, or the Morally Desirable $(M=7.65, S D=1.88)$ alibi, $t(78)=-11.08, p<.001, d=-2.48$. As well, the Morally Desirable alibi was viewed more positively than the Neutral alibi, $t(77)=3.46, p=$ $.001, d=0.78$. Similarly, at Ryerson, the Morally Undesirable $(M=3.23, S D=1.59)$ alibi was viewed significantly more negatively than the Morally Neutral $(M=5.59, S D=1.64)$ alibi, $t(96)$ $=-7.20, p<.001, d=-1.46$, or the Morally Desirable $(M=6.34, S D=1.69)$ alibi, $t(92)=-9.19, p$ $<001, d=-1.90$. Also, the Morally Desirable alibi was viewed more positively than the Neutral alibi, using a Holm-Bonferroni correction, $t(96)=2.24, p=.027, d=0.45$.

Again, to ensure that all of the alibis were perceived by participants as anticipated, mean ratings were compared to the midpoint of the scale. At Iowa State, ratings of the actor were significantly higher than the midpoint for the Morally Desirable alibi, $t(39)=5.57, d=0.88$, significantly lower than the midpoint for the Morally Undesirable alibi, $t(39)=-10.34, p<.001$, $d=-1.63$, and not significantly different from the midpoint for the Neutral alibi, $t(38)=1.41, p=$ $.166, d=0.22$. At Ryerson, ratings of the actor were significantly lower than the midpoint for the Morally Undesirable alibi, $t(46)=-11.92, p<.001, d=-1.74$, but were not significantly different from the midpoint for the Morally Desirable, $t(46)=1.39, p=.173, d=0.20$, or Neutral alibis, 
$t(50)=-1.79, p=.079, d=-0.25$. Overall, the results of these manipulation checks suggest that the manipulation operated as intended. At Ryerson, the Morally Desirable alibi was not perceived as particularly positive, but it was seen more positively than the Neutral alibi. 


\section{Appendix J: Exploring Location Effects in Experiment 1}

\section{Participant Race/Ethnicity}

The sample at Ryerson was highly ethnically diverse. A sufficient number of participants self-identified as East Asian (28.4\%) to permit them to be included in analyses as a separate group, though responses from East Asian participants were not significantly different from other non-White participants in this sample for continuous dependent measures (see Table J1), verdict choice, $\chi^{2}(1)=.91, p=.414, \varphi=.09$, or willingness to sign the petition, $\chi^{2}(1)=1.41, p=.258, \varphi$ $=-.14$. Consequently, all non-White participants at Ryerson were included in a single group for the following analyses. Individuals who chose more than one of the pre-selected categories were included in the POC group, except for when they selected White and Other, and the other was a more specific identifier of national origin (e.g., Portuguese).

Table J1

Comparison of responses of participants who identified as East Asian or as another non-White group in the Ryerson sample

\begin{tabular}{lcccc}
\hline Dependent Measure & $d f$ & $t$ & $p$ & $d$ \\
\hline Suspect honesty & 105 & -1.25 & .213 & -0.25 \\
Confidence in suspect honesty & 105 & -.28 & .781 & -0.05 \\
Alibi accuracy* & 93 & -.28 & .778 & -0.06 \\
Confidence in alibi accuracy* & 93 & .59 & .560 & 0.12 \\
Strength of the evidence against the suspect & 105 & .03 & .973 & 0.01 \\
Likelihood of suspect guilt & 105 & .66 & .509 & 0.13 \\
\hline
\end{tabular}

Note. Dependent measures marked with an * did not include participants in the No-Alibi Control condition, as they were not asked to respond to this question.

Suspect honesty. Participants' ratings of alibi honesty varied as a function of race/ethnicity, $F(2,282)=4.25, p=.015, \eta_{\mathrm{p}}^{2}=.029$ (see Figure J1). Follow-up $t$-tests showed that POC at Ryerson rated the suspect's statement as significantly less honest than did White participants at Iowa State, $t(229)=2.88, p=.004, d=0.39$. Ratings by White participants at 
Ryerson did not differ from ratings by White participants at Iowa State, $t(176)=.55, p=.582, d$ $=0.09$, or from ratings by POC at Ryerson, $t(159)=1.68, p=.096, d=0.28$.

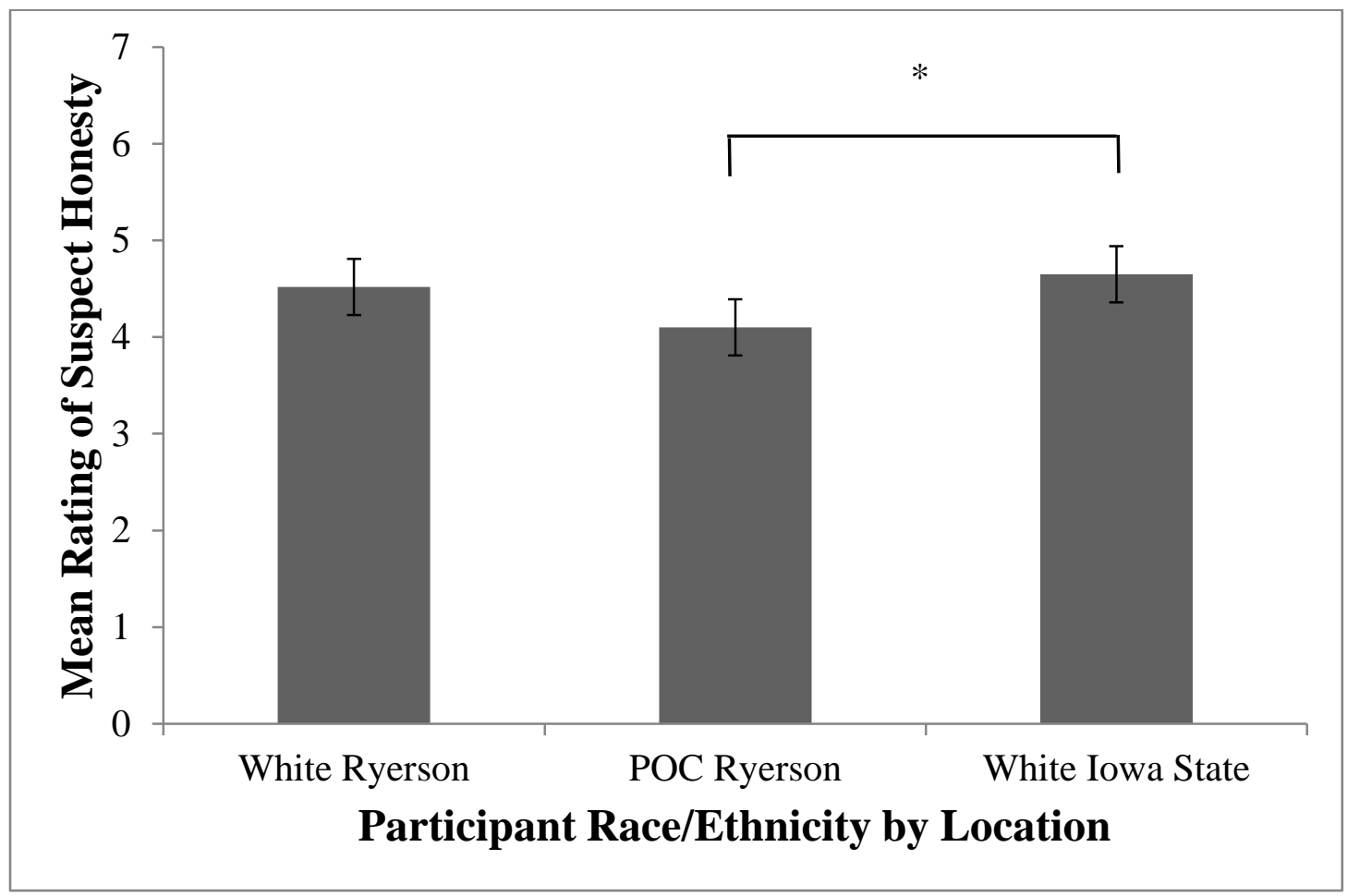

Figure J1

Comparison of mean ratings of suspect honesty by participants of different racial/ethnic groups at Ryerson and Iowa State.

Confidence in suspect honesty. No significant differences were found between racial/ethnic groups in self-reported confidence, $F(2,282)=.69, p=.505, \eta_{\mathrm{p}}^{2}=.005$.

Alibi accuracy. Ratings of alibi accuracy did not vary significantly as a function of participants' race/ethnicity, $F(2,248)=2.41, p=.092, \eta_{\mathrm{p}}^{2}=.019$.

Confidence in alibi accuracy. Participants' self-reported confidence in their ratings of the accuracy of the alibi statement were unaffected by race/ethnicity, $F(2,248)=1.35, p=.097$, $\eta_{\mathrm{p}}^{2}=.019$

Strength of the evidence against the suspect. Participants' ratings of the strength of the 
evidence against the suspect did differ significantly based on their racial/ethnic group, $F(2,281)$ $=9.16, p<.001, \eta_{\mathrm{p}}^{2}=.061$ (see Figure J2). As with ratings of suspect honesty, a significant difference was found between White participants at Iowa State and POC at Ryerson, $t(228)=-$ $4.39, p<.001, d=-0.58$, with higher ratings from $\mathrm{POC}$ at Ryerson. A non-significant trend suggested that White participants at Ryerson rated the evidence as weaker than POC at Ryerson, $t(159)=-2.23, p=.027, d=-0.37$. The difference between White participants at Iowa State and Ryerson was not significant, $t(175)=-1.04, p=.298, d=-0.17$.

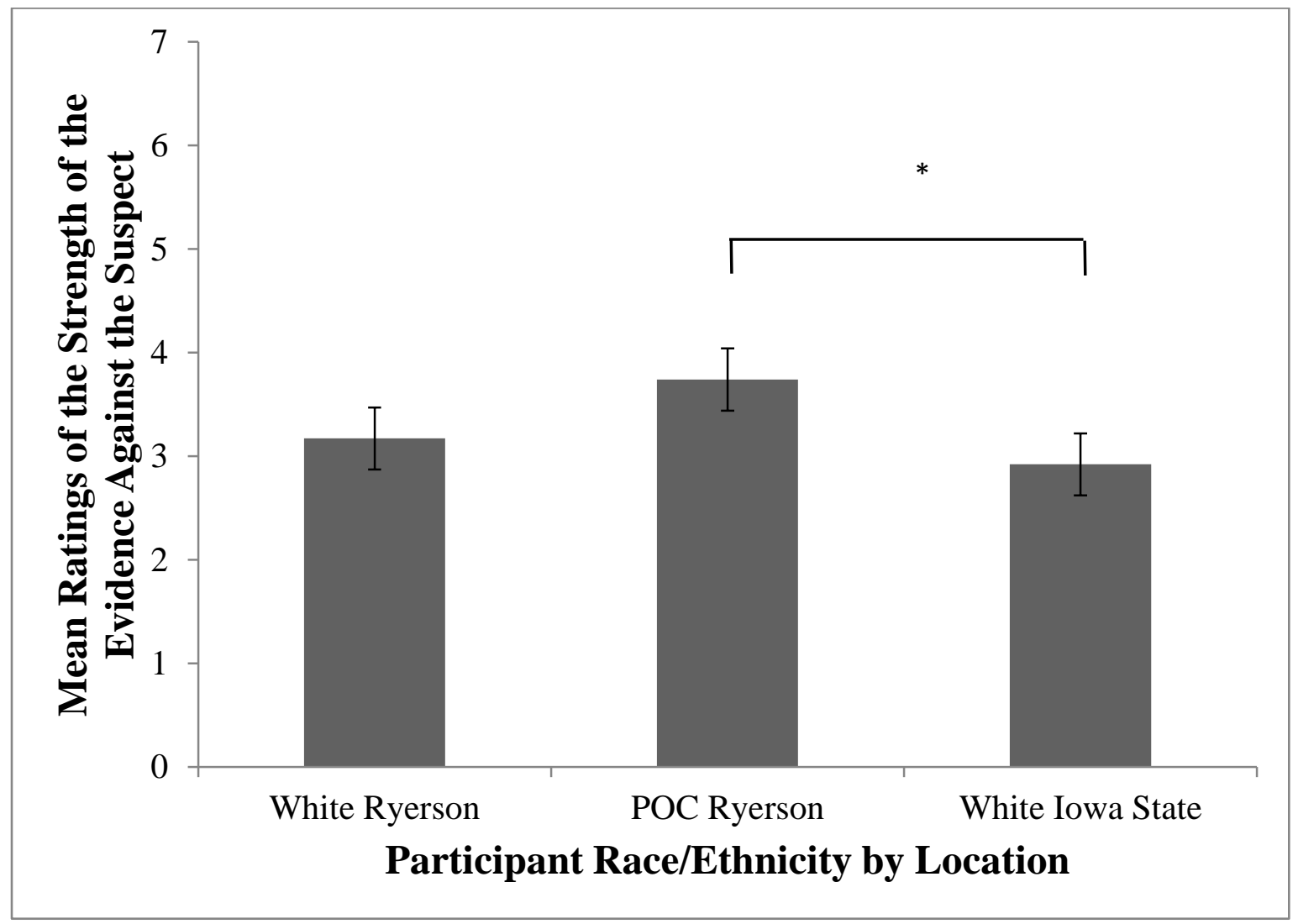

Figure J2

Comparison of mean ratings of the strength of the evidence against the suspect by participants of different racial/ethnic groups at Ryerson and Iowa State.

Likelihood of suspect guilt. Likelihood of suspect guilt ratings were also significantly affected by participants' race/ethnicity, $F(2,282)=6.07, p=.003, \eta_{\mathrm{p}}^{2}=.041$ (see Figure J3). As 
with earlier analyses in this section, the only significant difference between groups was between White participants at Iowa State and POC at Ryerson $t(229)=-3.61, p<.001, d=-0.48$, with POC at Ryerson providing higher estimates of the likelihood that the suspect was guilty. Ratings of White participants at Ryerson were not significantly different from ratings of $\mathrm{POC}$ at Ryerson, $t(91.46)=-1.40, p=.164, d=-0.25$, or from ratings of White participants at Iowa State, $t(176)=$ $-1.23, p=.219, d=-0.21$.

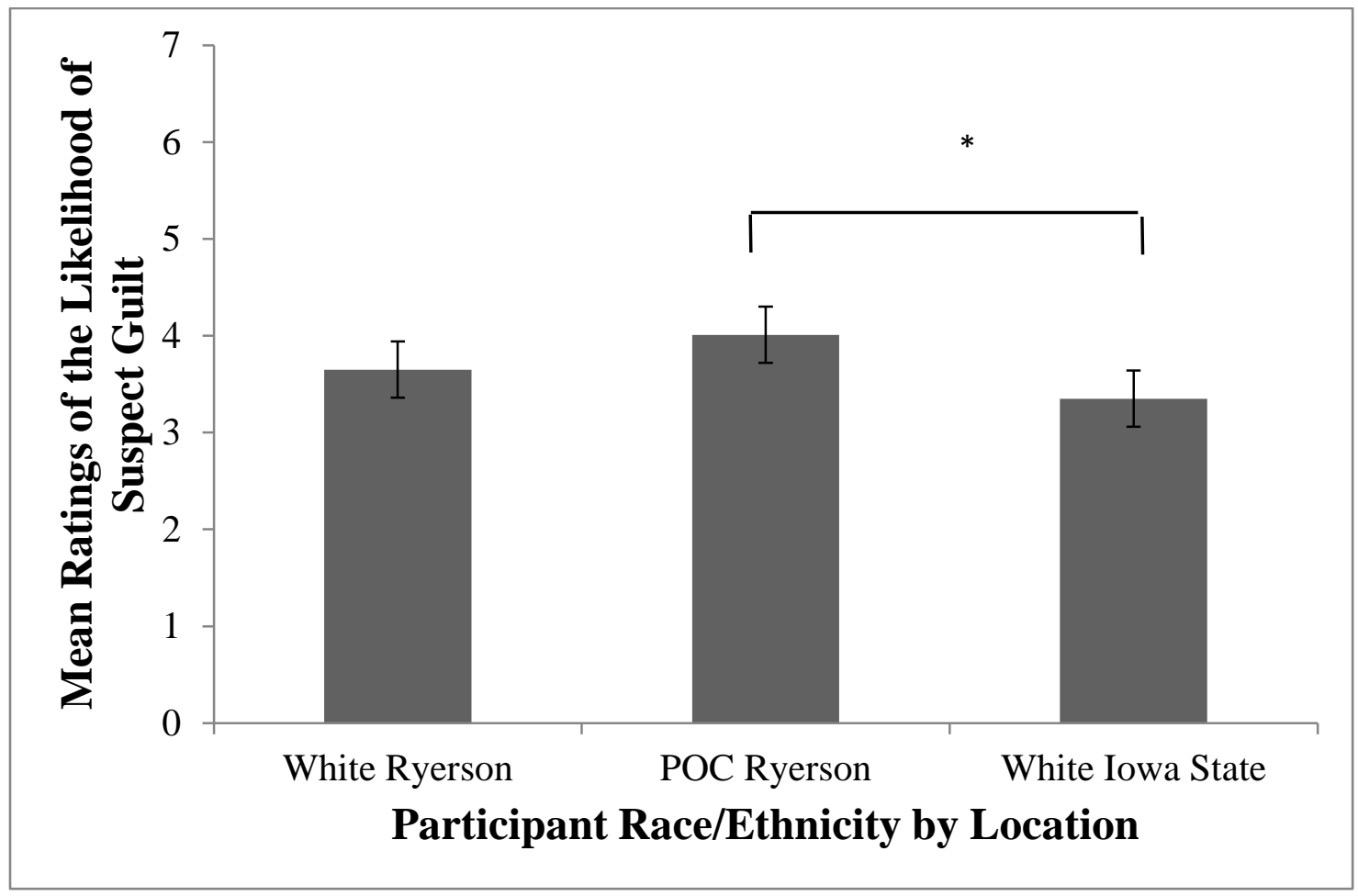

Figure J3

Comparison of mean ratings of the likelihood that the suspect is guilty by participants of different racial/ethnic groups at Ryerson and Iowa State.

Verdict choice. A chi-square was computed to determine if rates of guilty verdicts varied as a function of participants' race/ethnicity. This analysis indicated that verdicts were affected by race/ethnicity, $\chi^{2}(2)=6.28, p=.042, V=.15$. Follow-up analyses were conducted by testing the significance of the difference between independent proportions using the VassarStats website. 
Again, as with previous analyses, the only significant comparison was between White participants at Iowa State $(27 / 124 ; 21.8 \%$ guilty verdicts) and POC at Ryerson $(39 / 107 ; 36.4 \%$ guilty verdicts), $z=2.46, p=.014$. The rate of guilty verdicts for White participants at Ryerson $(14 / 54 ; 25.9 \%$ guilty verdicts) was not significantly different from POC at Ryerson, $z=1.34, p=$ .180 , or White participants at Iowa State, $z=0.61, p=.545$. Overall, the highest percentage of guilty verdicts was from POC at Ryerson, and the lowest was from White participants at Iowa State.

Willingness to sign the petition. Participants' willingness to sign the petition was independent of their race/ethnicity, $\chi^{2}(2)=.86, p=.669, V=.06$.

\section{Participant Gender}

Suspect honesty. A 2 (Participant Gender: Male, Female) X 2 (Location: Iowa State, Ryerson) ANOVA showed only a significant main effect for Location, $F(1,294)=9.55, p=$ $.002, \eta_{\mathrm{p}}^{2}=.031$. There was no significant effect of participant gender, $F(1,294)=.25, p=.615$, $\eta_{\mathrm{p}}^{2}=.001$. The interaction between Location and participant gender approached significance, $F(1,294)=3.56, p=.060, \eta_{\mathrm{p}}^{2}=.012$. As the purpose of these analyses is to investigate whether participant gender explains the differences in results based on Location, follow-up $t$-tests were used to compare the responses of men and of women at the different sample sites. Female participants' ratings of suspect honesty were the same at Iowa State $(M=4.55, S D=1.29)$ and at Ryerson $(M=4.32, S D=1.54), t(207)=1.11, p=.267, d=0.16$. However, for male participants, ratings of suspect honesty were significantly higher at Iowa State $(M=4.82, S D=$ 1.46) than at Ryerson $(M=3.86, S D=1.28), t(87)=3.02, p=.003, d=0.69$.

Confidence in suspect honesty. As with previous analyses, no significant effects were observed for participants' confidence in their judgments of suspect honesty: participant gender, 
$F(1,294)=.11, p=.740, \eta_{\mathrm{p}}^{2}<.001 ;$ Location, $F(1,294)=.59, p=.441, \eta_{\mathrm{p}}^{2}=.002 ;$ interaction between participant gender and Location, $F(1,294)=.04, p=.834, \eta_{\mathrm{p}}^{2}<.001$.

Alibi accuracy. No significant effects were found for ratings of alibi accuracy: participant gender, $F(1,259)=1.69, p=.194, \eta_{\mathrm{p}}^{2}=.006$; Location, $F(1,259)=1.36, p=.245$, $\eta_{\mathrm{p}}^{2}=.005$; interaction between participant gender and Location, $F(1,259)=.42, p=.517, \eta_{\mathrm{p}}^{2}=$ .002 .

Confidence in alibi accuracy. Again, as with earlier analyses, no significant differences were found for participants' ratings of their confidence in their judgments about alibi accuracy: participant gender, $F(1,259)=.12, p=.731, \eta_{\mathrm{p}}^{2}<.001$; Location, $F(1,259)=.03, p=.875, \eta_{\mathrm{p}}^{2}<$ .001 ; interaction between participant gender and Location, $F(1,259)=.94, p=.334, \eta_{\mathrm{p}}^{2}=.004$.

Strength of evidence against the suspect. The evidence against the suspect was rated as significantly stronger at Ryerson $(M=3.54, S D=1.56)$ than at Iowa State $(M=3.00, S D=1.36)$, $F(1,293)=9.02, p=.003, \eta_{\mathrm{p}}^{2}=.060$. The main effect for participant gender, $F(1,293)=.26, p=$ $.608, \eta_{\mathrm{p}}^{2}=.001$, and the interaction between participant gender and Location, $F(1,293)=.98, p$ $=.323, \eta_{\mathrm{p}}^{2}=.003$, were not statistically significant.

Likelihood of suspect guilt. As with ratings for the strength of the evidence against the suspect, participants' estimates of the likelihood of suspect guilt were affected by Location only, with higher estimates from participants at Ryerson $(M=3.89, S D=1.47)$ than from participants at Iowa State $(M=3.38, S D=1.36), F(1,294)=10.54, p=.001, \eta_{\mathrm{p}}^{2}=.035$. No significant differences were found for the main effect of participant gender, $F(1,294)=.20, p=.655, \eta_{\mathrm{p}}^{2}=$ .001 , or for the interaction between Location and participant gender, $F(1,294)=1.35, p=.246$, $\eta_{\mathrm{p}}^{2}=.005$

Verdict choice. A loglinear analysis using backward elimination was computed using 
sample Location, participant gender, and Verdict Choice. The goodness-of-fit test of the likelihood ratio of the final model with unnecessary terms removed is not significant $\chi^{2}(2)=$ $3.57, p=.168$, suggesting that the model is a good fit for the data. In the final model of the analysis, the generating class includes two terms: the interaction between Location and participant gender, and the interaction between Location and Verdict Choice. This first interaction is to be expected, as the proportion of women was notably higher in the sample from Ryerson than it was in the sample from Iowa State. The appropriate follow-up analysis to probe the second interaction was conducted earlier (see Verdict Choice section of the analyses comparing Alibi and No-Alibi Control conditions). Briefly, participants at Ryerson provided more guilty verdicts $(33.3 \%)$ than did participants at Iowa State $(22.5 \%)$. Regardless, participant gender was not included in any terms in the final model, suggesting that it is unrelated to verdict choice.

Willingness to sign the petition. A loglinear analysis using backward elimination was computed using Location, participant gender, and Verdict Choice. The goodness-of-fit test of the likelihood ration of the final model was not significant, $\chi^{2}(4)=1.55, p=.818$, suggesting that the model is a good fit for the data. After all unnecessary terms were removed from the model, the only term remaining was an interaction between Location and participant gender. As noted in the previous paragraph, this interaction indicates only that there is a greater proportion of female participants in the sample from Ryerson than there is in the sample from Iowa State. As no terms that included willingness to sign the petition were included in the final model, the results suggest that Location and participant gender were not related to participants' decision whether to sign the petition. 


\section{Appendix K: Psychometric Analysis of the ACT for Experiment 1}

When all items were included, the scale achieved very good internal consistency $(\alpha=$ .852). Analysis of the individual items shows that removing one item ("strong, tough government will harm not help our country" - reverse scored) would slightly increase the alpha $(\alpha=.853)$. Additionally, removing two other items ("It's great that many young people today are prepared to defy authority" - reverse scored; "Nobody should stick to the 'straight and narrow.' Instead people should break loose and try out lots of different ideas and experiences" - reverse scored) would not lower the alpha level. These three items correlate poorly with the other items on the measure $(r$ 's $<.3)$, but item-total correlations for the whole scale may be somewhat low in a measure with three subscales. However, these three items were flagged as potentially problematic.

As expected, the alphas for the individual subscales were lower than that of the total scale, because of the reduction in the number of items included for analysis. The Conservatism subscale achieved acceptable internal consistency $(\alpha=.747, N=294)$. On this subscale, deleting any items would produce a notable reduction in the alpha, though for the item about defying authority, the reduction would be small $(\alpha=.744)$. Although the item-total correlation for this item was adequate $(r=.361)$, it only correlated well with one other item. The other inter-item correlations are acceptable. Overall, it seems that the item regarding young people defying authority should be removed. The Traditionalism subscale showed good internal consistency $(\alpha=$ $.797, N=287$ ), though removing the item about breaking out of the "straight and narrow" would lead to a higher alpha $(\alpha=.819)$. The item-total correlation for this item is low $(r=.262)$ and it correlates poorly with all other items on the subscale (all $r<.243$ ). Given its poor results, this item should be removed from the analyses. Authoritarianism showed somewhat low internal 
consistency $(\alpha=.679, N=297)$. Alpha would be increased $(\alpha=.700)$ if the item about strong, tough government was removed. Its item-total correlation is low $(r=.224)$ and it only correlates well $(r=.376)$ with one other item on the subscale (all other $r<.178$ ). Consequently, it should also be removed.

When a reliability analysis was computed on the total scores, but with these three items removed, the scale still achieved very good internal consistency $(\alpha=.854, N=288)$. Deleting any items would reduce the alpha, and the item-total correlations were within acceptable limits (all $r>$.370). All retained items correlate well with at least some other items, particularly those belonging to the same subscale. Consequently, the total scores for the overall scale and for the subscales were computed on a 15-item ACT, rather than on the original 18-item version.

Participants who did not respond to particular items were not assigned scores where the item was used to calculate a total score. In other words, if a participant did not answer one question on the Conservatism subscale, s/he would be assigned a score for the Traditionalism and Authoritarianism subscales, but not for the Conservatism subscale and not for the whole scale.

The possible total scores of the ACT ranged from 15 to 105 . The mean slightly lower than the midpoint $(M=55.74, S D=13.78, N=288)$, and observed scores ranged from 23 to 98 . On the subscales, possible scores ranged from 5 to 35 . For Conservatism, the mean score was $18.19(S D=5.47, N=296$, range: $5-32)$; for Traditionalism, the mean score was $17.10(S D=$ $6.90, N=290$, range: 5-34), and for Authoritarianism, the mean was $20.42(S D=4.76, N=297$, range: 5-35). Distributions of scores for the total scale and for each of the subscales were approximately normal. Unlike with the Right-Wing Authoritarianism scale used by Cowan (2012), some participants did receive high scores on this measure.

Scores on the Authoritarianism subscale, $t(294.95)=.86, p=.389, d=0.10$, and the ACT 
total scores, $t(286)=-.17, p=.865, d=-0.02$, did not vary between the Iowa State and Ryerson samples. Participants at Iowa State $(M=18.94, S D=5.535)$ had significantly higher scores than participants at Ryerson $(M=17.56, S D=5.52)$ on the Conservatism subscale, $t(294)=2.17, p=$ $.031, d=0.25$. Participants at Iowa State $(M=15.98, S D=6.28)$ also had significantly lower scores than participants at Ryerson $(M=18.06, S D=7.27)$ on the Traditionalism subscale, $t(288)$ $=-2.58, p=.010, d=-0.39$. 


\section{Appendix L: Codebook for Qualitative Data; All Experiments}

\section{A Priori Codes}

1. Accurate - alibi is a narrative about where you were at the time of a crime/incident (implying that you could not be the perpetrator).

2. Alibi is a narrative about where the person was at a particular time (but does NOT make reference to a criminal defense).

3. Excuse - do they use the word "excuse"? Imply this somehow? An explanation?

a. "a plead to get out of something"

4. Time period - is there confusion about what time period is included in an alibi?

5. Alibi refers to the evidence rather than the narrative. E.g., your alibi is the people who can say you were somewhere.

6. Veracity - alibi could be true or false, its accuracy will be investigated/tested/evaluated, people may misremember

7. Reference to use in criminal proceedings/defense, related to a crime (but does NOT discuss narratives)

\section{Codes Added Based on Content of Experiment 1 Responses}

8. Alibi is always a lie/cover-up; there is an implication of someone lying to cover up their involvement in a crime. With this, the person is guilty and trying to divert suspicion. Could refer to accomplices in a crime.

9. Inaccurate, no useful information

a. Includes 'I don't know"

b. These aren't even close

c. May be too vague to be certain what the participant is referring to

10. Any evidence for innocence

a. E.g. ("evidence that supports the person is not guilty")

b. Difference between this and \#5 is that this is vague, much more open. 5 is more specific to evidence that you were somewhere else.

11. Alibi should have some kind of evidence to back it up, to be a real alibi, it must be confirmed (they MUST make primary reference to a narrative; can pretty much only occur in conjunction with a 1 or 2 )

\section{Code Added Based on Content of Experiment 3 Responses}

12. Latin for "elsewhere." 


\section{Appendix M: Inferential Tests Based on Qualitative Responses for Experiment 1}

It is possible that participants' different understandings of what an alibi is affected their judgments about the legal aspects of the case. Differences in responses between participants who understood that an alibi referred to a narrative regarding where a suspect was during the time of a crime and participants whose responses emphasized evidence to support a narrative were investigated (see Table M1 for statistical results of continuous outcome variables). None of the independent-samples $t$-tests showed significant differences between groups. As well, Verdict Choice, $\chi^{2}(1)=.16, p=.737, \varphi=-.03$, and participants' petition signing behaviour, $\chi^{2}(1)=.67, p$ $=.428, \varphi=.06$, were not significantly related to their understanding of the term "alibi."

\section{Table M1}

Comparison of legal judgments between participants who provided correct definitions and participants who emphasized an alibi as evidence.

\begin{tabular}{lcccccc}
\hline Outcome & $d f$ & $t$ & $p$ & $d$ & $M 1(S D)$ & $M 2(S D)$ \\
\hline Statement honesty & 184 & .50 & .620 & 0.08 & $4.47(1.41)$ & $4.36(1.48)$ \\
Confidence in statement honesty & 184 & -.07 & .948 & -0.01 & $4.94(1.41)$ & $4.96(1.40)$ \\
Accuracy & 167 & -.37 & .712 & -0.07 & $4.47(1.43)$ & $4.56(1.52)$ \\
Confidence in accuracy & 167 & -1.48 & .140 & -0.24 & $4.93(1.29)$ & $5.24(1.26)$ \\
Strength of evidence against the & 184 & .84 & .405 & 0.13 & $3.40(1.44)$ & $3.22(1.47)$ \\
$\quad$ Luspect & & & & & & \\
Likelihood of guilt & 184 & .68 & .500 & 0.11 & $3.73(1.48)$ & $3.58(1.47)$ \\
\hline
\end{tabular}

Note. M1 refers to participants who referred to the alibi as a narrative regarding a suspect's whereabouts at the time of a crime, whereas M2 refers to the participants whose responses emphasized the evidence supporting a statement. 


\section{Appendix N: Experiment 1 Debrief Forms}

\section{Debriefing - Criminal Investigation 1}

The study that you just participated in examines the factors associated with the evaluation of an alibi. An alibi is the explanation a suspect gives to the police regarding his or her whereabouts when a crime is committed. The Innocence Project (http://www.innocenceproject.org/), an organization that helps the wrongly convicted, a found that in approximately $25 \%$ of their DNA exoneration cases, the defendant presented an alibi that wasn't believed. Previous research has demonstrated that alibi evidence (that is ultimately true) is often considered 'weak' by the justice system and therefore ignored (Burke, Turtle and Olson, 2007). A lot of studies have focused on the strength of corroborating evidence to explain why some alibis are believed, while others are not. For example, an alibi that is confirmed by a stranger's testimony and physical evidence that would be hard to fabricate will be believed more often than an alibi corroborated by a relative of the suspect with no physical evidence to back it up. In this study, we were interested in how the content of the alibi influenced whether the alibi was believed and what people thought was the probability that the suspect was guilty. Essentially, we wanted to know whether the type of activities described in the alibi or the strength of the evidence supporting the alibi influenced belief in the alibi and decisions about the suspect's guilt. The moral desirability of the actions that the suspect described and the strength of the physical evidence supporting the alibi (independent variables) were manipulated. The suspect might be describing doing something morally desirable, morally undesirable, or morally neutral. Also, there could be strong, weak, or no physical evidence to support the suspect's alibi. All of the participants in this study read the same crime information, but the alibi and corroborating evidence offered by the suspect was different.

Our first hypothesis was that morally undesirable alibis would be rated as more believable and the suspect would be judged to be less likely to be guilty than with neutral alibis. We expected that participants would interpret these alibis as evidence of honesty - why would a suspect admit to something that makes him look bad unless it was the truth? We also expected that participants would be more likely to agree to sign the petition supporting the suspect if the alibi was morally undesirable, because they thought the alibi was true and the suspect was innocent.

Our second hypothesis was that alibis supported by strong evidence would be rated as more believable and the suspect would be judged as less likely to be guilty than alibis supported by weak or no physical evidence. We expected that participants would be more likely to sign the petition if there was strong evidence supporting the alibi.

Our third hypothesis was that neutral or morally desirable alibis would need stronger corroborating evidence to be believable than morally undesirable alibis.

The results will allow us to see if the content of suspects' alibis has an influence on juror decisions of guilt and alibi believability and their petition-signing behaviour (the dependent variables). This information may allow us to help the justice system evaluate and use alibi information more appropriately. 
If you know of others who will be participating in this experiment, please refrain from discussing it with them. We do not want our future participants to be aware of the procedures and expected findings.

Thank you for participating in our research. If you have any questions about this study, please contact:

$\begin{array}{ll}\text { Sara Cowan, MA } & \text { Christian A. Meissner, Ph.D. } \\ \text { scowan@iastate.edu } & \text { cameissn@iastate.edu } \\ \text { sara.cowan@psych.ryerson.ca } & \end{array}$




\section{Debriefing - Criminal Investigation 1}

The study that you just participated in examines the factors associated with the evaluation of an alibi. An alibi is the explanation a suspect gives to the police regarding his or her whereabouts when a crime is committed. The Innocence Project (http://www.innocenceproject.org/), an organization that helps the wrongly convicted, has found that in approximately $25 \%$ of their DNA exoneration cases, the defendant presented an alibi that wasn't believed. Previous research has demonstrated that alibi evidence (that is ultimately true) is often considered 'weak' by the justice system and therefore ignored (Burke, Turtle and Olson, 2007). A lot of studies have focused on the strength of corroborating evidence to explain why some alibis are believed, while others are not. For example, an alibi that is confirmed by a stranger's testimony and physical evidence that would be hard to fabricate will be believed more often than an alibi corroborated by a relative of the suspect with no physical evidence to back it up. In this study, we were interested in how the content of the alibi influenced whether the alibi was believed and what people thought was the probability that the suspect was guilty. Essentially, we wanted to know whether the type of activities described in the alibi or the strength of the evidence supporting the alibi influenced belief in the alibi and decisions about the suspect's guilt. The moral desirability of the actions that the suspect described and the strength of the physical evidence supporting the alibi (independent variables) were manipulated. The suspect might be describing doing something morally desirable, morally undesirable, or morally neutral. Also, there could be strong, weak, or no physical evidence to support the suspect's alibi. All of the participants in this study read the same crime information, but the alibi and corroborating evidence offered by the suspect was different.

Our first hypothesis was that morally undesirable alibis would be rated as more believable and the suspect would be judged to be less likely to be guilty than with neutral alibis. We expected that participants would interpret these alibis as evidence of honesty - why would a suspect admit to something that makes him look bad unless it was the truth? We also expected that participants would be more likely to agree to sign the petition supporting the suspect if the alibi was morally undesirable, because they thought the alibi was true and the suspect was innocent.

Our second hypothesis was that alibis supported by strong evidence would be rated as more believable and the suspect would be judged as less likely to be guilty than alibis supported by weak or no physical evidence. We expected that participants would be more likely to sign the petition if there was strong evidence supporting the alibi.

Our third hypothesis was that neutral or morally desirable alibis would need stronger corroborating evidence to be believable than morally undesirable alibis.

The results will allow us to see if the content of suspects' alibis has an influence on juror decisions of guilt and alibi believability and their petition-signing behaviour (the dependent variables). This information may allow us to help the justice system evaluate and use alibi information more appropriately.

If you know of others who will be participating in this experiment, please refrain from discussing it with them. We do not want our future participants to be aware of the procedures and expected 
findings.

Thank you for participating in our research. If you have any questions about this study, please contact:

Sara Cowan, MA

sara.cowan@psych.ryerson.ca

416-979-5000 x2190
Tara Burke, Ph.D.

tburke@psych.ryerson.ca

416-979-5000 x6519

If you any have questions about receiving your Psychology 102/202 credit for participation please contact: thepool@psych.ryerson.ca 


\section{Appendix O: Experiment 2 Consent Form}

RYERSON

UNIVERSITY

\author{
Ryerson University \\ Consent Agreement \\ Criminal Investigation 2
}

You are being asked to participate in a research study. Before you give your consent, it is important that you read the following information and ask as many questions as necessary to be sure you understand what you will be asked to do.

Investigator:
Supervisor: Dr. Tara Burke

Associate Professor

Department of Psychology

Purpose of the Study: The purpose of this study is to examine how individuals determine the guilt or innocence of a suspect in a crime. In order to study this issue, approximately 300 students will be asked to participate in this research. All Ryerson students enrolled in Psy102 or Psy202 are eligible to participate.

Description of the Study: If you decide to participate in this research, you will be asked to do the following: You will read some information about a hypothetical criminal case, read a transcript of a police interview with a suspect, and answer some questions about your thoughts about the case. This study will take place in the Psychology Research and Training Centre at 105 Bond Street. It will take approximately 60 minutes to complete.

What is Experimental in this Study: None of the procedures or questionnaires used in this study are experimental in nature, in the sense that they have all been used by other researchers and found to be useful procedures and questionnaires. From a technical or procedural point of view, part of this study is considered "experimental," because by following the procedure described above, the study examines the impact of one variable (called the "independent variable") on another variable ("called the dependent variable"). You will be given more information about the independent and dependent variables in this study at the end of today's session.

Risks or Discomforts: Occasionally people feel uncomfortable when answering questions that ask about attitudes toward controversial social issues (e.g., vaccination). While we do not anticipate that any of the questions you will be responding to are controversial, if any aspect of this study makes you feel uncomfortable, you may choose not to answer certain questions, or to withdraw from the study at any time without penalty.

Benefits of the Study: We anticipate that you will benefit from this study by learning about how psychology research is conducted. When the session is over, we will describe the purpose and hypotheses of the study to you in more detail. Also, once we have analysed the data (toward the end of the term), you are welcome to contact the researchers by e-mail to view the results.

Confidentiality: Your responses in this research will be confidential. Your name may be linked 
to your responses. As such, your responses are not anonymous. Any materials that include your name - including this consent form - will be stored confidentially for a minimum of 5 years. Physical materials will be stored in a locking filing cabinet in a locked room, and computer files will be stored on password-protected computers. Only the investigator and her faculty supervisor will have access to this information. Wherever possible, questionnaires will be identified only by a participant number assigned to you by the researchers. This number will not be used later to identify you and is unrelated to your Sona ID. This consent form (if you decide to participate) will be filed separately from any data collected in the experiment. The online questionnaire is hosted by Qualtrics, a web survey company located in the USA and as such, is subject to U.S. laws; in particular, the US Patriot Act, which allows authorities access to the records of internet service providers. This survey or questionnaire does not ask for personal identifiers or any information that may be used to identify you. However, if you choose to participate in the study, you understand that your responses to the survey questions will be stored, and can be accessed, in the USA. The security and privacy policy for the web survey company can be found at the following link: http://www.qualtrics.com/security-statement/

Incentives to Participate: Although we appreciate the contributions of participants in our research, you will not be paid for your participation in this study. However, you will receive $1 \%$ course credit for your Introductory Psychology course. If you would prefer to walk through the study - that is, if you would like to observe the research process but not fill out any questionnaires or provide any personal data - you will still be given $1 \%$ course credit. Note that while you can take part in as many psychology research studies as you wish, there is a limit in the course credit you can earn in any one term. This limit is in your syllabus.

Voluntary Nature of Participation: Participation in this study is voluntary. If you decide to participate, you are free to withdraw your consent and to stop your participation at any time without penalty or loss of benefits to which you are allowed. Should you withdraw from the study, you will still be given your $1 \%$ course credit (provided you have not already received the maximum allotted for research participation).

Dissemination of Results: The results of this study will form part of Sara Cowan's PhD dissertation. As well, results may be shared with others at scholarly meetings or as part of published papers. However, all information will be presented in aggregate form. That is, none of your individual information will be identifiable in any way. Anonymized data may be provided to other researchers for the purpose of study or verification of results; any data that is shared will NOT include the names of ANY participants.

Questions about the Study: If you have any questions about the research now, please ask. If you have questions later about the research, you may contact: Sara Cowan 416-979-5000 x 2190, sara.cowan@psych.ryerson.ca ; Dr. Tara Burke, 416-979-5000, ex. 6519, tburke@psych.ryerson.ca

\section{If you any have questions about receiving your Psychology 102/202 credit for participation please contact: thepool@ psych.ryerson.ca}

If you have questions regarding your rights as a human subject and participant in this study, you 
may contact the Ryerson University Research Ethics Board for information: Lynn Lavallée, PhD., Research Ethics Board, c/o Office of the Vice President, Research and Innovation, Ryerson University, 350 Victoria Street, Toronto, ON M5B 2K3, 416-979-5042, rebchair@ryerson.ca

Agreement: Your signature below indicates that you have read the information in this agreement and have had a chance to ask any questions you have about the study. Your signature also indicates that you agree to be in the study and have been told that you can change your mind and withdraw your consent to participate at any time.

You have been told that by signing this consent agreement you are not giving up any of your legal rights.

Name of Participant (please print)

Signature of Participant

Date

Signature of Investigator

Date 


\section{Appendix P: Experiment 2 Reconsent Form \\ Reconsent - Criminal Investigation 2}

Agreement: Your signature below indicates that you have read the information in this debriefing form and have had a chance to ask any questions you have about the study. Your signature also indicates that you agree to allow the experimenter to retain the data collected in the study now that you have full knowledge of the purpose of this study. You have been given a copy of this agreement.

You have been told that by signing this consent agreement, you are not giving up any of your legal rights.

\section{Master List (CHECK ONE BOX)}

The researcher CAN include my name on the Master List The researcher CANNOT include my name on the Master List

Questionnaire Responses (CHECK ONE BOX)

The researcher CAN analyze my questionnaire responses The researcher CANNOT analyze my questionnaire responses 


\section{Appendix Q: Experiment 2 Sona Recruitment Information}

\section{Study Name:}

Criminal Investigation 2

Brief Abstract: We are exploring how people determine the guilt or innocence of a suspect. You will be given information about a police investigation and be asked to give your impressions about the case. This Social Psychology study involves one 60 minute lab visit.

\section{Detailed Description:}

Please DO NOT sign up for this study if you have already participated in the Criminal Investigations 1 study, or the Criminal Investigations 2 study in a previous term. The study will take place at the South Bond Building (105 Bond), 2nd Floor. You can take the stairs or the elevator to the second floor and have a seat in the lobby. Someone will meet you there. If need be, the lab phone extension is $\times 2190$. Approximately 200-250 participants will be recruited. All participants will be students at Ryerson University currently enrolled in the Introductory Psychology course PSY102 or PSY202. The study will consist of one 60 minute session which may be completed in a group setting, although all data collection is individual. You will be asked to read a short summary of a criminal case and then provide feedback about your impressions of the case and the individual involved. You will then be asked to complete a questionnaire designed to measure beliefs and attitudes relevant to the legal context. While no risks are anticipated, it is possible that you may feel slight discomfort in answering some questions relating to attitudes, beliefs, or personal opinions. However, all measures used in this research have been used in previous research and are not believed to cover any particularly controversial topics. You are free not to answer any and all questions you feel uncomfortable answering. By participating in this study, you may benefit from a greater understanding of how research is conducted in the field of Social Psychology. 


\section{Appendix R: Experiment 2 Case Materials}

\section{Police Summary Report: Seattle Condition}

$\begin{array}{ll}\text { Supplemental } & \text { Seattle Police Division } \\ \text { Crime Report } & \text { Form } 38.3 \text { Rev. 5/09 }\end{array}$

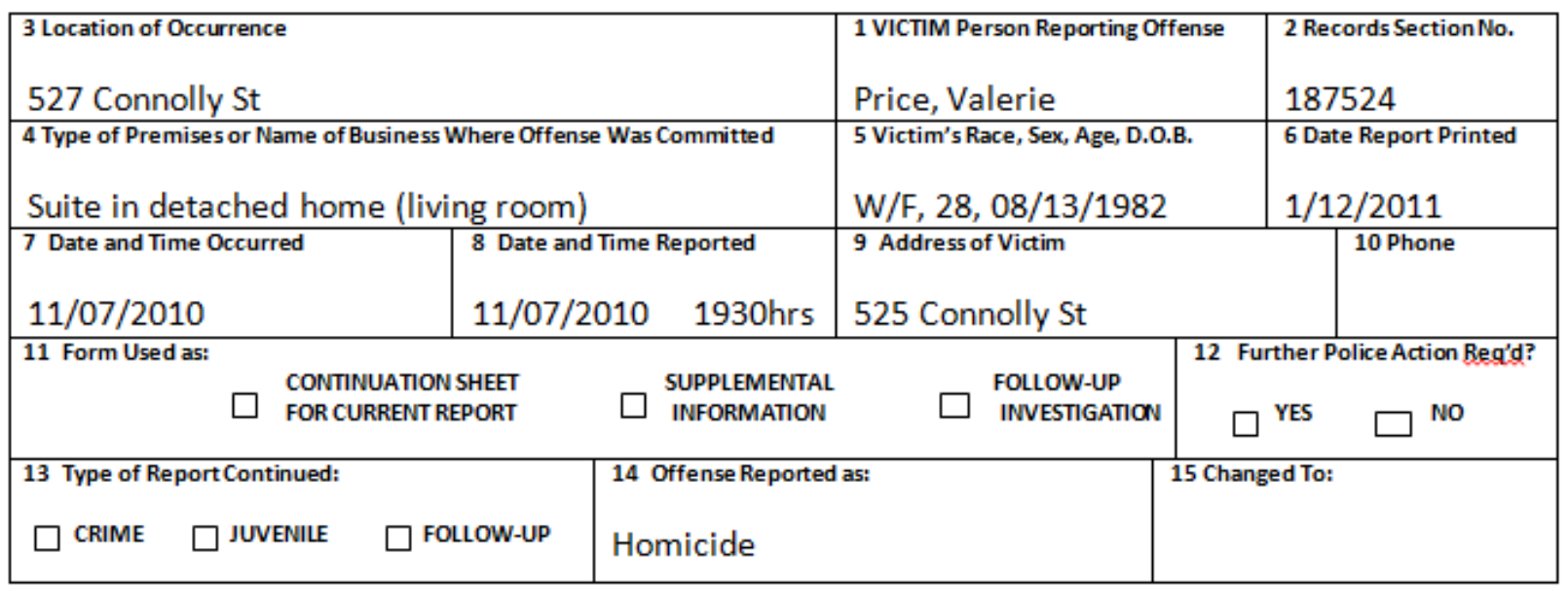

\section{NARRATIVE:}

On Sunday November 7, 2010, at approximately 1915 hours, a 911 distress call was received from Janice Parker of 525 Connelly St, reporting a scream and gunshots from the next door residence (527 Connelly St.) and an unidentified male fleeing the residence. Dispatch contacted Officers Fisher and Balfour (11 Division), and dispatched them to the scene. After entering and securing the premises, Fisher called 11 Division to report an apparent homicide at 527 Connelly St. The victim was found on the floor of the living room and it appeared that she had been shot twice in the chest. EMT personnel confirmed the victim was deceased. The investigation was then assigned to Detective Bailey, who prior to leaving Southwest Precinct, contacted Coroner's Investigator, T. Stanfield and Evidence Technician, B. Alexander.

Upon arrival at 527 Connolly St at approximately 2000 hours, Detective Bailey was met by Officer Fisher. Officer Fisher stated that he and Officer Balfour were dispatched to the scene in response to a 911 call. When the officers first arrived, they were met by personnel from No. 6 fire pumper and life squad No.2. The officers were told that the victim showed no signs of life and the death appeared to be a homicide. They were also told that the firefighters first found the victim lying face down on the living room floor. The victim, later identified as Valerie Price, was then turned over onto her back by Fire Department personnel as they attempted to render aid. Nothing else in the apartment was touched by Fire Department personnel. 
Officer Fisher also stated that after he viewed the body and carefully checked the other rooms in the apartment, the scene was secured and protected. Officer Fisher then contacted

Southwest Precinct for assistance. While waiting for the arrival of the detectives, Officer Fisher interviewed Janice Parker, the neighbour who placed the 911 call in the adjacent home at 525 Connelly St. During this time, Officer Balfour remained at the crime scene and ensured that nothing was disturbed. In the interview with Officer Fisher, Ms. Parker reported hearing a scream from the residence, followed by 2 or 3 gunshots. When she looked outside her window, she saw a male running down the street, wearing blue jeans, a green jacket with two reflective stripes down each arm, and a black baseball cap with a picture or logo on the front. The male was approximately 5'10" to 6'1" tall, average build, with no facial hair, between 30 to 40 years old. It was dusk, so she did not get a good look at the suspect and was not able to say for sure what race the man was.

\section{EVIDENCE:}

All evidence collected at the crime scene was collected by Evidence Technician Alexander.

\section{ADDITIONS:}

01/12/2011: In response to media appeals for information about the case, James Hearn (517 Taunton St) contacted $11^{\text {th }}$ District to report that the description of the culprit matched a nearby neighbor, Robert Harris (532 Taunton St). Officers Jiminez and Robertson were sent to conduct a preliminary interview with Harris. In the hallway of his home the officers observed a black baseball cap with an Arizona Cardinals logo and a green jacket with reflective stripes hanging on a peg. These items of clothing also match the description of the clothing worn by the culprit. The officers observed that Harris appeared nervous during the encounter, and believed that he was not forthcoming in his answers to their questions. As a result, officers determined Harris may be a person of interest and decided to take Mr. Harris in for secondary questioning with a Detective at the police station. 


\section{Police Summary Report: Vancouver Condition}

Supplemental Vancouver Police Department

Crime Report Form 38.3 Rev. 5/09

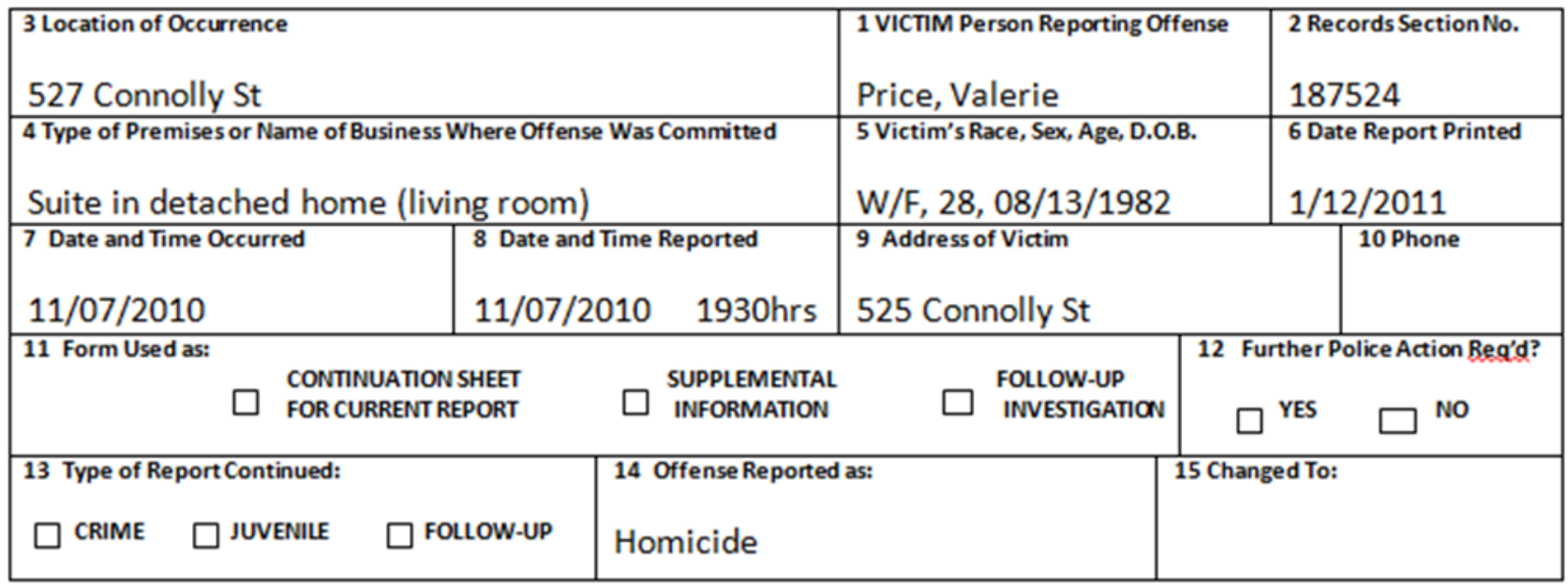

\section{NARRATIVE:}

On Sunday November 7, 2010, at approximately 1915 hours, a 911 distress call was received from Janice Parker of 525 Connelly St, reporting a scream and gunshots from the next door residence (527 Connelly St.) and an unidentified male fleeing the residence. Dispatch contacted Officers Fisher and Balfour (District 3), and dispatched them to the scene. After entering and securing the premises, Fisher called District 3 to report an apparent homicide at 527 Connelly St. The victim was found on the floor of the living room and it appeared that she had been shot twice in the chest. EMT personnel confirmed the victim was deceased. The investigation was then assigned to Detective Bailey, who prior to leaving District 3, contacted Coroner's Investigator, T. Stanfield and Evidence Technician, B. Alexander.

Upon arrival at 527 Connolly St at approximately 2000 hours, Detective Bailey was met by Officer Fisher. Officer Fisher stated that he and Officer Balfour were dispatched to the scene in response to a 911 call. When the officers first arrived, they were met by personnel from No. 6 fire pumper and life squad No.2. The officers were told that the victim showed no signs of life and the death appeared to be a homicide. They were also told that the firefighters first found the victim lying face down on the living room floor. The victim, later identified as Valerie Price, was then turned over onto her back by Fire Department personnel as they attempted to render aid. Nothing else in the apartment was touched by Fire Department personnel.

Officer Fisher also stated that after he viewed the body and carefully checked the other rooms 
in the apartment, the scene was secured and protected. Officer Fisher then contacted Southwest Precinct for assistance. While waiting for the arrival of the detectives, Officer Fisher interviewed Janice Parker, the neighbour who placed the 911 call in the adjacent home at 525 Connelly St. During this time, Officer Balfour remained at the crime scene and ensured that nothing was disturbed. In the interview with Officer Fisher, Ms. Parker reported hearing a scream from the residence, followed by 2 or 3 gunshots. When she looked outside her window, she saw a male running down the street, wearing blue jeans, a green jacket with two reflective stripes down each arm, and a black baseball cap with a picture or logo on the front. The male was approximately 5'10" to 6'1" tall, average build, with no facial hair, between 30 to 40 years old. It was dusk, so she did not get a good look at the suspect and was not able to say for sure what race the man was.

\section{EVIDENCE:}

All evidence collected at the crime scene was collected by Evidence Technician Alexander.

\section{ADDITIONS:}

01/12/2011: In response to media appeals for information about the case, James Hearn (517 Taunton St) contacted Division 3 to report that the description of the culprit matched a nearby neighbor, Robert Harris (532 Taunton St). Officers Jiminez and Robertson were sent to conduct a preliminary interview with Harris. In the hallway of his home the officers observed a black baseball cap with an Arizona Cardinals logo and a green jacket with reflective stripes hanging on a peg. These items of clothing also match the description of the clothing worn by the culprit. The officers observed that Harris appeared nervous during the encounter, and believed that he was not forthcoming in his answers to their questions. As a result, officers determined Harris may be a person of interest and decided to take Mr. Harris in for secondary questioning with a Detective at the police station. 
Suspect Information Sheets: Seattle Condition

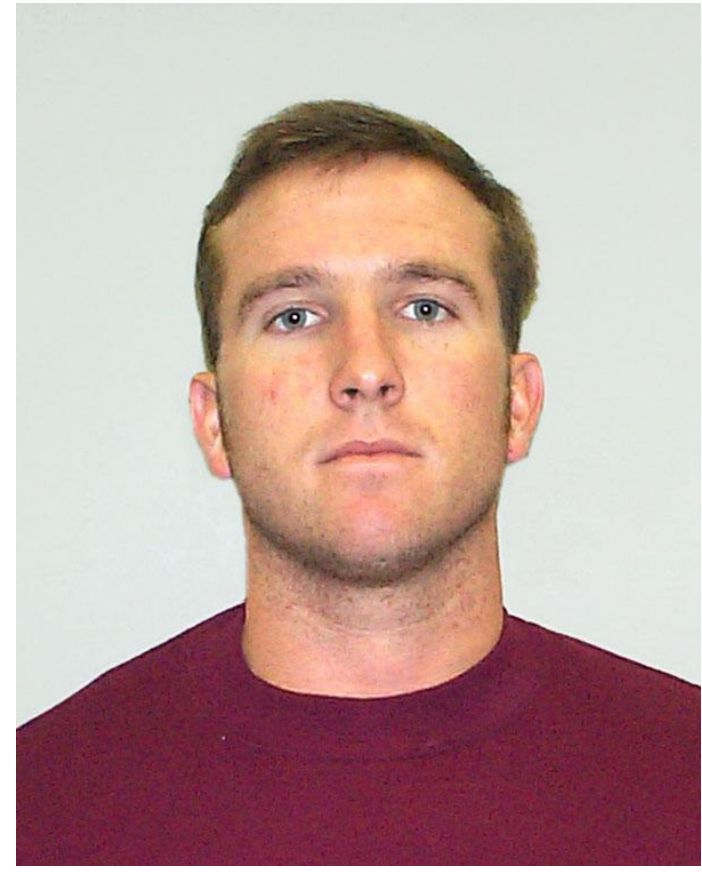

\section{SUSPECT}

NAME: Harris, Robert Michael

DATE OF BIRTH: 10/27/1981

SEX: Male

NATIONALITY: Canadian (Naturalized US Citizen)

RACE: Caucasian (White)

ADDRESS: 532 Taunton St.

MARITAL STATUS: Single

PREVIOUS CRIMINAL RECORD: NO 


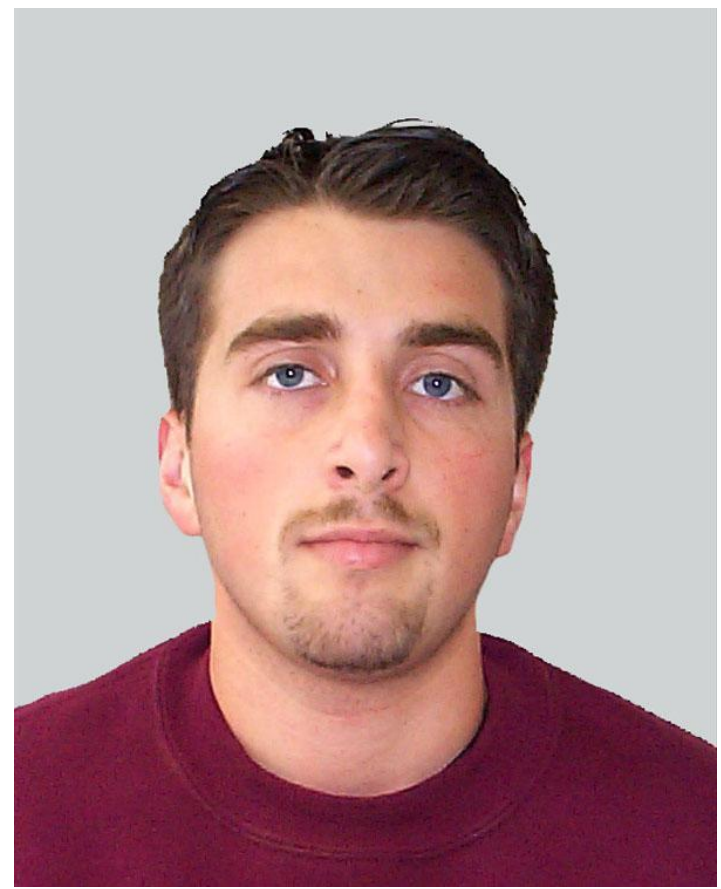

\section{SUSPECT}

NAME: Harris, Robert Michael

DATE OF BIRTH: 10/27/1981

SEX: Male

NATIONALITY: Canadian (Naturalized US Citizen)

RACE: Caucasian (White)

ADDRESS: 532 Taunton St.

MARITAL STATUS: Single

PREVIOUS CRIMINAL RECORD: NO 


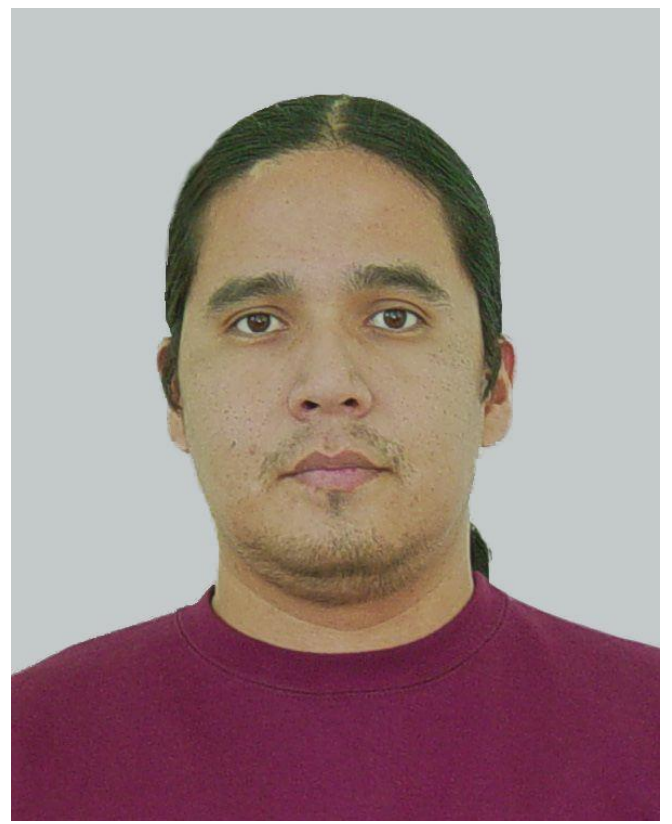

\section{SUSPECT}

NAME: Harris, Robert Michael

DATE OF BIRTH: $10 / 27 / 1981$

SEX: Male

NATIONALITY: Canadian (Naturalized US Citizen)

RACE: First Nations/Aboriginal

ADDRESS: 532 Taunton St.

MARITAL STATUS: Single

PREVIOUS CRIMINAL RECORD: NO 


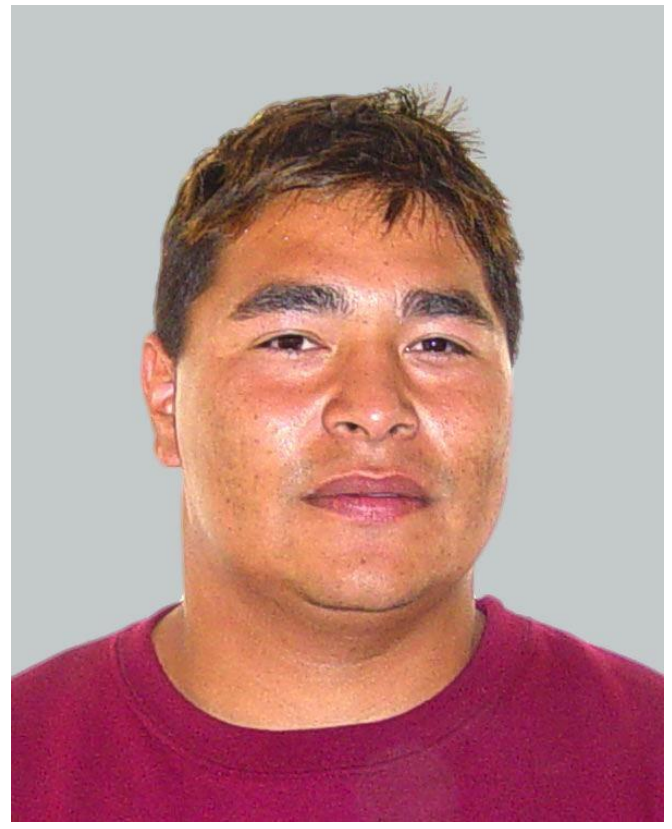

\section{SUSPECT}

NAME: Harris, Robert Michael

DATE OF BIRTH: $10 / 27 / 1981$

SEX: Male

NATIONALITY: Canadian (Naturalized US Citizen)

RACE: First Nations/Aboriginal

ADDRESS: 532 Taunton St.

MARITAL STATUS: Single

PREVIOUS CRIMINAL RECORD: NO 
Suspect Information Sheets: Vancouver

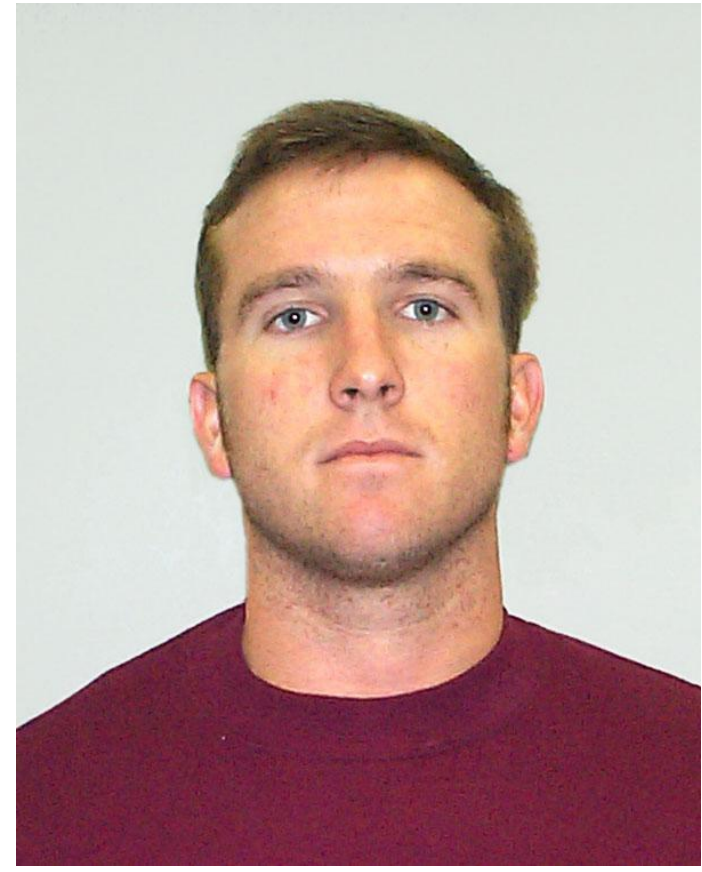

\section{SUSPECT}

NAME: Harris, Robert Michael

DATE OF BIRTH: 10/27/1981

SEX: Male

NATIONALITY: Canadian

RACE: Caucasian (White)

ADDRESS: 532 Taunton St.

MARITAL STATUS: Single

PREVIOUS CRIMINAL RECORD: NO 


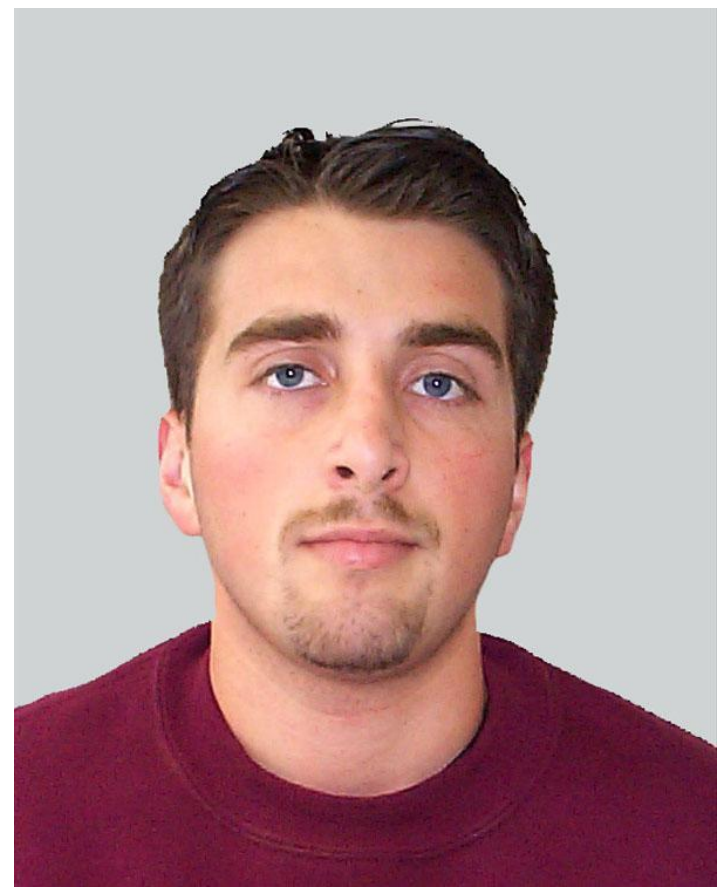

\section{SUSPECT}

NAME: Harris, Robert Michael

DATE OF BIRTH: 10/27/1981

SEX: Male

NATIONALITY: Canadian

RACE: Caucasian (White)

ADDRESS: 532 Taunton St.

MARITAL STATUS: Single

PREVIOUS CRIMINAL RECORD: NO 


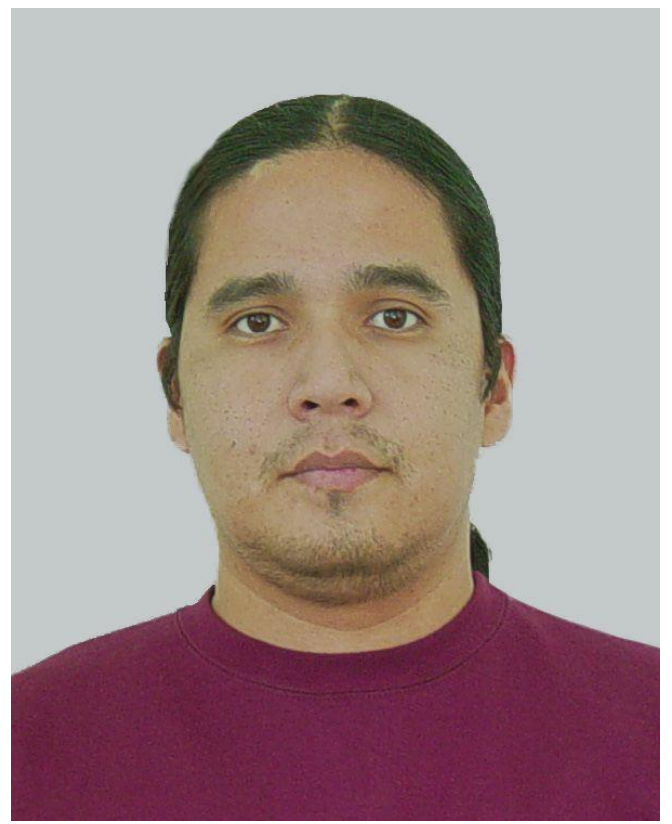

\section{SUSPECT}

NAME: Harris, Robert Michael

DATE OF BIRTH: $10 / 27 / 1981$

SEX: Male

NATIONALITY: Canadian

RACE: First Nations/Aboriginal

ADDRESS: 532 Taunton St.

MARITAL STATUS: Single

PREVIOUS CRIMINAL RECORD: NO 


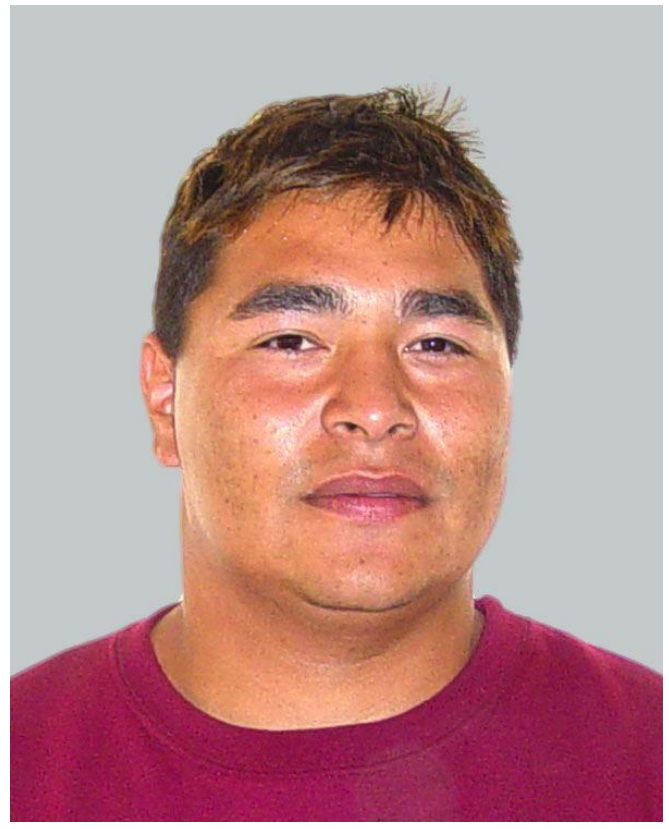

\section{SUSPECT}

NAME: Harris, Robert Michael

DATE OF BIRTH: $10 / 27 / 1981$

SEX: Male

NATIONALITY: Canadian

RACE: First Nations/Aboriginal

ADDRESS: 532 Taunton St.

MARITAL STATUS: Single

PREVIOUS CRIMINAL RECORD: NO 


\section{Police Interrogation Transcripts: Seattle Condition}

Supplemental Seattle Police Division

Crime Report Form 38.3 Rev. 5/09

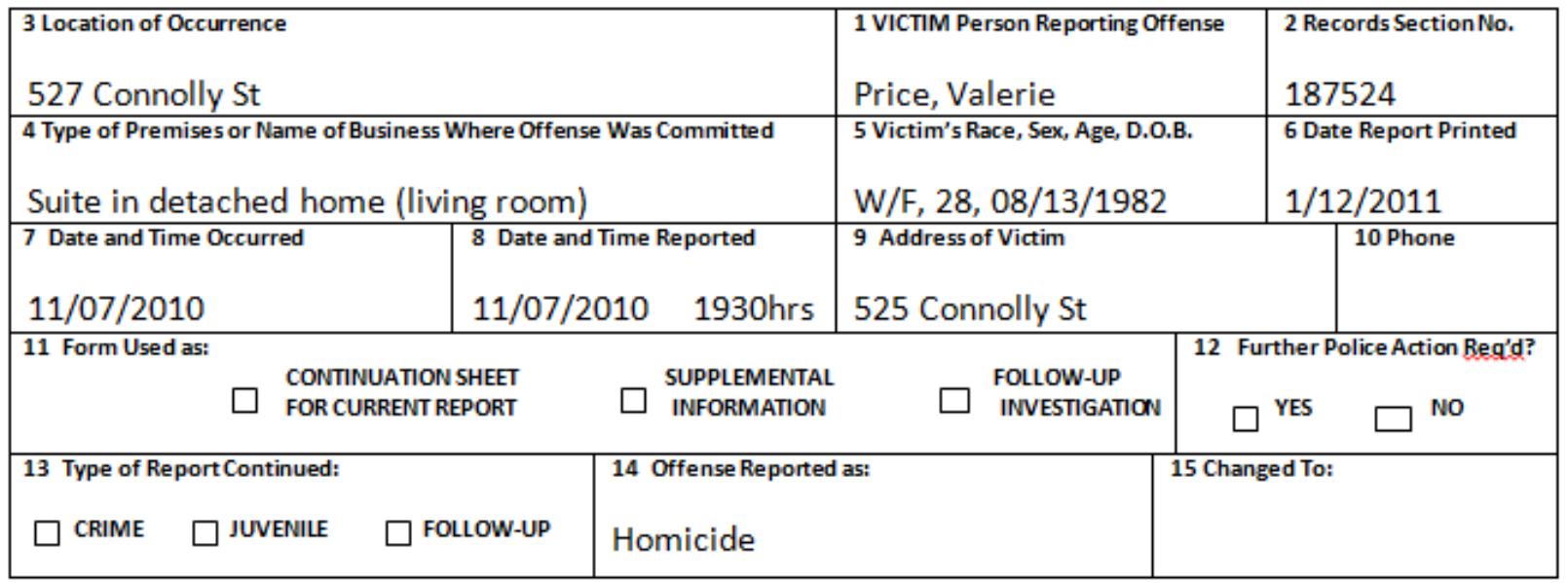

\section{INTERROGATION OF ROBERT HARRIS, 1/12/2011, 11:01PM:}

Det. Bailey: Could you state your full name for the records?

Harris: My name is Robert Harris.

Det. Bailey: Thank you. And where do you live, Mr. Harris?

Harris: 532 Taunton St.

Det. Bailey: And how long have you lived there?

Harris: About 5 years or so.

Det. Bailey: Good, thank you. We are investigating the murder of one of your neighbours,

Valerie Price, on November $7^{\text {th }}, 2010$, approximately two months ago. This is a photo of her

[shows Harris the photo]. Did you know the victim at all, Mr. Harris?

Harris: Yes. 
Det. Bailey: How well did you know her?

Harris: Not very. I mean, I'd recognize her, we'd say hi if we saw each other on the street, but that's about it.

Det. Bailey: How long had you known her?

Harris: I don't know, maybe a couple of years, but I'm really not sure.

Det. Bailey: And how would you characterize your relationship with her?

Harris: I don't know. Acquaintances, I guess.

Det. Bailey: And have you ever been inside her home?

Harris: No.

Det. Bailey: Could you detail for me, to the best of your ability, your whereabouts on November 7, 2010, the day of Ms. Price's murder two months ago. Start at the beginning of your day and continue until the end of that day. Keep in mind that we will be verifying your statement and any lies or omissions that we note will cast further suspicion on you. Murder is a serious crime, Mr. Harris, so really, it's in your best interest to be as honest and forthcoming as possible.

\section{VERSION 1 (Morally Undesirable)}

Harris: I got up between 8:30 and 9, spent the morning at home working on a project for work, watched the football game in the afternoon, then made dinner at home.

Det. Bailey: OK, were you still at home that same evening?

Harris: No.

Det. Bailey: Where were you that evening between 7 and 7:30PM, Mr. Harris?

Harris: I was at the home of my friend Michael Beauregard and his wife Kaitlynn. I think I got 
there at about twenty to seven, maybe quarter to.

Det. Bailey: Were they home?

Harris: Michael is away on a business trip, but Kaitlynn was there.

Det. Bailey: OK, we will be checking on that. What were you doing there?

Harris: That night I was in bed with Kaitlynn. We've been having an affair.

Det. Bailey: What time did you return home?

Harris: I think I left around 11:30 or so, and was home by 11:45.

Det. Bailey: And you're sure that is the time you returned home? It could not have been any earlier?

Harris: Yes, I'm positive.

Det. Bailey: Thank you. We're going to need you to provide us with a list of individuals who could support your statement and any physical evidence that could confirm what you say. After that, you are free to go, though we may need to interview you again.

END OF INTERVIEW

\section{VERSION 2 (Morally Neutral)}

Harris: I got up between 8:30 and 9, spent the morning at home working on a project for work, watched the football game in the afternoon, then made dinner at home.

Det. Bailey: OK, were you still at home that same evening?

Harris: No.

Det. Bailey: Where were you that evening between 7 and 7:30PM, Mr. Harris?

Harris: I was at the home of my friend Michael Beauregard and his wife Kaitlynn. I think I got 
there at about twenty to seven, maybe quarter to.

Det. Bailey: Were they home?

Harris: Michael is away on a business trip, but Kaitlynn was there.

Det. Bailey: OK, we will be checking on that. What were you doing there?

Harris: That night I was picking up some DVDs I let Michael and Kaitlynn borrow.

Det. Bailey: What time did you return home?

Harris: I think I left around 11:30 or so, and was home by 11:45.

Det. Bailey: And you're sure that is the time you returned home? It could not have been any earlier?

Harris: Yes, I'm positive.

Det. Bailey: Thank you. We're going to need you to provide us with a list of individuals who could support your statement and any physical evidence that could confirm what you say. After that, you are free to go, though we may need to interview you again.

END OF INTERVIEW

\section{VERSION 3 (Morally Desirable)}

Harris: I got up between 8:30 and 9, spent the morning at home working on a project for work, watched the football game in the afternoon, then made dinner at home.

Det. Bailey: OK, were you still at home that same evening?

Harris: No.

Det. Bailey: Where were you that evening between 7 and 7:30PM, Mr. Harris?

Harris: I was at the home of my friend Michael Beauregard and his wife Kaitlynn. I think I got 
there at about twenty to seven, maybe quarter to.

Det. Bailey: Were they home?

Harris: Michael is away on a business trip, but Kaitlynn was there.

Det. Bailey: OK, we will be checking on that. What were you doing there?

Harris: That night I was helping Michael and Kaitlynn move, loading boxes and furniture on the truck.

Det. Bailey: What time did you return home?

Harris: I think I left around 11:30 or so, and was home by 11:45.

Det. Bailey: And you're sure that is the time you returned home? It could not have been any earlier?

Harris: Yes, I'm positive.

Det. Bailey: Thank you. We're going to need you to provide us with a list of individuals who could support your statement and any physical evidence that could confirm what you say. After that, you are free to go, though we may need to interview you again. END OF INTERVIEW

\section{VERSION 4 (No-Alibi Control)}

Harris: Let me think. [PAUSE] That was about two months ago, so I'm having trouble remembering. What day of the week was that?

Det. Bailey: It was a Sunday.

Harris: In that case, if there was a football game on, I was probably watching that in the afternoon. 
Det. Bailey: Where were you that evening between 7 and 7:30PM, Mr. Harris?

Harris: Without checking my calendar, I really can't be sure. I just can't remember where I was that night.

Det. Bailey: Were you at home, or were you out that evening?

Harris: I just don't know. Some Sunday evenings I stay in, sometimes I go out.

Det. Bailey: So you do not have regular Sunday evening activities?

Harris: No. It varies.

Det Bailey: What kinds of activities might you do on a Sunday night?

Harris: Maybe a drink with friends, or a dinner date.

Det. Bailey: When you do go out on Sundays, what time do you usually return home?

Harris: It depends, usually not after 11:30 or so.

Det. Bailey: OK, we will be continuing our investigation. You are free to go, though we may need to interview you again.

END OF INTERVIEW 


\section{Police Interrogation Transcripts: Vancouver Condition}

Supplemental Vancouver Police Department

Crime Report Form 38.3 Rev. 5/09

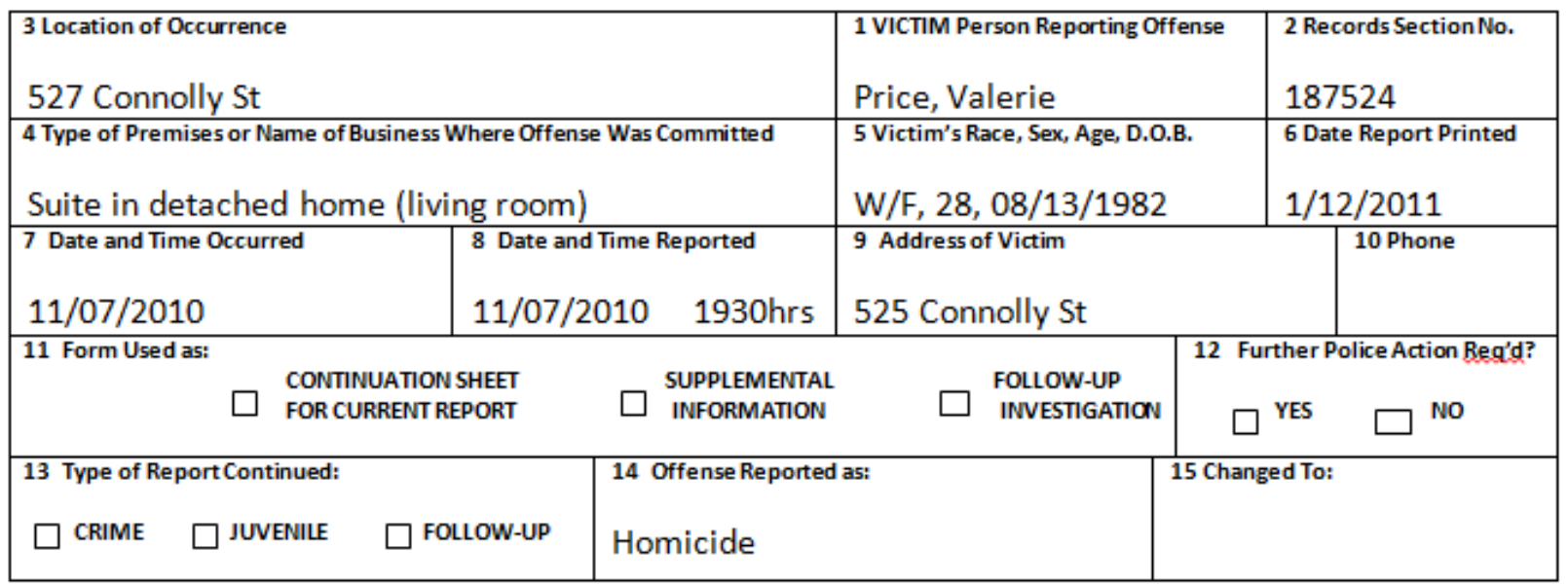

\section{INTERROGATION OF ROBERT HARRIS, 1/12/2011, 11:01PM:}

Det. Bailey: Could you state your full name for the records?

Harris: My name is Robert Harris.

Det. Bailey: Thank you. And where do you live, Mr. Harris?

Harris: 532 Taunton St.

Det. Bailey: And how long have you lived there?

Harris: About 5 years or so.

Det. Bailey: Good, thank you. We are investigating the murder of one of your neighbours,

Valerie Price, on November $7^{\text {th }}, 2010$, approximately two months ago. This is a photo of her

[shows Harris the photo]. Did you know the victim at all, Mr. Harris?

Harris: Yes.

Det. Bailey: How well did you know her? 
Harris: Not very. I mean, I'd recognize her, we'd say hi if we saw each other on the street, but that's about it.

Det. Bailey: How long had you known her?

Harris: I don't know, maybe a couple of years, but I'm really not sure.

Det. Bailey: And how would you characterize your relationship with her?

Harris: I don't know. Acquaintances, I guess.

Det. Bailey: And have you ever been inside her home?

Harris: No.

Det. Bailey: Could you detail for me, to the best of your ability, your whereabouts on November 7, 2010, the day of Ms. Price's murder two months ago. Start at the beginning of your day and continue until the end of that day. Keep in mind that we will be verifying your statement and any lies or omissions that we note will cast further suspicion on you. Murder is a serious crime, Mr. Harris, so really, it's in your best interest to be as honest and forthcoming as possible.

\section{VERSION 1 (Morally Undesirable)}

Harris: I got up between 8:30 and 9, spent the morning at home working on a project for work, watched the football game in the afternoon, then made dinner at home.

Det. Bailey: OK, were you still at home that same evening?

Harris: No.

Det. Bailey: Where were you that evening between 7 and 7:30PM, Mr. Harris?

Harris: I was at the home of my friend Michael Beauregard and his wife Kaitlynn. I think I got there at about twenty to seven, maybe quarter to.

Det. Bailey: Were they home? 
Harris: Michael is away on a business trip, but Kaitlynn was there.

Det. Bailey: OK, we will be checking on that. What were you doing there?

Harris: That night I was in bed with Kaitlynn. We've been having an affair.

Det. Bailey: What time did you return home?

Harris: I think I left around 11:30 or so, and was home by 11:45.

Det. Bailey: And you're sure that is the time you returned home? It could not have been any earlier?

Harris: Yes, I'm positive.

Det. Bailey: Thank you. We're going to need you to provide us with a list of individuals who could support your statement and any physical evidence that could confirm what you say. After that, you are free to go, though we may need to interview you again.

END OF INTERVIEW

\section{VERSION 2 (Morally Neutral)}

Harris: I got up between 8:30 and 9, spent the morning at home working on a project for work, watched the football game in the afternoon, then made dinner at home.

Det. Bailey: OK, were you still at home that same evening?

Harris: No.

Det. Bailey: Where were you that evening between 7 and 7:30PM, Mr. Harris?

Harris: I was at the home of my friend Michael Beauregard and his wife Kaitlynn. I think I got there at about twenty to seven, maybe quarter to.

Det. Bailey: Were they home?

Harris: Michael is away on a business trip, but Kaitlynn was there. 
Det. Bailey: OK, we will be checking on that. What were you doing there?

Harris: That night I was picking up some DVDs I let Michael and Kaitlynn borrow.

Det. Bailey: What time did you return home?

Harris: I think I left around 11:30 or so, and was home by 11:45.

Det. Bailey: And you're sure that is the time you returned home? It could not have been any earlier?

Harris: Yes, I'm positive.

Det. Bailey: Thank you. We're going to need you to provide us with a list of individuals who could support your statement and any physical evidence that could confirm what you say. After that, you are free to go, though we may need to interview you again.

END OF INTERVIEW

\section{VERSION 3 (Morally Desirable)}

Harris: I got up between 8:30 and 9, spent the morning at home working on a project for work, watched the football game in the afternoon, then made dinner at home.

Det. Bailey: OK, were you still at home that same evening?

\section{Harris: No.}

Det. Bailey: Where were you that evening between 7 and 7:30PM, Mr. Harris?

Harris: I was at the home of my friend Michael Beauregard and his wife Kaitlynn. I think I got there at about twenty to seven, maybe quarter to.

Det. Bailey: Were they home?

Harris: Michael is away on a business trip, but Kaitlynn was there.

Det. Bailey: OK, we will be checking on that. What were you doing there? 
Harris: That night I was helping Michael and Kaitlynn move, loading boxes and furniture on the truck.

Det. Bailey: What time did you return home?

Harris: I think I left around 11:30 or so, and was home by 11:45.

Det. Bailey: And you're sure that is the time you returned home? It could not have been any earlier?

Harris: Yes, I'm positive.

Det. Bailey: Thank you. We're going to need you to provide us with a list of individuals who could support your statement and any physical evidence that could confirm what you say. After that, you are free to go, though we may need to interview you again.

END OF INTERVIEW

\section{VERSION 4 (No-Alibi Control)}

Harris: Let me think. [PAUSE] That was about two months ago, so I'm having trouble remembering. What day of the week was that?

Det. Bailey: It was a Sunday.

Harris: In that case, if there was a football game on, I was probably watching that in the afternoon.

Det. Bailey: Where were you that evening between 7 and 7:30PM, Mr. Harris?

Harris: Without checking my calendar, I really can't be sure. I just can't remember where I was that night.

Det. Bailey: Were you at home, or were you out that evening?

Harris: I just don't know. Some Sunday evenings I stay in, sometimes I go out. 
Det. Bailey: So you do not have regular Sunday evening activities?

Harris: No. It varies.

Det Bailey: What kinds of activities might you do on a Sunday night?

Harris: Maybe a drink with friends, or a dinner date.

Det. Bailey: When you do go out on Sundays, what time do you usually return home?

Harris: It depends, usually not after 11:30 or so.

Det. Bailey: OK, we will be continuing our investigation. You are free to go, though we may need to interview you again.

END OF INTERVIEW 
Appendix S: Experiment 2 Questionnaire

Valerie Price Case

NOTE: PARTICIPANTS IN NO-ALIBI CONTROL WILL NOT BE ASKED ABOUT THE ALIBI

1. What is your age? Years

2. In terms of your gender, you would self-identify as (choose all that apply):

$\square$ Female

$\square$ Male

$\square$ Trans*

Queer

Other, please specify

3. What is your ethnicity (choose all that apply)?

- American Indian or Alaska Native

- First Nations Canadian

- Black or African American

- East Asian

- Hispanic or Latino/a

- Native Hawaiian or Other Pacific Islander

- West Asian/Middle Eastern

- White

- Other, please specify

Please answer the following questions as best you can with the limited information from the police file.

4. How honest do you think Robert Harris is being in his description of his whereabouts on the evening in question?

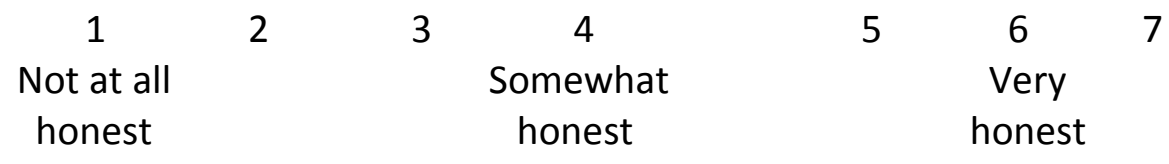

5. How confident are you in your decision for Question \#4?

$\begin{array}{ccccccc}1 & 2 & 3 & 4 & 5 & 6 & 7 \\ \begin{array}{c}\text { Not at all } \\ \text { confident }\end{array} & & \begin{array}{c}\text { Someryhat } \\ \text { confident }\end{array} & & \text { confident }\end{array}$


6. How accurate do you think Robert Harris' statement regarding his whereabouts on the evening in question is? In other words, how well does his statement match his activities on the night in question?

$\begin{array}{ccccccc}1 & 2 & 3 & \begin{array}{c}4 \\ \text { Somewhat } \\ \text { accurate }\end{array} & 5 & 6 & \begin{array}{c}7 \\ \text { Very all }\end{array} \\ \text { accurate } & & & & & \text { accurate }\end{array}$

7. How confident are you in your decision for Question \#6?

$\begin{array}{ccccccc}1 & 2 & 3 & 4 & 5 & 6 & 7 \\ \begin{array}{c}\text { Not at all } \\ \text { confident }\end{array} & & & \begin{array}{c}\text { Somewhat } \\ \text { confident }\end{array} & & & \begin{array}{c}\text { Very } \\ \text { confident }\end{array}\end{array}$

8. When Robert Harris said that he was somewhere else when the crime was committed, his description of what he was doing at the time was probably:

The truth; he was telling the police where he was at the time A lie; he was deliberately giving false information to police An error; he was trying to tell the truth, but was mistaken about his whereabouts

9. Overall, how strong do you think the evidence is against Robert Harris?

$\begin{array}{ccccccc}1 & 2 & 3 & 4 & 5 & 6 & 7 \\ \begin{array}{c}\text { Not at all } \\ \text { strong }\end{array} & & & \begin{array}{c}\text { Somewhat } \\ \text { strong }\end{array} & & & \begin{array}{c}\text { Very } \\ \text { strong }\end{array}\end{array}$

10. Based on the evidence provided to you, how likely do you think it is that Robert Harris committed the crime?

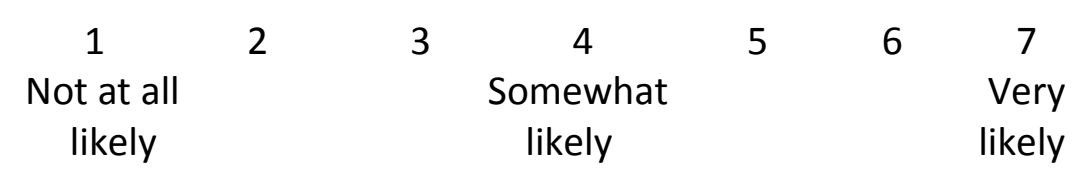

11. If I was asked to provide a verdict in the case, I would vote that Robert Harris is:

_ Guilty Innocent

12. In terms of personality, how similar do you think you are to Robert Harris?

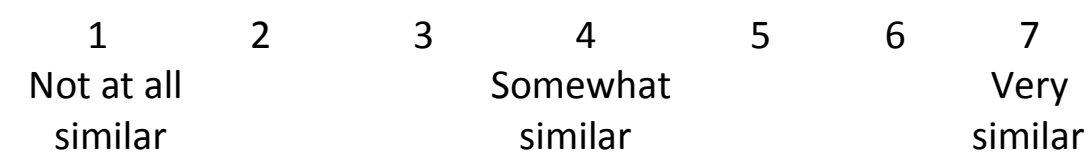


13. How much do you like Robert Harris?

$\begin{array}{ccccccc}1 & 2 & 3 & \begin{array}{c}4 \\ \text { Somewhat }\end{array} & 5 & 6 & \begin{array}{c}7 \\ \text { A lot }\end{array}\end{array}$

14. Overall, I think that Robert Harris is:

1

23

4

5

6

7

8

9

10

11

A very

Neither

A very

bad

good

good

person

nor bad

person

15. What did Robert Harris say he was doing at the time of the crime?

16. Have you ever engaged in the behavior(s) you described in the previous question?<smiles>CC[AsH2]C</smiles>

17. Did Robert Harris provide any evidence to support his statement that he was somewhere else when the crime was committed? If so, please describe.

18. Regarding Robert Harris' activities at the time of the crime, you would evaluate his 
behavior as:

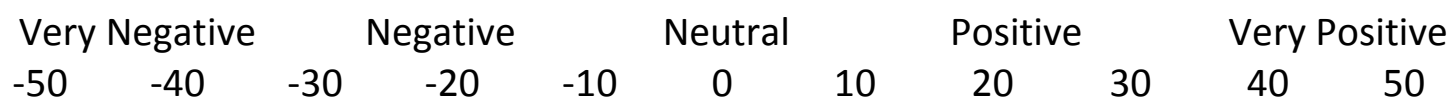

19. Regarding Robert Harris' activities at the time of the crime, if you found out that an adult male in North America did this at least once, your evaluation of that person would be:

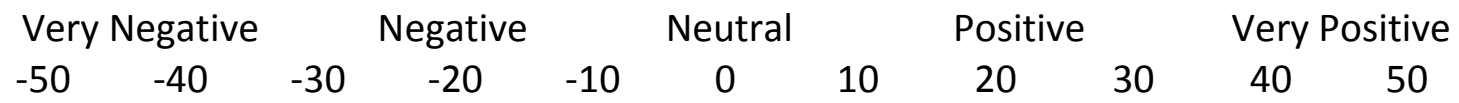

20. Please write below, in your own words, how you define the term ALIBI.

21. Please provide any general comments you have about the case. 
ACT APPEARS HERE

22. Please indicate how you feel about members of the following groups (0-100 VISUAL ANALOG SCALE):

- African Americans

- First Nations/American Indian/Indigenous People

- Caucasian/White People

- Muslims

- Christians

- Jewish People

- Senior Citizens

- Adolescents

- Males

- Females

- Transgender Individuals

- Homosexuals

- Heterosexuals

- Bisexuals 


\section{Appendix T: Experiment 2 Petition Websites}

\section{Seattle Condition}

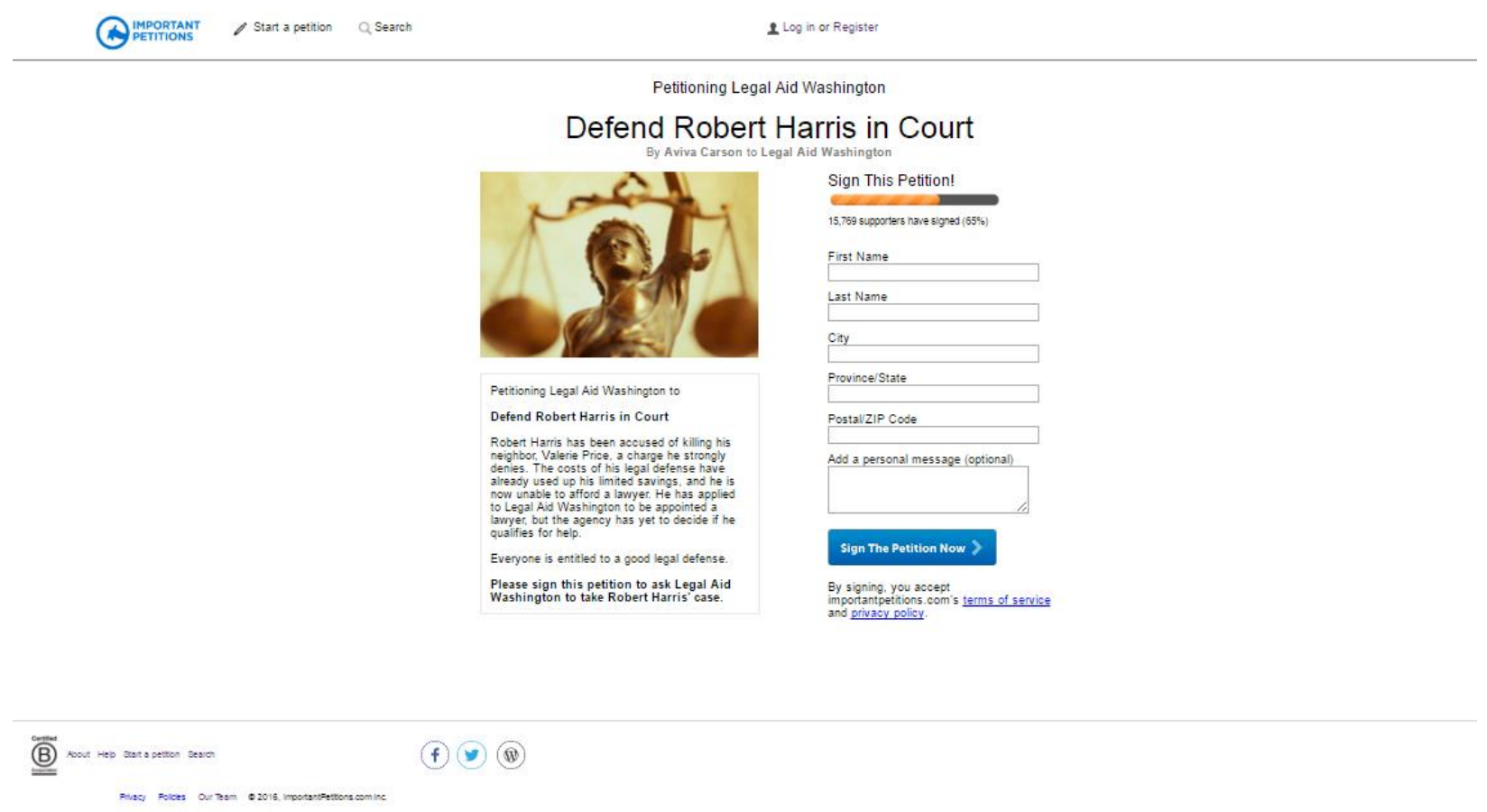

\section{Vancouver Condition}

Petitioning Legal Aid British Columbia Defend Robert Harris in Court

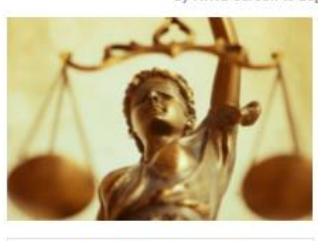

Petritioning Legal Aid Brtitish Columbia to Defend Robert Harris in Court Rober Harris has been accused of kiling his denies. The costs of his legal defense have aready used up his limited savings, and he is to Legal Aid British Columber to be appointed a

Everyone is entitled to a good legal defense. Please sign this petition to ask Legal Aid
British Columbia to take Robert Harris'

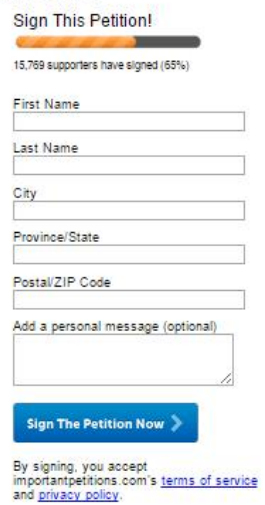

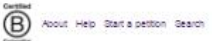

(f) (1) 


\section{Appendix U: Manipulation Check Analyses for Experiment 2}

As in Experiment 1, participants in the conditions that included an alibi were asked to rate the behaviour that the suspect described in his alibi on a Likert scale from 1 (Very Negative) to 11 (Very Positive). A between-subjects univariate ANOVA showed that Alibi Moral Desirability affected participants' judgments about the alibi behaviour, $F(2,215)=98.95, p<$ $.001, \eta_{\mathrm{p}}^{2}=.48$. All follow-up comparisons were statistically significant using a Holm-Bonferroni correction. The Morally Undesirable alibi $(M=3.27, S D=1.70)$ was rated significantly more negatively than the Neutral alibi $(6.24, S D=1.80), t(144)=-10.25, p<.001, d=-1.70$, and the Morally Desirable alibi $(M=6.85, S D=1.44), t(145)=-13.74, p<.001, d=-2.27$. The Neutral alibi was also rated more negatively than the Morally Desirable alibi, $t(141)=2.23, p=.027, d=$ 0.37, though the effect size is substantially smaller than the effect sizes for the other two comparisons.

It is also important to establish that the Morally Desirable, Morally Undesirable, and Neutral alibis are perceived as such. To accomplish this, participants' mean ratings of the alibi behaviour were compared to the midpoint of the scale (i.e., 6) for each alibi narrative. The mean rating for the behaviour of the Morally Undesirable alibi was significantly below the midpoint, $t(74)=-13.90, p<.001, d=-1.60$. The mean rating for the Morally Desirable alibi was significantly higher than the midpoint, $t(71)=4.99, p<.001, d=0.59$. The mean rating for the Neutral alibi was not significantly different from the midpoint, $t(70)=1.12, p=.266, d=0.13$.

Participants were also asked to rate their feelings about an adult male who had engaged in the activities that the suspect described in his alibi on a scale from 1 (Very Negative) to 11 (Very Positive). Alibi Moral Desirability significantly affected this judgment as well, $F(2,214)=$ 45.09, $p<.001, \eta_{\mathrm{p}}^{2}=.30$. As above, the Morally Undesirable alibi $(M=3.52, S D=1.93)$ was 
rated significantly more negatively than the Neutral alibi $(M=6.18, S D=1.76), t(143.78)=-$ 8.71, $p<.001, d=-1.42$, and the Morally Desirable alibi $(M=6.28, S D=2.28), t(144)=-9.91, p$ $<.001, d=-1.31$. However, there was no significant difference between the Morally Desirable and Neutral alibis, $t(131.50)=.29, p=.773, d=0.05$.

As with participants' ratings of the alibi behaviour, their ratings of an individual engaged in that behaviour were compared to the midpoint of the scale to establish whether participants' views of the moral desirability of the various activities was consistent with what would be anticipated. An individual engaged in the Morally Undesirable activities was rated significantly below the midpoint of the scale, $t(74)=-11.12, p<.001, d=-1.28$. The mean ratings for the Morally Desirable, $t(71)=1.04, p=.302, d=0.12$, and Neutral activities, $t(70)=.88, p=.383, d$ $=0.10$, were not significantly different from the midpoint.

In general, it seems that the Morally Undesirable and Neutral alibis are being perceived as intended by the manipulation, but the Morally Desirable alibi is not. Descriptive statistics for this condition suggest that at least some participants viewed the Morally Desirable alibi negatively and many viewed it as neutral. Participants' ratings of the behaviour ranged from 4 to $11 ; 9.7 \%$ of participants rated the behaviour negatively (i.e., either 4 or 5), $47.2 \%$ of participants rated the behaviour as neutral (i.e., 6), and $43.1 \%$ of participant rated the behaviour as positive (i.e., 7-11). Judgments about an individual engaged in the behaviour ranged from 1 to $11 ; 22.5 \%$ of participants rated the actor negatively (i.e., 1-5), 42.3\% of participants rated the actor neutrally (i.e., 6), and $35.2 \%$ of participants rated the actor positively (i.e., 7-11).

Finally, within each suspect race condition, there were two photographs that participants could be shown. It is important to establish whether the photos were perceived similarly within the two suspect race conditions. For the two photos of White suspects, participants did not differ 
in their ratings of how much they liked the suspect, $t(146)=-1.24, p=.216, d=-0.21$, nor whether they thought the suspect was a good or bad person, $t(146)=.57, p=.567, d=0.09$. For the two photos of the First Nations suspect, participants did not differ in how much they liked the suspect, $t(130)=.87, p=.385, d=0.15$, but there was a trend for participants' ratings the suspect in the first photo $(M=5.49, S D=1.44)$ to be more negative than their ratings of the suspect in the second photo $(M=5.93, S D=1.12)$ on an 11-point Likert scale, $t(130.98)=-1.97, p=.051$, $d=-0.34$. 


\section{Appendix V: Analyses for Participant Race/Ethnicity in Experiment 2}

As in Experiment 1, the sample for Experiment 2 was racially/ethnically diverse, with approximately $55 \%$ of participants identifying as not being White. Unfortunately, too few participants reported any particular non-White identity to generate sufficient statistical power to allow the groups to be analyzed separately. Consequently, all analyses in this section will compare participants who identified solely as White to participants who identified as any other group or combination of groups. Overall, $39.5 \%$ of participants identified as only being White. The remaining $60.4 \%$ of participants were in the People of Colour (POC) group, and the one participant who chose not to respond to this item was excluded from analyses. Thus, all analyses tested a 2 (Participant Race/Ethnicity: White, POC) X 2 (Suspect Race: White, First Nations) design.

Statistical results for the continuous dependent measures are available in Table V1. The only result of note is a marginally significant trend for POC $(M=4.76, S D=1.39)$ to be more confident in their judgments of suspect honesty than White participants $(M=4.41, S D=1.68)$.

Alibi classification. A multinomial logistic regression was used to determine whether participants' classification of the alibi as True, Fabricated, or Mistaken was associated with Suspect Race or Participant Race/Ethnicity. As in previous analyses, Fabricated was the reference category for the outcome variable. The nonsignificant Pearson, $\chi^{2}(2)=2.33, p=.311$, and deviance, $\chi^{2}(2)=2.35, p=.309$, suggest that the model is a poor fit for the data. This is consistent with the results for the specific predictors, none of which were statistically significant. For the logit comparing True and Fabricated classifications, no significant effects were found for Suspect Race, $b=-54, S E=.31$, Wald $=3.05, p=.081$, Odds Ratio $=0.58$, or for Participant Race/Ethnicity, $b=-.29, S E=.31$, Wald $=.86, p=.354$, Odds Ratio: 0.75 . For the logit 
comparing Mistaken and Fabricated alibi classifications, no significant effects were found for Suspect Race, $b=-.52, S E=.39$, Wald $=1.78, p=.182$, Odds Ratio $=0.60$, or Participant Race/Ethnicity, $b=-.73, S E=.41$, Wald $=3.27, p=.070$, Odds Ratio $=0.48$. Although this latter effect approached significance, and would suggest that White participants were somewhat more likely than People of Colour to indicate that the alibi was Mistaken rather than Fabricated, the overall model fit was poor, so the result of this particular predictor may not be reliable.

Table V1

ANOVA results comparing responses of POC and White participants on continuous outcomes

\begin{tabular}{|c|c|c|c|c|c|}
\hline Dependent Measure & Effect & $d f$ & $F$ & $p$ & $\eta_{\mathrm{p}}^{2}$ \\
\hline \multicolumn{6}{|l|}{ Suspect honesty } \\
\hline & Participant Race & 1,277 & 1.52 & .218 & .01 \\
\hline & Suspect Race & 1,277 & .73 & .394 & $<.01$ \\
\hline & Participant Race X Suspect Race & 1,277 & .58 & .446 & $<.01$ \\
\hline \multicolumn{6}{|c|}{$\begin{array}{l}\text { Confidence in suspect } \\
\text { honesty }\end{array}$} \\
\hline & Participant Race & 1,277 & 3.42 & .066 & .012 \\
\hline & Suspect Race & 1,277 & .95 & .330 & $<.01$ \\
\hline & Participant Race X Suspect Race & 1,277 & .03 & .860 & $<.01$ \\
\hline \multicolumn{6}{|l|}{ Alibi accuracy } \\
\hline & Participant Race & 1,216 & .34 & .560 & $<.01$ \\
\hline & Suspect Race & 1,216 & .25 & .615 & $<.01$ \\
\hline & Participant Race X Suspect Race & 1,216 & 2.61 & .108 & .01 \\
\hline \multicolumn{6}{|l|}{$\begin{array}{l}\text { Confidence in alibi } \\
\text { accuracy }\end{array}$} \\
\hline & Participant Race & 1,215 & .70 & .404 & $<.01$ \\
\hline & Suspect Race & 1,215 & 1.30 & .255 & .01 \\
\hline & Participant Race X Suspect Race & 1,215 & $<.01$ & .992 & $<.01$ \\
\hline \multicolumn{6}{|c|}{$\begin{array}{l}\text { Strength of evidence } \\
\text { against the suspect }\end{array}$} \\
\hline & Participant Race & 1,278 & 2.35 & .127 & .01 \\
\hline & Suspect Race & 1,278 & .45 & .503 & $<.01$ \\
\hline & Participant Race X Suspect Race & 1,278 & .64 & .423 & $<.01$ \\
\hline \multicolumn{6}{|l|}{ Likelihood of guilt } \\
\hline & Participant Race & 1,277 & .49 & .483 & $<.01$ \\
\hline & Suspect Race & 1,277 & .58 & .447 & $<.01$ \\
\hline & Participant Race X Suspect Race & 1,277 & 2.67 & .103 & .01 \\
\hline
\end{tabular}


Verdict choice. A loglinear analysis with backwards elimination was computed with Suspect Race, Participant Race/Ethnicity, and Verdict Choice. The chi-square test on the likelihood ratio was not significant, $\chi^{2}(5)=5.20, p=.392$, suggesting that the model is a good fit for the data. All expected cell counts were within acceptable limits. Once unnecessary terms were removed, the model comprised only main effects of Participant Race/Ethnicity and Verdict Choice. These results indicate that there were significantly more guilty verdicts $(36.3 \%)$ than innocent verdicts (63.7\%), and a significantly greater number of participants were in the POC group $(60.4 \%)$ than in the White group $(39.5 \%)$. No interaction terms that included Verdict Choice remained in the model, suggesting that there is no significant association between it and any of the other variables.

Petition signing. A loglinear analysis with backwards elimination was computed with Suspect Race, Participant Race/Ethnicity, and Petition Signing. The chi-square test on the likelihood ratio showed that the model was a good fit for the data, $\chi^{2}(2)=1.89, p=.388$. Interactions between Suspect Race and Petition Signing and between Participant Race/Ethnicity and Petition Signing remained in the model after unnecessary terms were removed. As noted earlier, participants were significantly more likely to sign the petition when the suspect was First Nations $(61.1 \%)$ than when the suspect was White $(47.6 \%), \chi^{2}(1)=4.25, p=.048, \varphi=-.14$. As well, the interaction shows that White participants (64.2\%) were significantly more likely to sign the petition than were POC $(47.1 \%)$. The overall rate of petition signing across the sample was $53.9 \%$. 


\section{Appendix W: Analyses of Participants' General Feelings about White and First Nations People in Experiment 2}

In general, participants' feelings about First Nations and White people were fairly positive and the distributions were highly skewed to the left. For perceptions of First Nations people, the mean rating was $83.39(S D=19.14 ; M d n=90$; Range: $28-100)$. The most common response was 100 , with $38.7 \%$ of the sample providing this rating. Nearly half of the sample (49.0\%) provided ratings of 90 or higher. For perceptions of White people, the mean rating was $82.14(S D=20.32, M d n=90 ;$ Range: 27-100). Again, the most common response was 100, with $36.9 \%$ of the sample providing this rating, and nearly half of the sample (48.1\%) provided ratings that were 90 or higher. The two ratings were significantly positively correlated, $r=.568$, $p<.001$, but are not identical. Examination of a scatterplot (see Figure W1) shows the positive linear relationship and that there are a number of participants who provided high ratings for one group and moderate or low ratings for the other group. This suggests that the scores do represent their feelings about the particular groups rather than their feelings about people as a whole, irrespective of their membership in particular identifiable groups.

Regression models were computed to investigate whether participants' perceptions of First Nations and of White people generally influenced their judgments in the case. It was hypothesized that participants' feelings about First Nations people in general would predict their judgments about the First Nations suspect and that their feelings about White people in general would predict their judgments about the White suspect. To test these hypotheses, regressions were computed separately for participants who saw the First Nations suspect and those who saw the White suspect. Each regression was computed twice: in one model, only the rating for the 


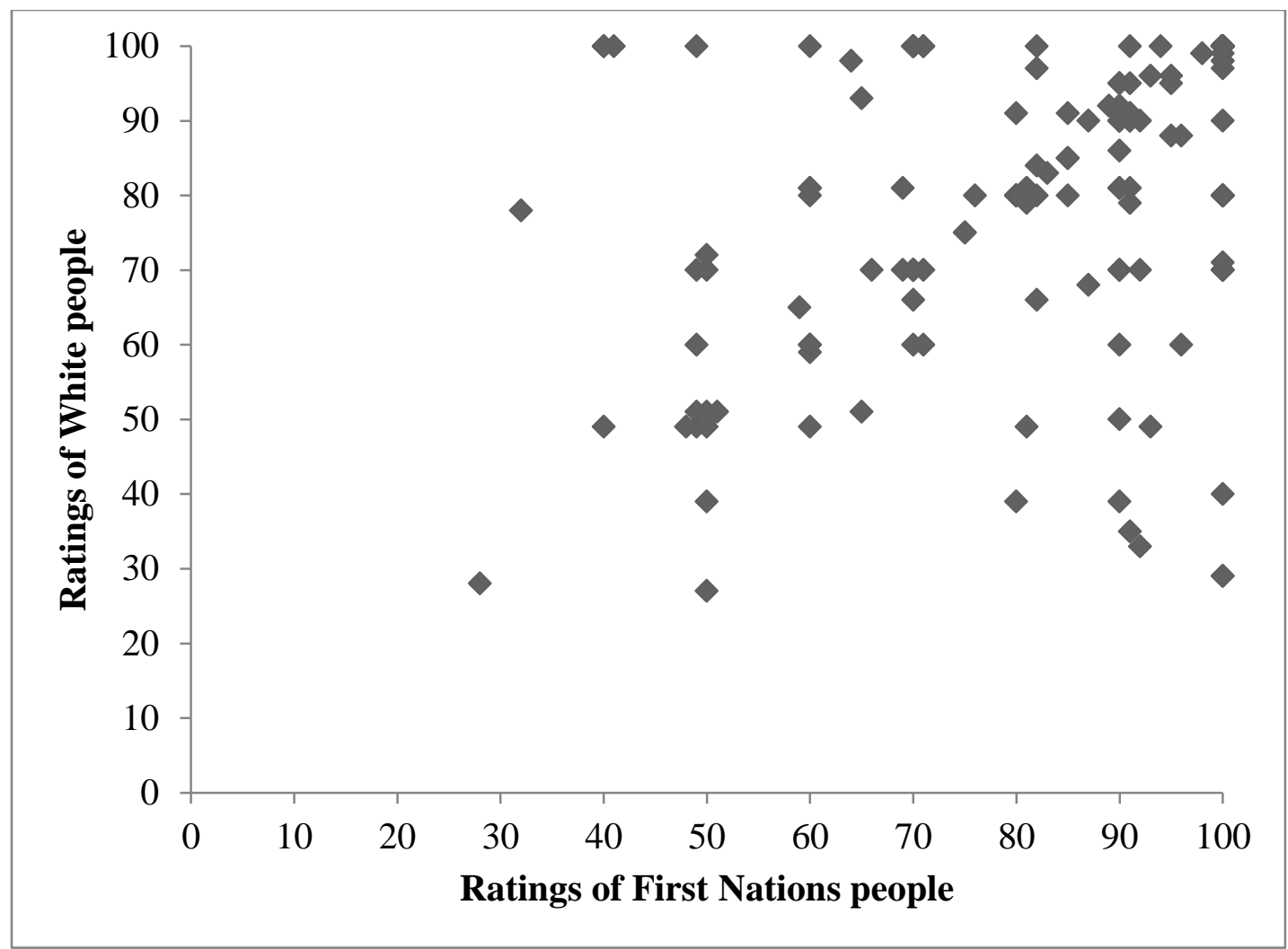

Figure W1

Correlation between participants' general feelings about First Nations and White people

suspect's group was included, and in the other model, both ratings were included. The latter models were to determine whether any effects found in the first model persisted when the other ratings were controlled. Statistical results are presented in Tables W1 and W2, and a brief summary is provided below. Overall, there is not compelling, consistent evidence to suggest that participants' ratings on the thermometer items are related to their legal judgments about First Nations or White suspects. Only two significant regression models were found. Both were for the White suspect condition. First, more positive feelings about First Nations people were associated with greater confidence in suspect honesty judgments in the multiple regression model. This effect was not found in the simple regression model. Second, more positive feelings about White 
people resulted in a greater likelihood that the participant would sign the petition in support of the suspect. It is worth noting again that the majority of participants rated both First Nations and White people very highly, and some of the models violated the assumptions of homoscedasticity and normality of residuals. It is possible that participants' general feelings about the suspect's racial/ethnic group would be a significant predictor of legal judgments if there was greater variability in the predictors included in the models. In other words, the lack of covariation between predictors and the outcomes may be due to insufficient variation in the predictor variables rather than a genuine absence of effect. However, the general conclusion from the current data is that participants' reported feelings about First Nations and White people do not affect their judgments about First Nations or White suspects. 
Table W1

Simple and multiple linear regression models using participants' general feelings about White and/or First Nations people to predict their judgments about White or First Nations suspects.

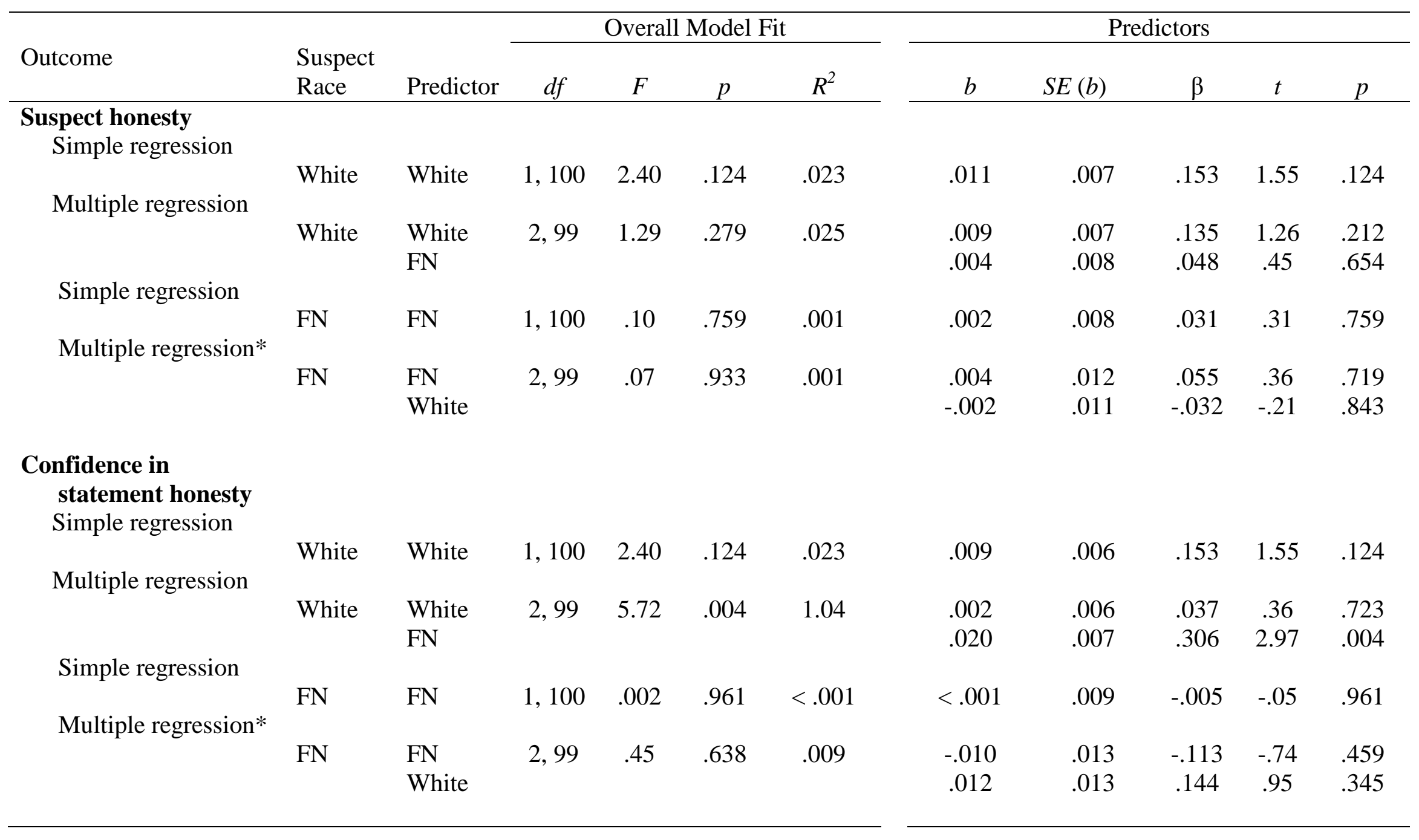




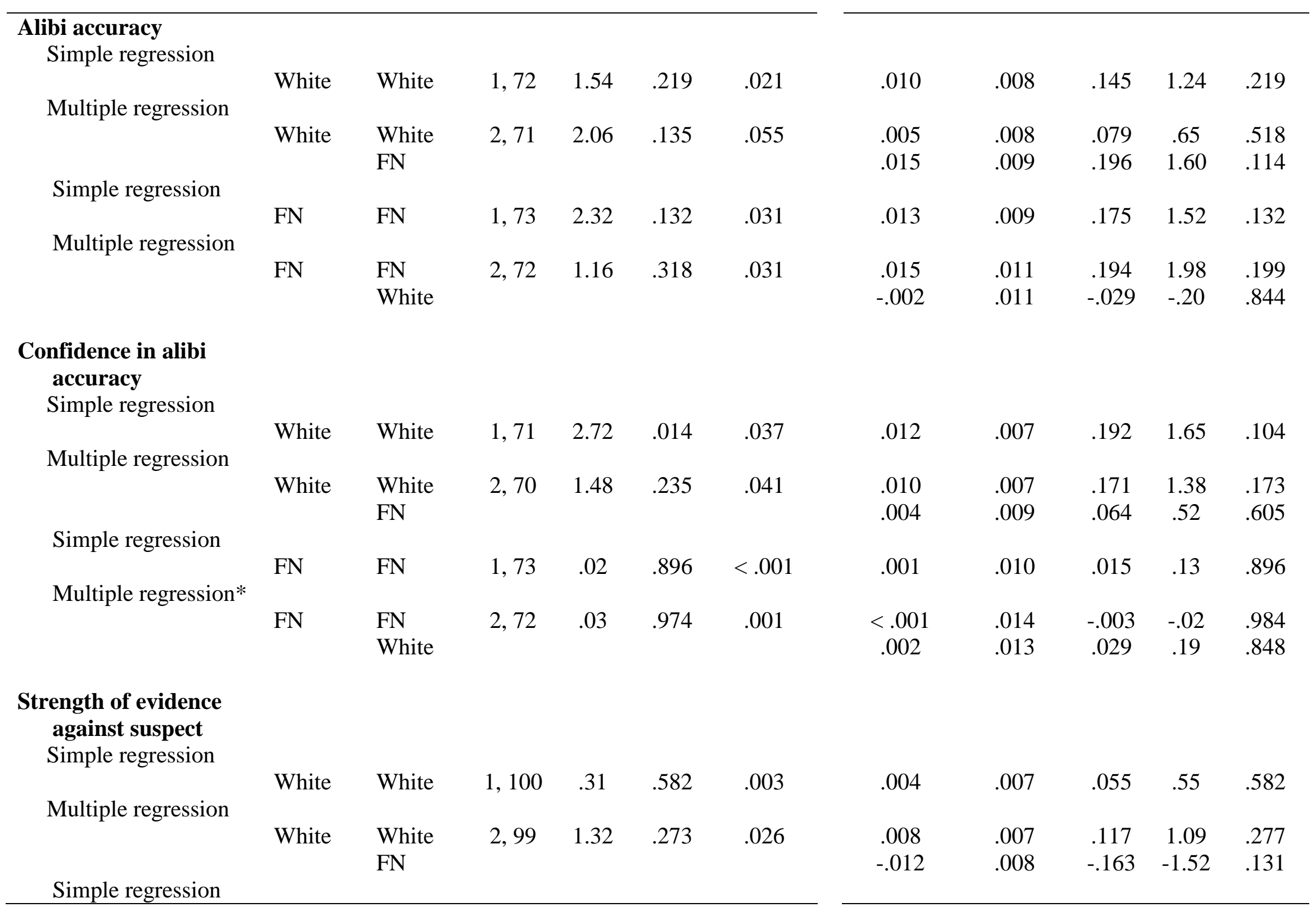




\begin{tabular}{|c|c|c|c|c|c|c|c|c|c|c|c|}
\hline \multirow{2}{*}{ Multiple regression } & $\mathrm{FN}$ & FN & 1,100 & .21 & .647 & .002 & .004 & .008 & .046 & .46 & .647 \\
\hline & FN & $\begin{array}{l}\text { FN } \\
\text { White }\end{array}$ & 2,99 & .90 & .410 & .018 & $\begin{array}{c}.016 \\
-.016\end{array}$ & $\begin{array}{l}.013 \\
.012\end{array}$ & $\begin{array}{c}.188 \\
-.190\end{array}$ & $\begin{array}{c}1.25 \\
-1.26\end{array}$ & $\begin{array}{l}.214 \\
.211\end{array}$ \\
\hline \multicolumn{12}{|l|}{$\begin{array}{l}\text { Likelihood of suspect } \\
\text { guilt } \\
\text { Simple regression }\end{array}$} \\
\hline \multirow{2}{*}{ Multiple regression } & White & White & 1,100 & .68 & .413 & .007 & -.006 & .007 & -.082 & .82 & .413 \\
\hline & White & $\begin{array}{l}\text { White } \\
\text { FN }\end{array}$ & 2,99 & 1.93 & .151 & .037 & $\begin{array}{l}-.001 \\
-.014\end{array}$ & $\begin{array}{l}.007 \\
.008\end{array}$ & $\begin{array}{l}-.010 \\
-.190\end{array}$ & $\begin{array}{c}-.09 \\
-1.78\end{array}$ & $\begin{array}{l}.928 \\
.078\end{array}$ \\
\hline Simple regression & FN & $\mathrm{FN}$ & 1,99 & .27 & .607 & .003 & -.004 & .008 & -.052 & -.52 & .607 \\
\hline Multiple regression & $\mathrm{FN}$ & $\begin{array}{l}\text { FN } \\
\text { White }\end{array}$ & 2,98 & .29 & .750 & .006 & $\begin{array}{l}.001 \\
-.006\end{array}$ & $\begin{array}{l}.012 \\
.011\end{array}$ & $\begin{array}{c}.012 \\
-.085\end{array}$ & $\begin{array}{c}.08 \\
-.56\end{array}$ & $\begin{array}{l}.937 \\
.577\end{array}$ \\
\hline
\end{tabular}

Note. Diagnostic statistics showed that the multiple regression model computed for White suspects predicting suspect honesty was mildly heteroscedastic; there appears to be greater variance in the residuals at higher regression predicted values than at some of the lower predicted values. Additionally, models denoted with an * showed evidence of non-normal residuals or non-normal residuals and heteroscedasticity. Transformations were unsuccessful at normalizing the data, so results of these model should not be generalized beyond the current data. 
Table W2

Simple and multiple binary logistic regression models using participants' general feelings about White and/or First Nations people to predict their judgments about White or First Nations suspects

\begin{tabular}{|c|c|c|c|c|c|c|c|c|c|c|c|}
\hline \multirow[b]{2}{*}{ Outcome } & \multirow[b]{2}{*}{$\begin{array}{l}\text { Suspect } \\
\text { Race }\end{array}$} & \multirow[b]{2}{*}{ Predictor } & \multicolumn{4}{|c|}{ Overall Model Fit } & \multicolumn{5}{|c|}{ Predictors } \\
\hline & & & $d f$ & $\chi^{2}$ & $p$ & $R^{2}$ & $b$ & $S E(b)$ & Wald & $p$ & $\begin{array}{l}\text { Odds } \\
\text { Ratio }\end{array}$ \\
\hline \multicolumn{12}{|l|}{$\begin{array}{l}\text { Verdict choice } \\
\text { Simple reoression }\end{array}$} \\
\hline \multirow{2}{*}{ Multiple regression } & White & White & 1 & .075 & .784 & .001 & -.003 & .010 & .075 & .784 & .997 \\
\hline & White & $\begin{array}{l}\text { White } \\
\text { FN }\end{array}$ & 2 & 2.16 & .339 & .029 & $\begin{array}{l}-.009 \\
.017\end{array}$ & $\begin{array}{l}.011 \\
.012\end{array}$ & $\begin{array}{l}.642 \\
2.05\end{array}$ & $\begin{array}{l}.423 \\
.152\end{array}$ & $\begin{array}{c}.991 \\
1.017\end{array}$ \\
\hline & $\mathrm{FN}$ & $\mathrm{FN}$ & 1 & 002 & 967 & $<001$ & $<001$ & 011 & 002 & 967 & 100 \\
\hline 1 & $\mathrm{FN}$ & $\begin{array}{l}\text { FN } \\
\text { White }\end{array}$ & 2 & .07 & .965 & .001 & $\begin{array}{l}-.004 \\
.004\end{array}$ & $\begin{array}{l}.017 \\
.019\end{array}$ & $\begin{array}{l}.050 \\
.069\end{array}$ & $\begin{array}{l}.823 \\
.792\end{array}$ & $\begin{array}{l}.996 \\
1.004\end{array}$ \\
\hline
\end{tabular}

\section{Petition signing}

Simple regression

Multiple regression

\begin{tabular}{lllllllllll} 
White & White & 1 & 4.42 & .036 & .069 & -.023 & .011 & 4.16 & .041 & .977 \\
\multirow{2}{*}{ White } & White & 2 & 4.45 & .108 & .007 & -.024 & .012 & 3.87 & .049 & .977 \\
& FN & & & & & .002 & .013 & .032 & .859 & 1.002
\end{tabular}

Simple regression

Multiple regression

$\begin{array}{lllllllllll}\text { FN } & \text { FN } & 1 & .28 & .597 & .005 & .006 & .012 & .273 & .601 & 1.006 \\ \text { FN } & \text { FN } & 2 & 3.01 & .222 & .048 & .029 & .020 & 2.16 & .142 & 1.029 \\ & \text { White } & & & & & -.029 & .019 & 2.384 & .123 & .971\end{array}$

Note. The Nagelkerke $R^{2}$ is reported. Also, note that verdict choice is coded as $1=$ Guilty, 2 = Innocent. Petition Signing is coded as 1 $=$ Signed, 2 = Not Signed. 


\section{Appendix X: Psychometric Analyses of the ACT for Experiment 2}

The psychometric properties of the ACT were investigated prior to the computation of any inferential tests. In total, 272 participants completed the entire ACT. Participants who did not respond to all relevant items were excluded from the analyses. The overall scale achieved very good internal consistency $(\alpha=.851)$. The alpha value was almost identical to the alpha observed in Experiment $1(\alpha=.853)$. Examination of the specific items showed that the alpha level would increase slightly if one item ("It's great that many young people today are prepared to defy authority" - reverse scored) was removed. The alpha would decrease if any of the other items were removed. This item and one other ("strong, tough government will harm not help our country" - reverse scored) were the only items that showed item-total correlations below 3 . However, the item-total correlations may be low because they represent how well the item correlates with other items in the same subscale as well as with items on the other subscales. Consequently, these two items were flagged as potentially problematic.

The alpha scores for each of the subscales was lower than the overall alpha, but this is expected given the reduction of the number of items included in the analysis. The Conservatism subscale showed acceptable internal consistency $(\alpha=.732, N=280)$. The alpha was slightly lower than the alpha observed in Experiment $1(\alpha=.747 ; N=294)$. The item identified earlier regarding the willingness of young people to defy authority showed a poor inter-item correlation $(r=.270)$, only correlated well with one other item on the subscale $(r=.471$, all other $r<.256)$, and the alpha would increase to .750 if this item was deleted. This is the same item that was removed from the scale in Experiment 1. Consequently, the item was removed.

The Traditionalism subscale showed very good internal consistency $(\alpha=.820, N=280)$.

The scale had greater internal consistency in Experiment 2 than it did in Experiment $1(\alpha=.797$, 
$N=287$ ). One item ("nobody should stick to the 'straight and narrow.' Instead people should break loose and try out lots of different ideas and experiences" - reverse scored) showed a poor inter-item correlation $(r=.197)$ and correlated poorly with all other items on the subscale (all $r<$ .202). As well, the alpha would increase to .854 if the item was deleted. This item was also deleted from the scale in Experiment 1. Consequently, the item was removed from the scale in the current experiment.

The Authoritarianism subscale showed somewhat poor internal consistency $(\alpha=.691, N$ $=275)$, though it is higher than the results from Experiment $1(\alpha=.679, N=.297)$. The alpha would increase to .711 if one item ("strong, tough government will harm not help our country" reverse scored) was deleted. This item showed a poor item-total correlation $(r=.221)$ and correlated poorly with the other items on the subscale (all $r<.288$ ). This item was removed from the scale in Experiment 1. This item was also removed from the scale in the current experiment.

When the total scale was examined with those three items removed, the scale achieved internal consistency that was slightly higher than the alpha observed prior to the items' removal $(\alpha=.856, N=274)$. The item-total correlations were all within the acceptable range (all $r>$ .305). For one item, the alpha would remain unchanged, and for another item, the alpha would only decrease by .01 if the item was removed. The correlations between these items and other items on the same subscale are lower than ideal, and they do not correlate well with items on the other subscales. However, both are from the Conservatism subscale and removing them would result in a subscale with only three items. As a result, they were retained and total scores for the overall scale and for the subscales were computed for a 15-item ACT, rather than on the original 18-item version. As in Experiment 1, participants who did not respond to specific items were not given scores where the item was used to calculate a total score. 
Total possible scores on the ACT ranged from 15 to 105 . The average score $(M=53.12$, $S D=14.21, N=274)$ was slightly below the midpoint of 60 , and observed scores ranged from 17 to 82 . This average was nearly identical to the average from Experiment $1(M=55.74, S D=$ 13.78 , Range $=23-98, N=288)$. For Conservatism, the mean score was $17.70(S D=5.59$, Range $=5-34, N=274)$; for Traditionalism, the mean score was $16.39(S D=7.45$, Range $=5-35, N=$ 274); for Authoritarianism, the mean score was $19.01(S D=4.98$, Range $=5-35, N=274)$. Scores on the subscales represent nearly the entire range of possible scores (5 to 35 ). Scores on the total ACT, the Conservatism subscale, and the Authoritarianism subscale were normal. Scores on the Traditionalism subscale were slightly skewed to the right, but not to such an extent that it is likely to cause problems with inferential tests. Observed mean scores in the present study were similar to those observed in Experiment 1 for the Conservatism subscale $(M=18.19$, $S D=5.47$, range: $5-32, N=296)$ and the Traditionalism subscale $(M=17.10, S D=6.90$, range: $5-34, N=290)$. Scores in the current study are slightly lower than those observed in Experiment 1 for the Authoritarianism subscale $(M=20.42, S D=4.76$, range: $5-35, N=297)$. 


\section{Appendix Y: Inferential Tests Based on Qualitative Responses for Experiment 2}

It is possible that participants' different understandings of what an alibi is affected their judgments about the legal aspects of the case. Differences in responses between participants who understood that an alibi referred to a narrative regarding where a suspect was during the time of a crime and participants whose responses emphasized evidence to support a narrative were investigated (see Table Y1 for statistical results of continuous outcome variables). None of the independent-samples $t$-tests showed significant differences between groups. As well, Verdict Choice, $\chi^{2}(1)=.26, p=.633, \varphi=-.039$, and participants' petition signing behaviour, $\chi^{2}(1)=$ $1.00, p=.328, \varphi=.082$, were not significantly related to their understanding of the term "alibi." Table Y1

Comparison of legal judgments between participants who provided correct definitions and participants who emphasized an alibi as evidence.

\begin{tabular}{lcccccc}
\hline Outcome & $d f$ & $t$ & $p$ & $d$ & $M 1(S D)$ & $M 2(S D)$ \\
\hline Statement honesty & 164 & .18 & .861 & 0.02 & $4.27(1.42)$ & $4.24(1.24)$ \\
Confidence in statement honesty & 164 & .92 & .360 & 0.14 & $4.80(1.46)$ & $4.60(1.38)$ \\
Accuracy & 132 & -.83 & .407 & -0.14 & $3.97(1.39)$ & $4.16(1.26)$ \\
Confidence in accuracy & 132 & .44 & .663 & 0.08 & $4.82(1.35)$ & $4.71(1.44)$ \\
Strength of evidence against the & 165 & .54 & .593 & 0.08 & $3.44(1.73)$ & $3.31(1.42)$ \\
$\quad$ suspect & & & & & & \\
Likelihood of guilt & 165 & .02 & .986 & 0.01 & $3.67(1.43)$ & $3.66(1.44)$ \\
\hline
\end{tabular}

Note. M1 refers to participants who referred to the alibi as a narrative regarding a suspect's whereabouts at the time of a crime, whereas $\mathrm{M} 2$ refers to the participants whose responses emphasized the evidence supporting a statement. 


\section{Appendix Z: Experiment 2 Debrief Form}

\section{Debriefing - Criminal Investigation 2}

The social psychology study that you just participated in examines the factors associated with the evaluation of an alibi. An alibi is the explanation a suspect gives to the police regarding his or her whereabouts when a crime is committed. The Innocence Project (http://www.innocenceproject.org/), an organization that helps the wrongly convicted, found that in approximately $25 \%$ of their DNA exoneration cases, the defendant presented an alibi that wasn't believed. Previous research has demonstrated that alibi evidence (that is ultimately true) is often considered 'weak' by the justice system and therefore ignored (Burke, Turtle and Olson, 2007). As well, The Innocence Project shows that members of visible minority or marginalized groups are over-represented within the justice system. In Canada, First Nations people make up approximately $4 \%$ of the adult population, but represent $18-20 \%$ of incarcerated adults.

A lot of studies have focused on the strength of corroborating evidence to explain why some alibis are believed, while others are not. For example, an alibi that is confirmed by a stranger's testimony and physical evidence that would be hard to fabricate will be believed more often than an alibi corroborated by a relative of the suspect with no physical evidence to back it up. In this study, we were interested in how the content of the alibi and the suspect's racial/ethnic group (the independent variables) influenced whether the alibi was believed and what people thought was the probability that the suspect was guilty. To accomplish this, we created six different versions of the case. All participants read the same basic information, but the alibi the suspect offered was either: (a) morally undesirable, (b) morally desirable, or (c) morally neutral. Additionally, the suspect was either (a) White Canadian or (b) First Nations Canadian.

Our first hypothesis was that morally undesirable alibis would be rated as more believable and the suspect would be judged to be less likely to be guilty than with neutral alibis. We expected that participants would interpret these alibis as evidence of honesty - why would a suspect admit to something that makes him look bad unless it was the truth? We also expected that participants would be more likely to agree to sign the petition supporting the suspect if the alibi was morally undesirable, because they thought the alibi was true and the suspect was innocent.

Our second hypothesis was that alibis provided by First Nations Canadians would be rated as less believable and the suspect would be seen as more likely to be guilty than when the alibi was provided by a White Canadian. We also expected that participants would be more likely to sign the petition if the suspect was a White Canadian.

Our third hypothesis was that the differences in believability between morally desirable, morally undesirable, and morally neutral alibis might be different depending on whether the suspect was a White or First Nations Canadian.

The results will allow us to see if the content of suspects' alibis and the racial/ethnic identity of the alibi provider have an influence on juror decisions of guilt and alibi believability and their petition-signing behaviour (the dependent variables). This information may allow us to help the justice system evaluate and use alibi information more appropriately. 
If you know of others who will be participating in this experiment, please refrain from discussing it with them. We do not want our future participants to be aware of the procedures and expected findings.

Thank you for participating in our research. If you have any questions about this study, please contact:

Sara Cowan, MA

sara.cowan@psych.ryerson.ca

416-979-5000 x2190
Tara Burke, Ph.D.

tburke@psych.ryerson.ca

416-979-5000 x6519

If you any have questions about receiving your Psychology 102/202 credit for participation please contact: thepool@ psych.ryerson.ca 


\section{Appendix AA: Experiment 3 Recruitment Information}

\section{Mechanical Turk Recruitment Information}

Title: Responses to Media Reports about Criminal Trials

Description: Read a brief newspaper article about an upcoming trial, then provide information about your impressions of the case and fill out personality questionnaires.

Keywords: psychology, crime, criminal trials, impressions

Time allotted: 40 minutes

Reward: \$1

Qualifications: Must be 18+ years, location must be US or Canada.

Individuals who choose to potentially participate/seek more information will read the following:

The purpose of this study is to examine how people form impressions about criminal cases and defendants from media accounts. You are being invited to participate in this study because you are enrolled in Amazon's Mechanical Turk, are at least 18 years of age, and reside in the United States or Canada. You should not participate if you are not at least 18 years old, or if you currently live outside of the United States or Canada. Your participation will last for up to 40 minutes. If you agree to participate, you will be shown a newspaper article describing an upcoming criminal trial. You will be asked questions about your impressions of the case, and will be asked to complete two personality questionnaires.

The study is hosted on the Qualtrics website, and you will see a full informed consent document there.

If you have any questions about the study now, or at any time, please contact:

Sara Cowan, MA (sara.cowan@psych.ryerson.ca)

Tara M. Burke, PhD (tburke@ psych.ryerson.ca)

If you have questions regarding your rights as a human subject and participant in this study, you may contact the Ryerson University Research Ethics Board for information: Chair, Research Ethics Board, c/o Office of the Vice President, Research and Innovation, Ryerson University, 350 Victoria Street, Toronto, ON M5B 2K3, CANADA; +1-416-979-5042, rebchair@ryerson.ca 


\section{Appendix BB: Experiment 3 Consent Forms}

RYERSON

UNIVERSITY

\author{
Ryerson University \\ Consent Agreement \\ Criminal Investigation 3
}

You are being asked to participate in a research study. Before you give your consent, it is important that you read the following information and ask as many questions as necessary to be sure you understand what you will be asked to do.

$\begin{array}{llll}\text { Investigator: } & \begin{array}{l}\text { Sara Cowan, MA } \\ \text { PhD Candidate } \\ \text { Department of Psychology }\end{array} & \text { Supervisor: } & \begin{array}{l}\text { Dr. Tara Burke } \\ \text { Associate Professor } \\ \text { Department of }\end{array} \\ \text { Psychology } & & \end{array}$

Purpose of the Study: The purpose of this study is to examine how individuals determine the guilt or innocence of a suspect in a crime. In order to study this issue, approximately 300 individuals with accounts on Amazon's Mechanical Turk website will be asked to participate in this research. Participants must be 18 years of age or older, and must be currently living in the United States or Canada.

Description of the Study: If you decide to participate in this research, you will be asked to do the following: You will read a newspaper article describing an upcoming criminal trial, answer some questions about your thoughts about the case, and fill out two personality questionnaires. Participants may choose to participate at the time and location of their choosing. It will take approximately 40 minutes to complete.

What is Experimental in this Study: None of the procedures or questionnaires used in this study are experimental in nature, in the sense that they have all been used by other researchers and found to be useful procedures and questionnaires. From a technical or procedural point of view, part of this study is considered "experimental," because by following the procedure described above, the study examines the impact of one variable (called the "independent variable") on another variable ("called the dependent variable"). You will be given more information about the independent and dependent variables in this study at the end of the study.

Risks or Discomforts: Occasionally people feel uncomfortable when answering questions that ask about attitudes toward controversial social issues (e.g., vaccination). While we do not anticipate that any of the questions you will be responding to are controversial, if any aspect of this study makes you feel uncomfortable, you may choose not to answer certain questions, or to withdraw from the study at any time without penalty. If you wish to withdraw from the study, please continue to click through the pages, but do NOT respond to any questions. This will indicate your desire to withdraw, and the researchers will delete all responses you have provided up to that time.

Benefits of the Study: We anticipate that you will benefit from this study by learning about how psychology research is conducted. When the session is over, you will be shown a debriefing 
form that will describe in detail the procedures and hypotheses of the study. Also, once we have analysed the data (toward the end of the July), you are welcome to contact the researchers by email to view the results.

Confidentiality: Your responses in this research will be anonymous; we will not ask you to provide your name. The data collected from this study will be stored confidentially for a minimum of 5 years as computer files that will be stored on password-protected computers. Only the investigator and her faculty supervisor will have access to this information. Wherever possible, participants will be identified only by a participant number assigned to you by the researchers. This number will not be used later to identify. The online questionnaire is hosted by Qualtrics, a web survey company located in the USA and as such, is subject to U.S. laws; in particular, the US Patriot Act, which allows authorities access to the records of internet service providers. This survey or questionnaire does not ask for personal identifiers or any information that may be used to identify you. However, if you choose to participate in the study, you understand that your responses to the survey questions will be stored, and can be accessed, in the USA. The security and privacy policy for the web survey company can be found at the following link: http://www.qualtrics.com/security-statement/

Incentives to Participate: Participants will be given \$1 through Amazon's Mechanical Turk as compensation for the time required to complete the study.

Voluntary Nature of Participation: Participation in this study is voluntary. If you decide to participate, you are free to withdraw your consent and to stop your participation at any time without penalty or loss of benefits to which you are allowed. Should you withdraw from the study, you will still be given $\$ 1$.

Dissemination of Results: The results of this study will form part of Sara Cowan's $\mathrm{PhD}$ dissertation. As well, results may be shared with others at scholarly meetings or as part of published papers. However, all information will be presented in aggregate form. That is, none of your individual information will be identifiable in any way. Anonymized data may be provided to other researchers for the purpose of study or verification of results; any data that is shared will NOT include the names of ANY participants.

Questions about the Study: If you have any questions about the research at any time, you may contact: Sara Cowan +1-416-979-5000 x 2190, sara.cowan@psych.ryerson.ca ; Dr. Tara Burke, +1-416-979-5000, ex.6519, tburke@psych.ryerson.ca

If you have questions regarding your rights as a human subject and participant in this study, you may contact the Ryerson University Research Ethics Board for information: Chair, Research Ethics Board, c/o Office of the Vice President, Research and Innovation, Ryerson University, 350 Victoria Street, Toronto, ON M5B 2K3, 416-979-5042, rebchair@ryerson.ca

Agreement: Clicking YES below indicates that you have read the information in this agreement and have had a chance to ask any questions you have about the study. Selecting YES also indicates that you agree to be in the study and have been told that you can change your mind and 
withdraw your consent to participate at any time.

You have been told that by signing this consent agreement you are not giving up any of your legal rights.

- YES, I would like to participate in the study

- NO, I do NOT want to participate in the study 


\section{Appendix CC: Experiment 3 Fictitious Newspaper Articles}

\section{First Nations, Seattle Condition}

\section{Seattle'Tribune \\ Seattle $52^{\circ}$ \\ Overcast \\ Detailed Forecast}

\section{Home | News | Opinion | Business | Sports | Entertainment | Life | Health | Technology | Travel | Jobs | Cars | Classifieds}

\section{Crowfoot Trial Set to Begin}

Published: Wednesday, March 16, 2016, 5:40 PM Updated: Thursday, March 17, 2016, 3:22 PM

By Kevin Kerslake - Seattle Tribune

Follow

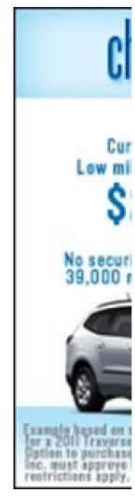

The trial of a man accused of killing 23-year-old Kendra Snow in Seattle in a robbery gone wrong nearly three years ago is set to begin next week.

Joseph Crowfoot, 27, also of Seattle is charged with second degree murder and robbery.

Snow's co-workers alerted the police when she did not show up for work on May 12, 2013. Police found her body in her apartment later that day. The apartment appeared to have been robbed. Snow's laptop computer and other valuables had been taken. None have been recovered by police.

Julia Bowling, a neighbor of Snow's reported seeing a young man leaving the apartment building the previous evening. She described him as being American Indian, with a medium build. Crowfoot is American Indian and fits the witness' description.

More V

Police found fingerprints in Snow's kitchen. These were later matched to Joseph Crowfoot. The defense intends to argue that the fingerprints were left when Crowfoot was hired to fix the sink in the apartment. The property management company has confirmed that Crowfoot fixed the sink on May 9, 2013.

The prosecution claims that Crowfoot returned later to the apartment to steal items of value. They will argue Crowfoot panicked when Snow returned home and killed her.

No DNA or fingerprint evidence was found on Snow's body.

The defense will focus on Crowfoot's alibi. During his initial interrogation, Crowfoot told police that he was at a concert at a venue downtown with friends during the time of the crime. Crowfoot's friends have been interviewed by police and, according to sources, have confirmed that he was with them on the night in question. The prosecution will likely attempt to discredit the friends' statements. The defense has not revealed what other evidence they will bring to support the alibi. 


\title{
First Nations Vancouver Condition
}

Crowfoot Trial to Begin

Page 1 of 1

Start Home Delivery | Subscriber Services | Digital Edition | Newspaper in Education | Email Alerts | Mobile Products | Contact Us

\section{Vancouver'Tribune}

Search...

\section{Home News $\mid$ Opinion | Business $\mid$ Sports $\mid$ Entertainment $\mid$ Life | Health | Technology $\mid$ Travel | Jobs |Cars | Classifieds}

\section{Crowfoot Trial Set to Begin}

Published: Wednesday, March 16, 2016, 5:40 PM Updated: Thursday, March 17, 2016, 3:22 PM

\author{
By Kevin Kerslake - Vancouver Tribune \\ Follow

The trial of a man accused of killing 23-year-old Kendra Snow in Vancouver in a robbery gone wrong nearly three years ago is set to begin next week.

Joseph Crowfoot, 27, also of Vancouver is charged with second degree murder and robbery.

Snow's co-workers alerted the police when she did not show up for work on May 12, 2013. Police found her body in her apartment later that day. The apartment appeared to have been robbed. Snow's laptop computer and other valuables had been taken. None have been recovered by police.

Sponsore

Julia Bowling, a neighbour of Snow's reported seeing a young man leaving the apartment building the previous evening. She described him as being First Nations, with a medium build.

Crowfoot is First Nations and fits the witness' description.

More V

Police found fingerprints in Snow's kitchen. These were later matched to Joseph Crowfoot. The defense intends to argue that the fingerprints were left when Crowfoot was hired to fix the sink in the apartment. The property management company has confirmed that Crowfoot fixed the sink on May 9, 2013.

The prosecution claims that Crowfoot returned later to the apartment to steal items of value. They will argue Crowfoot panicked when Snow returned home and killed her.

No DNA or fingerprint evidence was found on Snow's body.

The defense will focus on Crowfoot's alibi. During his initial interrogation, Crowfoot told police that he was at a concert at a venue downtown with friends during the time of the crime. Crowfoot's friends have been interviewed by police and, according to sources, have confirmed that he was with them on the night in question. The prosecution will likely attempt to discredit the friends' statements. The defense has not revealed what other evidence they will bring to support the alibi. 


\section{White Seattle Condition}

Campbell Trial to Begin

Page 1 of 1

Start Home Delivery | Subscriber Services | Digital Edition | Newspaper in Education | Email Alerts | Mobile Products | Contact Us

\section{Seattle'Tribune \\ Seattle $52^{\circ}$ \\ Overcast \\ Detailed Forecast}

Search...

Home News Opinion | Business | Sports | Entertainment | Life | Health | Technology | Travel| Jobs |Cars |Classifieds

\section{Campbell Trial Set to Begin}

Published: Wednesday, March 16, 2016, 5:40 PM Updated: Thursday, March 17, 2016, 3:22 PM

By Kevin Kerslake - Seattle Tribune

Follow

The trial of a man accused of killing 23-year-old Kendra Snow in Seattle in a robbery gone wrong nearly three years ago is set to begin next week.

Joseph Campbell, 27, also of Seattle is charged with second degree murder and robbery.

Snow's co-workers alerted the police when she did not show up for work on May 12, 2013. Police found her body in her apartment later that day. The apartment appeared to have been robbed. Snow's laptop computer and other valuables had been taken. None have been recovered by police.

Julia Bowling, a neighbor of Snow's reported seeing a young man leaving the apartment building the previous evening. She described him as being White, with a medium build. Campbell is White and fits the witness' description.

Police found fingerprints in Snow's kitchen. These were later matched to Joseph Campbell. The defense intends to argue that the fingerprints were left when Campbell was hired to fix the sink in the apartment. The property management company has confirmed that Campbell fixed the sink on May 9, 2013.

The prosecution claims that Campbell returned later to the apartment to steal items of value. They will argue Campbell panicked when Snow returned home and killed her.

No DNA or fingerprint evidence was found on Snow's body.

The defense will focus on Campbell's alibi. During his initial interrogation, Campbell told police that he was at a concert at a venue downtown with friends during the time of the crime. Campbell's friends have been interviewed by police and, according to sources, have confirmed that he was with them on the night in question. The prosecution will likely attempt to discredit the friends' statements. The defense has not revealed what other evidence they will bring to support the alibi. 


\section{White Vancouver Condition}

Campbell Trial to Begin

Page 1 of 1

Start Home Delivery | Subscriber Services | Digital Edition | Newspaper in Education | Email Alerts | Mobile Products | Contact Us

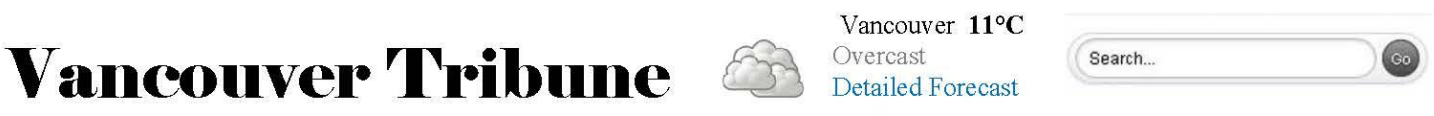
Home News Opinion | Business | Sports | Entertainment | Life | Health | Technology | Travel| Jobs |Cars |Classifieds

\section{Campbell Trial Set to Begin}

Published: Wednesday, March 16, 2016, 5:40 PM Updated: Thursday, March 17, 2016, 3:22 PM

The trial of a man accused of killing 23-year-old Kendra Snow in Vancouver in a robbery gone wrong nearly three years ago is set to begin next week.

Joseph Campbell, 27, also of Vancouver is charged with second degree murder and robbery.

Snow's co-workers alerted the police when she did not show up for work on May 12, 2013. Police found her body in her apartment later that day. The apartment appeared to have been robbed. Snow's laptop computer and other valuables had been taken. None have been recovered by police.

Julia Bowling, a neighbour of Snow's reported seeing a young man leaving the apartment building the previous evening. She described him as being White, with a medium build. Campbell is White and fits the witness' description.

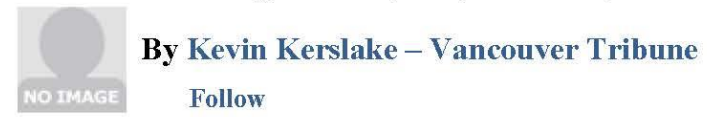

By Kevin Kerslake - Vancouver Tribune

Follow

Police found fingerprints in Snow's kitchen. These were later matched to Joseph Campbell. The defense intends to argue that the fingerprints were left when Campbell was hired to fix the sink in the apartment. The property management company has confirmed that Campbell fixed the sink on May 9, 2013.

The prosecution claims that Campbell returned later to the apartment to steal items of value. They will argue Campbell panicked when Snow returned home and killed her.

No DNA or fingerprint evidence was found on Snow's body.

The defense will focus on Campbell's alibi. During his initial interrogation, Campbell told police that he was at a concert at a venue downtown with friends during the time of the crime. Campbell's friends have been interviewed by police and, according to sources, have confirmed that he was with them on the night in question. The prosecution will likely attempt to discredit the friends' statements. The defense has not revealed what other evidence they will bring to support the alibi. 


\section{Appendix DD: Experiment 3 Questionnaire}

\section{Criminal Investigations 3 Questionnaire}

If you wish to discontinue your participation, you MUST click through the remaining pages until the end of the survey, but do NOT answer any questions. The last page will provide you with a code that you must enter onto the mTurk site to be paid.

- $\mathrm{OK}$

1. What is your age? years

2. In terms of your gender, you would self-identify as (choose all that apply):

- Female

- Male

- Trans*

- Queer

- Other, please specify

3. What is your ethnicity (choose all that apply)

- American Indian or Alaska Native

- Indigenous Canadian (First Nations, Inuit, Métis)

- Black or African American

- East Asian (e.g., Chinese, Japanese, Korean)

- Hispanic or Latino/a

- Native Hawaiian or Other Pacific Islander

- West Asian/Middle Eastern

- White

- Other, please specify

4. In what American State or Canadian Province do you currently reside?

5. How long have you lived in this American State or Canadian Province?

- Less than one year

- 1-2 years

- 3-5 years

- 6-10 years

- $11+$ years

6. How strongly do you identify as a Canadian/American?

Not at all

Somewhat

Very 
$\mathrm{O}$

$\mathrm{O}$

$\mathrm{O}$

$\mathrm{O}$

$\mathrm{O}$

$\mathrm{O}$

$\mathrm{O}$

7. How proud are you to be Canadian/American?

Not at all

Somewhat

Very
$\mathrm{O}$
$\mathrm{O}$
$\mathrm{O}$
$\mathrm{O}$
$\mathrm{O}$
$\mathrm{O}$
$\mathrm{O}$

8. How long have you been signed up as a Worker on Mechanical Turk?

- Less than 6 months

- 6 months to one year

- $1-2$ years

- 3-5 years

- 6-10 years

9. About how many HITs have you completed as a Worker on Mechanical Turk?

- Less than 10

- 11-25

- $26-50$

- $50-100$

- $101+$

\section{NEWSPAPER ARTICLES PRESENTED HERE}

10. How honest do you think the defendant is being in his description of his whereabouts on the night that Kendra Snow was killed?

Not at all

honest

$\mathrm{O}$

Somewhat

Very

honest

honest

$\mathrm{O}$

$\mathrm{O}$

11. How confident are you in your decision for the previous question?

Not at all

confident

O

O

O

O

O

$\mathrm{O}$

O

O 
12. How accurate do you think the defendant's statement regarding his whereabouts on the night Kendra Snow was killed is? In other words, how well does his statement match his actual activities on the night in question?

Not at all

accurate

$\mathrm{O}$
$\mathrm{O}$

$\mathrm{O}$
Somewhat

accurate
Very

accurate

13. How confident are you in your decision for the previous question?

Not at all

confident
$\mathrm{O}$

$\mathrm{O}$
Somewhat

confident
Very

confident

$\mathrm{O}$

$\mathrm{O}$

$\mathrm{O}$

$\mathrm{O}$

$\mathrm{O}$

14. When the defendant said that he was somewhere else when the crime was committed, his description of what he was doing at the time was probably:

- The truth; he was telling the police where he was at the time

- A lie; he was deliberately giving false information to the police

- An error; he was trying to tell the truth, but was mistaken about his whereabouts

15. Overall, how strong do you think the evidence is against the defendant?

Not at all

strong

$\mathrm{O}$
$\mathrm{O}$

$\mathrm{O}$
Somewhat

strong
Very

strong

16. Based on the evidence provided to you, how likely do you think it is that the defendant committed the crime?

Not at all

likely

$\mathrm{O}$

$\mathrm{O}$

$\mathrm{O}$

$\mathrm{O}$

$\mathrm{O}$

$\mathrm{O}$

Very

likely

likely

$\mathrm{O}$

$\mathrm{O}$

17. If you were asked to provide a verdict in the case, you would vote that the defendant is 
- Guilty

- Not Guilty

18. Why did you choose the verdict that you did? TEXT-ENTRY

19. In general, how similar do you think you are to the defendant?

Not at all

similar

$\mathrm{O}$
Somewhat

similar
Very

similar

$\mathrm{O} \quad \mathrm{O}$

$\mathrm{O}$

$\mathrm{O}$

20. In terms of personality, how similar do you think you are to the defendant?

Not at all

similar
Somewhat

similar
Very

similar
$\mathrm{O}$
$\mathrm{O}$
$\mathrm{O}$
$\mathrm{O}$
$\mathrm{O}$
$\mathrm{O}$
$\mathrm{O}$

21. In terms of activities that you enjoy, how different do you think you are to the defendant?

Not at all

similar

$\mathrm{O}$

$\mathrm{O}$

$\mathrm{O}$

Somewhat

Very

similar

similar

22. How much do you like the defendant if you met?

Not at all

Somewhat

A lot

$\mathrm{O}$

$\mathrm{O}$

$\mathrm{O}$

$\mathrm{O}$

$\mathrm{O}$

$\mathrm{O}$

$\mathrm{O}$

23. If you met the defendant, how likely is it that you would become friends?

Not at all

Somewhat

Very

likely

likely

likely 

O
$\mathrm{O}$
$\mathrm{O}$
$\mathrm{O}$
$\mathrm{O}$
$\mathrm{O}$
$\mathrm{O}$

24. How would you feel if the defendant lived next door to you? (11-POINT SCALE)
Very
Neutral
Very
negative
positive

$\mathrm{O}$

O

O

O

$\mathrm{O}$

$\mathrm{O}$

$\mathrm{O}$

25. How would you feel if the defendant was one of your co-workers at your job? (11-POINT SCALE)
Very
Neutral
Very
negative
positive
$\mathrm{O}$
O
O
O
O
O
O

26. How would you feel if the defendant was in a romantic relationship with one of your family members? (11-POINT SCALE)
Very
Neutral
Very
negative
positive
$\mathrm{O}$
$\mathrm{O}$
$\mathrm{O}$
$\mathrm{O}$
$\mathrm{O}$
$\mathrm{O}$
$\mathrm{O}$

27. Overall, you think that the defendant is: (11-POINT SCALE)
A very bad
Neither
A very
person
good nor
good
bad
person
O
O
O
$\mathrm{O}$
$\mathrm{O}$
$\mathrm{O}$
$\mathrm{O}$

28. Where did the defendant say he was at the time of the crime?

- At a concert

- At a sporting event 
- At a movie

- At home alone

- At the gym

29. A witness said she saw a young man leave the apartment building that night. She described this man as being:

- White

- First Nations

- African American

- Middle Eastern

- She did not say

30. The crime took place in:

- Seattle, WA

- Vancouver, BC

- Toronto, ON

- New York, NY

31. What is the tallest mountain in the world?

- Mount St. Helen

- Mount Everest

- Mount Washington

- Matterhorn

32. Who was the President of the United States before Barack Obama?

- George W. Bush

- Bill Clinton

- John McCain

- Jesse Ventura

33. What is the longest river in the world?

- The Okanagan River

- The Nile

- The Assiniboine River

- The Fraser River

34.. Please write below, in your own words, how you define the term $\underline{\text { ALIBI}}$. This word is pronounced like al-lee-bye.

35. My definition for the term ALIBI was:

- what I already knew about the word

- what I Googled about the word

36. Please provide any general comments you have about the case. 
37. Please indicate how you feel about members of the following groups (0-100 VISUAL ANALOG SCALE):

- African Americans

- First Nations/American Indian/Indigenous People

- Caucasian/White People

- Muslims

- Christians

- Jewish People

- Senior Citizens

- Adolescents

- Males

- Females

- Transgender Individuals

- Homosexuals

- Heterosexuals

- Bisexuals 


\section{Appendix EE: Revised Religious Life Inventory (RLI-R; Hills et al., 2005)}

Note: Original wording is provided. Italicized sections were deleted and sections in brackets were added.

\section{Extrinsic Scale}

1. The primary purpose of prayer is to gain relief and protection.

2. The church (A place of worship [e.g., church, temple, synagogue, mosque]) is most important as a place to formulate good social relationships.

3. What religion offers me most is comfort when sorrows and misfortune strike.

4. I pray chiefly because I have been taught to pray.

5. A primary reason for my interest in religion is that my church (place of worship [ e.g., mosque, synagogue, temple, church]) is a congenial social activity.

6. Occasionally I find it necessary to compromise my religious beliefs in order to protect my social and economic well-being.

7. The purpose of prayer is to secure a happy and peaceful life.

Intrinsic Scale

1. It is important for me to spend periods of time in private religious thought and meditation.

2. If not prevented by unavoidable circumstances, I attend church (my place of worship [e.g., temple, church, mosque, synagogue]).

3. I try hard to carry my religion over into all my other dealings in life.

4. The prayers I say when I am alone carry as much meaning and personal emotion as those said by me during services.

5. Quite often I have been keenly aware of the presence of God or the Divine Being.

6. I read literature about my faith or church $\{/$ temple/mosque/synagogue).

7. If I were to join a church group (at my place of worship), I would prefer to join a Bible (religious) study group rather than a social fellowship.

8. My religious beliefs are what really lie behind my whole approach to life.

9. Religion is especially important to me because it answers many questions about the meaning of life.

Quest Scale

1. As I grow and change, I expect my religion also to grow and change.

2. I am constantly questioning my religious beliefs.

3. It might be said that I value my religious doubts and uncertainties.

4. I was not very interested in religion until I began to ask questions about the meaning and purpose of my life.

5. I have been driven to ask religious questions out of a growing awareness of the tensions in my world and in my relation to my world.

6. My life experiences have led me to rethink my religious convictions.

7. There are many religious issues on which my views are still changing.

8. Questions are far more central to my religious experience than are answers.

*All items are responded to on a nine-point scale from strongly agree (1) to strongly disagree (9). 


\section{Appendix FF: Experiment 3 Non-Significant Results for Non-Legal Judgments}

Liking the defendant. Participants also rated how much they thought they would like the defendant if they met him on a 7-point Likert scale. There were no significant main or interaction effects (see Table FF1). The overall mean was $4.08(S D=1.07)$, which suggests that participants were unsure whether they would like the defendant or not if they ever met him.

Table FF1

ANOVA results for participants' ratings of how much they think they would like the defendant.

\begin{tabular}{lccc}
\hline Effect & $F$ & $p$ & $\eta_{\mathrm{p}}^{2}$ \\
\hline Defendant Race & 1.81 & .180 & .008 \\
Crime Location & 1.23 & .268 & .005 \\
Sample Location & .94 & .334 & .004 \\
Defendant Race X Crime Location & .71 & .402 & .003 \\
Defendant Race X Sample Location & .80 & .373 & .003 \\
Crime Location X Sample Location & .17 & .680 & .001 \\
Defendant Race X Crime Location X Sample Location & .34 & .563 & .001 \\
\hline
\end{tabular}

Note. All effects are $d f=1,227$

Feelings if the defendant moved in next door. Participants rated how they would feel if the defendant moved in next door on a scale from 1 (Very Negative) to 11 (Very Positive), with the midpoint labeled (Neutral). There were no significant main or interactive effects observed (see Table FF2). Overall, participants had slightly negative feelings about the prospect of the defendant moving in next door $(M=5.45, S D=1.78)$.

Feelings if defendant was a coworker. Participants also rated how they would feel if the defendant was a coworker on an 11-point Likert scale. No significant main or interactive effects were observed (see Table FF3). Overall, participants would feel slightly negative about working with the defendant $(M=5.53, S D=1.73)$. 
Table FF2

ANOVA results for participants' ratings of how they would feel if the defendant moved next door.

\begin{tabular}{lccc}
\hline Effect & $F$ & $p$ & $\eta_{\mathrm{p}}^{2}$ \\
\hline Defendant Race & 2.02 & .156 & .009 \\
Crime Location & .09 & .763 & $<.001$ \\
Sample Location & .13 & .721 & .001 \\
Defendant Race X Crime Location & .01 & .936 & $<.001$ \\
Defendant Race X Sample Location & .28 & .597 & .001 \\
Crime Location X Sample Location & 1.55 & .214 & .007 \\
Defendant Race X Crime Location X Sample Location & .001 & .980 & $<.001$ \\
\hline
\end{tabular}

Note. All effects are $d f=1,226$

Table FF3

ANOVA results for participants' ratings of how they would feel if the defendant was a coworker.

\begin{tabular}{lccc}
\hline Effect & $F$ & $p$ & $\eta_{\mathrm{p}}^{2}$ \\
\hline Defendant Race & .07 & .785 & $<.001$ \\
Crime Location & .46 & .500 & .002 \\
Sample Location & .39 & .534 & .002 \\
Defendant Race X Crime Location & .03 & .874 & $<.001$ \\
Defendant Race X Sample Location & .01 & .916 & $<.001$ \\
Crime Location X Sample Location & .19 & .667 & .001 \\
Defendant Race X Crime Location X Sample Location & .01 & .931 & $<.001$ \\
\hline
\end{tabular}

Note. All effects are $d f=1,226$

\section{Feelings if the defendant was in a romantic relationship with a family member of the}

participant. Participants also rated how they would feel if the defendant was involved in a

romantic relationship with one of their family members on an 11-point Likert scale. No

significant main or interaction effects were found (see Table FF4). In general, participants would have negative feelings about the defendant dating one of their family members $(M=4.84, S D=$ 2.19). 


\section{Table FF4}

ANOVA results for participants' feelings about the defendant dating one of their family members.

\begin{tabular}{lccc}
\hline Effect & $F$ & $p$ & $\eta_{\mathrm{p}}^{2}$ \\
\hline Defendant Race & .27 & .607 & .001 \\
Crime Location & .15 & .699 & .001 \\
Sample Location & .11 & .740 & $<.001$ \\
Defendant Race X Crime Location & .21 & .645 & .001 \\
Defendant Race X Sample Location & .05 & .821 & $<.001$ \\
Crime Location X Sample Location & .08 & .780 & $<.001$ \\
Defendant Race X Crime Location X Sample Location & .33 & .569 & .001 \\
\hline
\end{tabular}

Note. All effects are $d f=1,226$ 


\section{Appendix GG: Psychometric Analyses of the ACT and RLI-R for Experiment 3 \\ ACT Scale}

The internal consistency for the scale overall was impressive $(\alpha=.932)$. The alpha level would not increase if any of the items were deleted, though it would remain the same if any of three items were deleted. All three items had acceptable inter-item correlations $(r=.424, .444$, .483) and generally correlated well with other items (most $r>3$ ), suggesting that the items are not particularly problematic.

The Conservatism subscale showed excellent internal consistency $(\alpha=.901)$, and the alpha level would decrease if any of the items were removed. Five of the six items had item-total correlations of .7 or greater, and the sixth had an item-total correlation of .644. All inter-item correlations were .461 or greater. All items from the Conservatism subscale were retained.

The Traditionalism subscale showed very good internal consistency $(\alpha=.864)$. The alpha would increase to .883 if one of the items, a reverse scored question about whether it is desirable to "stick to the "straight and narrow," was removed. However, the item-total correlation for this item was .381 , and it correlated adequately $(r>.3)$ with all but one other item. The item is not obviously problematic and, consequently, was retained. The item-total and inter-item correlations did not show any other problems with particular items.

The Authoritarianism subscale also achieved very good internal consistency $(\alpha=.841)$. The alpha level would increase slightly to .848 if one of the items was deleted, though the itemtotal correlation for this item was .438 and it correlated well with all but one other item. Itemtotal and inter-item correlations for all other items were well within acceptable limits. All 18 items were retained for analysis.

Total possible scores on the ACT ranged from 18 to 126 . The average score was 
somewhat below the midpoint of $72(M=59.47, S D=21.41$, Range: $18-126)$. Mean scores from Experiment 1 and 2 were also slightly below the midpoint. ACT total scores were significantly higher in the American sample $(M=64.23, S D=24.35)$ than in the Canadian sample $(M=55.46$, $S D=17.70), t(189.71)=3.10, p=.002, d=0.42$, though scores for both groups were below the midpoint. A significant Levene's Test also indicates that there is significantly higher variability in ACT total scores in the American sample than in the Canadian sample $(p<.001)$.

Total possible scores on the subscales ranged from 6 to 42 . The average score for the Conservatism subscale was below the midpoint of $24(M=20.30, S D=8.16$, Range: $6-42)$. As with the ACT total scores, scores on the Conservatism subscale were significantly higher and showed significantly more variability in the American sample $(M=21.93, S D=9.39)$ than in the Canadian sample $(M=18.93, S D=6.71), t(187.59)=2.76, p=.006, d=0.33$, Levene's test $p<$ .001. The same pattern was observed for the Traditionalism subscale. The average score across the sample was below the midpoint $(M=17.15, S D=8.40$, Range: $6-42)$, and scores from the American sample $(M=19.02, S D=9.24)$ were significantly higher and more variable than the scores for the Canadian sample $(M=15.59, S D=7.31), t(200.18)=3.12, p=.002, d=0.42$, Levene's test $p=.003$. For the Authoritarianism subscale, the average score was only slightly below the midpoint $(M=22.06, S D=7.85$, Range: 6-42). Again, higher scores were found for the American sample $(M=23.29, S D=8.30)$ than the Canadian sample $(M=21.04, S D=7.34)$, $t(233)=2.21, p=.028, d=0.29$, though the variance was not significantly different between the samples for these scores.

\section{RLI-R}

The Extrinsic subscale had adequate internal consistency $\left(\alpha=.757, N=102^{15}\right)$, which

\footnotetext{
${ }^{15}$ For these analyses, SPSS, version 22, excludes participants listwise, so reliabilities are computed based only on participants who responded to all of the items.
} 
was similar to the figures provided by Hills et al., $(2005 ; .79, .76)$. Examination of the inter-item correlations shows that all items correlate well with some other items and poorly with other items. However, the item-total correlations are acceptable (all $r>.360$ ) and the alpha would decrease if any of the items were removed. Based on these findings, it is plausible that the scale is measuring more than one subtype of extrinsic religious orientation. Further examination of this issue, however, is beyond the scope of the current work and is not necessary for investigating the hypotheses of interest. The average response for the Extrinsic orientation subscale was 5.33 (SD $=1.51$, Range: $1-9)$. No significant differences were observed between the Canadian $(M=5.58$, $S D=1.60, n=50)$ and American samples $(M=5.39, S D=1.61, n=70), t(118)=.64, p=.526$, $d=0.23$.

The Intrinsic orientation showed exceptional internal consistency $(\alpha=.950, N=109)$. This figure is very similar to the alphas reported by Hills et al. (2005; .93, .93). Item-total correlations were all excellent (all $r>.695$ ), all items correlated well with all other items (all $r>$ $.548)$, and the alpha would decrease or remain the same if any of the items were deleted. The average mean score on the Intrinsic orientation subscale was $4.63(S D=2.33$, Range: $1-9)$. The scores on this subscale were not normally distributed, so an independent-samples median test was computed to compare the results of the Canadian $(M d n=5.56)$ and American $(M d n=4.67)$ samples. This test was not significant, $p=.155$.

The Quest orientation subscale had very good internal consistency $(\alpha=.853, N=119)$. This is also similar to Hills et al.'s (2005) figures $(.77, .83)$. Item-total correlations are all acceptable (all $r>.465$ ), the alpha would decrease if any of the items were removed, and there are very few inter-item correlations lower than .3 in the correlation matrix. The average mean score on the Quest subscale was 4.90 ( $S D=1.75$, Range: 1-9). No significant differences were 
observed between the Canadian $(M=4.92, S D=1.73)$ and American samples $(M=5.11, S D=$ $1.90), t(136)=-.61, p=.540, d=-0.10$. 


\section{Appendix HH: Inferential Tests Based on Qualitative Responses in Experiment 3}

It is also possible that participants' legal judgments differed based on their understanding of the term "alibi." In particular, it is of interest to investigate whether participants who indicated that an alibi is a narrative describing a suspect's whereabouts during the time of a crime (code 1) and participants who indicated that an alibi is the evidence that supports a statement differed on these judgments (code 2). Across both samples, 111 participants (47.7\%) were in the code 1 group and 67 (28.5\%) were in the code 5 group. The code 1 group comprised 65 Canadians and 47 Americans, and the code 5 group comprised 36 Canadians and 31 Americans. Sample Location was not hypothesized to interact with any differences between the code 1 and code 5 groups, so this variable was not included in the analyses. Independent-samples $t$-tests did not show any significant differences between the two groups (see Table HH1). Additionally, the association between group membership and verdict choice was not significant, $\chi^{2}(1)=.20, p=$ $.803, \varphi=-.033$. These results suggest that participants' understanding of the term "alibi" is unrelated to their legal judgments.

Table HH1

Comparison of legal judgments between participants who provided correct definitions and participants who emphasized an alibi as evidence.

\begin{tabular}{lcccccc}
\hline Outcome & $d f$ & $t$ & $p$ & $d$ & $M 1(S D)$ & $M 2(S D)$ \\
\hline Defendant honesty & 176 & -.19 & .853 & -0.03 & $4.60(1.35)$ & $4.64(1.27)$ \\
Confidence in defendant honesty & 177 & -1.29 & .199 & -0.20 & $3.98(1.76)$ & $4.33(1.71)$ \\
Alibi accuracy & 177 & -.31 & .760 & -0.05 & $4.79(1.43)$ & $4.87(1.62)$ \\
Confidence in alibi accuracy & 176 & -1.26 & .211 & -0.20 & $4.30(1.82)$ & $4.64(1.69)$ \\
$\begin{array}{l}\text { Strength of evidence against the } \\
\quad\end{array}$ & 177 & .22 & .830 & 0.03 & $2.73(1.36)$ & $2.69(1.38)$ \\
$\quad$ defendant & 117 & .95 & .343 & 0.15 & $3.07(1.26)$ & $2.88(1.37)$ \\
\hline
\end{tabular}

Note. M1 refers to participants who referred to the alibi as a narrative regarding a suspect's whereabouts at the time of a crime, whereas M2 refers to the participants whose responses emphasized the evidence supporting a statement. 


\section{Appendix II: Experiment 3 Debrief Form}

\section{Debriefing}

The social psychology study that you just participated in examines the factors associated with the evaluation of an alibi. An alibi is the explanation a suspect gives to the police regarding his or her whereabouts when a crime is committed. The Innocence Project

(http://www.innocenceproject.org/), an organization that helps the wrongly convicted, found that approximately $25 \%$ of the individuals they helped to exonerate had presented an alibi before being convicted, but it wasn't believed. Previous research has demonstrated that alibi evidence (that is ultimately true) is often considered 'weak' by the justice system and therefore ignored (Burke, Turtle and Olson, 2007). As well, The Innocence Project shows that members of visible minority or marginalized groups are over-represented within the justice system. In Canada, First Nations people make up approximately $4 \%$ of the adult population, but represent $18-20 \%$ of incarcerated adults.

A lot of studies have focused on the strength of corroborating evidence (i.e. evidence that confirms one's story) to explain why some alibis are believed, while others are not. For example, an alibi that is confirmed by a stranger's testimony and physical evidence that would be hard to fabricate will be believed more often than an alibi corroborated by a relative of the suspect with no physical evidence to back it up. Other research shows that sometimes we are more accepting or forgiving of people we feel are similar to us (an 'in-group' bias) compared to people we perceive as being different from us (an 'out group'). In this study, we were interested in whether an in-group bias and the suspect's racial/ethnic group influenced whether the alibi was believed and whether people thought the suspect was guilty. To accomplish this, we created four different versions of the article that you read. Most of the information about the case was the same, but the defendant in the case was either a (a) White Canadian, (b) First Nations Canadian, (c) White American, or (d) Native American.

Our first hypothesis was participants would give more favourable ratings (e.g., higher belief in the defendant's alibi, lower belief in his guilt) if the defendant was from the same country as them. In other words, we expected Canadians to treat a Canadian defendant more favourably than an American defendant, and Americans to treat an American defendant more favourably than a Canadian defendant.

Our second hypothesis was that alibis provided by First Nations Canadians and Native Americans would be rated as less believable and the suspect would be seen as more likely to be guilty than when the alibi was provided by a White Canadian or American.

The results will allow us to see if the in-group bias and the racial/ethnic identity of the alibi provider have an influence on juror decisions of guilt and alibi believability. This information may allow us to help the justice system evaluate and use alibi information more appropriately.

If you know of others who will be participating in this experiment, please refrain from discussing it with them. We do not want our future participants to be aware of the procedures and expected findings. 
Thank you for participating in our research. If you have any questions about this study, or would like to receive a copy of the consent form, this debriefing form, or a report on the results at the end of the study, please contact:

Sara Cowan, MA

sara.cowan@psych.ryerson.ca

416-979-5000 x2190
Tara Burke, Ph.D.

tburke@psych.ryerson.ca

416-979-5000 x6519 


\section{References}

Adorno, T. W., Frenkel-Brunswik, E., Levinson, D. J., \& Nevitt, S. R. (1950). The authoritarian personality. Oxford, England: Harpers.

Akehurst, L., Köhnken, G., Vrij, A., \& Bull, R. (1996). Lay persons' and police officers' beliefs regarding deceptive behavior. Applied Cognitive Psychology, 10, 461-471. doi: 10.1002/(SICI)1099-0720(199612)10:6

Allison, M., \& Brimacombe, C. A. E. (2010). Alibi believability: The effect of prior convictions and judicial instructions. Journal of Applied Social Psychology, 40, 1054-1084. doi: 10.1111/j.1559-1816.2010.00610.x

Allison, M., Jung, S., Sweeney, L, \& Culhane, S. E. (2013). The impact of illegal alibi activities, corroborator involvement, and corroborator certainty on mock juror perceptions. Psychiatry, Psychology, and Law, 20. doi: 10.1080/13218719.2013.803275

Allison, M., Mathews, K. R., \& Michael, S. W. (2012). Alibi believability: The impact of salacious alibi activities. Social Behavior and Personality, 40, 605-612. doi: 10.2224/sbp.2012.40.4.605

Allison, M., Mathews, K. R., Michael, S. W., \& Overman, A. A. (2011). Brief report: Narrative qualities and perceptions of generated alibis. North American Journal of Psychology, 13, 359-365.

Allport, G. W. (1954). The nature of prejudice. Reading, MA: Addison-Wesley.

Altemeyer, B. (1981). Right-wing authoritarianism. The University of Manitoba Press.

Anderson, R. C., \& Pichert, J. W. (1978). Recall of previously unrecallable information following a shift in perspective. Journal of Verbal Learning and Verbal Behavior, 17, 112.

Ask, K., \& Granhag, P. A. (2005). Motivational sources of confirmation bias in criminal investigations: The need for cognitive closure. Journal of Investigative Psychology and Offender Profiling, 2, 43-63. http://dx.doi.org/10.1002/jip.19

Aune, R. K. \& Waters, L. L. (1994). Cultural differences in deception: Motivations to deceive in Samoans and North Americans. International Journal of Intercultural Relations, 18, 159172. doi: 10.1016/0147-1767(94)90026-4

Austin, W., Walster, E., \& Utne, M. K. (1976). Equity and the law: The effect of a harmdoer's "suffering in the act" on liking and assigned punishment. In L. Berkowitz \& E. Walster (Eds.), Advances in experimental social psychology (Vol. 9, pp. 163-190). Orlando, FL: Academic Press. 
Baddeley, A .D., \& Hitch, G. (1974). Working memory. In G.H. Bower (Ed.), The psychology of learning and motivation: Advances in research and theory (Vol. 8, pp. 47-89). New York: Academic Press.

Batson, C. D., Kobrynowicz, D., Dinnerstein, J., L., Kampf, H. C., \& Wilson, A. D. (1997). In a very different voice: Unmasking moral hypocrisy. Journal of Personality and Social Psychology, 72, 1335-1348. http://dx.doi.org/10.1037/0022-3514.72.6.1335

Batson, C. D., Schoenrade, P., \& Ventis, W. L. (1993). Religion and the individual. New York: Oxford University Press.

Benjamin, A. J. (2006). The relationship between Right-Wing Authoritarianism and attitudes toward violence: Further validation of the Attitudes Toward Violence Scale. Social Behavior and Personality, 34, 923-926. doi: 10.2224/sbp.2006.34.8.9

Berinsky, A. J., Huber, G. A., \& Lenz, G. S. (2012). Evaluating online labor markets for experimental research: Amazon.com's mechanical turk. Political Analysis, 20, 351-368. doi: 10.1093/pan/mpr057

Berry, D. S., \& Zebrowitz-McArthur, L. A. (1988). What's in a face? Facial maturity and the attribution of legal responsibility. Personality and Social Psychology Bulletin, 14, 23-33. doi: $10.1177 / 014616728$

Bjork, R. A., Dunlosky, J., \& Kornell, N. (2013). Self-regulated learning: Beliefs, techniques, and illusions. Annual Review of Psychology, 64, 417-444. doi: 10.1146/annurev-psych113011-143823

Bond, C. F., \& DePaulo, B. M. (2006). Accuracy of deception judgments. Personality and Social Psychology Review, 10, 214-234. doi: 10.1207/s15327957pspr1003_2

Boyce, M. A., Beaudry, J. L., \& Lindsay, R. C. L. (2007). Belief of eyewitness identification evidence. In R. C. L. Lindsay, D. F. Ross, J. D. Read, \& M. P. Toglia (Eds.), The handbook of eyewitness psychology (Vol. 2): Memory for people (pp. 201-225). Mahwah, NJ: Lawrence Erlbaum and Associates.

Brehm, S. S., Kassin, S., Fein, S., \& Burke, T. M. (2008). Social psychology (First Canadian Edition). Boston, MA: Houghton Mifflin Company.

Brewer, W. F., \& Nakamura, G. V. (1984). The nature and functions of schemas. In R. S. Wyer, Jr., \& T. K. Srall (Eds.), Handbook of social cognition (Vol. 1, pp. 119-160). Hillsdale, NJ: Erlbaum.

Brigham, J. C., Maass, A., Snyder, L. D., \& Spaulding, K. (1982). Accuracy of eyewitness identification in a field setting. Journal of Personality and Social Psychology, 42, 673681. doi: 10.1111/j.1559-1816.1985.tb00897.x 
Bryan, C. J., Dweck, C. S., Ross, L., Kay, A. C., \& Mislavsky, N. O. (2009). Political mindset: Effects of schema priming on liberal-conservative political positions. Journal of Experimental Social Psychology, 45, 890-895. doi: 10.1016/j.jesp.2009.04.007

Bucchianeri, M. M., Eisenberg, M. E., \& Neumark-Sztainer, D. (2013). Weightism, racism, classism, and sexism: Shared forms of harassment in adolescents. Journal of Adolescent Health, 53, 47-53. doi: 10.1016/j.jadohealth.2013.01.006

Buckner, R. L. (2000). Neuroimaging of memory. In M. S. Gazzaniga (Ed.), The new cognitive neurosciences (2nd Ed., pp. 817-828). Cambridge, MA: MIT Press.

Buhrmester, M., Kwang, T., \& Gosling, S. D. Amazon's mechanical turk: A new source of inexpensive, yet high-quality data? Perspectives on Psychological Science, 6, 3-5. doi: $10.1177 / 1745691610393980$

Bull, R. \& Rumsey, N. (1988). The social psychology of facial appearance. Berlin: Springer.

Burke, T. M. \& Marion, S. B. (2012). Alibi witnesses. In B. L. Cutler (Ed.). Conviction of the innocent: Lessons of psychological research (pp. 239-256). Washington, DC: APA Press.

Burke, T. M., Turtle, J. W., \& Olson, E. A. (2007). Alibis in criminal investigations and trials. In M. P. Toglia, J. D. Read, \& R. C. L. Lindsay (Eds.), The handbook of eyewitness psychology, Vol 1: Memory for events (pp. 157-174). Mahwah, NJ: Lawrench Erlhaum Associates Publishers.

Burt, C. D. B., Kemp, S., \& Conway, M. (2004). Memory for true and false autobiographical event descriptions. Memory, 12, 545-552.2004-16159-00110.1080/09658210344000071

Charman, S., Cahill, S. B., Leins, D., \& Carol, R. N. (2010). Investigating the ability of innocent suspects to generate accurate alibis. Paper presented at the 2010 American PsychologyLaw Society Conference, Vancouver, BC, CA.

City of Toronto (n.d.). Diversity - Toronto Facts - Your City. Retrieved 2016, May 8 from: http://www1.toronto.ca/wps/portal/contentonly?vgnextoid=dbe867b42d853410VgnVCM 10000071d60f89RCRD

Collisson, B., \& Howell, J. L. (2014). The liking-similarity effect: Perceptions of similarity as a function of liking. The Journal of Social Psychology, 154, 384-400. http://dx.doi.org/10.1080/00224545.2014.914882

Corsini, R. J. (1999). Dictionary of psychology. Philadephia, PA: Brunner/Mazel.

Cowan, S. (2013). The full picture: Can the existing deception detection literature really be generalized to real-world lying? Unpublished comprehensive paper. Ryerson University. 
Cowan, S. (2012). What you say matters: The influence of alibi content on memory and forensically relevant judgments. Unpublished master's thesis, Ryerson University.

Cowan, S., Zannella, L., Marion, S. B., \& Burke, T. M. (June, 2015). It does matter if you're black or white: The effects of race and SES on evaluations of an alibi. Paper presented at the Society for Applied Research on Memory and Cognition conference, Victoria, BC, Canada.

Cruz, M. (2015, December 15). Who is Gregory Allen? Steven Avery \& this man saw their fates indelibly tied in 1985. Bustle. Retrieved from http://www.bustle.com/articles/132803who-is-gregory-allen-steven-avery-this-man-saw-their-fates-indelibly-tied-in-1985

Culhane, S. E. (2005). Changing your alibi: Current law enforcement, future law enforcement, and layperson beliefs and behaviors. Ph. D. dissertation, University of Texas at El Paso.

Culhane, S. E., \& Hosch, H. M. (2012). Changed alibis: Current law enforcement, future law enforcement, and layperson reactions. Criminal Justice and Behavior, 39, 958-977. doi: $10.1177 / 0093854812438185$

Culhane, S. E., \& Hosch, H. M. (2004). An alibi witness' influence on mock jurors' verdicts. Journal of Applied Social Psychology, 34, 1604-1616. doi: 10.1111/j.15591816.2004.tb02789.x

Culhane, S. E., Kehn, A., Horgan, A., Meissner, C. A., Hosch, H. M., \& Wodahl, E. J. (2013). Generation and detection of true and false alibi statements. Psychiatry, Psychology and Law, 20, 619-638. http://dx.doi.org/10.1080/13218719.2012.729018

Death Penalty Information Center (2013a). States with and without the death penalty. Retrieved 5 December, 2013 from http://www.deathpenaltyinfo.org/states-and-without-deathpenalty

Death Penalty Information Center (2013b). Death penalty in flux. Retrieved 5 December, 2013 from http://www.deathpenaltyinfo.org/death-penalty-flux

Deffenbacher, K. A., Bornstein, B. H., McGorty, E. K., \& Penrod, S. D. (2008). Forgetting the once-seen face: Estimating the strength of an eyewitness's memory representation. Journal of Experimental Psychology: Applied, 14, 139-150. doi: 10.1037/1076898X.14.2.139

Devenport, J. L., Kimbrough, C. D., \& Cutler, B. L. (2009). Effectiveness of traditional safeguards against erroneous conviction arising from mistaken eyewitness identification. In B. L. Cutler (Ed.) Expert testimony on the psychology of eyewitness identification (pp. 51-68). Oxford Scholarship Online. doi: 10.1093/acprof:oso/9780195331974.003.003

Devenport, J. L., Penrod, S. D., \& Cutler, B. L. (1997). Eyewitness identification evidence: Evaluating commonsense evaluations. Psychology, Public Policy \& Law, 3, 338-361. 
Dictionary.com (2015). Salacious: Define salacious at dictionary.com. Retrieved 2015, December 17 from http://dictionary.reference.com/browse/salacious

Dion, K., Berscheid, E., \& Walster, E. (1972). What is beautiful is good. Journal of Personality and Social Psychology, 24, 285-290. doi: 10.1037/h0033731

Dixon, J. C., \& Rosenbaum, M. S. (2004). Nice to know you? Testing contact, cultural, and group threat theories of anti-Black and anti-Hispanic stereotypes. Social Science Quarterly, 85, 257-280. doi: 10.1111/j.0038-4941.2004.08502003.x

Donakowski, D. W., \& Esses, V. M. (1996). Native Canadians, First Nations, or Aboriginals: The effect of labels on attitudes toward native peoples. Canadian Journal of Behavioural Science/Review canadienne des sciences du comportement, 28, 86-91. doi: 10.1037/0008400X.28.2.86

Downs, A. C. \& Lyons, P. M. (1991). Natural observations of the links between attractiveness and initial legal judgments. Personality and Social Psychology Bulletin, 17, 541-547. doi: $10.1177 / 0146167291175009$

Duckitt, J. (1989). Authoritarianism and group identification: A new view of an old construct. Political Psychology, 10, 63-84. http://dx.doi.org/10.2307/3791588

Duckitt, J., Bizumac, B., Krauss, S. W., \& Heled, E. (2010). A tripartite approach to right-wing authoritarianism: The authoritarianism-conservatism-traditionalism model. Political Psychology, 31, 685-715. doi: 10.1111/j.1467-9221.2010.00781.x

Duckitt, J., \& Fisher, K. (2003). The impact of social threat on world view and ideological attitudes. Political Psychology, 24, 199-222. http://dx.doi.org/10.1111/0162-895X.00322

Duhaime, L. (n.d.). Alibi. Duhaime legal dictionary. Retrieved 2011, May 13, from http://www.duhaime.org/LegalDictionary/A/Alibi.aspx

Dysart, J. E. \& Strange, D. (2012). Beliefs about alibis and alibi investigations: A survey of law enforcement. Psychology, Crime, \& Law, 18, 11-25. doi: 10.1080/1068316X.2011.562867

Ebbinghaus, H. (1885/1964). Memory: A contribution to experimental psychology. New York: Dover.

Eberhardt, J. L., Davies, P. G., Purdie-Vaughns, V. J., \& Johnson, S. L. (2006). Looking deathworthy: Perceived stereotypicality of black defendants predicts capital-sentencing outcomes. Psychological science, 17, 383-386. doi: 10.1111/j.1467-9280.2006.01716.x

Efran, M. G. (1974). The effect of physical appearance on the judgment of guilt, interpersonal attraction, and severity of recommended punishment in a simulated jury task. Journal of 
Research in Personality, 8, 45-54. doi: 10.1016/0092-6566(74)90044-0

El-Sibaey, S. (2009). Believe me, believe me not: Investigating the possibility of a dual standard in the evaluation of alibi and eyewitness evidence. Unpublished master's thesis, Ryerson University.

Faul, F., Erdfelder, E., Lang, A.-G., \& Buchner, A. (2007). G*Power 3: A flexible statistical power analysis program for the social, behavioral, and biomedical sciences. Behavior Research Methods, 39, 175-191.

Fawcett, C. A., \& Markson, L. (2010). Similarity predicts liking in 3-year-old children. Journal of Experimental Child Psychology, 105, 345-358.

http://dx.doi.org/10.1016/j.jecp.2009.12.002

Findley, K. (2011, June 28). Confirmation bias in legal settings: A panel. Panel discussion at the Society for Applied Research in Memory and Cognition IX bi-annual conference, New York, NY.

First Nations and Indigenous Studies (2009). Terminology. Retrieved from http://indigenousfoundations.arts.ubc.ca/home/identity/terminology.html

France, L. R. (2016, January 7). 5 things to know about 'Making a Murderer.' $C N N$. Retrieved from: http://www.cnn.com/2016/01/07/entertainment/making-a-murderer-things-toknow-feat/

Franko, D. L., Cousineau, T. M., Rodgers, R. F., Roehrig, J. P., \& Hoffman, J. A. (2013). Socialcognitive correlates of fruit and vegetable consumption in minority and non-minority youth. Journal of Nutrition Education and Behavior, 45, 96-101.

http://dx.doi.org/10.1016/j.jneb.2011.11.006

Garrett, B. L. (2011). Convicting the innocent: Where criminal prosecutions go wrong. Cambridge, MA: Harvard University Press.

Geiselman, R. E., \& Fisher, R. P. (1989). The cognitive interview technique for victims and witnesses of crime. In D. C. Raskin (Ed.), Psychological methods in criminal investigation and evidence (pp. 191-215). New York: Springer Publishing Co.

Gilligan, C. (1977). In a different voice: Women's conceptions of self and morality. Harvard Educational Review, 47, 481-517. http://dx.doi.org.ezproxy.lib.ryerson.ca/10.17763/haer.47.4.g6167429416hg510

Glass, G. V., Peckham, P. D., \& Sanders, J. R. (1972). Consequences of failure to meet assumptions underlying the fixed effects analyses of variance and covariance. Review of Educational Research, 42, 237-288. doi: 10.3102/00346543042003237

Global Deception Research Team (2006). A world of lies. Journal of Cross-Cultural 
Psychology, 37, 60-74. doi: 10.1037/0022-3514.54.2.193

Gordon, R. A., Bindrinm, T. A., McNicholas, M. L., \& Walden, T. L. (1988). Perceptions of blue-collar and white-collar crime: The effect of defendant race on simulated juror decisions. Journal of Social Psychology, 128, 191-197. doi: 10.1080/00224545.1988.9711362

Greenberg, M. S., Westcott, D. R., \& Bailey, S. E. (1998). When believing is seeing: The effect of scripts on eyewitness memory. Law and Human Behavior, 22, 685-694. doi: 10.1023/A:1025758807624

Greene, E. (1988). Judge's instruction on eyewitness testimony: Evaluation and revision. Journal of Applied Social Psychology, 18, 252-276.

Greenwald, A. G., \& Gillmore, G. M. (1997). Grading leniency is a removable contaminant of student ratings. American Psychologist, 52, 1209-1217.

Gross, S. R. (2006). Souter passant, Scalia rampant: Combat in the Marsh. Michigan Law Review, 105, 67-72.

Gross, S. R. (1996). The risks of death: Why erroneous convictions are common in capital cases (Symposium: The New York death penalty in context). Buffalo Law Review, 44, 469-500.

Gross, S. R., Jacoby, S., Matheson, D., Montgomery, N., \& Patel, S., (2005). Exonerations in the United States: 1989 through 2003. Journal of Criminal Law and Criminology, 95, 523560.

Grusec, J. E., Kuczynski, L., Rushton, J. P., \& Simutis, Z. M. (1978). Modeling, direct instruction, and attributions: Effects on altruism. Developmental Psychology, 14, 51-57. http://dx.doi.org/10.1037/0012-1649.14.1.51

Grusec, J. E., \& Redler, E. (1980). Attribution, reinforcement, and altruism: A developmental analysis. Developmental Psychology, 16, 525-534. http://dx.doi.org/10.1037/00121649.16.5.525

Guilford, J. P. (1954). Psychometric methods (2nd ed.). New York: McGraw-Hill.

Harmon, T. R. (2001). Predictors of miscarriages of justice in capital cases. Justice Quarterly, 18, 949-968. doi: 10.1080/07418820100095141

Hives, F. II (2016). Confidence to quit: The impact of self-efficacy on substance abusers' motivation to change. Unpublished doctoral dissertation, John F. Kennedy University.

Hodson, G., Hooper, H., Dovidio, J. F., \& Gaertner, S. L. (2005). Aversive racism in Britain: The use of inadmissible evidence in legal decisions. European Journal of Social Psychology, 35, 437-448. doi: 10.1002/ejsp.261 
Hosch, H. M., Culhane, S. E., Jolly, K. W., Chavez, R. M., \& Shaw, L. H. (2011). Effects of an alibi witness's relationship to the defendant on mock jurors' judgments. Law and Human Behavior, 35, 127-142. doi: 10.1007/s10979-010-9225-5

Hosch, H. M., Culhane, S. E., \& Hawley, L. R. (2005). Effects of an alibi witness's relationship to the defendant on much jurors' judgments. Unpublished manuscript.

Hourihan, K. L., Benjamin, A. S., \& Liu, X. (2012). A cross-race effect in metamemory: Predictions of face recognition are more accurate for members of our own race. Journal of Applied Research in Memory and Cognition, 1, 158-162.

Huff, R. C., Rattner, A., \& Sagarin, E. (1986). Guilty until proven innocent: Wrongful conviction and public policy. Crime and Delinquincy, 32, 518-544. doi: $10.1177 / 0011128786032004007$

Inbau, F. E., Reid, J. E., Buckley, J. P., \& Jayne, B. C. (2011). Criminal interrogation and confession $\left(5^{\text {th }}\right.$ Edition). Sudbury, MA: Jones \& Bartlett Learning.

Jackiw, L. B., Arbuthnott, K. D., Pfeifer, J. E., Marcon, J. L., \& Meissner, C. A. (2008). Examining the cross-race effect in lineup identification using Caucasian and First Nations samples. Canadian Journal of Behavioural Science/Revue canadienne des sciences du comportement, 40, 52-57. http://dx.doi.org/10.1037/0008-400x.40.1.52

Jones, C. S., \& Kaplan, M. F. (2003). The effects of racially stereotypical crimes on juror decision-making and information processing strategies. Basic and Applied Social Psychology, 25, 1-13. doi: 10.1207/S15324834BASP2501_1

Jones, E. E. \& Davis, K. E. (1965). From acts to dispositions: The attribution process in person perception. Advances in Experimental Psychology, 2, 219-266. doi: 10.1016/00221031(67)90034-0

Jones, E. E., \& Harris, V. A. (1967). The attribution of attitudes. Journal of Experimental Social Psychology, 3, 1-24. http://dx.doi.org/10.1016/0022-1031(67)90034-0

Jugert, P., \& Duckitt, J. (2009). A motivational model of authoritarianism: Integrating personal and situational determinants. Political Psychology, 30, 693-719. http://dx.doi.org/10.1111/j.1467-9221.2009.00722.x

Jung, S., Allison, M., \& Bohn, L. (2013). Legal decision-making on crimes involving an alibi. Applied Psychology in Criminal Justice, 9, 45-58.

Kassam, K. S., Gilbert, D. T., Swencionis, J. K., \& Wilson, T. D. (2009). Misconceptions of memory: The Scooter Libby effect. Psychological Science, 20, 551-552. doi: 10.1111/j.1467-9280.2009.02334.x

Kassin, S. M., Goldstein, C. C., \& Savitsky, K. (2003). Behavioral confirmation in the 
interrogation room: On the dangers of presuming guilt. Law and Human Behavior, 27, 187-203. doi: 10.1023/A:1022599230598

Kassin, S. M., \& Gudjonsson, G. H. (2004). The psychology of confessions: A review of the literature and issues. Psychological Science in the Public Interest, 5, 33-67. doi: 10.1111/j.1529-1006.2004.00016.x

Kassin, S. M., Rigby, S., \& Castillo, S. R. (1991). The accuracy-confidence correlation in eyewitness testimony: Limits and extensions of the retrospective self-awareness effect. Journal of Personality and Social Psychology, 61, 698-707. http://dx.doi.org/10.1037/0022-3514.61.5.698

Kelley, H. H. (1972). Attribution in social interaction. In: E. E. Jones, D. E. Kanouse, H. H. Kelley, R. E. Nisbett, S. Valins, \& B. Weiner (Eds.), Attribution: Perceiving the causes of behavior (pp. 1-26). Morristown, NJ: General Learning Press.

Kerr, N. L., \& Kurtz, S. T. (1977). Effects of a victim's suffering and respectability on mock juror judgments: Further evidence on the just world theory. Representative Research in Social Psychology, 8, 42-56.

Kertscher, T. (2003, December 10). Man linked to rape had long criminal history. Milwaukee Journal Sentinel, pp. 1B, 7B.

Kohlberg, L. (1973). The claim to moral adequacy of a highest stage of moral judgment. Journal of Philosophy, 70, 630-646. doi: 10.2307/2025030

Krafka, C., \& Penrod, S. (1985). Reinstatement of context in a field experiment on eyewitness identification. Journal of Personality and Social Psychology, 48, 58-69. doi:

10.1037/0022-3514.49.1.58

Landy, D. \& Aronson, E. (1969). The influence of the character of the criminal and his victim on the decisions of simulated jurors. Journal of Experimental Social Psychology, 5, 141-152. doi: 10.1016/0022-1031(69)90043-2

Leo, R. A. (2008). Police interrogation and American justice. Cambridge, MA: Harvard University Press.

Liebman, J., Fagan, J., \& West, V. (2000). A broken system: Error rates in capital cases, 19731995. Retrieved 2013, November 12 from http://www2.law.columbia.edu/instructionalservices/liebman/

Lindsay, R. C. L., Wells, G. L., \& Rumpel, C. M. (1981). Can people detecteyewitnessidentification accuracy within and across situations? Journal of Applied Psychology, 66, 79-89. http://dx.doi.org/10.1037/0021-9010.66.1.79

Literary Ramblings (2016, January 14). Making a Murderer: Why Steven Avery's guilt or innocence is irrelevant. Retrieved from: http://www.literaryramblings.com/making-a- 
murderer-why-steven-averys-guilt-or-innocence-is-irrelevant

Loftus, E. F., Miller, D. G., \& Burns, H. J. (1978). Semantic integration of verbal information into a visual memory. Journal of Experimental Psychology: Human Learning and Memory, 4, 19-31. doi: 10.1037/0278-7393.4.1.19

Loftus, E. F. \& Palmer, J. C. (1974). Reconstruction of automobile destruction: An example of the interaction between language and memory. Journal of Verbal Learning and Verbal Behavior, 13, 585-589. doi: 10.1016/S0022-5371(74)80011-3

Lunney, G. H. (1970). Using analysis of variance with a dichotomous dependent variable: An empirical study. Journal of Educational Measurement, 7, 263-269. doi: 10.1111/j.17453984.1970.tb00727.x

MacLean, C. L., Brimacombe, C. A. E., Allison, M., Dahl, L. C., \& Kadlec, H. (2011). Postidentification feedback effects: Investigators and evaluators. Applied Cognitive Psychology, 25, 739-752. doi: 10.1007/s10979-006-9012-5

MacPhee, D., Farro, D., \& Canetto, S. S. (2014). Academic self-efficacy and performance of underrepresented STEM majors: Gender, ethnic, and social class patterns. Analyses of Social Issues and Public Policy (ASAP), 13, 347-369. http://dx.doi.org/10.1111/asap.12033

Madon, S., Guyll, M., Aboufadel, K., Montiel, E., Smith, A., \& Jussim, L. (2001). Ethnic and national stereotypes: The Princeton trilogy revisited and revised. Personality and Social Psychology Bulletin, 27, 996-1010. doi: 10.1177/0146167201278007

Maeder, E. M., \& Dempsey, J. L. (2013). A likely story? The influence of type of alibi and defendant gender on juror decision-making. Psychiatry, Psychology and Law, 20, 543552. doi: 10.1080/13218719.2012.727066

Makin, K. (2002, April 26). Top court orders new trial for man. Globe and Mail. Retrieved from http://www.theglobeandmail.com/news/national/top-court-orders-new-trial-forman/article22395854/

Marion, S. B. (2014). When do people lie, for whom, and why? Altruistic lying in an alibi corroboration context. Unpublished doctoral dissertation, Ryerson University.

Marion, S. B., \& Burke, T. M. (2013). False alibi corroboration: Witnesses lie for suspects who seem innocent, whether they like them or not. Law and Human Behavior, 37, 136-143. http://dx.doi.org/10.1037/lhb0000021

Marion, S. B., Cowan, S., Bowling, P., El-Sibaey, S., \& Burke, T. M. (June, 2011). The impact of race and SES on the credibility of an alibi. Paper presented at the Society for Applied Research on Memory and Cognition conference, New York, NY. 
Martin, R. (1992). The effects of ingroup-outgroup membership on minority influence when group membership is determined by a trivial categorization. Social Behavior and Personality, 20, 131-141. http://dx.doi.org/10.2224/sbp.1992.20.3.131

Masson, M. E. J., \& Loftus, G. R. (2003). Using confidence intervals for graphically based data interpretation. Canadian Journal of Experimental Psychology/Revue canadienne de psychologie expérimentale, 57, 203-220. doi: 10.1037/h0087426

McCloskey, J. (1989). Convicting the innocent. Criminal Justice Ethics, 8, 2-12. doi: 10.1080/0731129X.1988.9991845

Meertens, R. W., Koomen, W., Kelpeut, A. P., \& Hager, G. A. (1984). Effects of hypothesis and assigned task on question selection strategies. European Journal of Social Psychology, 14, 369-378. doi: 10.1002/ejsp.2420140403

Miller, J. G., Kahle, S., \& Hastings, P. D. (2015). Roots and benefits of costly giving: Children who are more altruistic have greater autonomic flexibility and less family wealth. Psychological Science, 26, 1038-1045. doi: 10.1177/0956797615578476

Mitchell, T. L., Haw, R. M., Pfeifer, J. E., \& Meissner, C. A. (2005). Racial bias in mock juror decision-making: A meta-analytic review of defendant treatment. Law and Human Behavior, 29, 621-637. http://dx.doi.org/10.1007/s10979-005-8122-9

Myers, B., Lynn, S. J., \& Arbuthnot, J. (2002). Victim impact testimony and juror judgments: The effects of harm information and witness demeanor. Journal of Applied Social Psychology, 32, 2393-2412. doi: 10.1111/j.1559-1816.2002.tb01869.x

Nakayachi, K. \& Kokima, S. (1988). The role of product schema in consumer decision making processes. Japanese Journal of Psychology, 59, 220-226.

National Registry of Exonerations (2015). Danny Brown - National registry of exonerations. Retrieved 2016, July 30 from http://www.law.umich.edu/special/exoneration/pages/casedetail.aspx?caseid=3059

National Registry of Exonerations (2013). Learn more. Retrieved 2013, Nov 12, from http://www.law.umich.edu/special/exoneration/Pages/learnmore.aspx

National Registry of Exonerations (2012, pre-June). Steven Avery. Retrieved from: https://www.law.umich.edu/special/exoneration/Pages/casedetail.aspx?caseid=3003

National Registry of Exonerations (n.d.). Exoneration case detail: Jose Garcia. Retrieved 2013, Oct 30 , from http://www.law.umich.edu/special/exoneration/Pages/casedetail.aspx?caseid=3835

Narby, D. J., Cutler, B. L., \& Moran, G. (1993). A meta-analysis of the association between authoritarianism and jurors' perceptions of defendant culpability. Journal of Applied 
Psychology, 78, 34-42. doi: 10.1037/0021-9010.78.1.34

Neisser, U., \& Harsch, N. (1992). Phantom flashbulbs: False recollections of hearing the news about Challenger. In E. Winograd, \& U. Neisser (Eds.) Affect and accuracy in recall: Studies of "flashbulb" memories (pp. 9-31). New York, NY: Cambridge University Press.

Nisbett, R. E., \& Wilson, T. D. (1977). Telling more than we can know: Verbal reports on mental processes. Psychological Review, 84, 231-259.

Northwestern Law (n.d.). Steven Avery. Retrieved from http://www.law.northwestern.edu/wrongfulconvictions/exonerations/wiAverySSummary. html

Office of the Correctional Investigator (2013, September 16). Backgrounder - Aboriginal offenders - A critical situation. Retrieved 2016, December 17 from: http://www.ocibec.gc.ca/cnt/rpt/oth-aut/oth-aut20121022info-eng.aspx

Ogloff, J. R. P., \& Rose, V. G. (2005). The comprehension of judicial instructions. In N. Brewer \& K. D. Williams (Eds.), Psychology and Law: An empirical perspective (pp. 407-444). New York, NY: The Guilford Press.

Olson, E. A. (2013). "You don't expect me to believe that, do you?" Expectations influence recall and belief of alibi information. Journal of Applied Social Psychology, 43, 12381247. doi: 10.1111/jasp. 12086

Olson, E. A. (2002). Where were you last night? Alibi believability and corroborating evidence: A new direction in psychology and law. Unpublished master's thesis, Iowa State University.

Olson, E. A., \& Charman, S. D. (2012). 'But can you prove it?' - Examining the quality of innocent suspects' alibis. Psychology, Crime \& Law, 18, 453-471. doi: 10.1080/1068316X.

Olson, E. A., \& Wells, G. L. (2012). The alibi-generation effect: Alibi generation experience influences alibi evaluation. Legal and Criminological Psychology, 17, 151-164. doi: 10.1111/j.2044-8333.2010.02003.x

Olson, E. A., \& Wells, G. L. (2004). What makes a good alibi? A proposed taxonomy. Law and Human Behavior, 28, 157-176. doi: 10.1023/B:LAHU0000022320.47112.d3

Olson, E. A. \& Wells, G. L. (2003, May). Alibi generation: Not so easy for the innocent. Poster session presented at the 15th annual meeting of the American Psychological Society, Atlanta, GA.

Overbeck, J. L. (2005). Beyond admissibility: A practical look at the use of eyewitness expert 
testimony in the federal courts. New York University Law Review, 80(6), 1895-1920.

Oxford English Dictionary (2013). Moral, adj. Retrieved 17 December, 2013 from http://www.oed.com/view/Entry/122086?rskey=b3tgKg\&result=2\&isAdvanced=false\#ei d

Palmer, M. A., Brewer, N., \& Weber, N. (2010). Postidentification feedback affects subsequent eyewitness identification performance. Journal of Experimental Psychology: Applied, 16, 387-398.

Paolacci, G., \& Chandler, J. (2014). Inside the turk: Understanding mechanical turk as a participant pool. Current Directions in Psychological Science, 23, 184-188. doi: $101177 / 0963721414531598$

Pfeifer, J. E., \& Olgoff, J. R. P. (1991). Ambiguity and guilt determinations: A modern racism perspective. Journal of Applied Social Psychology, 21, 1713-1725.

Pfeifer, J. E., \& Olgoff, J. R. P. (2003). Mock juror ratings of guilt in Canada: Modern racism and ethnic heritage. Social Behavior and Personality, 31, 301-312. http://dx.doi.org/10.2224/sbp.2003.31.3.301

Poulson, R. L. (1990). Mock juror attribution of criminal responsibility: Effects of race and the guilty byt mentally ill (GBMI) verdict option. Journal of Applied Social Psychology, 20, 1596-1611.

Preacher, K. J., \& Hayes, A. F. (2008). Asymptotic and resampling strategies for assessing and comparing indirect effects in multiple mediator models. Behavior Research Methods, 40, 879-891. http://dx.doi.org/10.3758/BRM.40.3.879

Price, R. \& Lovitt, J. T. (1997, February 12). Confusion for Simpson kids 'far from over'. Retrieved from: http://www.usatoday.com/news/index/nns224.htm

Poveda, T. G. (2001). Estimating wrongful convictions. Justice Quarterly, 18, 689-708. doi: $10.1080 / 07418820100095061$

Ramsey, R. J., \& Frank, J. (2007). Wrongful conviction: Perceptions of criminal justice professionals regarding the frequency of wrongful conviction and the extent of system errors. Crime \& Delinquency, 53, 436-470. doi: 10.1177/0011128706286554

Read, J. D. (1995). The availability heuristic in person identification: The sometimes misleading consequences of enhanced contextual information. Applied Cognitive Psychology, 9, 91121. doi: 10.1002/acp.2350090202

Read, J. D., \& Connelly, D. A. (2007). The effects of delay on long-term memory for witnessed events. In M. P. Toglia, J. D. Read, \& R. C. L. Lindsay (Eds.), The handbook of eyewitness psychology, Vol 1: Memory for events (pp. 117-155). Mahwah, NJ: Lawrench 
Erlhaum Associates Publishers.

Reeve, J. (2005). Understanding motivation and emotion (Fourth Edition). Hoboken, NJ: John Wiley \& Sons, Inc.

Reiss, J. (2015). Conviction. Harvard T.H. Chan school of public health. Retrieved 2015, December 18 from: http://www.hsph.harvard.edu/news/magazine/conviction/

Reynolds, K. (2010). The impact of alibi change on verdict decision. Unpublished master's thesis, Ryerson University.

Risen, J., \& Gilovich, T. (2007). Informal logical fallacies. In R. J. Sternberg, H. L. Roediger, \& D. F. Halpern (Eds.), Critical thinking in psychology (pp. 110-130). New York: Cambridge University Press.

Rozin, P., \& Royzman, E. B. (2001). Negativity bias, negativity dominance, and contagion. Personality and Social Psychology Review, 5, 296-320. doi: 10.1207/S15327957PSPR0504_2

$R v$ Demers (1926) 4 DLR 991 (Quebec)

$R v$ Gottschall 10 CCC 3d 447 (NSSC, 1983)

$R v$ Haynes 23 CCC101 (NSSC, 1914)

$R v$ Hibbert [2002] 2 SCR 445

R. v. Tessier (1997), 113 C.C.C. (3d) 538

Sargent, M. J., \& Bradfield, A. L., (2004). Race and information processing in criminal trials: Does the defendant's race affect how the facts are evaluated? Personality and Social Psychology Bulletin, 30, 995-1008. doi: 10.1177/0146167204265741

Scheck, B., Neufeld, P., \& Dwyer, J. (2000). Actual innocence: Five days to execution, and other dispatches from the wrongly convicted. New York: Random House.

Schmolk, H., Buffalo, E. A., \& Squire, L. R. (2000). Memory distortions develop over time: Recollections of the O.J. Simpson trial verdict after 15 and 32 months. Psychological Science, 11, 39-45.

Schram, J. (2013, 31 July). O.J. Simpson granted parole - not free yet as he still has to serve 4 more years of sentence. New York Post. Retrieved from: http://nypost.com/2013/07/31/oj-simpson-granted-parole-not-free-yet-as-he-still-has-to-serve-4-more-years-of-sentence/

Scroggs, J. R. (1976). Penalties for rate as a function of victim provocativeness, damage, and resistance. Journal of Applied Social Psychology, 4, 360-368. 
Semmler, C., Brewer, N., \& Douglass, A. B. (2012). Jurors believe eyewitnesses. In C.Semmler, N. Brewer, \& A. B. Douglass (Eds.), Conviction of the innocent: Lessonsfrom psychological research (pp. 185-209). Washington, DC, USA: American Psy-chological Association.

Sharma, V. (1988). Effect of model's amount of donation and justification on imitative altruism. Psycho-Lingua, 18, 39-46.

Sherrer, H. (2012). Has Steven Avery twice been wrongly convicted of heinous crimes? Jistice Denied: The magazine for the wrongly convicted, 52, 3-5. Retrieved 2013, November 21 from http://justicedenied.org/issue/issue_52/steven_avery_jd52.pdf

Skowronski, J. J., \& Carlston, D. E. (1987). Social judgment and social memory: The role of cue diagnosticity in negativity, positivity, and extremity biases. Journal of Personality and Social Psycholoty, 52, 689-699. http://dx.doi.org/10.1037/0022-3514.52.4.689

Smith, B., Zalman, M., \& Kiger, A. (2011). How justice system officials view wrongful convictions. Crime \& Delinquency, 57, 663-685. doi: 10.1177/0011128709335020

Snyder, M., \& Swann, W. B. (1978). Hypothesis-testing processes in social interaction. Journal of Personality and Social Psychology, 36, 1202-1212. doi: 10.1037/00223514.36.11.1202

Sommers, S.R. \& Douglass, A. B. (2007). Context matters: Alibi strength varies according to evaluator perspective. Legal and Criminological Psychology, 12, 41-54. doi: $10.1348 / 135532506 \times 114$

Sommers, S. R., \& Ellsworth, P. C. (2001). White juror bias: An investigation of prejudice against black defendants in the American courtroom. Psychology, Public Policy, and Law, 7, 201-229. http://dx.doi.org/10.1037/1076-8971.7.1.201

Sommers, S. R., \& Ellsworth, P. C. (2000). Race in the courtroom: Perceptions of guilt and dispositional attributions. Personality and Social Psychology Bulletin, 26, 1367-1379.

Sprecher, S. Effects of actual (manipulated) and perceived similarity of liking in get-acquainted interactions: The role of communication. Communication Monographs, 81, 4-27. http://dx.doi.org/10.1080/03637751.2013.839884

Statistics Canada (2011). National Household Survey [NHS] Profile, 2011. Retrieved from: http://www12.statcan.gc.ca/nhs-enm/2011/dp$\mathrm{pd} /$ prof/details/page.cfm?Lang $=\mathrm{E} \& \mathrm{Geo} 1=\mathrm{PR} \&$ Code $1=01 \&$ Data $=$ Count $\&$ SearchText $=\mathrm{Ca}$ nada\&SearchType $=$ Begins $\&$ SearchPR $=01 \& A 1=$ All $\& B 1=$ All $\&$ Custom $=\&$ TABID $=1$

Statistics Canada (2015, November 30). Aboriginal peoples in Canada: First Nations People, Métis and Inuit. Retrieved 2015 December 17 from: http://www12.statcan.gc.ca/nhs- 
enm/2011/as-sa/99-011-x/99-011-x2011001-eng.cfm

Strange, D., Dysart, J., \& Loftus, E. (2010). Where were you? Alibi generation, accuracy, and consistency. Paper presented at the American Psychology-Law Society conference, Vancouver, BC, Canada.

Strange, D., Dysart, J., \& Loftus, E. F. (2014). Why errors in alibis are not necessarily evidence of guilt. Zeitschrift für Psychologie, 222, 82-89. doi: 10.1027/2151-2604/a000169

Steblay, N. K., Tix, R. W., \& Benson, S. L. (2013). Double exposure: The effects of repeated identification lineups on eyewitness accuracy. Applied Cognitive Psychology, 27, 644654. doi: 10.1002/acp.2944

Sternglanz, R. W. (2004). Exoneration of serious wrongdoing via confession to a lesser crime (unpublished doctoral dissertation).University of Virginia, Charlottesville, VA.

Sunnafrank, M., \& Fontes, N. E. (1983). General and crime related racial stereotypes and influence on juridic decisions. Cornell Journal of Social Relations, 17, 1-15.

Sverdlik, N., Roccas, S., \& Sagiv, L. (2012). Morality across cultures: A values perspective. In M. Mikulincer \& P. R. Shaver (Eds.), The social psychology of morality: Exploring the causes of good and evil (pp. 219-235). Washington, DC: American Psychological Association.

Tabak, N., Bar-tal, Y., \& Cohen-Mansfield, J. (1996). Clinical decision making of experienced and novice nurses. Western Journal of Nursing Research, 18, 534-547. doi: $10.1177 / 019394599601800505$

Tajfel, H., Billig, M. G., Bundy, R. P., \& Flament, C. (1971). Social categorization and intergroup behaviour. European Journal of Social Psychology, 1, 149-178. http://dx.doi.org/10.1002/ejsp.2420010202

Taylor, D. M., \& Doria, J. R. (1981). Self-serving and group-serving bias in attribution. The Journal of Social Psychology, 113, 201-211. doi: 10.1080/00224545.1981.9924371

Taylor, S. E. \& Crocker, J. (1981). Schematic bases of social information processing. In E. T. Higgins, C. P. Herman, \& M. P. Zanna (Eds.), Social cognition: The Ontario symposium (Vol. 1, pp. 89-134). Hillsdale, NJ: Erlbaum.

The Forgiveness Project (2010). Penny Beerntsen (USA). Retrieved 2013, Nov 13 from http://theforgivenessproject.com/stories/penny-beernsten-usa/

The Innocence Project (n.d.a). The innocence project-Know the cases: Browse profiles: Steven Avery. Retrieved from http://www.innocenceproject.org/Content/Steven_Avery.php

The Innocence Project (n.d.b). The innocence project-Understand the causes: Eyewitness 
misidentification. Retrieved 2013, Oct 30, from

http://www.innocenceproject.org/understand/Eyewitness-Misidentification.php

The Innocence Project (n.d.c). The innocence project - know the cases: Browse Profiles:

Richard Alexander. Retrieved 2013, Oct 30, from

http://www.innocenceproject.org/Content/Richard_Alexander.php

The Innocence Project (n.d.d.). The innocence project - know the cases. Retrieved 18 December, 2013 from http://www.innocenceproject.org/know/

True Crime XL (2012). Steven Avery. Retrieved 2013, Nov 13 from http://truecrimecases.blogspot.ca/2012/08/steven-avery.html

Tuckey, M. R. \& Brewer, N. (2003). The influence of schemas, stimulus ambiguity, and interview schedule on eyewitness memory over time. Journal of Experimental Psychology: Applied, 9, 101-118. doi: 10.1037/1076-898X.9.2.101

Turner, J. C., Brown, R. J., \& Tajfel, H. (1979). Social comparison and group interest in ingroup favouritism. European Journal of Social Psychology, 9, 187-204. http://dx.doi.org/10.1002/ejsp.2420090207

United States v. Telfaire, 469 F.2d 552, 558-59 (D.C.Cir. 1972).

U.S. Census Bureau (2012). Section 1. Population. Retrieved from: http://www.census.gov/library/publications/2011/compendia/statab/131ed/population.htm 1

Walbert, K. (n.d.). American Indian vs. Native American: A note on terminology. Retrieved from: http://www.learnnc.org/lp/editions/nc-american-indians/5526

Wason, P. C. (1968). Reasoning about a rule. The Quarterly Journal of Experimental Psychology, 20, 273-281. doi: 10.1080/14640746808400161

Wicker, A. W. (1969). Attitudes versus actions: The relationship of verbal and overt behavioral responses to attitude objects. Journal of Social Issues, 25, 41-78.

Wilder, D. A., (1984). Predictions of belief homogeneity and similarity following social categorization. British Journal of Social Psychology, 23, 323-333. http://dx.doi.org/10.1111/j.2044-8309.1984.tb00648.xl

Williams, M. R., \& Holcomb, J. E. (2001). Racial disparity and death sentences in Ohio. Journal of Criminal Justice, 209, 207-218.

Wissler, R. L., Evans, D. L., Hart, A. J., Morry, M. M., \& Saks, M. J. (1997). Explaining "pain and suffering" awards: The role of injury characteristics and fault attributions. Law and Human Behavior, 21, 181-208. 
Wixted, J. T. \& Ebbesen, E. B. (1991). On the form of forgetting. Psychological Science, 2, 409415. doi: 10.1111/j.1467-9280.1991.tb00175.x

Wrightsman, L. S., Batson, A. L., \& Edkins, V. A. (Eds.). (2004). Measures of legal attitudes. Belmont, CA: Wadsworth.

Wyer, R. S., Srull, T. K., Gordon, S. E., \& Hartwick, J. (1982). Effects of processing objectives on the recall of prose material. Journal of Personality and Social Psychology, 43, 674688.

Zebrowitz, L. A., \& McDonald, S. M. (1991). The impact of litigants' baby-facedness and attractiveness on adjudications in small claims courts. Law and Human Behavior, 15, 603-623. doi: 10.1007/BF01065855

Zimmerman, C. A., \& Kelley, C. M. (2010). "I'll remember this!" Effects of emotionality on memory predictions versus memory performance. Journal of Memory and Language, 62, 240-253. doi: 10.1016/j.jml.2009.11.004 